\title{
Computational cell dosimetry for cancer radiotherapy and diagnostic radiology
}

by

Patricia A. K. Oliver

A thesis submitted to the Faculty of Graduate and Postdoctoral Affairs in partial fulfillment of the requirements for the degree of

Doctor of Philosophy

in

\begin{abstract}
Physics
Specialization in Medical Physics
\end{abstract}

Ottawa-Carleton Institute for Physics

Department of Physics

Carleton University

Ottawa, Ontario, Canada

2018

(c) 2018 Patricia A. K. Oliver 



\section{Abstract}

Computational radiation dosimetry, using e.g., Monte Carlo (MC) techniques, is used to calculate the amount of energy per unit mass deposited in tissue (the absorbed dose) resulting from radiotherapy and diagnostic radiology procedures. Motivated by the radiobiological importance of subcellular structures such as the nucleus, this work seeks to develop a better understanding of energy deposition on the cellular level.

Multicellular models of healthy and cancerous human soft tissues are developed based on typical values of cell compartment sizes, elemental compositions and number densities found in the literature. MC simulations are used to investigate how energy deposition within the nucleus and cytoplasm depends on incident photon energy, dose level, cell morphology, cell arrangement method, elemental composition, and the microscopic details of the environment surrounding the target of interest. MC simulations are carried out for monoenergetic kilovoltage incident photon energies ranging from 20 to $370 \mathrm{keV} .120 \mathrm{kVp}$ x-ray, Cobalt-60 and $6 \mathrm{MV}$ medical linac photon spectra are also considered. Given the considerable computational resources required for simulations involving microscopic details, cavity theory is explored as a method for relating macroscopic (bulk tissue) and microscopic (cellular) dose descriptors without the need for MC simulations.

For low doses and small target sizes, the stochastic nature of radiation transport and energy deposition can lead to considerable variation in energy deposition across a target population; this variation is referred to as the microdosimetric spread. The microdosimetric spread is investigated in the context of experimental radiation response studies using $\mu \mathrm{m}$-sized sampling volumes. These results are relevant for guiding measurement and data analysis techniques for Raman spectroscopy studies. Additionally, motivated by the lack of detail included in traditional breast dosimetry computational models, multiscale models of compressed breasts, combining macroscopic and micro- 
scopic features, are developed. A $30 \mathrm{kVp}$ mammography spectrum is considered.

The sensitivity of results with respect to microscopic tissue structure model parameters emphasizes the importance of accurate knowledge of cellular properties. For incident photon energies $<50 \mathrm{keV}$, there are considerable differences between macroscopic and microscopic dose descriptors. Results highlight the importance of microdosimetric considerations when scoring energy deposition in subcellular targets. 


\section{Acknowledgments}

I would like to thank Dr. Rowan Thomson for being an excellent supervisor and mentor who is helpful, encouraging and inspirational. I would also like to thank Dr. Dave Rogers for helpful comments and suggestions related to cavity theory, and for being a wonderful teacher. Members of the Carleton Laboratory for Radiotherapy Physics (CLRP) are thanked for their support: Martin Martinov, Dr. Nelson Miksys, Stephen Deering, Mehan Haidari, Victor Malkov, and Zack Parsons.

I am very grateful to my parents for their unconditional support, encouragement and love. They have made many sacrifices and have been unbelievably patient. I would like to thank Connor for being a wonderful and loving partner who always knows how to make me laugh.

Martin Martinov is thanked for providing the modified version of the EGSnrc usercode egs_chamber that is capable of scoring energy deposition in multiple regions. Dr. S. Murugkar, Dr. V. Chauhan, Dr. B. Nyiri, H. Allen and Dr. A. Jirasek are thanked for helpful conversations with the authors related to chapter 6. James Sawicki is thanked for answering questions related to the segmented breast phantoms obtained from the University of Wisconsin Numerical Breast Phantom Repository used in chapter 7.

Carleton University, the Natural Sciences and Engineering Research Council of Canada (NSERC), the Ontario Graduate Scholarship, the O'Brien Foundation Alumni Fellowship, the Kiwanis Club of Ottawa Medical Foundation and Dr. Kanta Marwah Scholarship are acknowledged as sources of financial assistance. I would also like to thank Compute/Calcul Canada and the Shared Hierarchical Academic Research Computing Network (SHARCNET) for access to computing resources.

Finally, I would like to express my appreciation to my Ph.D. committee for their time and effort: Dr. Rowan Thomson, Dr. Sangeeta Murugkar and Dr. Karin Hinzer. 


\section{Statement of originality}

This thesis is a record of the author's research as a graduate student at Carleton University. Much of the content in this document has been published in peer-reviewed journals, and/or presented at scientific conferences.

My supervisor Dr. Rowan Thomson has contributed to all aspects of the research summarized herein. She has guided my research, provided suggestions, and edited all publications and conference presentations before they were finalized. Aside from the assistance provided by Dr. Thomson, all computational work, data analysis, writing, and manuscript revision prior to publication was carried out by the author of this thesis.

\section{Peer-reviewed papers}

1. P. A. K. Oliver and R. M. Thomson, "A Monte Carlo study of macroscopic and microscopic dose descriptors for kilovoltage cellular dosimetry," Phys. Med. Biol. 62 (4), 1417 - 1437 (2017). — see chapter 3

2. P. A. K. Oliver and R. M. Thomson, "Cavity theory applications for kilovoltage cellular dosimetry," Phys. Med. Biol. 62 (11), 4440 - 4459 (2017). — see chapter 4

3. P. A. K. Oliver and R. M. Thomson, "Multiscale Monte Carlo simulations investigating energy deposition within cell populations," accepted for publication in Phys. Med. Biol. on April 3, 2018. - see chapter 5

4. P. A. K. Oliver and R. M. Thomson, "Microdosimetric considerations for radiation response studies using Raman spectroscopy," accepted for publication in Med. Phys. on April 12, 2018. — see chapter 6

5. P. A. K. Oliver and R. M. Thomson, "Investigating energy deposition in glandular tissues for mammography using multiscale Monte Carlo simulations," manuscript in preparation. - see chapter 7 


\section{Conference abstracts}

(* indicates presenting author)

- World Congress on Medical Physics and Biomedical Engineering, Toronto, ON, 2015: *P. Oliver and R. Thomson, "Investigation of the relations between absorbed dose to cellular targets and to bulk tissue for kilovoltage radiation using Monte Carlo simulations and cavity theory" (oral presentation)

- AAPM annual meeting and exhibition, Anaheim, CA, 2015: *P. Oliver and R. Thomson, "A Monte Carlo study of macroscopic and microscopic dose descriptors for kilovoltage cellular dosimetry," Med. Phys. 42 (6), 3674 (2015). (oral presentation)

- COMP annual scientific meeting, St. Johns, NL, 2016: *P. Oliver and R. Thomson, "A Monte Carlo study of macro- and microscopic dose descriptors and the microdosimetric spread using detailed cellular models," Med. Phys. 43 (8), 4929 (2016). (oral presentation at Young Investigator's Symposium)

- COMP annual scientific meeting, Ottawa, ON, 2017: *P. Oliver and R. Thomson, "Investigating energy deposition in glandular tissues for mammography using multiscale Monte Carlo simulations," Med. Phys. 44 (8), 4370 (2017). (oral presentation at Young Investigator's Symposium)

- AAPM annual meeting and exhibition, Denver, CO, 2017: *P. Oliver and R. Thomson, "Cavity theory applications for kilovoltage cellular dosimetry," Med. Phys. 44 (6), 2745 (2017). (oral presentation)

- International Conference on Monte Carlo Techniques for Medical Applications, Napoli, Italy, 2017: *P. Oliver and R. Thomson, "Investigating energy deposition in cellular targets using multiscale tissue models," Physica Medica 42, 7 (2017). (poster presentation) 


\section{List of abbreviations and symbols}

AdCC $\ldots . . .$. adenoidcystic carcinoma

CPE $\ldots \ldots \ldots$ charged particle equilibrium

CSE $\ldots \ldots \ldots$ c.... cross section enhancement

$\boldsymbol{D}_{\text {cyt }} \quad \ldots \ldots \ldots$ dose to cytoplasm in a multicellular model

$D_{\text {cyt }}^{1} \quad \ldots \ldots \ldots$ dose to cytoplasm in a geometry consisting of a single cell

$\boldsymbol{D}_{\boldsymbol{g l}, \boldsymbol{R O I}} \ldots \ldots \ldots$ glandular voxel dose

$\boldsymbol{D}_{\boldsymbol{m}, \boldsymbol{m}} \ldots \ldots \ldots$ dose to a cavity of medium $m$ embedded in medium $m$;

dose-to-medium-in-medium*

$\boldsymbol{D}_{m, m}^{c} \ldots \ldots \ldots$. dose to the cytoplasm of cell of medium $m$ embedded in medium $m$

$\boldsymbol{D}_{n u c} \ldots \ldots \ldots$ dose to nucleus in a multicellular model

$D_{n u c}^{1} \ldots \ldots \ldots$ dose to nucleus in a geometry consisting of a single cell

$\boldsymbol{D}_{n u c, m} \ldots \ldots$. dose to a nucleus embedded in medium $m^{*}$

$\boldsymbol{D}_{\boldsymbol{w}, \boldsymbol{m}} \ldots \ldots \ldots$ dose to a water cavity embedded in medium $m$; dose-to-water-inmedium*

$\boldsymbol{D}_{\boldsymbol{w}, m}^{c} \ldots \ldots \ldots$ dose to the cytoplasm of a single water cell embedded in medium $m$

ECM $\ldots \ldots \ldots$ extracellular matrix

ICT $\ldots \ldots \ldots \ldots$ intermediate cavity theory

LCT $\ldots \ldots \ldots$ large cavity theory

MBDCA $\ldots . . \quad$ model-based dose calculation algorithm

MC $\ldots . . . . . . . \quad$ Monte Carlo

MCL ….... mean chord length

MGD ........ mean glandular dose 
$\boldsymbol{r}_{\text {cell }} \ldots \ldots \ldots$ cell radius

$\boldsymbol{R}_{\boldsymbol{C S D A}} \ldots \ldots$ range according to the continuous slowing down approximation

$\boldsymbol{r}_{\text {nuc }} \ldots \ldots \ldots$ nuclear radius

RS ......... Raman spectroscopy

$\boldsymbol{S}_{\text {col }} / \boldsymbol{\rho} \ldots \ldots$ mass collisional stopping power

SCT $\ldots \ldots \ldots$ small cavity theory

SqCLC ..... squamous cell lung carcinoma

$\boldsymbol{z} \ldots \ldots \ldots \ldots$ energy imparted per unit mass; specific energy

$\bar{z} \ldots \ldots \ldots \ldots$ mean specific energy

$\boldsymbol{\mu}_{\boldsymbol{e n}} / \rho \ldots \ldots \ldots$ mass energy absorption coefficient

$\sigma_{z} \ldots \ldots \ldots$ standard deviation of the specific energy

$\sigma_{z} / \bar{z} \ldots \ldots \ldots$ relative standard deviation of the specific energy (quantifies the microdosimetric spread)

* In general, $D_{i, j}$ is the dose to a cavity containing medium $i$ embedded in medium $j$. 


\section{Contents}

1 Introduction $\quad 1$

1.1 Radiation use in medicine . . . . . . . . . . . . . . . . 2

1.2 Quantifying energy deposition .................. 4

1.3 Radiation dosimetry on the cellular level . . . . . . . . . . . . . 8

1.4 Linking macro- and microscopic dose descriptors . . . . . . . . . . . . . 9

1.5 Current status of cell dosimetry research . . . . . . . . . . . . . 11

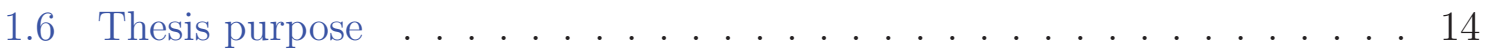

1.7 Thesis outline . . . . . . . . . . . . . . . . . . 15

2 Methods $\quad 16$

2.1 Monte Carlo simulations using the EGSnrc software . . . . . . . . . 17

2.2 Media definitions . . . . . . . . . . . . . . . . . . 19

2.3 Literature survey of cell and nucleus sizes . . . . . . . . . . . . . 24

3 Monte Carlo study of macro- and microscopic dose descriptors for $\begin{array}{ll}\text { kilovoltage cellular dosimetry } & 26\end{array}$

3.1 Introduction . . . . . . . . . . . . . . . . . 27

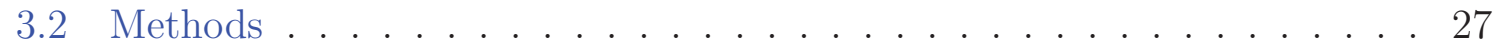

3.2.1 Development of microscopic tissue structure models . . . . . . . . 27

3.2.2 Monte Carlo simulations . . . . . . . . . . . . . . . . . . 28 
3.3 Results . . . . . . . . . . . . . . . . . . . . . . . . . . . . . 29

3.3.1 Surrounding geometry effects on nuclear and cytoplasm doses . . 29

3.3.2 Nuclear and cytoplasm dose variations with cell size . . . . . . . . 34

3.3.3 Comparison of macroscopic and microscopic dose descriptors . . . 37

3.4 Discussion . . . . . . . . . . . . . . . . . . . . . . . . . . 39

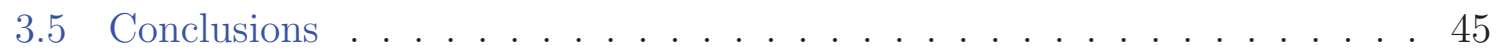

4 Cavity theory applications for kilovoltage cellular dosimetry $\quad 47$

4.1 Introduction . . . . . . . . . . . . . . . . 48

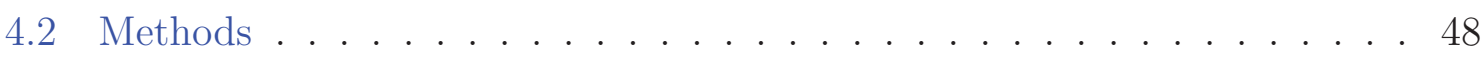

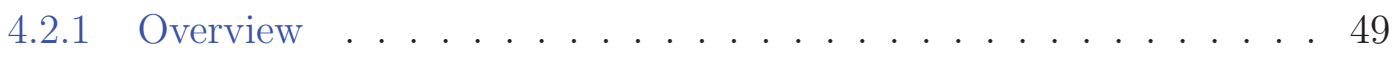

4.2.2 Monte Carlo simulations . . . . . . . . . . . . . . 52

4.2.3 Large, small and intermediate cavity theories . . . . . . . . . . 52

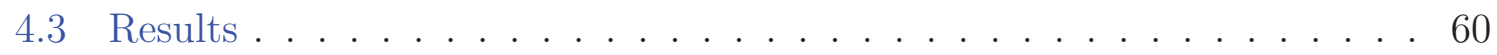

4.3.1 Evaluation of cavity theory approaches (part I) . . . . . . . . 60

4.3.2 Cavity theory applied to nuclear doses (part II) . . . . . . . . 63

4.3.3 Using Bragg-Gray small cavity theory to estimate dose to small water cavities (part III) . . . . . . . . . . . . . . 68

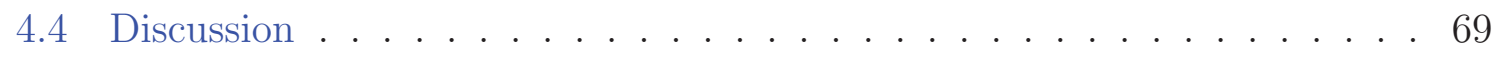

4.4.1 Evaluation of cavity theory approaches (part I) . . . . . . . . 69

4.4.2 Cavity theory applied to nuclear doses (part II) . . . . . . . . 70

4.4.3 Using Bragg-Gray small cavity theory to estimate dose to small water cavities (part III) . . . . . . . . . . . . 72

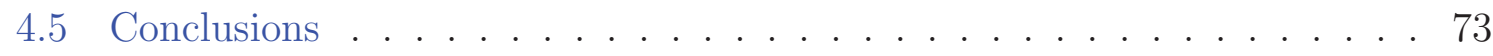

5 Multiscale Monte Carlo simulations investigating energy deposition $\begin{array}{ll}\text { within cell populations } & 75\end{array}$ 
5.1 Introduction . . . . . . . . . . . . . . . . 76

5.2 Methods . . . . . . . . . . . . . . . . . . . . 76

5.2.1 Development of microscopic tissue structure models . . . . . . . . 76

5.2.2 Monte Carlo simulations and analysis of results . . . . . . . 80

5.3 Results. . . . . . . . . . . . . . . . . . . . . 83

5.4 Discussion . . . . . . . . . . . . . . . . . . . . . . . . . . . . 91

5.5 Conclusions . . . . . . . . . . . . . . . . . . . . . 96

6 Microdosimetric considerations for radiation response studies using $\begin{array}{ll}\text { Raman spectroscopy } & 97\end{array}$

6.1 Introduction . . . . . . . . . . . . . . . . . . . . . 98

6.2 Methods . . . . . . . . . . . . . . . . . . . . . . . 100

6.3 Results . . . . . . . . . . . . . . . . . . . . . . . . . . 103

6.3.1 Part 1: multicellular models . . . . . . . . . . . . . . . 103

6.3.2 Part 2: cubic voxel geometries . . . . . . . . . . . . . 104

6.4 Discussion . . . . . . . . . . . . . . . . . . . . . . . 110

6.5 Conclusions . . . . . . . . . . . . . . . . . . . . . . . . 113

7 Investigating energy deposition in glandular tissues for mammography $\begin{array}{ll}\text { using multiscale Monte Carlo simulations } & 114\end{array}$

7.1 Introduction . . . . . . . . . . . . . . . . . . . . 115

7.2 Methods . . . . . . . . . . . . . . . . . . . . 116

7.2.1 Development of multiscale models of compressed breasts . . . . 116

7.2.2 Monte Carlo simulations . . . . . . . . . . . . . . . . 120

7.3 Results . . . . . . . . . . . . . . . . . . . . . . . . . . 122

7.3.1 Macroscopic dose distributions in segmented breast tissue phantoms122

7.3.2 Specific energy distributions within cell populations . . . . . . . 125 
7.3.3 Sensitivity analysis for specific energy distributions . . . . . . . . 128

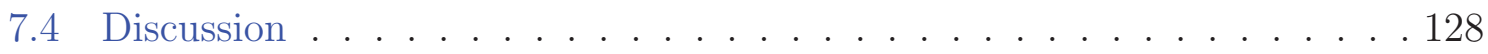

7.5 Conclusions . . . . . . . . . . . . . . . . . . . . . . 134

8 Conclusion 135

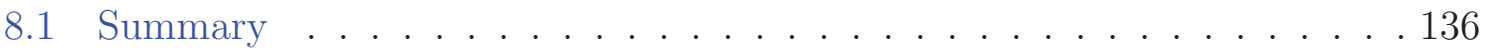

8.2 Outlook and ideas for future work . . . . . . . . . . . . . 139

Appendices

143

A Transport parameter tests

143

B Supplementary material for chapter 5

146

References $\quad 165$ 


\section{List of Tables}

2.1 Elemental compositions, water content and mass densities of media used in $\mathrm{MC}$ simulations . . . . . . . . . . . . . . . . . . . 23

2.2 Literature survey of human cell and nucleus sizes . . . . . . . . . . . 25

3.1 Microscopic tissue structure model parameters for 13-cell clusters . . . . 28

4.1 Summary of intermediate cavity theory approaches . . . . . . . . . 58

4.2 Values of intermediate cavity theory parameter $d$ according to the 'BurlinJanssens' method . . . . . . . . . . . . . . . . . . 65

5.1 Nominal and actual mean radii, and actual mean volumes of subcellular compartments for populations of $>1500$ cells . . . . . . . . . . . . 79

5.2 Fit parameters for the equation relating $\sigma_{z} / \bar{z}$ to $D_{m, m}$ and the target volume for multicellular models . . . . . . . . . . . . . . . . . . . 90

5.3 Example results for various microdosimetric quantities for multicellular

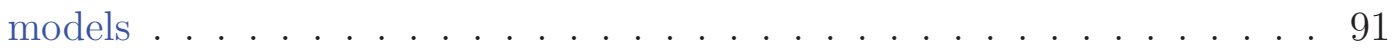

6.1 Fit parameters for the equation relating $\sigma_{z} / \bar{z}$ to the target volume and dose for cubic voxel geometries . . . . . . . . . . . . . . . . 107

6.2 Select results for various microdosimetric quantities for cubic voxel ge-

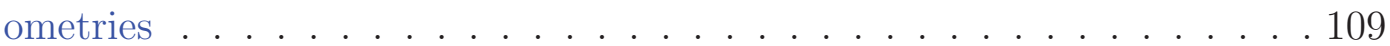

B.1 Summary of microdosimetric results for $20 \mathrm{keV}$, melanoma for cubic voxel geometries . . . . . . . . . . . . . . . . . . 147 
B.2 Summary of microdosimetric results for $30 \mathrm{keV}$, melanoma for cubic voxel

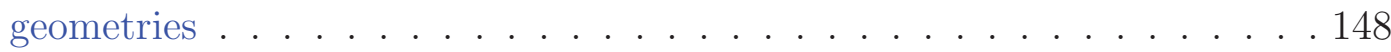

B.3 Summary of microdosimetric results for $50 \mathrm{keV}$, melanoma for cubic voxel geometries . . . . . . . . . . . . . . . . . . . . 149

B.4 Summary of microdosimetric results for $90 \mathrm{keV}$, melanoma for cubic voxel geometries . . . . . . . . . . . . . . . . . . 150

B.5 Summary of microdosimetric results for $370 \mathrm{keV}$, melanoma for cubic voxel geometries . . . . . . . . . . . . . . . . . . . . . . 151

B.6 Summary of microdosimetric results for ${ }^{60} \mathrm{Co}$, melanoma for cubic voxel

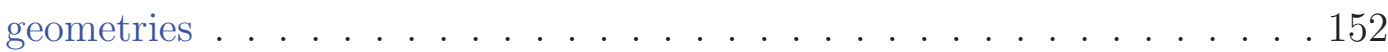

B.7 Summary of microdosimetric results for $20 \mathrm{keV}$, muscle for cubic voxel geometries . . . . . . . . . . . . . . . . . . 153

B.8 Summary of microdosimetric results for $30 \mathrm{keV}$, muscle for cubic voxel

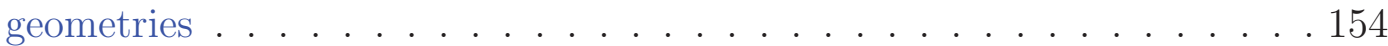

B.9 Summary of microdosimetric results for $50 \mathrm{keV}$, muscle for cubic voxel geometries . . . . . . . . . . . . . . . . . 155

B.10 Summary of microdosimetric results for $90 \mathrm{keV}$, muscle for cubic voxel

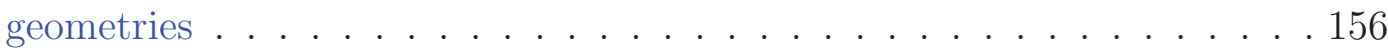

B.11 Summary of microdosimetric results for $370 \mathrm{keV}$, muscle for cubic voxel geometries . . . . . . . . . . . . . . . . . . . 157

B.12 Summary of microdosimetric results for ${ }^{60} \mathrm{Co}$, muscle for cubic voxel geometries . . . . . . . . . . . . . . . . . . . . . . . 158

B.13 Summary of microdosimetric results for $20 \mathrm{keV}$, mammary gland for cubic voxel geometries . . . . . . . . . . . . . . . . . . . . 159

B.14 Summary of microdosimetric results for $30 \mathrm{keV}$, mammary gland for cubic voxel geometries . . . . . . . . . . . . . . . . . . . . . 160

B.15 Summary of microdosimetric results for $50 \mathrm{keV}$, mammary gland for cubic voxel geometries . . . . . . . . . . . . . . . . . . 161 
B.16 Summary of microdosimetric results for $90 \mathrm{keV}$, mammary gland for cubic voxel geometries . . . . . . . . . . . . . . . . . . . 162

B.17 Summary of microdosimetric results for $370 \mathrm{keV}$, mammary gland for cubic voxel geometries . . . . . . . . . . . . . . . . . . 163

B.18 Summary of microdosimetric results for ${ }^{60} \mathrm{Co}$, mammary gland for cubic voxel geometries . . . . . . . . . . . . . . . . . . . 164 


\section{List of Figures}

$2.1 \mu_{e n} / \rho$ and $S_{c o l} / \rho$ ratios with respect to water for cellular media . . . . . 21

$2.2 \mu_{e n} / \rho$ and $S_{c o l} / \rho$ ratios with respect to water for original and modified bulk tissues . . . . . . . . . . . . . . . . . . . . 22

3.1 Diagram of microscopic tissue structure models involving 13-cell clusters 30

3.2 Ratios of cellular dose descriptors in different surrounding environments . 31

3.3 Ratios of nuclear doses with different cell/nucleus sizes . . . . . . . . 35

3.4 Ratios of cytoplasm doses with different cell/nucleus sizes . . . . . . . . 36

3.5 Ratios of micro- and macroscopic dose descriptors for $r_{\text {cell }}=7.35 \mu \mathrm{m} \quad$. 38

3.6 Ratios of nuclear and macroscopic dose descriptors . . . . . . . . . . 40

3.7 Ratios of cytoplasm and macroscopic dose descriptors . . . . . . . . . . 41

4.1 Schematic diagrams summarizing the cavity theory study . . . . . . . . 50

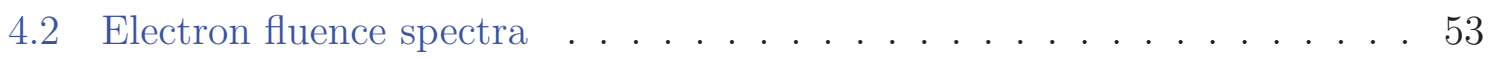

4.3 Spectrum averaged $\mu_{e n} / \rho$ and $S_{\text {col }} / \rho$ ratios . . . . . . . . . . 55

4.4 Intermediate cavity theory parameter $d$ as a function of energy . . . . . . 61

4.5 $D_{w, m} / D_{m, m}$ according to Monte Carlo and various cavity theory methods 62

4.6 Histograms of differences between cavity theory estimates and Monte

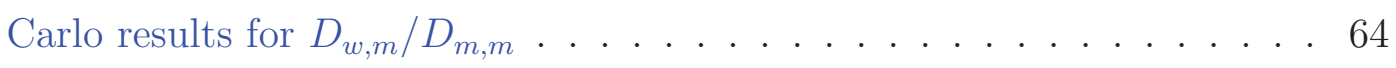

4.7 'Burlin-Janssens' intermediate cavity theory predictions and Monte Carlo results for dose-to-nucleus $/ D_{m, m}$, for $r_{\text {cell }}=7.35 \mu \mathrm{m} \ldots$. . . . . . . 66 
4.8 Ratios of 'Burlin-Janssens' intermediate cavity theory predictions and Monte Carlo results for dose-to-nucleus $/ D_{m, m} \ldots \ldots$. . . . . . . . 67

4.9 Ratios of dose estimates for small volumes of water according to small cavity theory . . . . . . . . . . . . . . . . . 68

5.1 Cross sections of microscopic tissue structure models with $>1500$ cells $\quad 77$

5.2 Distributions of radii for two example cell and nucleus sizes for populations of $>1500$ cells . . . . . . . . . . . . . . . . . . . . . 79

5.3 Specific energy distributions for nuclear targets for different cell/nucleus sizes and energies . . . . . . . . . . . . . . . . . . . . 84

5.4 Fraction of nuclei receiving no energy deposition as a function of dose . . 86

5.5 Specific energy distributions for nuclear targets compared with corresponding normal distributions . . . . . . . . . . . . . . . . . 87

5.6 Ratios of $\bar{z}$ for nuclear and cytoplasm targets and $D_{m, m}$ as a function of incident photon energy . . . . . . . . . . . . . . . 89

5.7 Relative standard deviation of the specific energy for nuclear and cytoplasm targets as a function of $D_{m, m}$ for $\left(r_{\text {cell }}, r_{n u c}\right)=(7.5,6) \mu \mathrm{m} \ldots . . .90$

6.1 Schematic diagrams of MC simulation geometries relevant for Raman spectroscopy cellular radiation response studies . . . . . . . . . . . . 101

6.2 Specific energy distributions demonstrating sensitivity to Raman spectroscopy target type . . . . . . . . . . . . . . . . 105

6.3 Relative standard deviation of the specific energy for cubic voxel geometries as a function of dose . . . . . . . . . . . . . . . 106

6.4 Specific energy distributions for cubic voxels and corresponding normal distributions . . . . . . . . . . . . . . . . . . . 108

7.1 Multiscale mammography model with compressed breast . . . . . . . . 118

7.2 Microscopic tissue structure models for multiscale compressed breast models . . . . . . . . . . . . . . . . . . . . . 119 
7.3 Segmented breast tissue structure and corresponding dose distributions at various depths for phantom $012204 \ldots \ldots$. . . . . . . . . 123

7.4 Segmented breast tissue structure and corresponding dose distributions for phantoms 012804 and 062204 . . . . . . . . . . . . . . 124

7.5 Specific energy distributions for mammary epithelial cell nuclei and glandular tissue dose descriptors for different dose levels and ROIs . . . . . . 126

7.6 Relative standard deviation of the specific energy for mammary epithelial cell nuclei as a function of $D_{g l, R O I} \ldots \ldots \ldots \ldots \ldots \ldots \ldots \ldots$

7.7 Specific energy distributions for mammary epithelial cell nuclei demonstrating sensitivity to various model parameters . . . . . . . . . . . 129

A.1 Transport and variance reduction parameter sensitivity test results . . . . 144 


\section{Chapter 1}

\section{Introduction}

This Chapter introduces the use of ionizing radiation in medical applications including treatment and diagnostic contexts. An overview of radiation dosimetry concepts is provided. The Monte Carlo technique is introduced as a method for calculating energy deposition in arbitrary, user-defined simulation geometries. The importance of considering different lengths scales, from macroscopic bulk tissue structure to microscopic (i.e., cellular) targets is discussed. Past research and recent advances in radiation dosimetry are summarized, with particular focus on cellular dosimetry and computational models of microscopic tissue structure; limitations of these past studies are discussed. The stochastic nature of radiation transport and energy deposition, and its relevance to cellular dosimetry are outlined. 


\subsection{Radiation use in medicine}

Ionizing radiation is used in medicine for both diagnosis and treatment. Radiation therapy (or 'radiotherapy') is a cancer treatment that uses ionizing radiation to kill malignant cells (e.g., the tumour) while minimizing the damage to healthy cells (e.g., organs at risk). ${ }^{1}$ Different radiotherapy treatment modalities exist including a variety of delivery methods (e.g., external beam radiotherapy, brachytherapy), particle types (e.g., photons, electrons), and energies (from $\mathrm{keV}$ to $\mathrm{MeV}$ ), with corresponding differences in penetration depth and energy deposition characteristics. External beam radiotherapy (EBRT) has been historically delivered with a Cobalt-60 source (mean photon energy $1.25 \mathrm{MeV})$. Currently, photon and electron beams generated in linear accelerators are more common. EBRT delivery has progressed from relatively simple treatment plans involving a few hand-selected gantry angles (e.g., three-dimensional conformal radiation therapy, 3DCRT) toward intensity modulated radiation therapy (IMRT), which can feature step-and-shoot or continuous gantry rotation, continuous multileaf collimator (MLC) motion for beam aperture modulation, and dose rate modulation..$^{2,3}$ Image-guided radiotherapy (e.g., radiosurgery with respiratory motion compensation technology ${ }^{4}$ ) allows for further optimization of beam conformality.

The increased freedom in treatment delivery parameters afforded by IMRT and corresponding treatment planning algorithms yields highly conformal treatment plans, which allow for safe dose escalation to the target. ${ }^{2}$ However, having radiation incident from all angles around the patient results in a large region of normal patient anatomy receiving 'out-of-field' radiation. Kry et al. ${ }^{5}$ report that the risk of fatal secondary cancer increases from $1.7 \%$ for a 18 MV 3DCRT prostate cancer procedure (constant intensity across each irradiation field) to $5.1 \%$ for the corresponding $18 \mathrm{MV}$ IMRT procedure (where each irradiation field is divided up into subfields with differing intensities). In 
addition to secondary cancer risk, tumour control and short-term normal tissue reactions must also be considered when selecting the best treatment option. In addition to localized irradiation at specific disease sites, total body irradiation (TBI) is used in the treatment of e.g., leukemia, where it serves a dual purpose: killing malignant cells and immune suppression for the prevention of bone marrow transplant rejection. ${ }^{6}$ TBI results in considerable out-of-field radiation. Instead of irradiating the body as a whole, helical tomotherapy can be used to target bone marrow specifically. Helical tomotherapy delivers radiation in a slice-by-slice fashion, similar to the way that a computed tomography (CT) scan is acquired. ${ }^{7}$

In contrast with EBRT, brachytherapy involves the internal application of radioactive sources (implantation near or in malignant tissue), and is commonly used for prostate and gynaecological cancers. These radioactive sources can be permanent implants (with low dose rates), or they can be inserted into the patient for a short period of time using a remote afterloading unit, usually featuring high dose rate sources facilitating shorter treatment times. ${ }^{8}$

Diagnostic radiology involves the use of ionizing radiation to obtain an image of patient anatomy for the purposes of disease screening and diagnosis. From simple x-ray imaging with a single projection, to real time imaging using fluoroscopy, to CT scans producing three dimensional image of patient anatomy, the goal of diagnostic radiology is to yield information (e.g., an image) that a physician can use for diagnosis (or prognosis). ${ }^{9}$ Given that exposure to radiation can lead to DNA damage, possibly resulting in cancer induction, ${ }^{10}$ the benefits of diagnostic radiology (i.e., lives saved by diagnosis and subsequent treatment) must outweigh the risks. ${ }^{11}$ As an example, mammography is an x-ray imaging technique that is used to screen for and diagnose breast cancer. In Ontario, the recommended screening protocol consists of biennial exams for women between the ages of 50 and $74 .{ }^{12}$ A typical exam consists of a craniocaudal and a mediolateral oblique image of each breast, for a total of four images, with the breast 
compressed between two plates in each case. ${ }^{13}$

\subsection{Quantifying energy deposition}

The field of medical physics spans a wide range of goals, from advancing knowledge of radiation transport and energy deposition within a variety of scenarios and on various length scales, to clinical applications that introduce new techniques and improve upon existing treatments. Quantifying energy deposition resulting from radiation exposure (i.e., radiation dosimetry) is important for all uses of radiation in medicine. In radiotherapy, accurate dosimetry is necessary for ensuring that malignant cells are killed while normal tissue damage is minimized. Irradiation of especially radiosensitive tissues can be minimized by appropriate choice of beam angle, or by increasing the magnitude of the corresponding penalty to the cost function that the inverse treatment planning algorithm seeks to minimize. ${ }^{14}$ In diagnostic radiology, the goal is to minimize patient exposure, while ensuring image quality is adequate for diagnosis. The absorbed dose is relevant for macroscopic-scale energy deposition, and is defined as the mean energy imparted by ionizing radiation to matter per unit mass; units: $\mathrm{J} / \mathrm{kg}=\mathrm{Gy}$.

The absorbed dose, $D$, is a deterministic quantity; its stochastic analogue is the specific energy, $z$, which is defined as the energy imparted $\epsilon$ per unit mass $m$. Furthermore, $D=d \bar{\epsilon} / d m=\bar{z}$, where $d \bar{\epsilon}$ is the mean energy imparted to an infinitesimal volume of mass $d m$, and $\bar{z}$ is the mean specific energy. ${ }^{15}$ The specific energy distribution $f(z, D)$ is a probability density function for a target receiving specific energy $z$; $f(z, D)$ depends on the corresponding absorbed dose $D$ to a macroscopic region that

encompasses the targets, and is normalized such that $\int_{0}^{\infty} f(z, D) d z=1$. For high doses and large target sizes, $f(z, D)$ is normal, with a relatively small standard deviation so that the macroscopic dose is approximately the same as the dose to any small region encompassed within it. In contrast, for low doses and small target sizes, the stochastic nature of radiation transport and energy deposition is an important consideration, 
potentially resulting in considerable variation in energy deposition across a population of cells: ${ }^{16}$ this variation is referred to as the microdosimetric spread. The standard deviation of specific energy relative to the mean $\left(\sigma_{z} / \bar{z}\right)$ quantifies the microdosimetric spread, which generally decreases with increasing dose and target size. ${ }^{17}$

In radiotherapy, unanticipated variation in energy deposition may lead to lack of tumour cell eradication, and/or increased incidence of normal tissue complications. ${ }^{18,19}$ In diagnostic radiology, the risk of secondary cancer induction is predicted to vary (increasing in general) depending on dose level. ${ }^{11}$ Extrapolating to microscopic scales, it is expected that variation in energy deposition will lead to corresponding variation in cellular radiation responses (e.g., damage to subcellular components, ${ }^{20}$ production of molecules related to radiation-induced damage repair and signalling $\left.{ }^{21}\right)$. Radiation response is also determined by biological effects such as variation in radiosensitivity with cell cycle stage, ${ }^{10}$ and bystander effects ${ }^{22}$ ). Furthermore, uncertainty in the experimental setup (e.g., the radiation source, and measurement equipment) can also contribute to variation in measured radiation response.

Different methods exist for determination or estimation of absorbed dose and specific energy, depending on context. Macroscopic measurements of absorbed dose are commonly obtained using ionization chambers, which feature a gas filled sensitive region surrounded by an outer electrode (the wall) and containing a central charge collection electrode. Ionization chamber irradiation results in ionization of air within the sensitive region. Electrometers are used in conjunction with ionization chambers, allowing for measurement of the charge collected by the central electrode. ${ }^{23}$ Silicon p-n junction diodes are useful for in vivo dosimetry. Diode irradiation results in the production of electron-hole pairs throughout the device. Electron-hole pairs produced within or near (within one diffusion length) the depletion zone are separated, with the holes (electrons) being pulled toward the p-side (n-side) due to the electric field of the depletion region, generating a measurable current in the reverse direction. ${ }^{8}$ Radiochromic film can be 
used to obtain a visual representation of the dose distribution; polymerization of the dye within the film changes the light transmission properties. ${ }^{24}$ The quantity that is measured directly in each of the three examples above (i.e., charge/current from central electrode, diode current, and optical density) can be converted to absorbed dose via conversion factors or calibration information.

The dosimetry systems described above are useful for measurements on the macroscopic scale (down to $\sim$ mm length scales). Traditional experimental microdosimetry studies concerned with measuring energy deposition on $\sim \mu \mathrm{m}$ length scales typically use tissue equivalent proportional counters, consisting of a low density sphere (see e.g., Zellmer et $\left.a .^{25}\right)$. Energy deposition within this sphere is assumed to be equivalent to that of a smaller, higher density sphere such as the nucleus, thereby avoiding the need to manufacture a $\mu \mathrm{m}$-sized detector. ${ }^{17}$ A better understanding of radiobiological effects (e.g., with respect to the role played by various organelles) can be achieved with microbeam irradiation, which can be used to aim single particles toward subcellular targets with $\sim \mu \mathrm{m}$ accuracy, thus eliminating the stochastic uncertainty associated with whether or not a target has been hit in conventional broad beam irradiation. ${ }^{26}$ Fluorescent nuclear track detectors (FNTDs) coated with cells are another method for investigating energy deposition on the microscopic scale which eliminates the stochastic uncertainty associated with radiation transport: FNTD crystal defects trap secondary electrons, resulting in fluorescence during the readout process involving confocal laser scanning microscopy; this technique allows particle tracks to be reconstructed, which can be correlated with the corresponding radiation-induced damage to the cells that coat the crystal. ${ }^{27}$ The development of computational techniques such as the Monte Carlo (MC) approach has allowed for increased freedom in computing microdosimetric quantities and investigating energy deposition within $\mu \mathrm{m}$-sized targets. The MC method is a numerical technique that uses random sampling to simulate interactions of radiation with matter. 
The EGSnrc MC software is used to carry out all MC simulations in this thesis. $^{28}$ EGSnrc is capable of simulating photons and electrons down to $1 \mathrm{keV}$. EGSnrc simulates photon transport explicitly: photons interact via photoelectric interactions (dominant at low energies), Compton scattering (dominant at intermediate energies), electron/positron pair production (dominant at high energies) and Rayleigh scattering (does not result in energy transfer to electrons, unlike the previous three interaction types). Electrons and positrons lose energy as a result of inelastic collisions with atomic electrons (dominant at low energies; setting delta particles into motion) and radiative energy loss (i.e., bremsstrahlung and positron annihilation; dominant at high energies). Electrons also undergo elastic collisions with nuclei, resulting in a change in direction without any loss of energy.

As an electron slows down, it undergoes a large number of interactions that result in relatively small changes to the particle's energy and trajectory. Since it is not computationally feasible to simulate each of these interactions individually, the EGSnrc MC software uses the condensed history approach to simulate electron transport, which involves grouping together many interactions into a single step. ${ }^{29}$ At the end of each of these artificial steps, the particle's energy, direction of flight and position are updated to represent the sum of many small interactions using multiple scattering theory. However, EGSnrc switches to single scattering mode (the condensed history approach is not used) near boundaries between different regions of the simulation geometry. Because EGSnrc uses a class II condensed history MC approach, electron interactions resulting in delta particles or bremsstrahlung photons with energies greater than some user-defined energy threshold are simulated explicitly and the secondary electrons/photons are transported. In contrast, energy loss and secondary particle production are uncorrelated in a class I condensed history approach, and particles lose energy according to a predetermined 'grid' (there is no user-defined energy threshold). ${ }^{29,30}$

In addition to photon and electron transport, EGSnrc models atomic relaxations 
(that occur as a result of photon or electron interactions with atomic electrons), through Auger electron (dominant at low energies) and fluorescent photon (i.e., characteristic x-ray; dominant at high energies) emission. The "shower" of particles (e.g., photons and electrons) that are produced as a result of radiation interacting with matter is too complex to analyze analytically; the $\mathrm{MC}$ method is the only technique for obtaining a solution in most practical, non-simplified situations. ${ }^{28,30}$

\subsection{Radiation dosimetry on the cellular level}

Computational radiation dosimetry is used to calculate energy deposition resulting from radiotherapy and diagnostic radiology procedures. Traditionally, the human body is assumed to be water-equivalent for the purpose of simplifying these calculations. More recently, computational radiation dosimetry has become more sophisticated with departures from traditional water-based approaches towards advanced MC or other algorithms involving detailed computational models for radiotherapy and diagnostic radiology contexts. ${ }^{31-33}$ These virtual patient models typically involve mm-sized voxels or larger geometrical shapes containing macroscopic bulk tissue representing the volumeaverage of the underlying microscopic structure. However, understanding and predicting the biological effects of radiation requires knowledge of not only macroscopic dosimetry but also energy deposition on much shorter length scales. Cells and their constituents are generally considered relevant radiobiological targets. ${ }^{10,34}$ Cell death and genetic mutations can lead to tumour shrinkage, normal tissue damage and cancer induction. Therefore, cellular dosimetry is an important consideration for advancing our understanding of radiotherapy treatment outcomes and radiation protection studies. Recent research has demonstrated that cell doses can differ considerably from dose to water

and/or dose to bulk tissue, especially for lower incident photon energies, ${ }^{35}$ suggesting that underlying cellular structure and composition are important considerations for accurately determining biologically-relevant dose descriptors. 
The nucleus, containing DNA, is often considered the primary target for radiationinduced cell death. ${ }^{36,37}$ The most prevalent form of radiation-induced cell death is mitotic death, where chromosomal aberrations resulting from DNA strand breaks prevent cell division. ${ }^{10}$ Goodhead $^{37}$ suggests that DNA plus associated water molecules, which can undergo radiolysis resulting in free radical production and DNA damage, are the relevant targets. Recent work supports the importance of such $\sim$ nm-sized volumes within the nucleus. ${ }^{38}$ Cell death can also be triggered by damage to other targets such as the cell membrane and cytoplasm (which contains various organelles), resulting in apoptosis, or programmed cell death, regardless of whether or not the DNA has been damaged. ${ }^{34,39-41}$ Mitochondria have been shown to play an important role in radiation-induced cell death. ${ }^{42-44}$ Cell death can also be triggered by the bystander effect, whereby irradiated cells lead to manifestations of damage in unirradiated cells. ${ }^{22}$ However, radiation does not always result in cell death or senescence, although this is the desirable outcome in terms of avoiding cancer induction; genetic mutations can lead to malignancies. ${ }^{10}$

\subsection{Linking macro- and microscopic dose descriptors}

Due to limits on computational resources, simulation of radiation transport and energy deposition within detailed, multicellular models or on the molecular level is not always feasible, especially in the clinic. Macroscopic dose descriptors (based on computational models involving $\sim$ mm-sized voxels) such as dose-to-medium-in-medium $\left(D_{m, m}\right)$, doseto-water-in-medium $\left(D_{w, m}\right)$ or dose-to-water-in-water $\left(D_{w, w}\right)$ are commonly calculated

in clinical treatment planning. ${ }^{32,45} \mathrm{MC}$ and other model-based dose calculation algorithms (MBDCAs) generally compute $D_{m, m}$, which represents dose to a region or voxel in which tissue composition is assumed to be locally uniform (homogeneous bulk tissue) within a voxelized patient model. Given the radiobiological importance of the nucleus, and given that dose-to-nucleus $\left(D_{n u c}\right)$ can differ considerably from $D_{m, m},{ }^{35}$ conversion 
factors relating macro- $\left(e . g ., D_{m, m}\right)$ and microscopic $\left(e . g\right.$. , nuclear dose $\left.D_{n u c}\right)$ dose descriptors are potentially useful, and may be estimated using cavity theory (see e.g., Nahum ${ }^{46}$ ). Furthermore, cavity theory may provide a theoretical perspective helpful in understanding, predicting and verifying computational and experimental results. Small, intermediate and large cavity theories exist, with domains of applicability that depend on the size of the cavity (i.e., the nucleus) relative to the ranges of electrons set into motion by the incident photons.

Cavity theory conversion factors relating macroscopic dose descriptors to biologicallyrelevant, microscopic dose descriptors such as $D_{n u c}$ have been discussed in the context of the debate over the medium for dose specification for $\mathrm{MC}$ treatment planning (i.e., dose-to-water $D_{w, m}$ versus dose-to-medium $\left.D_{m, m}\right) .{ }^{32}$ Historically, radiotherapy treatment planning and treatment machine calibration has been based on dose-to-water. ${ }^{47}$ Therefore, $D_{w, m}$ or $D_{w, w}$ has traditionally been used in the clinic. Dosimetry protocols such as AAPM task groups 51 and 43 involve calculations carried out in water phantoms. ${ }^{45,48}$ In reality, the exact composition of the patient is unknown; given a CT number, the density and composition are not uniquely defined. ${ }^{49}$ Therefore, the exact nature of the medium $m$ is uncertain. Beyond dose to a water voxel (or voxel composed of water-like tissue) in medium, $D_{w, m}$ may represent dose to water molecules bound to DNA. ${ }^{37}$ Biologically relevant targets for cancer induction and tumour control are sometimes more radiologically similar to water than to the corresponding bulk tissue; ${ }^{31}$ this is true for skeletal dosimetry. ${ }^{50}$

On the other hand, given that $D_{m, m}$ is the quantity computed by MC treatment planning systems, converting to $D_{w, m}$ adds uncertainty: this extra step involves multiplication by the ratio of collisional stopping powers for water $w$ and the medium $m$; stopping powers are not known with absolute certainty. ${ }^{51}$ Furthermore, clinical uncertainties are often large compared to the difference between $D_{m, m}$ and $D_{w, m}$, at least for soft tissues. In the context of cellular dosimetry, organelles residing in the cytosol are 
the relevant biological targets, as discussed above. Conversion factors relating macroand microscopic dose descriptors should be based on a cavity theory approach that is appropriate for the target of interest's size relative to electron ranges, which depend on its density and elemental composition. Small cavity theory conversion factors (collisional stopping power ratios) are commonly used $;{ }^{47}$ however, they may not be appropriate in some low energy situations, where large or intermediate cavity theories are needed. ${ }^{52}$

\subsection{Current status of cell dosimetry research}

The importance of microscopic cell constituents such as the cytoplasm and nucleus as targets for radiation-induced cell damage has motivated recent studies using MC techniques to calculate doses to cellular targets, moving away from traditional waterbased calculations to MC models with varying levels of detail. ${ }^{35,53-56}$ Enger et al. ${ }^{55}$ modelled a single 7 m-radius spherical 'nucleus' cavity in a homogeneous tissue cube. Byrne et $a l .{ }^{56}$ modelled the nucleus and cytoplasm as two concentric spheres (radii 2 and $5 \mu \mathrm{m}$ ) suspended in water. Thomson et al. ${ }^{35}$ developed multicellular, microscopic tissue structure models using spherical cells (nucleus and cell radii 5 and $7.35 \mu \mathrm{m}$, respectively), a range of elemental compositions, and a cell number density typical of cancerous tissues, demonstrating the sensitivity of cell doses to surrounding cells and extracellular matrix (ECM). Incerti et $a l .{ }^{53}$ and Barberet et $a l .{ }^{54}$ developed voxelized models of human keratinocyte cells based on confocal microscopy and ion beam analysis, for single cells and 76-cell monolayers, respectively. Douglass et al. ${ }^{57}$ developed a multicellular model involving ellipsoidal cells with randomly selected positions, sizes and rotations.

While the research described above has demonstrated the effects of non-water media with different radiation sources from keV photons and electrons to $\mathrm{MeV}$ alpha particles, studies have typically focused on one cell size. Differing elemental compositions for cell constituents and surroundings (as well as use of some elemental compositions outside 
typical ranges identified by Thomson et al. ${ }^{35}$ ) limit direct comparisons between different publications. Thus, questions regarding the sensitivity of cytoplasm and nuclear doses to cellular compartment sizes, elemental compositions and level of detail for the surrounding geometry remain. Further, differences between cancerous and healthy cells affect cell-level energy deposition. ${ }^{35}$

Research toward connecting energy deposition within $\mu \mathrm{m}$ - or nm-sized targets with macroscopic dose descriptors (on which radiotherapy treatment plans and radiation protection studies are based) is ongoing. El Naqa et al. ${ }^{58}$ presented a general vision for a multi-scale modelling framework to take into account a range of temporal and spatial scales, involving physical, chemical, and biological effects. Focusing on physical effects, Thomson et al. ${ }^{35}$ investigated relations between macroscopic and microscopic (cell-level) dose descriptors, reporting that doses to subcellular targets and the corresponding bulk medium differ considerably for low energy photons $(<50 \mathrm{keV})$, citing differences of up to $23 \%$. These relations were investigated for one cell size, however, further research is needed to understand their dependance on cell size and morphology. Furthermore, these considerations are relevant for the ongoing debate (described above) of whether dose-to-water or dose-to-medium is more appropriate for model-based dose calculations, ${ }^{31}$ which has been reframed into a question (still unanswered) of which macroscopic dose descriptor best correlates with absorbed dose to biologically relevant targets. ${ }^{32}$ Beyond the goal of advancing radiation dosimetry and understanding radiobiological effects, research connecting macroscopic and microscopic views of energy deposition is relevant for prospective treatment modalities such as gold nanoparticle (GNP) radiosensitization. ${ }^{59-62}$

Regarding cavity theory studies, Carlsson Tedgren and Alm Carlsson ${ }^{63}$ classified water cavities (sizes from $1 \mathrm{~nm}$ to $1 \mathrm{~mm}$ ) in tissue as small, intermediate or large based on an ICT approach, considering source energies relevant for brachytherapy. Motivated by the potential radiobiological importance of small volumes of DNA-bound water lo- 
cated within the nucleus and the fact that a small water target is independent of cell type, Carlsson Tedgren and Alm Carlsson ${ }^{63}$ suggested that conversion of MBDCAcomputed dose-to-medium, $D_{m, m}$, to dose-to-water, $D_{w, m}$, via SCT may be "preferred" in the context of brachytherapy. For kilovoltage photon sources, Enger et al. ${ }^{55}$ compared SCT and LCT predictions of $D_{w, m}$ to MC-computed doses to nuclear cavities (7 $\mu \mathrm{m}$ radius) in otherwise homogeneous $(1 \mathrm{~cm})^{3}$ soft-tissue phantoms.

Villegas et al. ${ }^{64}$ (see also Villegas and Ahnesjö ${ }^{65}$ ) used MC techniques to investigate the microdosimetric spread within nucleus-sized cubic voxels in an all-water model, demonstrating that the dose threshold beyond which the specific energy distribution passes the Shapiro-Wilk test for normality depends on the target size and source energy. Cunha et $a .^{66}$ also investigated microdosimetric trends using an all-water model with cylindrical targets ranging in size from 0.1 to $10 \mu \mathrm{m}$. However, recent work has demonstrated the sensitivity of nuclear and cytoplasm doses to cellular and bulk tissue (non-water) elemental compositions, and microscopic details in the surrounding environment for kilovoltage photon energies. ${ }^{35}$ Thus, microdosimetric studies involving more detailed multicellular models are needed. Despite recent work in developing multicellular models, ${ }^{35,54,57,67}$ none of these studies involving multicellular models consider potential variation in energy deposition across a population of cells (i.e., the microdosimetric spread).

Mammography and Raman spectroscopy are two example scenarios where the stochastic nature of radiation is a potentially important consideration. On the microscopic level, the human breast contains epithelial cells that line mammary glands, adipocytes (i.e., lipid storage cells) and fibroblasts. Regarding radiation-induced cancers relevant for mammography risk assessment, epithelial cell malignancies are most common, ${ }^{68}$ with cell nuclei (which contain DNA) being considered important targets for cancer induction. ${ }^{10}$ Given that recent research has demonstrated considerable differences between macro- and microscopic dose descriptors for kilovoltage photon energies, ${ }^{35}$ 
and given that mammography uses kilovoltage photon sources, there may be considerable differences between macroscopic dose descriptors relevant for mammography (such as the mean glandular dose or dose to a glandular tissue voxel), and energy deposition in subcellular targets within the breast. A typical mammography exam delivers radiation dose to breast tissue on the order of several mGy. Therefore, mammography is a situation where microdosimetric considerations are potentially important for radiation risk assessment; the stochastic nature of radiation may result in considerable variation in energy deposition across a population of mammary epithelial cell nuclei. Furthermore, recent studies have used Raman spectroscopy to probe cell response to radiation using $\mu \mathrm{m}$-sized sampling volumes, and considering radiation doses $<1 \mathrm{~Gy}$. ${ }^{69,70}$ Raman spectroscopy can be used to assess radiosensitivity, as well as investigate radiation-induced damage to particular subcellular components based on knowledge of molecular vibration frequencies.

\subsection{Thesis purpose}

The purpose of this thesis is to use MC simulations to develop a better understanding of radiation transport and energy deposition on the cellular level. MC simulations are used to investigate the relationships between dose specified on a macroscopic scale $(\sim 1 \mathrm{~mm})$, and corresponding energy deposition in microscopic (cellular) targets such as the nucleus and cytoplasm. Cavity theory conversion factors are explored as a potential method for linking macro- and microscopic dose descriptors. The sensitivity of cellular energy deposition with respect to incident photon energy, dose level, and details of the microscopic tissue structure model (e.g., cell morphology, cell arrangement method, choice of media) will be explored. Models of various normal and cancerous human soft tissues are developed; these are referred to as 'multiscale' models because they combine varying levels of detail on different length scales, from the cellular level (microscopic modelling) to macroscopic tissue models. The importance of the microdosimetric spread 
in various scenarios is explored, including Raman spectroscopy and mammography.

The work presented in this thesis considers energy deposition in subcellular targets for monoenergetic incident photon energies from 20 to $370 \mathrm{keV}$ relevant for diagnostic radiology, brachytherapy, and out-of-field radiation in higher energy external beam radiotherapy treatments. A $120 \mathrm{kVp}$ x-ray photon spectrum, a ${ }^{60} \mathrm{Co}$ photon spectrum, and a $6 \mathrm{MV}$ medical linac photon spectrum are also considered, which are relevant for radiotherapy. Additionally, a $30 \mathrm{kVp}$ mammography x-ray photon spectrum is considered.

\subsection{Thesis outline}

A general description of MC simulation methods is given in chapter 2. In chapter 3, multicellular models of human soft tissues are developed, and are used to investigate the relationships between macroscopic and microscopic (i.e., cellular) dose descriptors for various kilovoltage incident photon energies; sensitivity to model parameters is explored. Chapter 4 features a survey of cavity theory approaches from the literature, which are applied in the context of cellular dosimetry; cavity theory is used to generate conversion factors relating $D_{n u c}$ and $D_{m, m}$. Multiscale models of human soft tissues are developed in chapter 5 , and are used to investigate energy deposition within populations of cells; in particular, the dependence of the microdosimetric spread on incident photon energy, dose level, and various model parameters is explored. Chapter 6 considers the stochastic nature of radiation transport and energy deposition in the context of Raman spectroscopy radiation response studies. In chapter 7, multiscale models of compressed breasts are used to investigate energy deposition in glandular tissues for mammography; both macro- and microscopic length scales are considered. A summary of the thesis, along with a description of future research possibilities is presented in chapter 8 . 


\section{Chapter 2}

\section{Methods}

This chapter outlines aspects that are common to all Monte Carlo simulations carried out in this thesis. A description of the Monte Carlo user-codes and associated transport parameters used herein are presented. Media definitions and corresponding radiological parameters, and a literature survey of human cell and nucleus sizes are also included. 


\subsection{Monte Carlo simulations using the EGSnrc software}

The EGSnrc user-codes egs_chamber, FLURZnrc, and egs_brachy are used to simulate radiation transport and energy deposition in a variety of scenarios. Statistical uncertainties are calculated according to the history by history approach. ${ }^{71}$

Most MC simulations are carried out using the EGSnrc user-code egs_chamber, which calculates energy deposition within small targets using variance reduction techniques to enhance calculation efficiency. ${ }^{72}$ The egs_chamber code makes use of the EGSnrc $\mathrm{C}++$ class library to define the simulation geometry and radiation source. ${ }^{73}$ In chapters 5, 6, and 7, a version of egs_chamber that has been modified to allow for scoring energy deposition in multiple regions is used. The following variance reduction techniques are used in chapters 3 and 4: cross section enhancement, range-based Russian roulette, and intermediate phase space scoring. cross section enhancement is not used in chapters 5, 6, or 7 since it was found to perturb resulting specific energy distributions.

The FLURZnrc user-code ${ }^{74}$ is used in chapter 4 to calculate electron and photon fluence spectra. These spectra are used to calculate spectrum-average mass energy absorption coefficient and stopping power ratios, for large and small cavity theory, respectively.

For egs_chamber and FLURZnrc simulations, the transport cutoff and production threshold for the kinetic energy of electrons and photons is $1 \mathrm{keV}$. The Continuous Slowing Down Approximation (CSDA) range of a $1 \mathrm{keV}$ electron in water is $R_{C S D A} \simeq 0.04 \mathrm{\mu m}^{1}$, which is small compared to the smallest dimension encountered in the simulation geometries considered herein. The XCOM photon cross section

\footnotetext{
${ }^{1} \mathrm{CSDA}$ ranges are calculated according to the methods outlined in ICRU report $37,{ }^{75}$ using stopping powers from the NIST ESTAR database ${ }^{76}$
} 
database $^{77}$ and the NRC bremsstrahlung cross section database ${ }^{28}$ are used. Regarding uncertainties on the photon cross sections, Hubbell ${ }^{78}$ points out that a 'rough guide' to photon cross section uncertainties is given by Cullen et al. ${ }^{79}$ who give maximum photoionization (e.g., photoelectric interaction) cross section uncertainties of $5 \%$ for photon energies between 1 and $10 \mathrm{MeV}$, for the EPDL97 library (equivalent to the NIST XCOM database used herein). Seeking to understand the influence of photon cross section uncertainties on dose-rate constants, Rivard et al. ${ }^{45}$ state that photoelectric cross sections of the DLC-146 library (also equivalent to the NIST XCOM database) and the DLC-99 library (an older version) differ by $\sim 2 \%$; this difference is used as a photon cross section uncertainty estimate. Furthermore, Seltzer and Berger ${ }^{80}$ state that NIST bremsstrahlung cross sections have uncertainties $\sim 10 \%$ or less for electron energies $\leq 50 \mathrm{MeV}$; the NRC bremsstrahlung cross section database used herein is based on the NIST database, but includes electron-electron bremsstrahlung contributions to the cross section. ${ }^{28}$ Additionally, Rayleigh scattering and electron impact ionization are turned on. Given the microscopic features included in the simulation geometries considered herein, the high resolution random number generator option is used. The other transport parameters are EGSnrc defaults. Robustness of simulation results to changes in transport parameters are discussed in appendix A.

The egs_brachy user-code ${ }^{81,82}$ is used in chapter 7 to score dose in segmented breast tissue phantoms consisting of a three-dimensional array of $(0.5 \mathrm{~mm})^{3}$ voxels. This usercode also uses the EGSnrc C++ class library. Transport parameters for the egs_brachy simulations are mostly the same as those described above for the egs_chamber and FLURZnrc simulations. However, for egs_brachy, the electron transport threshold is set to $1.5 \mathrm{MeV}$ so that no electron transport is simulated. The mammography spectrum considered in chapter 7 includes photon energies up to $30 \mathrm{keV}$; resulting photoelectrons would have $R_{C S D A} \simeq 18 \mu \mathrm{m}$ in water. This range is small compared to the sizes of the voxels in the segmented phantom, which suggests that simulating electron transport is 
unnecessary. Furthermore, in egs_brachy, a tracklength estimator is used to score dose. The tracklength estimator assumes that photons deposit energy continuously as they traverse voxels, while in reality they interact at discrete locations. The tracklength estimator makes use of mass energy absorption coefficients to score collision kerma, which is equivalent to the dose, assuming that charged particle equilibrium (CPE) exists. CPE describes the situation where the number of electrons coming to rest in a given volume is the same as the number set in motion by the incident photon beam. ${ }^{83}$ Comparison tests showed that the tracklength estimator gave the same results as interaction scoring, within statistical uncertainty for the simulation geometry and source spectrum considered in chapter 7 (results not shown).

All MC simulations require a user-defined simulation geometry. Particles generated by a user-defined radiation source are transported throughout this geometry, which can be constructed using a grid of voxels, or by combining various objects (e.g., spheres, cubes, planes, etc.) to define boundaries of regions where energy deposition can be scored. The user must also specify the elemental compositions and densities of the media that fill the various regions. The media used throughout this work are described in the following section. A literature survey of human cell and nucleus sizes is also presented, which is used to motivate choices of subcellular compartment sizes used in the MC simulations presented in this thesis.

\subsection{Media definitions}

Two normal and three cancerous human, soft, bulk tissues are considered in chapters 3, 4, and 5. The elemental compositions and mass densities $\rho$ of mammary gland $\left(\rho=1.02 \mathrm{~g} / \mathrm{cm}^{3}\right)$ and muscle $\left(\rho=1.05 \mathrm{~g} / \mathrm{cm}^{3}\right)$ are taken from Woodard and White ${ }^{84}$ and ICRU Report 44, ${ }^{85}$ respectively, while the compositions of the three cancerous tissues (melanoma, adenoidcystic carcinoma and squamous cell lung carcinoma) are obtained from Maughan et al., ${ }^{86}$ assuming a mass density of $1.04 \mathrm{~g} / \mathrm{cm}^{3} .{ }^{35}$ In chapter 
5 , three of the five bulk tissues are modified slightly as described in the text of that chapter. In chapters 3,4 , and 5 , water is assumed to exist at $22^{\circ} \mathrm{C}$ so that its density is taken to be $0.998 \mathrm{~g} / \mathrm{cm}^{3}{ }^{45}$ In chapter 6 , unit density water is used throughout the simulation geometry, for simplicity. In chapter 7, breast dosimetry for mammography is considered, and media given in Sechopoulos et al. ${ }^{33}$ are used; therefore, unit density water is used in this chapter as well.

The nucleus and cytoplasm elemental compositions are based on published data on intracellular water content, ${ }^{87-91}$ inorganic content, ${ }^{91,92}$ and organic content ${ }^{93}$ of animal cells. Nucleus and cytoplasm models 'a', 'b', 'c' and 'd' have different water content, but the same dry inorganic elemental composition, given by mouse tumour mammary adenocarcinoma as measured by Cameron et al. ${ }^{92}$ Models 'b', 'e', 'f' and 'g' have the same water content (the nucleus and cytoplasm are $75 \%$ and $70 \%$ water by mass, respectively) but different inorganic content. The inorganic content of composition 'e' is obtained from normal epithelial cells of a leech; 91 for models ' $\mathrm{f}$ ' and ' $\mathrm{g}$ ', it is obtained from normal hepatocyte cells of a rat, and normal lactating mammary epithelium of a mouse, respectively. ${ }^{92}$ Nucleus and cytoplasm mass densities are taken as $1.06 \mathrm{~g} / \mathrm{cm}^{3} .{ }^{35}$ The methods used to develop these cellular elemental compositions are described by Thomson et al. ${ }^{35}$ In chapter 7 , lipid storage cells (adipocytes) having an additional subcellular compartment containing lipid, are considered; lipid elemental composition and density is taken from ICRP publication $89 .{ }^{94}$

Mass energy absorption coefficients and unrestricted mass collisional stopping powers (these radiological parameters are obtained from the EGSnrc user-codes g and examin, respectively ${ }^{28}$ ) for the seven cellular elemental compositions are presented in figure 2.1. A comparison of radiological parameters of the original and modified bulk tissues is presented in figure 2.2. Elemental compositions, water content, and mass densities of the bulk tissues, cellular media and lipid are shown in table 2.1. 

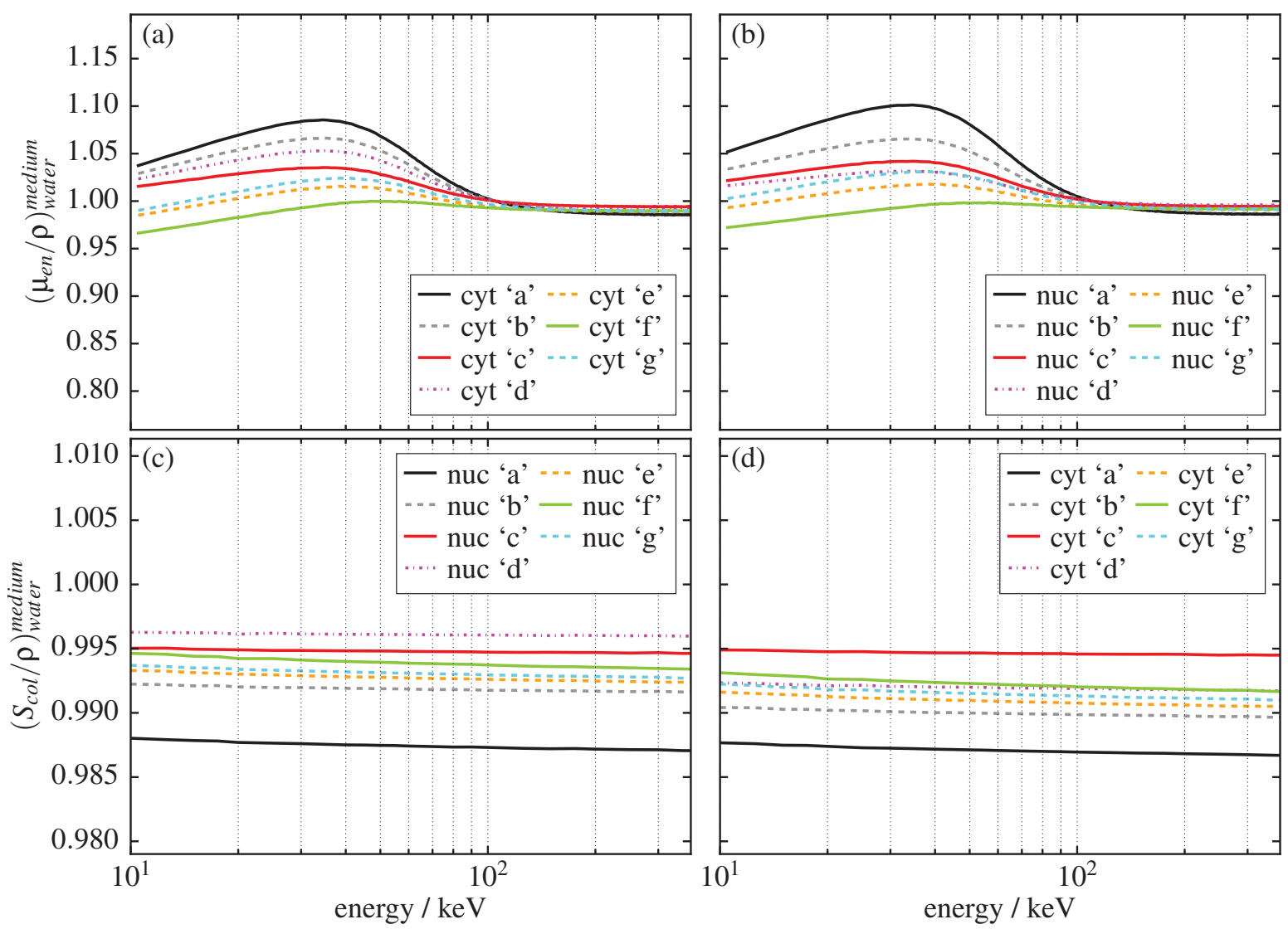

Figure 2.1: (a,b) Mass energy absorption coefficient $\mu_{e n} / \rho$ and $(\mathrm{c}, \mathrm{d})$ unrestricted mass collisional stopping power $S_{\text {col }} / \rho$ ratios in cellular media with respect to water $(\rho=$ $0.998 \mathrm{~g} / \mathrm{cm}^{3}$ ) are plotted as a function of energy. 'nuc' and 'cyt' refer to the nucleus and cytoplasm media, respectively. 

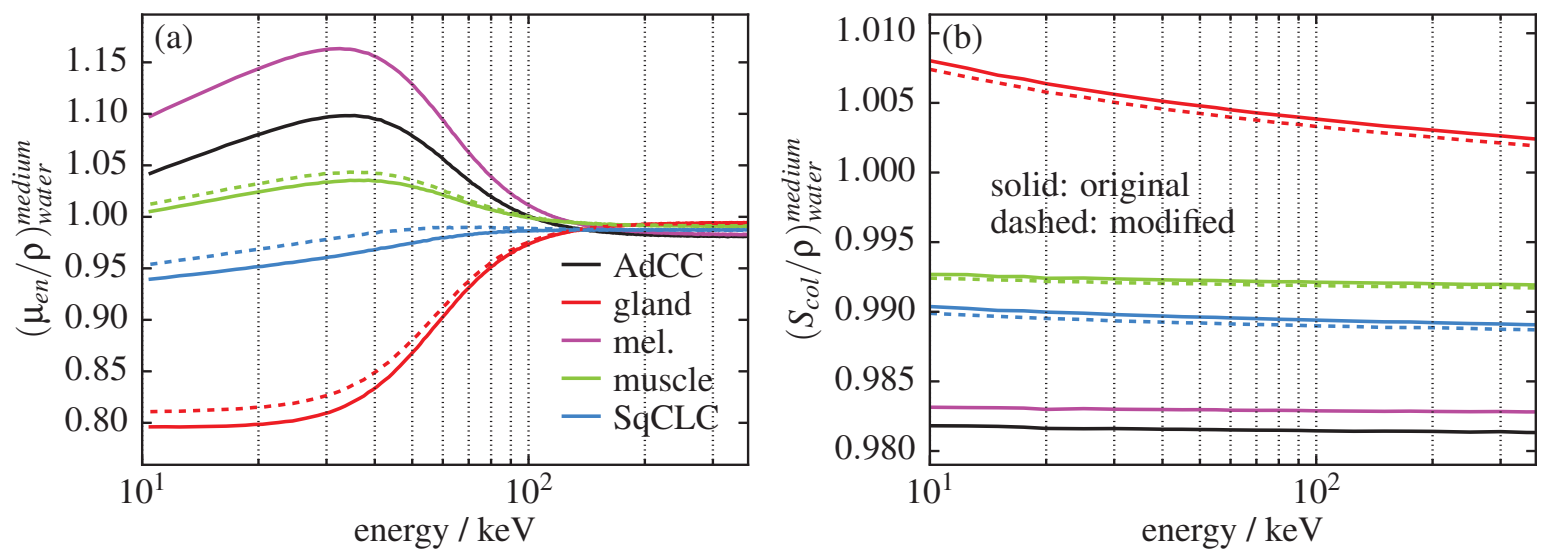

Figure 2.2: (a) Mass energy absorption coefficient $\mu_{e n} / \rho$ and (b) unrestricted mass collisional stopping power $S_{\text {col }} / \rho$ ratios for bulk tissues with respect to water $\left(\rho=0.998 \mathrm{~g} / \mathrm{cm}^{3}\right)$ are plotted as a function of energy. Five bulk tissues are considered: adenoidcystic carcinoma ('AdCC'), mammary gland ('gland'), melanoma ('mel'), muscle and squamous cell lung carcinoma ('SqCLC'). The original (modified) bulk tissues are indicated by the solid (dashed) lines. 


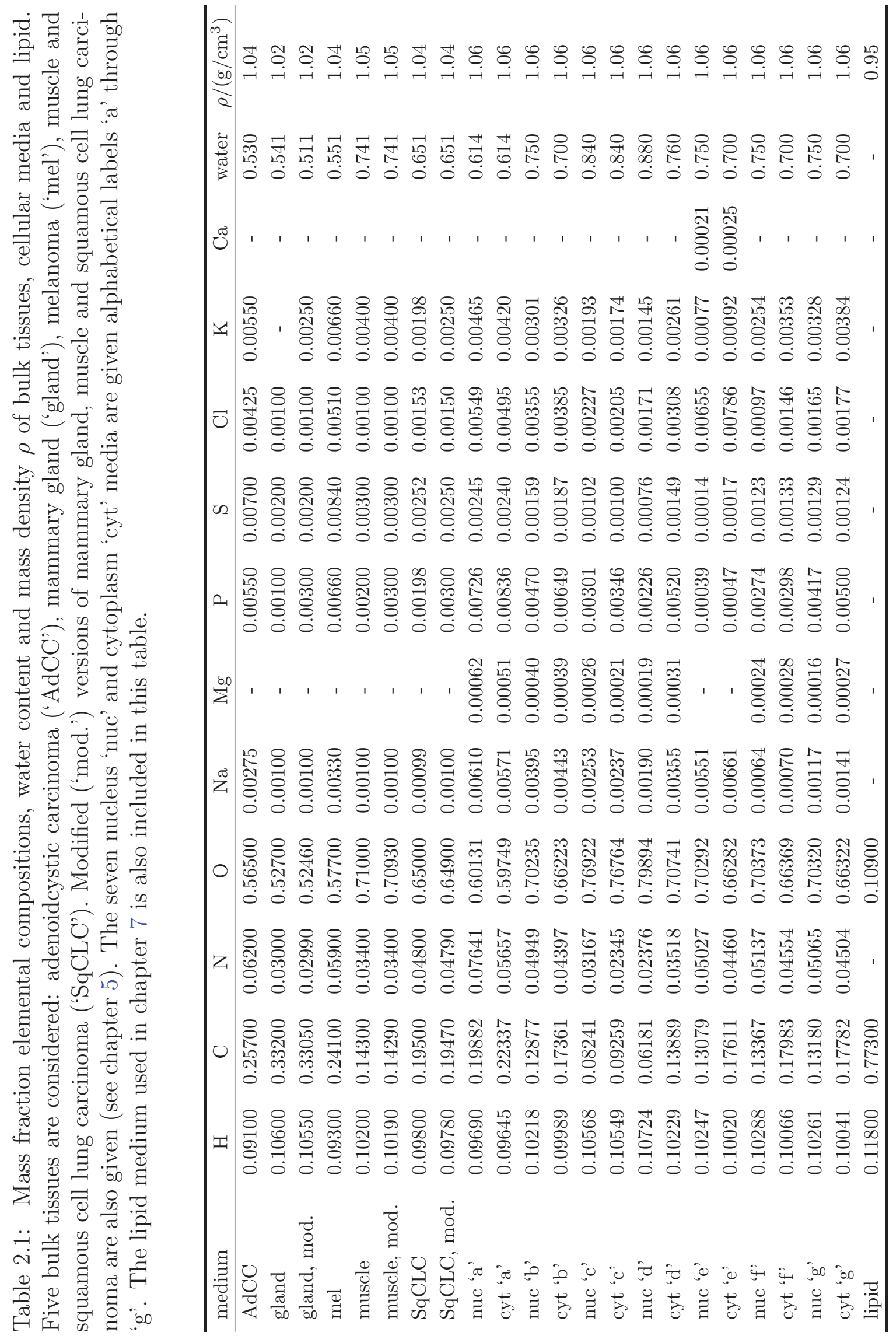




\subsection{Literature survey of cell and nucleus sizes}

A survey of the scientific literature was carried out to determine typical sizes of human cells and nuclei - summarized in table 2.2. While cells and nuclei are often approximated as spheres with sizes characterized by their radii $\left(r_{\text {cell }}\right.$ and $r_{n u c}$, respectively, presented in table 2.2), cells exist in a variety of shapes. For example, Tan et al. ${ }^{95}$ studied the cellular morphology of ductal carcinoma in situ (DCIS) of the breast using the ratio of minimum to maximum Feret diameters (which is the distance between parallel planes bounding the object), to quantify the ellipticity of the cell. For the 64 cases of DCIS investigated, the average Feret ratio was found to be 0.728 with a standard error on the mean of 0.005 , and was found to exhibit no significant variation with nuclear grade (an indicator of disease progression), unlike the nuclear radius, which increased with nuclear grade (see table 2.2). The data from Koss and Melamed ${ }^{96}$ demonstrate the general trend that the relative size of the nucleus compared to the size of the cell is larger for malignant cells: the nuclear radius for normal cells of the cervix $(3.38 \mu \mathrm{m})$ is small compared to that of dysplastic or malignant (in situ or invasive) cells of the cervix (4.89 to $7.26 \mu \mathrm{m})$. Out of these four categories of cervical cells, those from

a dysplastic (precancerous) growth in the cervix have the largest nuclei. Also, the cell radius becomes smaller as the cell progresses from normal to dysplastic to in situ malignant to invasive malignant. 
Table 2.2: Summary of human cell and nuclear radii $\left(r_{\text {cell }}\right.$ and $\left.r_{n u c}\right)$ from the literature; entries are listed from smallest to largest $r_{\text {cell }}$ ( or $r_{\text {nuc }}$ if $r_{\text {cell }}$ is not specified).

* Radius obtained assuming the cell volume is the sum of cytoplasm and nuclear volumes.

- Radius obtained from the volume or cross sectional area, assuming a sphere.

${ }^{\dagger}$ cross sectional areas are obtained from figures 5 and 6 in Watanabe et al. ${ }^{97}$

\begin{tabular}{|c|c|c|}
\hline$r_{\text {cell }} / \mu \mathrm{m}$ & $r_{n u c} / \mu \mathrm{m}$ & Type of cell (reference) \\
\hline 3.75 & - & Erythrocytes (Bain ${ }^{98}$ ) \\
\hline $3.988 \pm 0.905$ & - & Circulating prostate tumour cells (Park et al..$^{99}$ ) \\
\hline 5 to 8 & - & Lymphocytes (Bain ${ }^{98}$ ) \\
\hline 5 to 8.5 & - & Granulocytes (Bain ${ }^{98}$ ) \\
\hline 6 to 10 & - & Myeloblasts (Bain ${ }^{98}$ ) \\
\hline $6.6 \pm 0.9^{\star \Delta}$ & $5.4 \pm 0.5^{\star \Delta}$ & Average of four keratinocyte cells (Incerti et al..$^{53}$ ) \\
\hline $6.693 \pm 1.27$ & - & Cultured prostate cancer cells (Park et al. ${ }^{99}$ ) \\
\hline $7.174 \pm 0.001^{\star}$ & - & Glioblastoma (right temporal lobe) (Wéra et al. ${ }^{100}$ ) \\
\hline $7.3 \pm 1.0$ & - & Heart muscle cell, right ventricle (Adler et al. ${ }^{101}$ ) \\
\hline $8.0 \pm 1.1$ & - & Heart muscle cell, left ventricle (Adler et al. ${ }^{101}$ ) \\
\hline $8.1 \pm 1.2^{\dagger \triangleleft}$ & $3.51 \pm 0.27^{\dagger \mathbf{\Delta}}$ & Liver cancer cell (Watanabe et al. ${ }^{97}$ ) \\
\hline $8.4 \pm 1.5$ & $4.89 \pm 0.80$ & Invasive cancer of the cervix (Koss and Melamed ${ }^{96}$ ) \\
\hline $8.9 \pm 0.8$ & $5.8 \pm 0.5$ & Average of six breast cancer cell lines (Cai et al. ${ }^{102}$ ) \\
\hline 9.2 & 6.4 & Stage III non-small cell lung cancer (Lee et al. ${ }^{103}$ ) \\
\hline 10 to 15 & 5 to 7.5 & Endothelial cells $\left(\right.$ Bain $\left.^{98}\right)$ \\
\hline $10.4 \pm 1.6$ & $5.84 \pm 0.83$ & In situ cancer of the cervix (Koss and Melamed ${ }^{96}$ ) \\
\hline $10.9 \pm 0.6^{\dagger \mathbf{\Delta}}$ & $3.25 \pm 0.10^{\dagger \mathbf{\Delta}}$ & Normal liver cell (Watanabe et al. ${ }^{97}$ ) \\
\hline $18.4 \pm 2.8$ & $7.26 \pm 0.79$ & Dysplasia of the cervix (Koss and Melamed ${ }^{96}$ ) \\
\hline $22.5 \pm 2.1$ & $3.38 \pm 0.60$ & Normal cervix (Koss and Melamed ${ }^{96}$ ) \\
\hline- & 2.5 to 5.0 & Non-dysplastic epithelial cells (Backman et al. ${ }^{104}$ ) \\
\hline- & $2.82 \pm 0.06^{\mathbf{\Lambda}}$ & Normal breast cell (Pienta and Coffey ${ }^{105}$ ) \\
\hline- & $\begin{array}{l}3.2 \pm 0.1^{\mathbf{\Delta}} \\
3.61 \pm 0.09^{\mathbf{\Delta}} \\
4.2 \pm 0.1^{\mathbf{\Delta}}\end{array}$ & $\begin{array}{l}\text { Infiltrating ductal carcinoma of the breast: with recurrence, no } \\
\text { recurrence and metastatic lymph node (Pienta and Coffey }{ }^{105} \text { ) }\end{array}$ \\
\hline- & $3.57 \pm 0.09^{\mathbf{\Delta}}$ & Intraductal breast cancer (Pienta and Coffey ${ }^{105}$ ) \\
\hline- & $4.1 \pm 0.1$ & Small-cell lung carcinoma (Lee et al. ${ }^{106}$ ) \\
\hline- & $\begin{array}{ll}3.75, & 3.90 \\
4.79^{\mathbf{\Delta}} & \end{array}$ & $\begin{array}{l}\text { Ductal carcinoma in situ of the breast, nuclear grades } 1,2 \text { and } 3 \\
\text { (Tan et al. } .^{95} \text { ) }\end{array}$ \\
\hline- & $4.3 \pm 0.1^{\wedge}$ & $\begin{array}{l}\text { Malignant lymph node cells resulting from breast cancer metas- } \\
\text { tasis (Pienta and Coffey }{ }^{105} \text { ) }\end{array}$ \\
\hline- & $5.06 \pm 0.06$ & Glioblastoma cells (Nafe et al. ${ }^{107}$ ) \\
\hline- & $6.4 \pm 0.2$ & Lung carcinoma other than small-cell (Lee et al. ${ }^{106}$ ) \\
\hline- & $7.88^{\mathbf{4}}$ & Average of 76 keratinocyte cells (Barberet et al. ${ }^{54}$ ) \\
\hline- & 10.0 & Dysplastic epithelial cells (Backman et al. ${ }^{104}$ ) \\
\hline
\end{tabular}




\section{Chapter 3}

\section{Monte Carlo study of macro- and micro- scopic dose descriptors for kilovoltage cel- lular dosimetry}

Detailed, multicellular models of normal and malignant human soft tissues are developed. Monte Carlo simulations enable exploration of cellular dose variations with incident photon energy, cell/nucleus size, choice of media, and level of detail in the microscopic tissue structure. Relations between macroscopic and cellular doses are investigated, including discussion related to the dose-to-water versus dose-to-medium debate for Monte Carlo treatment planning. 


\subsection{Introduction}

In this chapter, we develop representative multicellular models of normal and cancerous human soft tissues and use these to explore the sensitivity of cell-level energy deposition to model details, as well as to investigate relationships between macroscopic and microscopic dose descriptors. Drawing on data from a scientific literature review, various elemental compositions representative of normal and cancerous animal tissues, and realistic subcellular compartment sizes are considered. Dose-to-water and dose-to-medium (macroscopic dose descriptors) are investigated as potential surrogates for nuclear and cytoplasm doses (microscopic dose descriptors). Incident photon energies between 20 and $370 \mathrm{keV}$ are considered. At these energies, electron ranges are comparable to cellular length scales, and there are pronounced differences between the radiological parameters of the soft tissue media considered herein, resulting in fluence and energy absorption characteristics that are strongly medium-dependent.

\subsection{Methods}

\subsubsection{Development of microscopic tissue structure models}

Each cell is modelled as a spherical nucleus surrounded by a concentric spherical cytoplasm. Based on the results of the literature survey (table 2.2), three cell radii and nine nuclear radii are investigated, as shown in table 3.1. For multicellular tissue models, cells arranged in a hexagonal lattice with a minimum cell separation of $2.06 \mu \mathrm{m}$ are embedded in an ECM. For this intercellular spacing and for the selected values of $r_{\text {cell }}$, the resulting cell number densities and ECM volume fractions (table 3.1) are consistent with values reported in the published literature, e.g., number densities of $2.63 \times 10^{8}$ cells per $\mathrm{cm}^{3}$ for human breast tumour tissue, ${ }^{108}$ and $5.13 \times 10^{8}$ cells per $\mathrm{cm}^{3}$ for human 
melanoma tissue; ${ }^{109}$ ECM volume fractions ranging from $30 \%$ to $60 \% .108,110,111$

Table 3.1: Parameters of the microscopic tissue structure model involving spherical cells (radius $\left.=r_{\text {cell }}\right)$ with concentric spherical nuclei $\left(\right.$ radius $\left.=r_{n u c}\right) . V_{\text {nuc }} / V_{\text {cell }}$ is the fraction of the cell volume occupied by the nucleus. A cluster of 13 cells is embedded in a sphere of ECM with radius $r_{E C M}$; see section 3.2.2 and figure 3.1. The fraction of the total volume occupied by ECM and the cell number density are indicated.

\begin{tabular}{llllll}
\hline$r_{\text {cell }} / \mu \mathrm{m}$ & $r_{\text {nuc }} / \mu \mathrm{m}$ & $V_{\text {nuc }} / V_{\text {cell }}$ & $r_{E C M} / \mu \mathrm{m}$ & $\begin{array}{l}\text { ECM volume } \\
\text { fraction }\end{array}$ & $\begin{array}{l}\text { cell number density/ } \\
\mathrm{cm}^{-3}\end{array}$ \\
\hline 5 & $2,3,4$ & $0.064,0.216,0.512$ & 18.09 & 0.578 & $8.06 \times 10^{8}$ \\
7.35 & $4,5,6$ & $0.161,0.315,0.544$ & 25.14 & 0.500 & $3.00 \times 10^{8}$ \\
10 & $7,8,9$ & $0.343,0.512,0.729$ & 33.09 & 0.448 & $1.32 \times 10^{8}$ \\
\hline
\end{tabular}

Five normal and cancerous bulk tissues, as well as seven nucleus and cytoplasm media (labelled 'a' through 'g') are considered. Elemental compositions, water content, and mass densities of these media, along with stopping powers and energy absorption coefficients are presented in table 2.1 and figure 2.1, respectively. The elemental composition and mass density of the ECM are determined by requiring that the average composition (weighted by mass) and mass density of the cells and surrounding ECM are approximately equal to that of the bulk tissue, resulting in a unique ECM definition for each bulk tissue, cell/nucleus size combination and cytoplasm/nucleus elemental composition.

\subsubsection{Monte Carlo simulations}

MC simulations are carried out using the EGSnrc user-code egs_chamber, using a variety of variance reduction techniques to enhance calculation efficiency. ${ }^{28,72,73}$ The radiation source is a parallel beam of monoenergetic photons; five energies are considered: 20, 30, 50, 90 and $370 \mathrm{keV}$. The beam has a circular cross section with a radius of $1 \mathrm{~cm}$.

Schematic simulation geometries are depicted in figure 3.1. The cell cluster model consists of 13 cells and dose is scored in the nucleus $\left(D_{\text {nuc }}\right)$ and cytoplasm $\left(D_{\text {cyt }}\right)$ of the central cell (figures 3.1(b) and 3.1(c)). The simpler geometries considered are also 
illustrated in figure 3.1: (d) $D_{n u c}^{1}\left(D_{c y t}^{1}\right)$ is the dose to the nucleus (cytoplasm) of a single cell; (e) $D_{n u c, m}$ is the dose to a single nucleus; (f) $D_{w, m}$ is the dose to a nucleus-sized water cavity; (g) $D_{m, m}\left(D_{m, m}^{c}\right)$ is the dose to the nucleus (cytoplasm) of a single cell

of bulk tissue, and (h) $D_{w, m}^{c}$ is the dose to the cytoplasm of a single cell composed of water. Each of these geometries is situated at the centre of an otherwise homogeneous $(2 \mathrm{~cm})^{3}$ bulk tissue phantom consisting of adenoidcystic carcinoma, mammary gland, melanoma, muscle or squamous cell lung carcinoma tissue. With 5 energies, 5 bulk tissues, 7 cytoplasm/nucleus elemental compositions (table 2.1), and 9 cell/nucleus size combinations (table 3.1), a total of up to 1575 simulations are carried out for each of the 6 simulation geometries (figure 3.1). Statistical uncertainties $(1 \sigma)$ on energy deposition are $<0.91 \%$, with an average of $0.16 \%$ over all results. Simulation results are analysed to investigate how cellular dose descriptors are influenced by the microscopic details in the surrounding environment (section 3.3.1), how cell morphology affects energy deposition (section 3.3.2), and how macroscopic dose descriptors (dose-to-medium and dose-to-water) compare to microscopic dose descriptors (section 3.3.3).

\subsection{Results}

\subsubsection{Surrounding geometry effects on nuclear and cytoplasm doses}

The microscopic details of the surrounding environment affect nuclear and cytoplasm doses, as demonstrated by the MC simulation results for 20, 30 and $50 \mathrm{keV}$ sources summarized in figure 3.2. Deviations of $D_{n u c, m} / D_{n u c}$ from unity (figure 3.2(a)) demonstrate the effect of the local cytoplasm, ECM and surrounding cells on nuclear dose. Variations in $D_{n u c, m} / D_{n u c}^{1}$ illustrate the effect of the local cytoplasm alone (figure $3.2(\mathrm{~b})$ ), while variations in $D_{n u c}^{1} / D_{n u c}$ and $D_{c y t}^{1} / D_{c y t}$ indicate the sensitivity of nuclear and cytoplasm doses to the presence of the ECM and surrounding cells (figures 3.2(c) and 3.2(d)). 

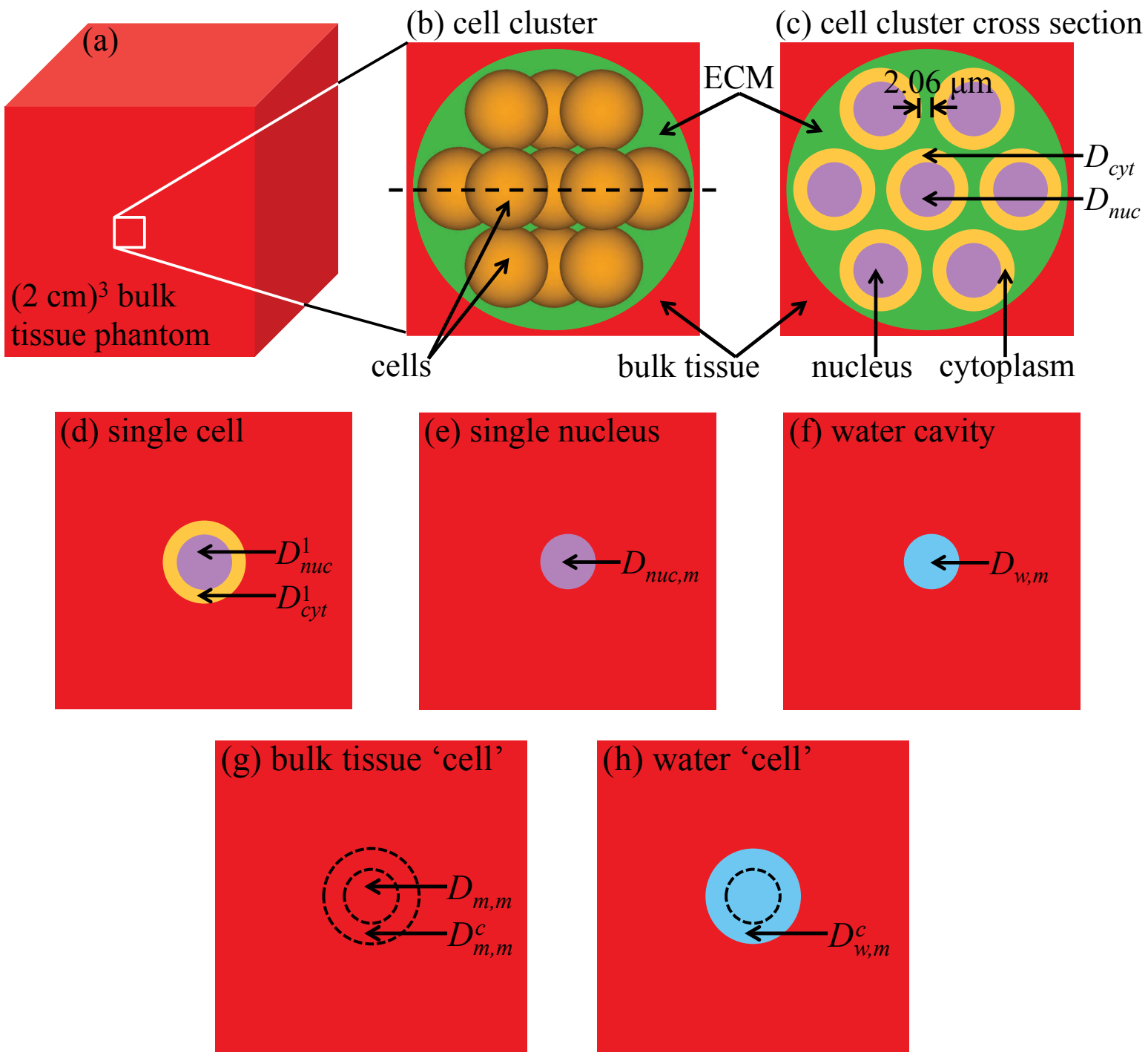

Figure 3.1: (a) Diagram of the $(2 \mathrm{~cm})^{3}$ cubic bulk tissue phantom. The multicellular model shown in (b) consists of 13 cells arranged in a hexagonal lattice. The dashed line indicates the location of the cross section through the centre of the cell cluster shown in (c): the cells are embedded in a sphere of extracellular matrix (ECM). Cross sections of other simulation geometries are also illustrated for (d) a single cell, (e) a single nucleus, (f) a single nucleus-sized water cavity, (g) a single cell of bulk tissue, and (h) a single cell composed of water; each of these is embedded in a bulk tissue phantom as shown in (a). Dose is scored in the nucleus or cytoplasm volume, as indicated. 

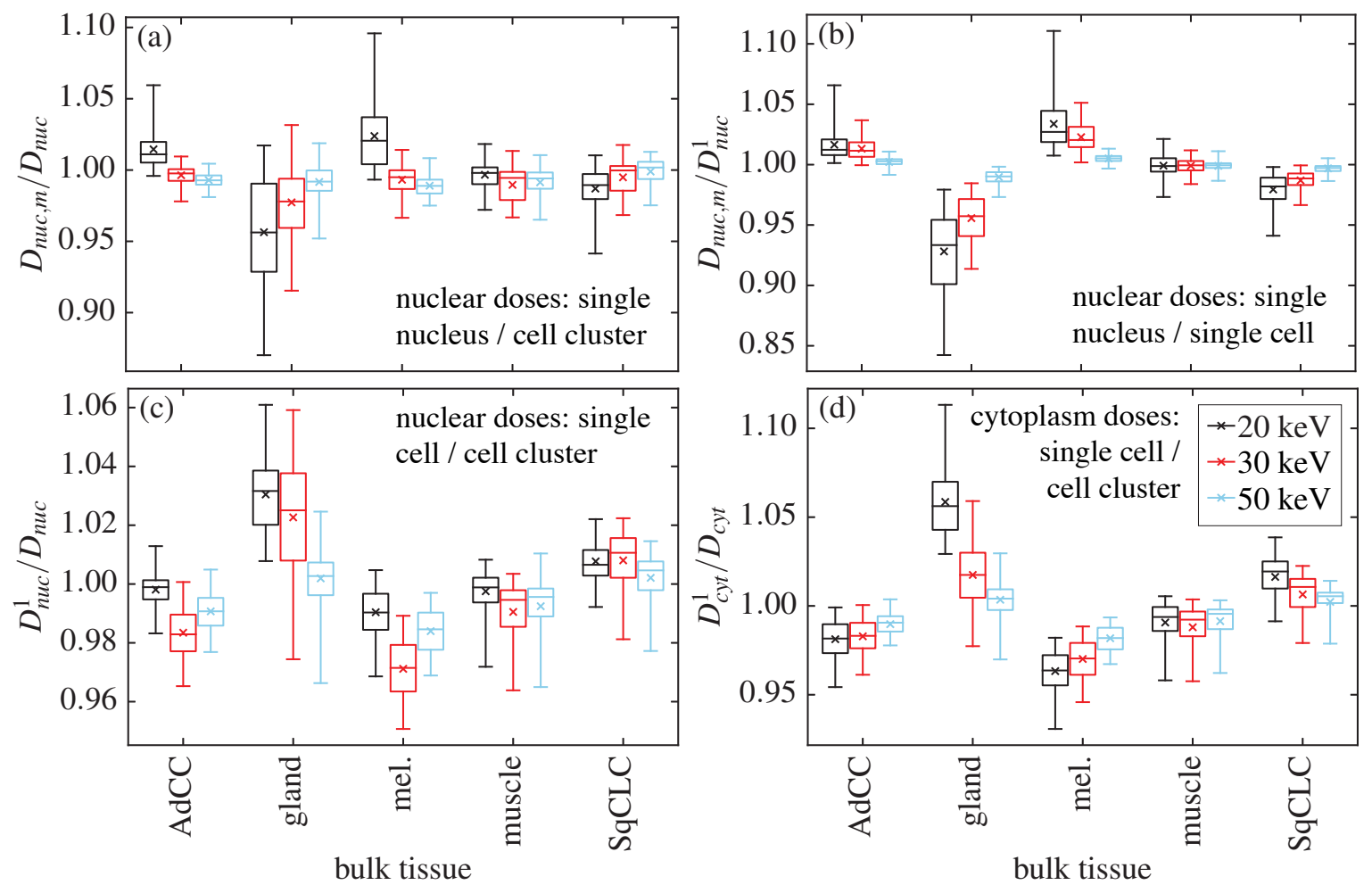

Figure 3.2: Summary of results for (a) $D_{n u c, m} / D_{n u c}$, (b) $D_{n u c, m} / D_{n u c}^{1}$, (c) $D_{n u c}^{1} / D_{n u c}$ and (d) $D_{c y t}^{1} / D_{c y t}$ for 20,30 and $50 \mathrm{keV}$ incident photons. Whiskers extend between the minimum and maximum values of the set of results for the nine cell/nucleus size combinations and seven cytoplasm/nucleus elemental compositions; box extends between the upper and lower quartiles; its horizontal line indicates the median, and the ' $x$ ' symbol indicates the mean value. Five bulk tissues are plotted on the horizontal axis: adenoidcystic carcinoma ('AdCC'), mammary gland ('gland'), melanoma ('mel'), muscle and squamous cell lung carcinoma ('SqCLC'). 
In general, the largest discrepancies between nuclear doses for a single nucleus $\left(D_{n u c, m}\right)$, single cell $\left(D_{n u c}^{1}\right)$, and central nucleus within a cell cluster $\left(D_{n u c}\right)$ are observed at the lower incident photon energies: the largest deviations from unity are $16 \%$ for $D_{n u c, m} / D_{n u c}^{1}$ when $\left(r_{\text {cell }}, r_{\text {nuc }}\right)=(5,2) \mu \mathrm{m}, 13 \%$ for $D_{n u c, m} / D_{\text {nuc }}$ when $\left(r_{\text {cell }}, r_{n u c}\right)=$ $(5,2) \mu \mathrm{m}$, and $6 \%$ for $D_{n u c}^{1} / D_{n u c}$ when $\left(r_{\text {cell }}, r_{n u c}\right)=(5,4) \mu \mathrm{m}$, all for mammary gland with $20 \mathrm{keV}$ incident photons. For some bulk tissues and cell models, deviations from unity for $D_{n u c}^{1} / D_{n u c}$ at $30 \mathrm{keV}$ are larger than those at $20 \mathrm{keV}$ (figure $3.2(\mathrm{c})$ ). These results stem from secondary electron ranges relative to the dimensions of the microscopic geometry, coupled with media cross sections. For the lower-energy sources, secondary electrons have CSDA ranges comparable to cell sizes, e.g., $R_{C S D A}$ is $8.6 \mu \mathrm{m}(20 \mathrm{keV})$ and $17.6 \mu \mathrm{m}(30 \mathrm{keV})$ for electrons in unit density water (CSDA ranges are comparable for water, soft tissues, and the cellular media considered herein). With the tortuous path of the slowing electron, a considerable fraction of the nuclear dose is due to electrons originating within the nucleus itself or its surroundings; dose descriptors then depend strongly on the microscopic details of the simulation geometry. At the lower energies, photoelectric interactions dominate over incoherent (Compton) scattering. Photoelectric cross sections are sensitive to atomic number and vary among the media considered herein (manifested as variations in mass-energy absorption coefficients $\mu_{e n} / \rho$ - see figure 2.1). Sensitivity to the surrounding geometry (i.e., deviations in $D_{n u c}^{1} / D_{\text {nuc }}$ from unity in figure $3.2(\mathrm{c}))$ persists up to the $50 \mathrm{keV}$ source energy $\left(R_{C S D A}=43 \mu \mathrm{m}\right.$ for $50 \mathrm{keV}$ electron in water).

Differences between the dose to the central nucleus in the cluster $\left(D_{\text {nuc }}\right)$ and nuclear doses for a single nucleus $\left(D_{n u c, m}\right)$ or single cell $\left(D_{n u c}^{1}\right)$ are at most $3 \%$ for 90 and $370 \mathrm{keV}$ sources (results not shown). For these higher-energy sources, electron ranges are large compared to the size of the cell cluster. Incoherent interactions dominate photoelectric events at these higher energies, and the cross sections of the former exhibit relatively little variation among the media investigated. Thus, the details of the surrounding 
geometry have a relatively small effect on nuclear doses (compared with the lowerenergy sources).

At 20 and $30 \mathrm{keV}$, for the same cell radius $\left(r_{\text {cell }}\right)$, differences between $D_{n u c, m}$ (dose to a single nucleus) and $D_{n u c}^{1}$ (nuclear dose for a single cell) increase with decreasing nuclear radius $\left(r_{n u c}\right)$, due to the increased 'thickness' of cytoplasm (results not shown). The trends observed for $D_{n u c, m} / D_{n u c}^{1}$ are similar to those exhibited by $D_{n u c, m} / D_{n u c}$ (where $D_{n u c}$ is the dose to the central nucleus of the cell cluster) as a function of cytoplasm/nucleus elemental composition, and cell/nuclear radius (individual dose ratios are not presented but summary data appear in figures 3.2(a) and 3.2(b)). This suggests that the local cytoplasm, with its position directly adjacent to the nucleus, plays a more important role than the ECM and surrounding cells in determining nuclear dose, especially for low-energy sources. These observations are also supported by the smaller deviations from unity for $D_{n u c}^{1} / D_{n u c}$ (figure 3.2(c)) compared to those for $D_{n u c, m} / D_{n u c}$ (figure $3.2(\mathrm{a})$ ) or $D_{n u c, m} / D_{n u c}^{1}$ (figure 3.2(b)).

The dose ratios presented in figure 3.2 are sensitive to bulk tissue. For example, $D_{n u c, m} / D_{n u c}^{1}$ (dose to a single nucleus relative to nuclear dose for a single cell) is consistently less than unity for mammary gland but higher than unity for melanoma (figure 3.2(b)), i.e., modelling the cytoplasm results in a higher nuclear dose for mammary gland and lower for melanoma. Photoelectric cross sections are lower for mammary gland and higher for melanoma than other media simulated for energies below $100 \mathrm{keV}$ (see corresponding trends in mass energy absorption coefficients - figure 2.1). For example, the photoelectric cross section for cytoplasm composition model 'c' is $29 \%$ higher (10\% lower) than that of mammary gland (melanoma) at $20 \mathrm{keV}$, resulting in more (fewer) electrons set in motion in the immediate vicinity of the nucleus when the cytoplasm is present. These low-energy, short range electrons will deposit most of their energy within the nucleus, resulting in the observed trends. 
Energy deposition in the cytoplasm is sensitive to the presence of ECM and surrounding cells, as demonstrated by deviations from unity of the ratio $D_{c y t}^{1} / D_{c y t}$ (cytoplasm dose for a single cell relative to cytoplasm dose of the central cell in the cell cluster) in figure $3.2(\mathrm{~d})$. The slightly larger deviations from unity for $D_{\text {cyt }}^{1} / D_{\text {cyt }}$ compared to the corresponding nuclear dose ratio $D_{n u c}^{1} / D_{n u c}$ reflect the location of the cytoplasm at the periphery of the cell. For example, $D_{c y t}^{1} / D_{c y t}$ varies from unity by up to $11 \%$ for mammary gland with $20 \mathrm{keV}$ incident photons, which is almost twice the maximum deviation observed for $D_{n u c}^{1} / D_{n u c}$.

\subsubsection{Nuclear and cytoplasm dose variations with cell size}

Cellular dose descriptors are sensitive to variations in subcellular compartment sizes, as demonstrated by the MC simulation results for 20, 30 and $50 \mathrm{keV}$ incident photons presented in figure 3.3 for $D_{n u c} / D_{n u c, r e f}$, and figure 3.4 for $D_{c y t} / D_{c y t, r e f}$, where 'ref' refers to the reference case taken as $\left(r_{\text {cell }}, r_{n u c}\right)=(7.35,5) \mu \mathrm{m}$ (equal to the cell and nucleus sizes considered by Thomson et al. $\left.{ }^{35}\right)$. Variations in $D_{n u c} / D_{\text {nuc,ref }}$ and $D_{\text {cyt }} / D_{\text {cyt,ref }}$ demonstrate the combined effect of changing the size of the cavity in which dose is scored and the sizes of all other subcellular compartments, including those of the surrounding cells. For 90 and $370 \mathrm{keV}$ sources, dose ratios $D_{n u c} / D_{\text {nuc,ref }}$ and $D_{\text {cyt }} / D_{\text {cyt,ref }}$ deviate from unity by at most $2 \%$ (results not shown).

Variations in cell nucleus and cytoplasm doses with cell size are most pronounced for the lower-energy sources. The largest deviations are observed for mammary gland at $20 \mathrm{keV}$ with discrepancies from unity of $8 \%$ for $D_{\text {nuc }} / D_{\text {nuc,ref }}$ when $\left(r_{\text {cell }}, r_{\text {nuc }}\right)=$ $(5,4) \mu \mathrm{m}$ (figure $3.3(\mathrm{~b}))$, and $5 \%$ for $D_{\text {cyt }} / D_{\text {cyt }, \text { ref }}$ when $\left(r_{\text {cell }}, r_{n u c}\right)=(10,9) \mu \mathrm{m}$ (figure 3.4(b)). Considering cellular doses over the whole range of cell/nucleus sizes (i.e., not relative to the 'reference' case), variations are larger: $D_{n u c}\left(D_{c y t}\right)$ varies with $r_{c e l l}$ and $r_{\text {nuc }}$ by up to $13 \%$ (8\%) for gland at $20 \mathrm{keV}$. As pointed out earlier, mammary gland is an outlier in terms of radiological parameters (figure 2.1); nucleus and cytoplasm doses 

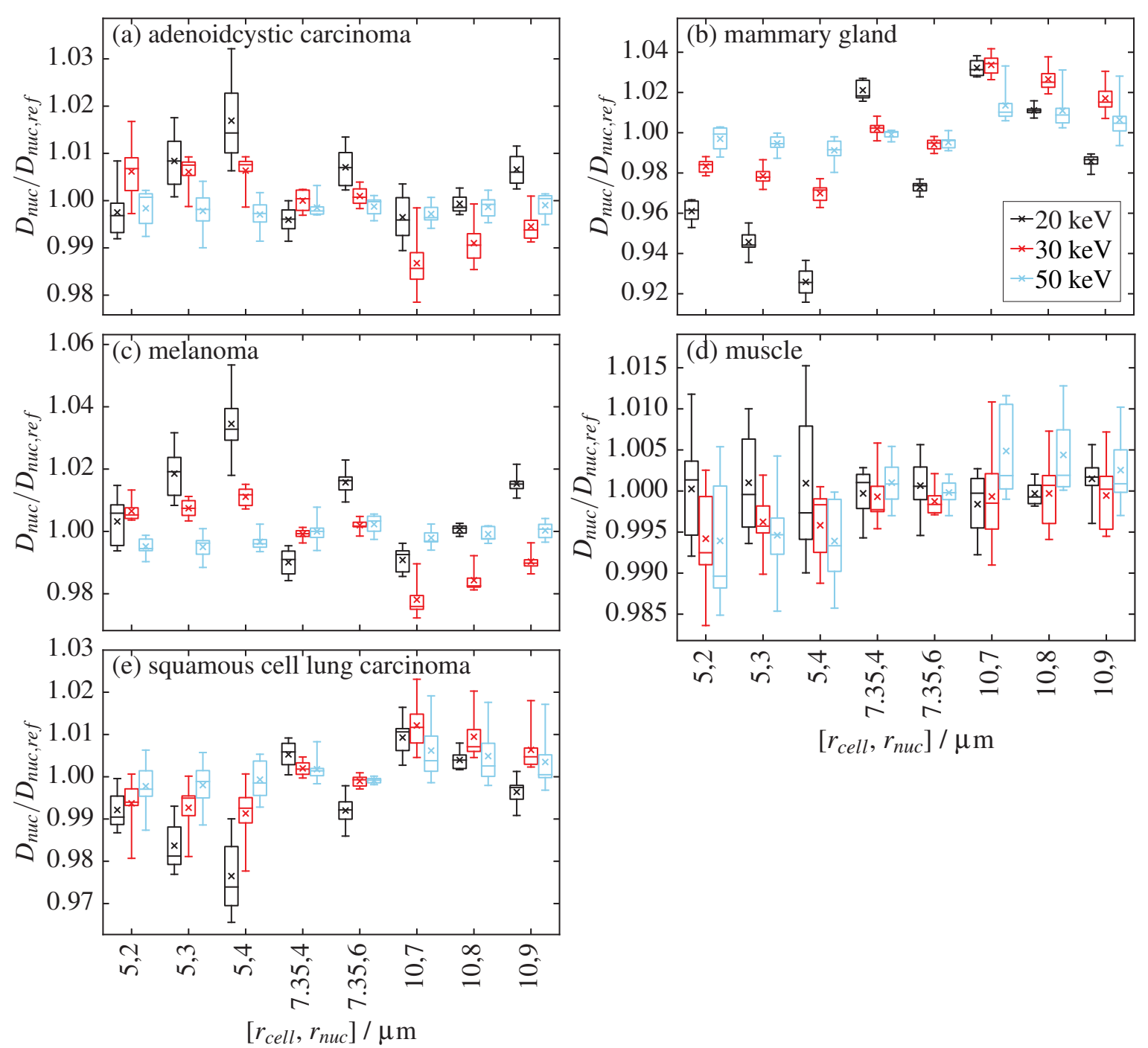

Figure 3.3: Summary of results for $D_{n u c} / D_{n u c, r e f}$, where $D_{n u c, r e f}$ is the value of $D_{n u c}$ for the reference case where $\left(r_{\text {cell }}, r_{n u c}\right)=(7.35,5) \mu \mathrm{m}$ for 20,30 and $50 \mathrm{keV}$ incident photons. Whiskers extend between the minimum and maximum values of the set of results for the seven cytoplasm/nucleus elemental compositions, box extends between the upper and lower quartiles, its horizontal line indicates the median, and the ' $x$ ' symbol indicates the mean value. 

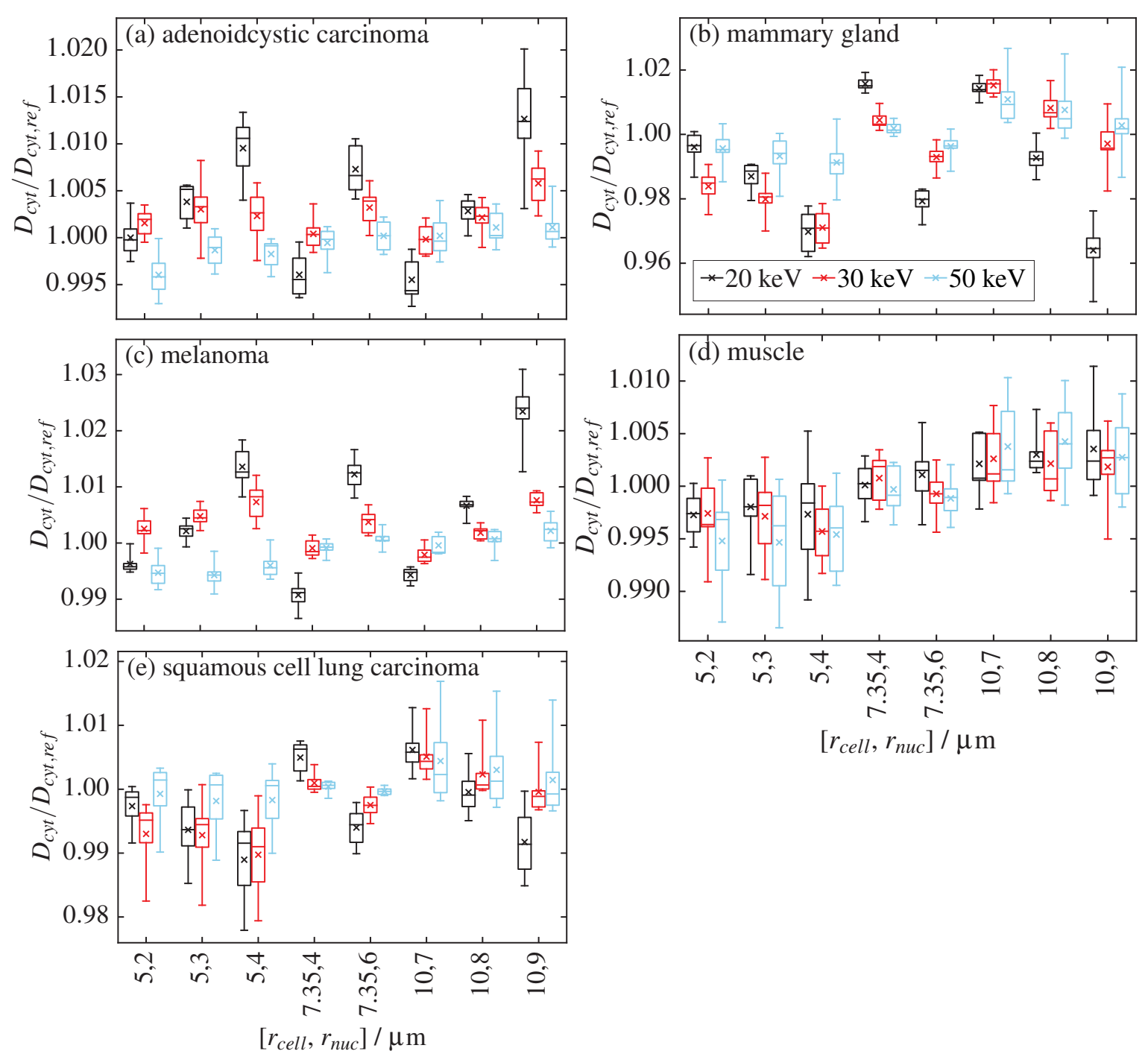

Figure 3.4: As in figure 3.3, except for $D_{\text {cyt }} / D_{\text {cyt,ref }}$, where $D_{\text {cyt,ref }}$ is the value of $D_{\text {cyt }}$ for the reference case where $\left(r_{\text {cell }}, r_{n u c}\right)=(7.35,5) \mu \mathrm{m}$ for 20,30 and $50 \mathrm{keV}$ incident photons. 
are strongly affected by size changes of subcellular compartments having media that are especially radiologically different from surroundings.

Trends in $D_{n u c} / D_{n u c, r e f}$ as a function of cell/nuclear radii reflect secondary electron ranges and media cross sections. For example, $D_{n u c} / D_{n u c, r e f}$ increases with $r_{n u c}$ for a given $r_{\text {cell }}$ (and source energy) for melanoma (figure 3.3(c)), while the trend is opposite for mammary gland (figure 3.3(b)). For fixed $r_{\text {cell }}$, increasing $r_{n u c}$ corresponds to a decreasing 'thickness' of cytoplasm and hence closer proximity of the outermost parts of the nucleus to the ECM surrounding the cell: more electrons set in motion in the ECM reach the nucleus and deposit energy therein (compared with smaller $\left.r_{n u c}\right)$. For melanoma (gland) and corresponding ECMs, interaction cross sections are relatively high (low) compared to other media (including cytoplasm and nuclear media) and hence this closer proximity to the ECM results in more (less) nuclear dose as $r_{n u c}$ increases for a given $r_{c e l l}$. The trends in $D_{c y t} / D_{\text {cyt,ref }}$ with variations in $r_{c e l l}$ and $r_{n u c}$ (figure 3.4) are qualitatively similar to the trends observed for $D_{\text {nuc }} / D_{\text {nuc, ref }}$ (figure 3.3).

\subsubsection{Comparison of macroscopic and microscopic dose de- scriptors}

Ratios of nuclear and cytoplasm doses to the corresponding homogeneous bulk tissue doses $\left(D_{m, m}\right)$ are presented in figure 3.5 for $r_{c e l l}=7.35 \mu \mathrm{m}$ and $20 \mathrm{keV}$ photons. These results demonstrate the variation in $D_{n u c}$ and $D_{c y t}$ with $r_{n u c}$ for the given $r_{c e l l}$, as well as variations among the seven cytoplasm/nucleus elemental compositions considered. The largest deviations in cellular doses from $D_{m, m}$ are observed for the smallest $r_{n u c}$ (thicker cytoplasm) and nearest unity for the largest $r_{n u c}$ (thinner cytoplasm). With a thinner cytoplasm (larger $r_{n u c}$ ), electrons contributing to nuclear dose are more likely to come from different parts of the surroundings (than for smaller $r_{n u c}$ and thicker cytoplasm) and hence there is an effective averaging over the different microscopic geometries, resulting in $D_{n u c}$ nearer $D_{m, m}$ than for smaller $r_{n u c}$. These observations are consistent 
with the perturbative effect of the cytoplasm demonstrated in figure 3.2(b). Analogous observations and reasoning exist for cytoplasm doses.
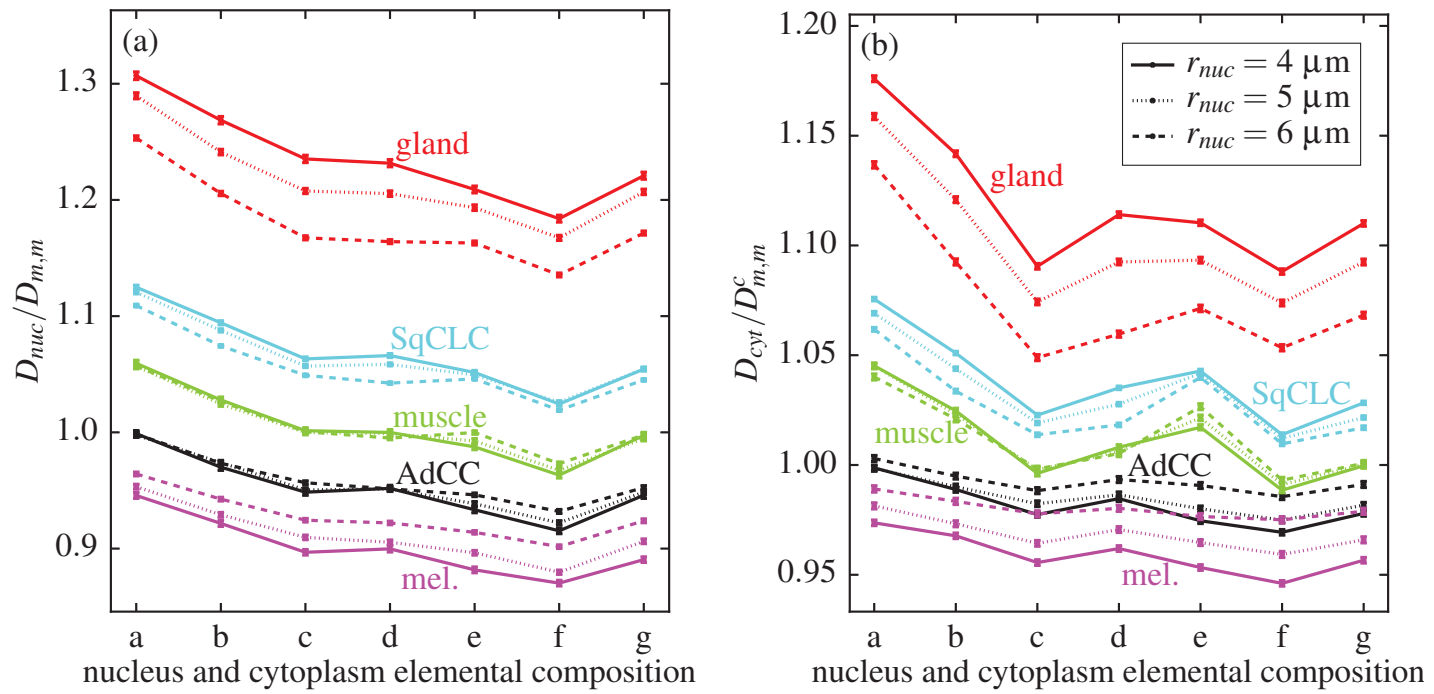

Figure 3.5: (a) $D_{n u c} / D_{m, m}$ and (b) $D_{c y t} / D_{m, m}^{c}$ for $r_{\text {cell }}=7.35 \mu \mathrm{m}$, for three nuclear radii, for $20 \mathrm{keV}$ incident photons, and for five bulk tissues: adenoidcystic carcinoma ('AdCC'), mammary gland ('gland'), melanoma ('mel'), muscle and squamous cell lung carcinoma ('SqCLC'). Although the cellular elemental compositions are independent, data points are connected with lines to guide the eye. Error bars are often too small to see.

Relations between nucleus/cytoplasm doses with macroscopic dose descriptors (doseto-medium and dose-to-water) vary with source energy, bulk tissue, cell elemental composition, and subcellular compartment size, as summarized in figures 3.6 and 3.7. The largest differences between $D_{n u c}$ and either $D_{m, m}$ or $D_{w, m}$ are observed for mammary gland with $20 \mathrm{keV}$ incident photons: $32 \%$ for $D_{n u c} / D_{m, m}$ when $\left(r_{\text {cell }}, r_{\text {nuc }}\right)=(10,7) \mu \mathrm{m}$, and $21 \%$ for $D_{n u c} / D_{w, m}$ when $\left(r_{c e l l}, r_{n u c}\right)=(7.35,4) \mu \mathrm{m}$. For the cytoplasm, the largest differences between $D_{c y t}$ and either $D_{m, m}^{c}$ or $D_{w, m}^{c}$ are observed for $\left(r_{c e l l}, r_{n u c}\right)=$ $(10,7) \mu \mathrm{m}$, for $20 \mathrm{keV}$ incident photons: $18 \%$ for $D_{c y t} / D_{m, m}^{c}$ for mammary gland, and $8 \%$ for $D_{c y t} / D_{w, m}^{c}$ for melanoma. As the source energy increases, ratios of microscopic dose descriptors relative to macroscopic dose descriptors approach unity, and there is less variation among results for different cell/nucleus sizes and elemental compositions. At $90 \mathrm{keV}$ and above, ratios of these dose descriptors converge within $3 \%$ of unity, 
although differences of up to $7 \%$ persist at $50 \mathrm{keV}$.

In general, $D_{n u c}$ matches more closely with $D_{w, m}$ than $D_{m, m}$ for all bulk tissues except for muscle. $D_{c y t}$ matches more closely with $D_{w, m}^{c}$ for mammary gland and squamous cell lung carcinoma but not adenoidcystic carcinoma, melanoma and muscle. The spread of data for $D_{n u c} / D_{m, m}$ is comparable to that of $D_{n u c} / D_{w, m}$, and the same is true for $D_{c y t} / D_{m, m}^{c}$ and $D_{c y t} / D_{w, m}^{c}$. However, the spread of data for each dose ratio in figure 3.6 (nuclear doses) is generally larger than that of figure 3.7 (cytoplasm doses).

\subsection{Discussion}

The multicellular models of various normal and cancerous human tissues used herein have demonstrated the important role played by the local cytoplasm, ECM and surrounding cells in determining the nuclear dose. Modelling these details results in differences between $D_{n u c, m}$ and $D_{n u c}$ of up to $13 \%$ at $20 \mathrm{keV}$. In some cases, a single nucleus surrounded by bulk tissue (as modelled by Enger et al..$^{55}$ ) is a suitable approximation for a multicellular model, however, this approximation loses accuracy with decreasing energy, especially for incident photon energies $<50 \mathrm{keV}$. The presence of the cytoplasm has a larger effect on the nuclear dose than the ECM and surrounding cells, i.e., $D_{n u c, m} / D_{n u c}^{1}$ generally deviates more from unity than $D_{n u c}^{1} / D_{n u c}$ (figures $3.2(\mathrm{~b})$ and $3.2(\mathrm{c}))$.

Computational constraints limited the scope of this chapter to modelling clusters of 13 cells, rather than cells spanning macroscopic volumes. To investigate sensitivity of nuclear and cytoplasm doses to cluster size, a subset of simulations was repeated with an additional layer of cells surrounding the 13-cell cluster (total of 57 cells). For mammary gland, nine cell/nucleus size combinations, seven cytoplasm/nucleus elemental compositions, and five energies were considered; for the other four bulk tissues, only $50 \mathrm{keV}$ incident photons were considered. Doses were scored in the nucleus and cyto- 

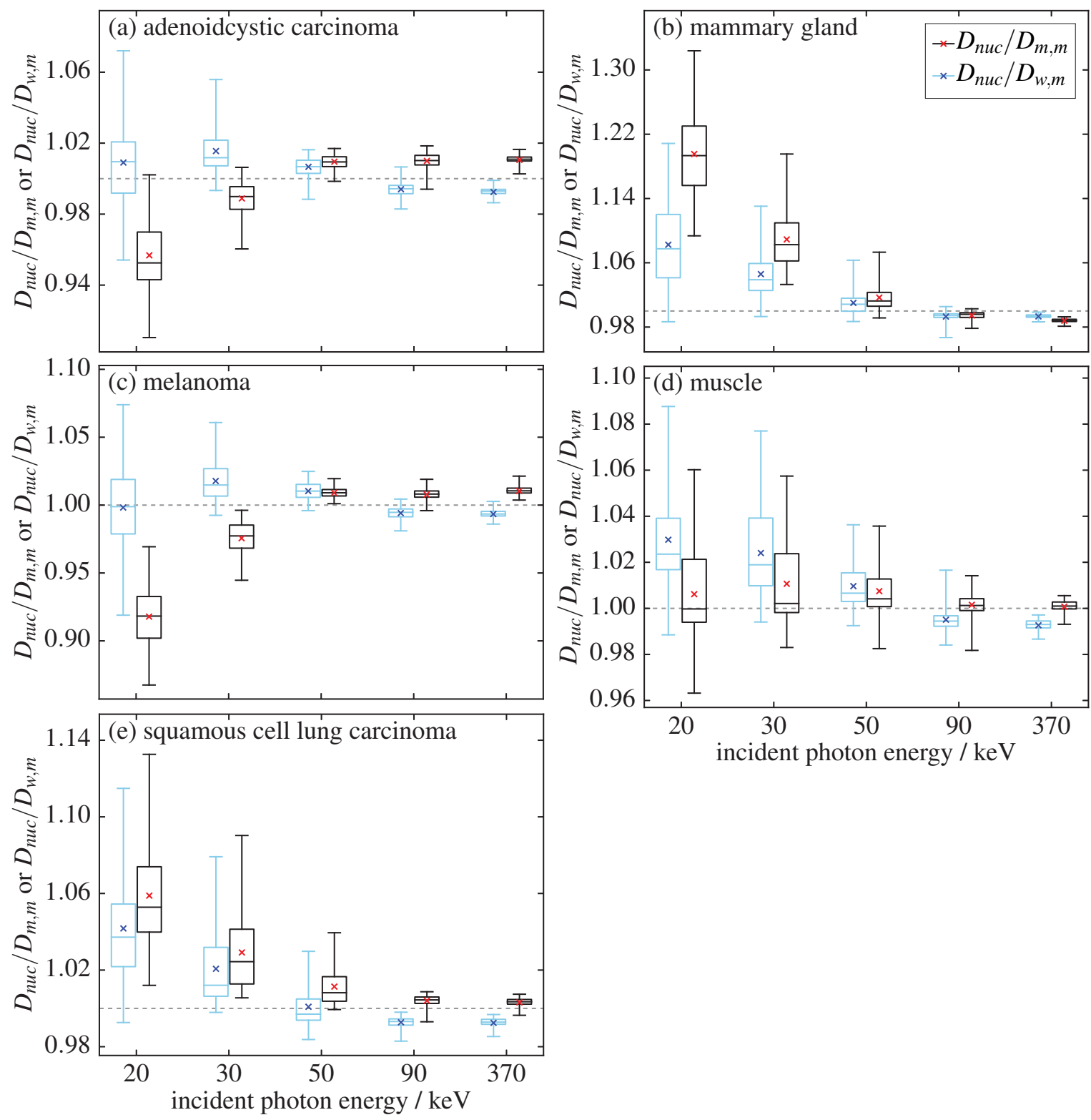

Figure 3.6: Summary of results for $D_{n u c} / D_{m, m}$ and $D_{n u c} / D_{w, m}$. See figure 3.2 for an explanation of the box and whisker plots. The horizontal dashed line indicates where the vertical axis is unity. 

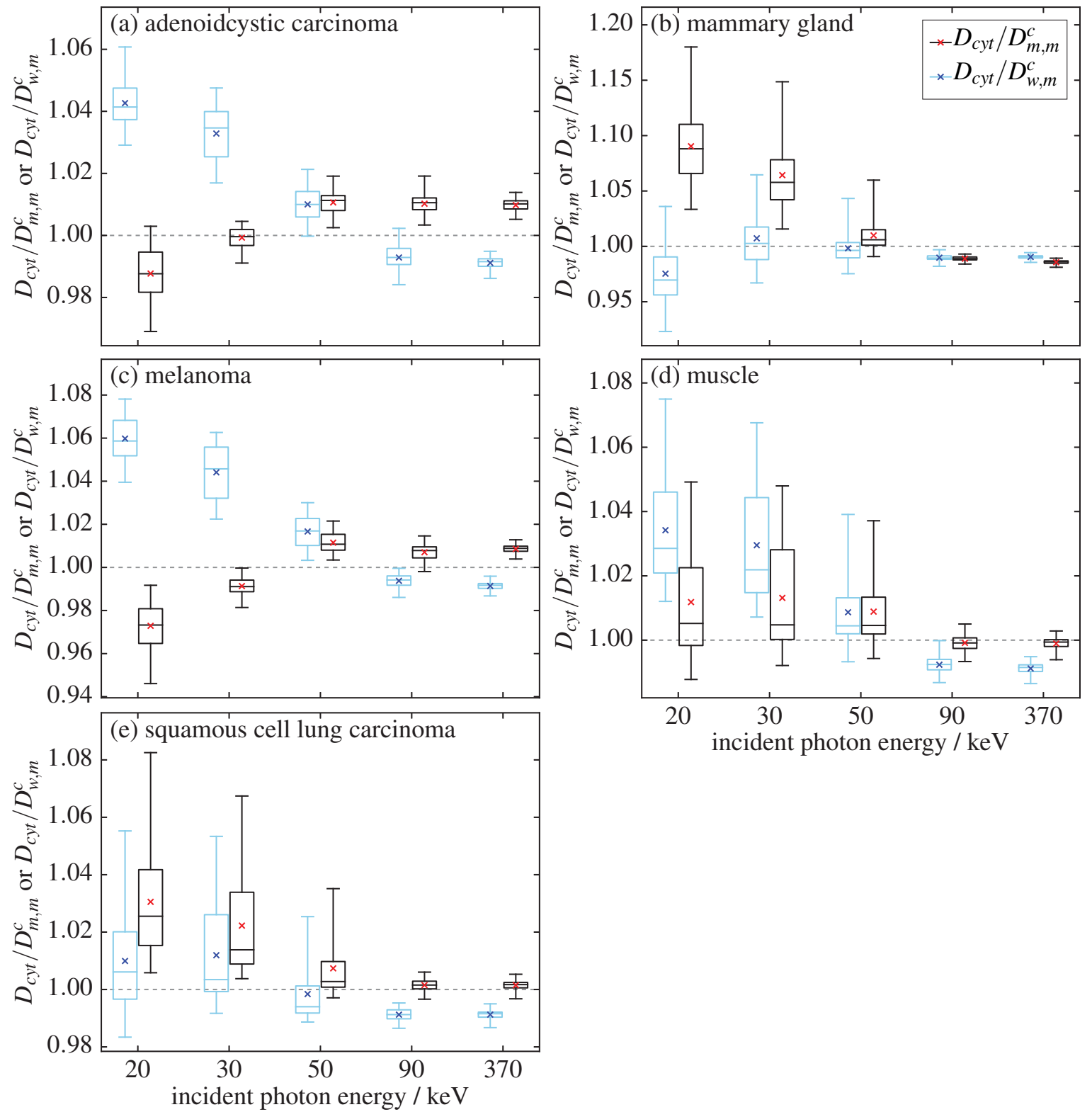

Figure 3.7: Summary of results for $D_{c y t} / D_{m, m}^{c}$ and $D_{c y t} / D_{w, m}^{c}$. See figure 3.2 for an explanation of the box and whisker plots. The horizontal dashed line indicates where the vertical axis is unity. 
plasm of the central cell, resulting in discrepancies $<2 \%$ compared to the 13 -cell model in all cases except for gland at $50 \mathrm{keV}$ (results not shown), with $1 \sigma$ uncertainties less than $0.9 \%$. For gland with $50 \mathrm{keV}$ photons incident, the maximum discrepancy was $4 \%$, for $\left(r_{\text {cell }}, r_{n u c}\right)=(7.35,4) \mu \mathrm{m}$ with nucleus/cytoplasm elemental composition 'a'. At $50 \mathrm{keV}$, there are (still) considerable variations in radiological parameters (figure 8) and $R_{C S D A}$ is larger than the radii of the 13-cell clusters. Hence, electrons depositing dose in the central nucleus may originate from the surrounding cells, ECM, or bulk tissue surrounding the ECM. Mammary gland contains a considerable lipid component $\left(31 \%\right.$ by mass $\left.^{84}\right)$, while cellular media have relatively little lipid; hence, most of the lipid is contained in the ECM so that the volume-average elemental composition of the cells and ECM approximates the bulk tissue. This results in a fluence perturbation due to the low effective atomic number of lipid. More detailed models involving adipocytes (lipid storage cells) along with glandular cells within larger clusters of cells are considered in chapter 7 . For higher energies $(\geq 90 \mathrm{keV}), R_{C S D A}$ is larger than the radii of the 13-cell clusters, and hence the lack of explicitly-modelled cells could perturb the fluence incident on the cell cluster. However, the small discrepancies between 13and 57-cell cluster results suggests that this potential issue is dominated by the convergence of radiological parameters at these energies (figure 2.1), so that the fluence is unperturbed.

Human cells exist in a range of different sizes (table 2.2). Furthermore, the relative size of the nucleus compared to the cell is larger for malignant cells than for healthy cells, ${ }^{96,97,104,105,112}$ and has been shown to correlate with patient survival in lung and breast cancer. ${ }^{113,114}$ Sizes of subcellular compartments will also depend on cell cycle stage, and vary from patient to patient. The work presented herein demonstrates that variations in subcellular compartment size result in changes in nuclear (cytoplasm) dose of up to $13 \%(8 \%)$. This sensitivity of microscopic dose descriptors to morphological differences emphasizes the importance of accurate cell models. 
El-Hussein et al. ${ }^{115}$ reported that malignant tissues have significantly more calcium and magnesium than normal tissues, based on laser-induced breakdown spectroscopy (LIBS) measurements. This increased inorganic content corresponds to enhanced photoelectric cross sections, so that more electrons are set in motion, resulting in an increase in energy deposition. For example, cell composition 'a' contains the largest mass fractions of magnesium, phosphorus, sulfur and potassium. The increased abundance of elements with higher atomic numbers results in an enhancement in $D_{n u c}$ and $D_{c y t}$ compared to the results for the other compositions (figure 3.5).

The importance of using accurate and realistic elemental compositions in cellular dosimetry simulations is emphasized in figure 3.5 , where variations in $D_{n u c}$ and $D_{\text {cyt }}$ among seven cytoplasm/nucleus elemental compositions are demonstrated. These elemental compositions are based on a literature review of normal and cancerous animal cells and represent a range of possible water, lipid, organic and inorganic content. ${ }^{35}$ There is a need for accurate cellular elemental composition data, particularly for human cells. While LIBS has demonstrated promise in characterizing tissues as normal or malignant, ${ }^{115}$ it has not been used to produce absolute measurements of elemental composition within individual cell compartments. Some cellular dosimetry studies rely on inaccurate data or unrealistic assumptions which will potentially have a significant effect on results and conclusions. For example, some of the cellular elemental compositions used by Incerti et al., ${ }^{53}$ Barberet et $a l .{ }^{54}$ and Enger et $a l .{ }^{55}$ do not fall within the range of typical values. ${ }^{35}$ Further, the cytoplasm elemental composition of Byrne et al. ${ }^{56,116}$ determined by subtracting nucleus elemental mass fractions ${ }^{85}$ from the average of male and female soft tissue compositions, ${ }^{117}$ has an unrealistically high carbon content compared to the cells of most soft tissues due to the considerable fatty content of the soft tissue composition used. Other authors use only water in their simulations ${ }^{64,118,119}$ despite the fact that inter- and intra-cellular media are not radiologically equivalent to water. Results are also strongly influenced by the choice of bulk tissue. Bulk tissue 
compositions are often based on a small sample size that is not necessarily representative of the population (e.g., Maughan et al. ${ }^{86}$ use up to three biopsy specimens to determine various malignant tissue compositions, and Woodard and White ${ }^{84}$ use seven specimens from post-menopausal women to determine mammary gland composition).

Relations between microscopic dose descriptors (nuclear and cytoplasm doses) and macroscopic dose descriptors (dose-to-water and dose-to-medium) are sensitive to source energy, tissue type, as well as cell/nucleus size and composition. Cavity theory (see chapter 4) can be applied to the MC results presented herein to further understand relationships between macro- and microscopic doses, however, this is beyond the scope of the current chapter. Comparison of nuclear and cytoplasm doses with macroscopic dose descriptors suggests that under certain conditions, dose-to-water and/or dose-tomedium are suitable surrogates for cellular dose descriptors. However, their suitability decreases with energy; there are many cases below $50 \mathrm{keV}$ where there is considerable disagreement between macro- and microscopic dose descriptors (figures 3.6 and 3.7). For all bulk tissues except for muscle, the mean/median for $D_{n u c} / D_{w, m}$ is closer to unity than for $D_{\text {nuc }} / D_{m, m}$; however, there is considerable variability in $D_{\text {nuc }} / D_{w, m}$, with discrepancies up to $21 \%$ observed at $20 \mathrm{keV}$. Given the wide variety of cell morphologies and elemental compositions present in the human body, it is improbable that a universal surrogate dose descriptor for cellular doses exists. In terms of the debate over the medium for dose specification for model-based dose calculation algorithms, ${ }^{31,32}$ our results do not provide particular support for either $D_{w, m}$ or $D_{m, m}$.

In this chapter, monoenergetic sources and a single cell cluster depth of $1 \mathrm{~cm}$ in phantom are considered. For realistic spectral sources, monoenergetic results can be combined with suitable weighting factors to obtain a dose estimate. Additional simulations were carried out to compare spectral and monoenergetic sources (results not shown): a mammography spectrum (mean energy of $16.8 \mathrm{keV})^{33}$ with mammary gland as the bulk tissue; spectrum emitted from the Amersham OncoSeed $6711{ }^{125} \mathrm{I}$ 
brachytherapy source (mean energy of $27.3 \mathrm{keV})^{81}$ with melanoma or muscle as the bulk tissue. Simulations were carried out for all seven cytoplasm/nucleus elemental compositions and nine cell/nucleus size combinations. Simulating the full spectrum instead of using the mean energy of the spectrum (i.e., a monoenergetic source) resulted in differences in each of $D_{n u c} / D_{m, m}$ and $D_{n u c} / D_{w, m}$ of up to $0.74 \%$; however, absolute doses (e.g., $D_{n u c}, D_{m, m}$, etc.) varied considerably. To investigate the effect of changing the depth of the cell cluster within the bulk tissue phantom, simulations were also carried out for cell clusters located at the centres of phantoms with side lengths of $1 \mathrm{~cm}$ and $4 \mathrm{~cm}$. Using the mammography source spectrum, this resulted in differences in $D_{n u c} / D_{m, m}$ and $D_{n u c} / D_{w, m}$ of up to $1.2 \%$ compared to the usual $2 \mathrm{~cm}$ case. For increasing depths, beam hardening occurs, which will be an especially large effect for spectra containing a large proportion of low energy photons with relatively short mean free paths.

\subsection{Conclusions}

Our results demonstrate that microscopic dose descriptors vary with subcellular compartment size, elemental composition and source energy. The surrounding geometry (the presence of a local cytoplasm, ECM or other cells) has a considerable influence on the nuclear dose and is important to consider given the significance of the nucleus as a target for radiation-induced cell death. The variation in nuclear and cytoplasm doses with respect to the details of the microscopic tissue structure model demonstrates the importance of accurate knowledge of cellular properties. Given the differences in morphology and composition between normal and cancerous cells, the computational models used in this chapter are relevant for investigating differences in energy deposition and biological response between healthy and cancerous cells. In the context of the debate regarding the dose specification medium for MC treatment planning, with the nucleus and cytoplasm as biologically relevant targets, neither dose-to-water nor 
dose-to-medium quantitatively tracks energy deposition within these cellular targets in a reliable manner, although agreement within $3 \%$ is observed for energies $\geq 90 \mathrm{keV}$. Differences between dose descriptors are especially pronounced for low incident photon energies $(\leq 50 \mathrm{keV})$, where radiological parameters are strongly medium-dependent and the ranges of electrons are comparable to cellular length scales, so that energy deposition is sensitive to model details. 


\section{Chapter 4}

\section{Cavity theory applications for kilovoltage cel- lular dosimetry}

Cavity theory is introduced as a method for relating macroscopic and microscopic dose descriptors. A survey of small, intermediate and large cavity theory approaches is presented. Different cavity theory approaches are evaluated via comparison with Monte Carlo results for a range of kilovoltage photon energies. Based on the suggestion that clusters of water molecules associated with DNA are important radiobiological targets, doses to small volumes of water within the nucleus are estimated using small cavity theory. 


\subsection{Introduction}

Despite considerable discussion in the literature regarding applications of cavity theory to predict doses to cellular compartments (see chapter 1), no such cavity theory predictions have been compared with results of MC simulations involving detailed, multicellular microscopic tissue models - this is the focus of this chapter. We investigate

relationships between macroscopic (bulk tissue) and microscopic (cellular) dose descriptors using MC simulations and cavity theory, focusing on kilovoltage photon energies. Various cavity theory approaches are evaluated by comparing SCT, LCT, and multiple ICT predictions of $D_{w, m} / D_{m, m}$ with corresponding $\mathrm{MC}$ results for a range of source energies, bulk tissue media and several $\sim \mu$ m-sized (i.e., nucleus-sized) cavities (part I). The cavity theory approach providing the closest overall agreement with MC results for $D_{w, m} / D_{m, m}$ is then used to predict nuclear doses which are compared with MC simulation results from detailed, multicellular models of healthy and cancerous human soft tissues, considering a range of representative subcellular compartment sizes and elemental compositions (part II). Finally, SCT conversion factors applied to MC simulation results are investigated for prediction of energy deposition within a small volume of water in nucleus (part III).

\subsection{Methods}

Section 4.2.1 provides an overview of the three parts of the cavity theory study covered in this chapter, before details on MC simulations used to determine fluence spectra and cavity theory methods are given in sections 4.2 .2 and 4.2 .3 , respectively. 


\subsubsection{Overview}

The three parts of our study are schematically depicted in figure 4.1 and described further below. In each case, the source is a parallel beam of monoenergetic photons with energies of $20,30,50,90$ or $370 \mathrm{keV}$, covering the range of photon energies of common brachytherapy sources, as well as diagnostic radiology. The ranges of electrons set into motion in soft tissues by these kilovoltage photons are comparable to sizes of subcellular compartments, as discussed in the previous chapter. The beam has a circular cross section with $1 \mathrm{~cm}$ radius. Five bulk tissues are considered: adenoidcystic carcinoma, mammary gland, melanoma, muscle and squamous cell lung carcinoma.

In part I, values of $D_{w, m} / D_{m, m}$ (figure 4.1(a)) from MC simulations (section 4.2.2) are compared with corresponding cavity theory predictions, considering five ICT approaches, plus SCT and LCT (section 4.2.3). Three cavity radii, five bulk tissues and five incident photon energies are considered, corresponding to a total of $N=75$ scenarios. The cavity theory method with the lowest root mean square error (RMSE) when compared with MC results is used in part II of the study presented in this chapter. The RMSE is calculated as follows:

$$
\mathrm{RMSE}=\sqrt{\frac{1}{N}\left[\sum_{i=1}^{N}\left(\left(\frac{D_{w, m}}{D_{m, m}}\right)_{i, C T}-\left(\frac{D_{w, m}}{D_{m, m}}\right)_{i, M C}\right)^{2}\right]}
$$

where the sum is over the entire parameter space considered (i.e., $N=75$ ), and the quantities $\left(D_{w, m} / D_{m, m}\right)_{i, C T}$ and $\left(D_{w, m} / D_{m, m}\right)_{i, M C}$ are the values (for scenario $i$ ) of $D_{w, m} / D_{m, m}$ according to cavity theory and MC simulation, respectively.

Nuclear doses $\left(D_{n u c}\right)$ are considered in part II (figure 4.1(b)). MC simulation results for $D_{n u c} / D_{m, m}$ are compared with corresponding cavity theory predictions (using the method which minimizes the RMSE from part I). MC simulations for $D_{n u c}$ involve detailed, multicellular models of normal and cancerous human soft tissues, described in 
Part I: Evaluation of cavity theory approaches

(a) one cavity

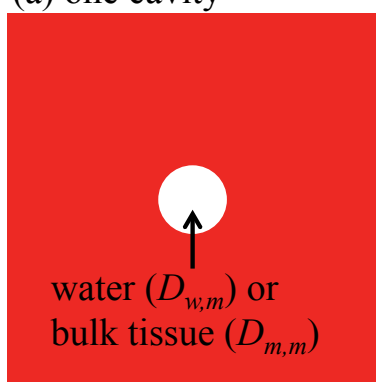

$r_{c a v}=3,6,9 \mu \mathrm{m}$

Part II: Cavity theory applied to nuclear $\operatorname{doses}\left(D_{n u c} \& D_{n u c, m}\right)$

(b) cell cluster

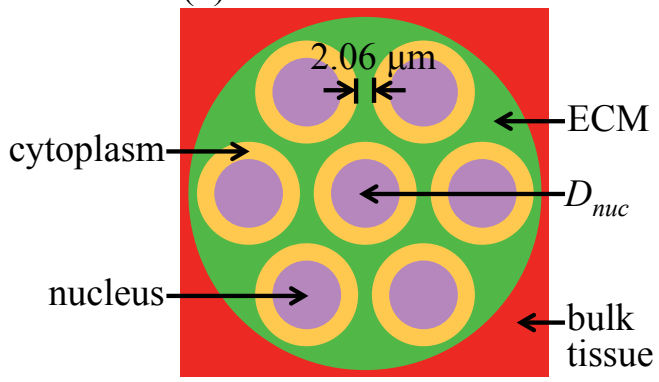

(c) single nucleus

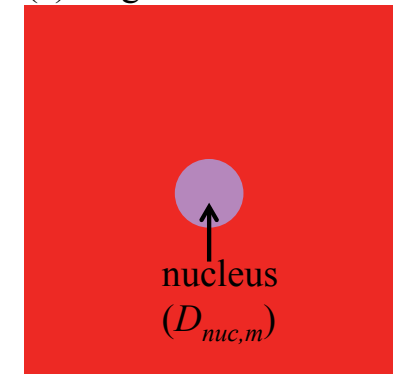

\begin{tabular}{|l|l|l|l|}
\hline$r_{\text {cell }} / \mu \mathrm{m}$ & 5 & 7.35 & 10 \\
\hline$r_{n u c} / \mu \mathrm{m}$ & $2,3,4$ & $4,5,6$ & $7,8,9$ \\
\hline
\end{tabular}

Part III: Using Bragg-Gray SCT to estimate dose to small water cavities

(d) small water cavity

(e) small water cavity in cell cluster
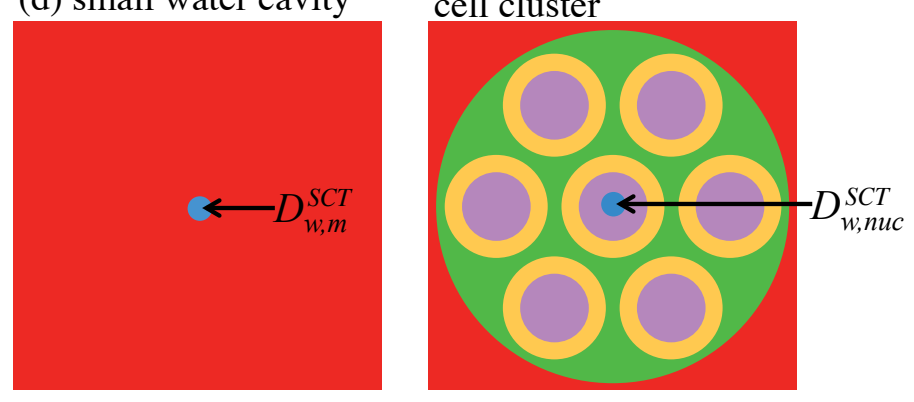

Figure 4.1: Schematic diagrams summarizing the three parts of the cavity theory study. Part I: (a) a single cavity (radius $=r_{\text {cav }}$ ) consisting of either water or bulk tissue is embedded at the centre of a bulk tissue phantom. Part II: nuclear doses are considered. (b) Cross section of multicellular model consisting of 13 cells in a hexagonal lattice with cells $\left(\right.$ radius $=r_{\text {cell }}$ ) embedded in a sphere of extracellular matrix $(\mathrm{ECM})$ (radius 18 to $33 \mu \mathrm{m}$ ); dose is scored in the central nucleus (radius $=r_{n u c}$ ). (c) A single nucleus embedded in an otherwise homogeneous bulk tissue phantom. Part III: Bragg-Gray SCT is used to estimate dose to a small water cavity embedded in (d) a bulk tissue phantom, or (e) the central nucleus of a cluster of 13 cells. Each geometry is located at the centre of a $(2 \mathrm{~cm})^{3}$ cubic bulk tissue phantom. 
detail in the previous chapter and illustrated in figure 4.1(b) (see section 4.2.2). Three cell radii $r_{\text {cell }}$ are investigated, along with three nuclear radii $r_{n u c}$ for each $r_{c e l l}$; these radii are based on typical values for human cells. Additionally, seven cytoplasm/nucleus elemental compositions, five bulk tissues and five source energies are considered, for a total of 1575 scenarios. Cavity theory predictions are also compared with $D_{n u c, m} / D_{m, m}$, where $D_{n u c, m}$ is the dose to a single nucleus embedded in an otherwise homogeneous bulk tissue phantom (figure 4.1(c)).

Finally, part III considers the potential use of SCT for conversion of MC-calculated $D_{m, m}$ to $D_{w, m}$, motivated by the radiobiological importance and universality (i.e., lack of dependence on cell type) of DNA-bound water molecules within the nucleus as a radiation target. ${ }^{37,63}$ We employ SCT to estimate dose to a small volume of water in two different ways, with scenarios illustrated in figures 4.1(d) and 4.1(e). The small water cavities are not explicitly modelled in MC simulations: $D_{m, m}$ (figure 4.1(a)) and $D_{n u c}$ (figure $4.1(\mathrm{~b})$ ) are obtained from MC simulations, and SCT conversion factors (stopping power ratios - details provided in section 4.2.3) are used to estimate $D_{w, m}^{S C T}$ and $D_{w, n u c}^{S C T}$, respectively, which we compare via the ratio:

$$
\frac{D_{w, n u c}^{S C T}}{D_{w, m}^{S C T}}=\left[D_{n u c} \times\left(\frac{\overline{S_{c o l}}}{\rho}\right)_{n u c}^{w}\right] /\left[D_{m, m} \times\left(\frac{\overline{S_{c o l}}}{\rho}\right)_{m}^{w}\right]=\frac{D_{n u c}}{D_{m, m}} \times\left(\frac{\overline{S_{c o l}}}{\rho}\right)_{n u c}^{m}
$$

The elemental compositions of the five bulk tissues, seven nuclear media and seven cytoplasm media considered in this chapter are presented in table 2.1 of chapter 2 . ECM definitions are determined by requiring that the average, mass-weighted composition and density of the cells and ECM are approximately equal to those of the surrounding bulk tissue; this calculation is carried out for each bulk tissue, cell/nucleus size combination and cytoplasm/nucleus elemental composition. 


\subsubsection{Monte Carlo simulations}

MC results for $D_{n u c} / D_{m, m}$ and $D_{n u c, m} / D_{m, m}$ are from the previous chapter, while additional MC simulations for $D_{w, m} / D_{m, m}$ are carried out for the present work using the EGSnrc user-code egs_chamber. A sufficient number of histories are simulated to obtain statistical uncertainties on doses of $<0.91 \%$.

Electron and photon fluence spectra are used for calculating the spectrum-averaged mass collisional stopping power and mass energy absorption coefficient ratios needed for cavity theory estimates (section 4.2.3), and are obtained from the EGSnrc user-code

FLURZnrc. ${ }^{74}$ The geometry consists of a cylindrical phantom composed of water or bulk tissue of $2 \mathrm{~cm}$ height and diameter. The scoring region is also cylindrical (with height and diameter equal to $0.05 \mathrm{~cm}$ ) and is located at the centre of this phantom. The fluence spectra are discrete, having $1 \mathrm{keV}$ bin widths, and a minimum energy of $1 \mathrm{keV}$. Fluence spectra were found to be robust under variations in scoring region size. Electron fluence spectra are presented in figure 4.2 .

\subsubsection{Large, small and intermediate cavity theories}

The three categories of cavity theory (large, small and intermediate) and their implementations are described in the following subsections, assuming a monoenergetic photon source.

\section{Large cavity theory}

Large cavity theory (LCT) requires that the cavity is large compared to the ranges of primary electrons, yet small enough not to disturb the photon fluence in the surrounding medium. Charged Particle Equilibrium is required. In this case, the dose-to-cavity-in- 


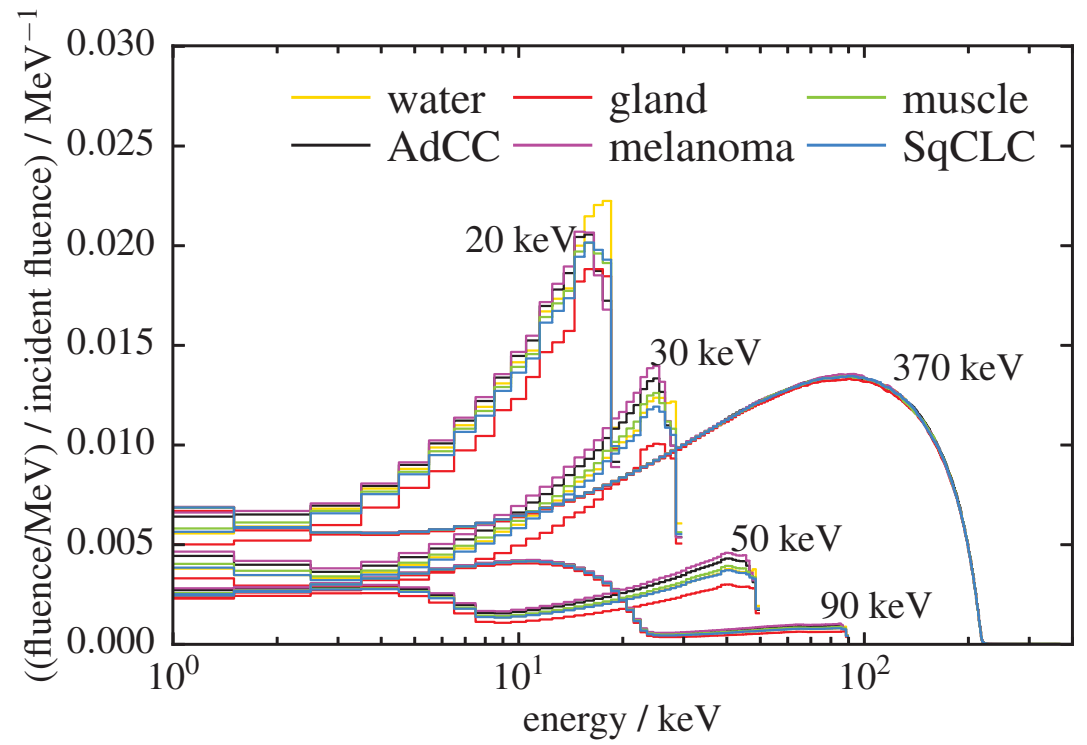

Figure 4.2: Electron fluence spectra at $1 \mathrm{~cm}$ depth within bulk tissue and water phantoms (see section 4.2.2). Results are presented for five incident photon energies, as indicated next to the corresponding spectra. All generations of electrons are included. Error bars are often too small to see. The low (high) energy peak in each spectra is due to Compton electrons (photoelectrons). The Compton (photoelectric) contribution to the spectra increases (decreases) with incident photon energy. 
medium to dose-to-medium-in-medium conversion factor is given by Nahum: ${ }^{46}$

$$
\frac{D_{c a v, m}}{D_{m, m}}=\left(\frac{\overline{\mu_{e n}}}{\rho}\right)_{m}^{c a v}=\frac{\int_{0}^{E_{\max }} E\left[\frac{\mu_{e n}(E)}{\rho}\right]_{c a v}\left[\Phi_{E}(E)\right]_{m} d E}{\int_{0}^{E_{\max }} E\left[\frac{\mu_{e n}(E)}{\rho}\right]_{m}\left[\Phi_{E}(E)\right]_{m} d E},
$$

where $D_{c a v, m}$ is the dose to a cavity embedded in a medium $m, D_{m, m}$ is the dose to the medium $m$ at the location of the cavity if the cavity did not exist, $E_{\text {max }}$ is the maximum energy in the photon spectrum, and $\mu_{e n}(E) / \rho$ is the mass energy absorption coefficient evaluated at energy $E$. The photon number fluence in the medium, differential in energy $E$ is represented by $\left[\Phi_{E}(E)\right]_{m}$, and is assumed to be the same as the fluence in the cavity $\left[\Phi_{E}(E)\right]_{c a v}$. The horizontal line above $\left(\frac{\overline{\mu_{e n}}}{\rho}\right)_{m}^{c a v}$ indicates that it is a spectrum-averaged quantity. As a consequence of using FLURZnrc fluence spectra, lower integration bounds in equation $(4.3)$ are $1 \mathrm{keV}$ in practice. Values of $\left(\frac{\overline{\mu_{e n}}}{\rho}\right)_{m}^{c a v}$ are robust to variations in the maximum fractional energy loss per step (ESTEPE) selected for FLURZnrc calculations. FLURZnrc provides fluence spectrum values at the centre of each energy bin; $\mu_{e n} / \rho$ values are also obtained at these energies, using EGSnrc usercode $\mathrm{g}^{28}$ with statistical uncertainties $<0.02 \%$. An in-house script is used to perform the numerical integration needed to evaluate the integrals in equation (4.3). This script was validated by comparison with the same calculation carried out in a spreadsheet as well as with the independent results of Carlsson Tedgren and Alm Carlsson. ${ }^{63}$ Spectrum averaged $\mu_{e n} / \rho$ ratios for water to medium are presented in figure $4.3(\mathrm{a})$.

\section{Small cavity theory}

Small cavity theory (SCT) requires that the cavity is small compared to the ranges of primary electrons. It is assumed that the presence of the cavity does not perturb the fluence of primary electrons in the surrounding medium, and that $\delta$-ray equilibrium 

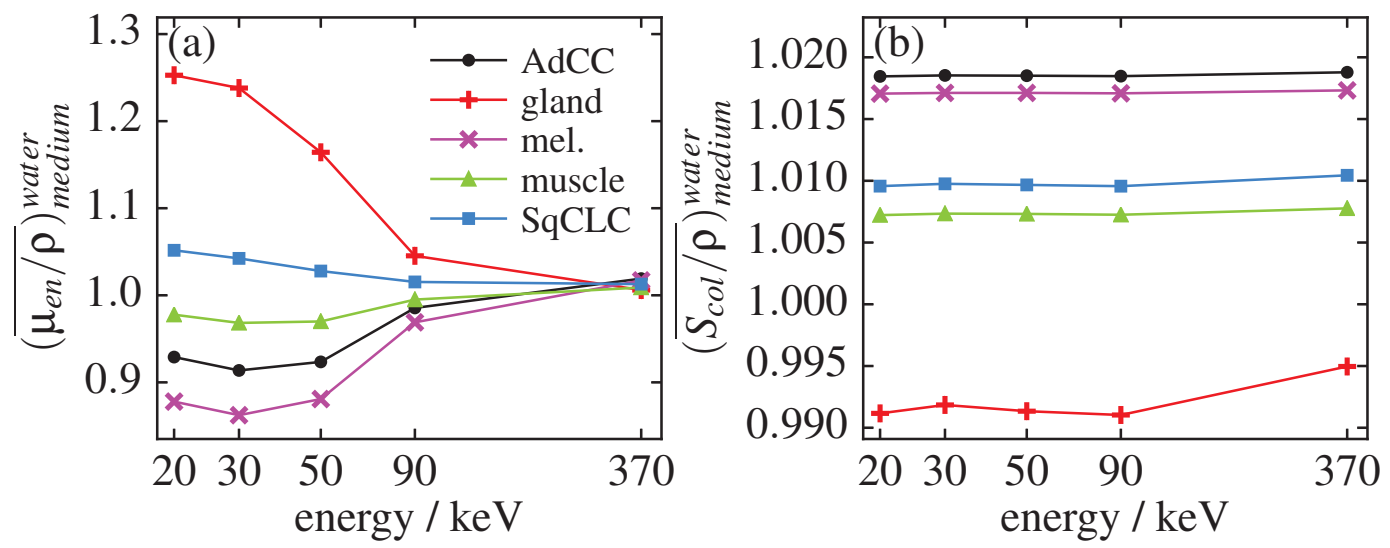

Figure 4.3: Spectrum averaged (a) mass energy absorption coefficient $\left(\mu_{e n} / \rho\right)$ and (b) mass collisional stopping power $\left(S_{c o l} / \rho\right)$ ratios for water over medium (as indicated in legend: 'AdCC' — adenoidcystic carcinoma, 'gland' — mammary gland, 'SqCLC' — squamous cell lung carcinoma). Fluence spectra correspond to a depth of $1 \mathrm{~cm}$ within a phantom of that particular medium.

exists. The conversion factor according to Bragg-Gray SCT is given by Nahum: ${ }^{46}$

$$
\frac{D_{c a v, m}}{D_{m, m}}=\left(\frac{\overline{S_{c o l}}}{\rho}\right)_{m}^{c a v}=\frac{\int_{0}^{E_{\max }}\left[\Phi_{E}^{e^{-}}(E)\right]_{m}\left[\frac{S_{c o l}(E)}{\rho}\right]_{c a v} d E}{\int_{0}^{E_{\max }}\left[\Phi_{E}^{e^{-}}(E)\right]_{m}\left[\frac{S_{c o l}(E)}{\rho}\right]_{m} d E}
$$

where $S_{c o l}(E) / \rho$ is the unrestricted mass collisional stopping power evaluated at energy $E$, and $\left[\Phi_{E}^{e^{-}}(E)\right]_{m}$ is the primary electron number fluence in the medium, differential in energy $E$ (assumed to be the same as the fluence in the cavity $\left.\left[\Phi_{E}^{e^{-}}(E)\right]_{\text {cav }}\right)$. Unrestricted mass collisional stopping powers $S_{c o l} / \rho$ used for Bragg-Gray SCT are obtained from the NIST ESTAR database, ${ }^{76}$ where uncertainties are $1 \%$ to $2 \%$ for electron energies $>100 \mathrm{keV}$; uncertainties are $2 \%$ to $3 \%$ and $5 \%$ to $10 \%$ in low- and high-Z materials, respectively, for energies between $100 \mathrm{keV}$ and $10 \mathrm{keV}$. Due to the absence of shell corrections (necessary when the incident electron velocity is comparable to that of the bound electrons), uncertainties are $\sim 10 \%$ for low-Z materials at $1 \mathrm{keV}$. Again, lower integration bounds in equation (4.4) are $1 \mathrm{keV}$ in practice. Values of $\left(\frac{\overline{S_{c o l}}}{\rho}\right)_{m}^{c a v}$ are insensitive to variations in ESTEPE used in FLURZnrc calculations. The in-house script used to evaluate equation (4.4) was validated by comparison with the same calculation carried 
out in a spreadsheet, by comparison with Carlsson Tedgren and Alm Carlsson, ${ }^{63}$ and by comparison with the SPRRZBGnrc user-code. ${ }^{120}$ Spectrum averaged $S_{\text {col }} / \rho$ ratios for water to medium are presented in figure 4.3(b).

The Spencer-Attix modification of the Bragg-Gray expression uses the restricted instead of the unrestricted stopping power, and only requires $\delta$-ray equilibrium for energies below the threshold $\Delta$, but requires that the cavity does not affect the fluence of all electrons with energies greater than $\Delta:^{121}$

$$
\begin{aligned}
& \frac{D_{\text {cav }, m}}{D_{m, m}}=\left(\overline{L_{\Delta}}\right)_{m}^{c a v} \\
& =\frac{\left\{\int_{\Delta}^{E_{\max }}\left[\Phi_{E}^{t o t, e^{-}}(E)\right]_{m}\left[\frac{L_{\Delta}(E)}{\rho}\right]_{c a v} d E\right\}+\left\{\left[\Phi_{E}^{t o t, e^{-}}(\Delta)\right]_{m} \cdot\left[S_{c o l}(\Delta) / \rho\right]_{c a v} \cdot \Delta\right\}}{\left\{\int_{\Delta}^{E_{\max }}\left[\Phi_{E}^{\text {tot, } e^{-}}(E)\right]_{m}\left[\frac{L_{\Delta}(E)}{\rho}\right]_{m} d E\right\}+\left\{\left[\Phi_{E}^{t o t, e^{-}}(\Delta)\right]_{m} \cdot\left[S_{\text {col }}(\Delta) / \rho\right]_{m} \cdot \Delta\right\}}
\end{aligned}
$$

where $\left[\Phi_{E}^{t o t, e^{-}}(E)\right]_{m}$ is the total electron number fluence in the medium, differential in energy $E$, which takes into account all generations of electrons with energy $\geq \Delta$ (i.e., including $\delta$-rays); $L_{\Delta}(E) / \rho$ is the restricted mass collisional stopping power for energy losses less than the cutoff $\Delta .{ }^{46}$ Again, it is assumed that $\left[\Phi_{E}^{\text {tot, } e^{-}}(E)\right]_{m}$ is equal to $\left[\Phi_{E}^{t o t, e^{-}}(E)\right]_{\text {cav }}$. The first terms in the numerator and denominator of equation (4.5) account for energy deposition $<\Delta$ due to electrons with $E>\Delta$; the second terms (track-end terms) account for energy deposition by electrons with $E \leq \Delta$. Following the method of Spencer and Attix, ${ }^{121} \Delta$ is taken to be the energy of an electron that is capable of just crossing the cavity (according to the mean chord length), using $R_{C S D A}$ values for electrons in water. The mean chord length is given by $L=4 \mathrm{~V} / \mathrm{S}$ (for a convex cavity), for volume $V$ and surface area $S .{ }^{122}$

Restricted stopping-power ratios are obtained from the EGSnrc user-code SPRRZnrc, ${ }^{74}$ using the same simulation geometry as in the fluence spectrum calculations (again, results were found to be independent of changes in scoring cavity size). Restricted stopping-power ratios generated by SPRRZnrc are robust to variations in ESTEPE. 
Unrestricted and restricted stopping-power ratios differ by at most $0.4 \%$ over the range of media, energies and cavity sizes considered herein. This relatively good agreement can be interpreted as evidence that $\delta$-ray equilibrium exists, and thus Bragg-Gray SCT is used throughout this chapter instead of Spencer-Attix SCT (which requires a separate MC simulation for each energy, medium and $\Delta$, where $\Delta$ is cavity size-dependent).

\section{Intermediate cavity theory}

Intermediate cavity theory (ICT) describes the case where electron ranges are comparable to the size of the cavity. The dose ratio $D_{c a v, m} / D_{m, m}$ is the sum of SCT and LCT contributions, weighted by a parameter $d:{ }^{46,123}$

$$
\frac{D_{c a v, m}}{D_{m, m}}=d \cdot\left(\frac{\overline{S_{c o l}}}{\rho}\right)_{m}^{c a v}+(1-d) \cdot\left(\frac{\overline{\mu_{e n}}}{\rho}\right)_{m}^{c a v},
$$

where $d$ varies between 0 and 1 as a function of cavity size, elemental composition and density, as well as with source energy. For certain ICT approaches, cavity surroundings also influence $d$.

We consider five approaches that have been presented in the literature for determining $d$, summarized in table 4.1 along with convenient labels. For several of the methods considered, $d$ is calculated according to: ${ }^{123}$

$$
d=\left(1-e^{-\beta L}\right) /(\beta L)
$$

where $L=4 V / S$ is the mean chord length of the cavity. Equation (4.7) is equivalent to the average attenuation factor of electrons generated in the wall as they traverse the cavity. ${ }^{123}$ The fluence of electrons generated within the cavity is assumed to build up exponentially according to the same coefficient $\beta$ that defines the exponential attenuation of wall electrons, corresponding to a build-up factor of $1-d$. Therefore, the first (second) term in equation (4.6) represents the contribution to the cavity dose from 
electrons originating in the wall (in the cavity).

Table 4.1: Summary of intermediate cavity theory approaches implemented in this chapter, with convenient labels.

\begin{tabular}{|c|c|}
\hline Label & Brief description and reference \\
\hline Burlin-Chan & $\begin{array}{l}\text { Equation (4.7) gives } d \text { with } \beta \text { given by } e^{-\beta R_{C S D A}}=0.01 \text {, using } R_{C S D A} \text { for } \\
\text { electrons of the incident photon energy. }{ }^{124}\end{array}$ \\
\hline $\begin{array}{l}\text { Burlin- } \\
\text { Janssens }\end{array}$ & $\begin{array}{l}\text { Equation (4.7) gives } d \text { with } \beta \text { given by } e^{-\beta R_{C S D A}}=0.04 \text {, using } R_{C S D A} \text { for } \\
\text { electrons of the incident photon energy. }{ }^{125}\end{array}$ \\
\hline Loevinger & $\begin{array}{l}\text { Equation }(4.7) \text { gives } d \text { with } \beta\left(\text { in } \mathrm{cm}^{-1}\right) \text { given by } \beta=\left(16 \rho_{\text {cav }}\right) /\left(T_{\max }-\right. \\
0.036)^{1.4} \text { where } \rho_{\text {cav }} \text { is the density of the cavity in } \mathrm{g} / \mathrm{cm}^{3} \text { and } T_{\max } \text { is the } \\
\text { maximum kinetic energy of electrons in } \mathrm{MeV} \text {, taken as the incident photon } \\
\text { energy, which must be }>0.036 \mathrm{MeV} .{ }^{126}\end{array}$ \\
\hline $\begin{array}{l}\text { weighted } \\
\text { sum }\end{array}$ & $\begin{array}{l}d \text { given by } d=\left(f_{\text {photo }} \cdot d_{\text {photo }}\right)+\left(f_{\text {incoh }} \cdot d_{\text {incoh }}\right) \text {, where } f_{\text {photo }}= \\
{\left[1+\left(\frac{\sigma_{\text {incoh }}}{\tau} \cdot \frac{T}{h \nu}\right)\right]^{-1} \text { and } f_{\text {incoh }}=1-f_{\text {photo }} ; \sigma_{\text {incoh }} \text { is the incoherent cross }} \\
\text { section, } \tau \text { is the photoelectric cross section, and } T \text { is the mean Compton } \\
\text { electron energy. Both } d_{\text {photo }} \text { and } d_{\text {incoh }} \text { are given by equation }(4.7) \text { with } \beta \\
\text { given by } e^{-\beta R_{C S D A}}=0.01 ; R_{C S D A} \text { for } d_{\text {photo }}\left(d_{\text {incoh }}\right) \text { corresponds to elec- } \\
\text { trons with energy equal to the incident photon energy } h \nu \text { (the maximum } \\
\text { Compton electron energy). }{ }^{63}\end{array}$ \\
\hline MC estimate & $\begin{array}{l}d \text { is given by } 1-d=\left(D_{c a v}^{\mathrm{NEFW}} / D_{\text {cav }}\right) \text {, where } D_{\text {cav }}^{\mathrm{NEFW}} \text { is the cavity dose due } \\
\text { to electrons originating from within the cavity }(i . e . \text {., NEFW }=\text { No Electrons } \\
\text { From Wall). } D_{\text {cav }}^{\mathrm{NEFW}} \text { is obtained from a version of the EGSnrc user-code } \\
\text { egs_chamber that was modified to discard any electrons generated outside } \\
\text { of the cavity. } D_{\text {cav }} \text { is the cavity dose without any restrictions on electron } \\
\text { origin. }{ }^{127}\end{array}$ \\
\hline
\end{tabular}

The 'Burlin-Chan' and 'Burlin-Janssens' approaches (table 4.1) calculate $\beta$ according to equations of the form $e^{-\beta R}=x$, where $x$ represents the fraction of electrons that reach a depth $R$. The path of an electron is tortuous so that the depth of penetration is less than the total path length, which is generally assumed to be $R_{C S D A}$. Burlin and Chan $^{124}$ suggested $R=F \times R_{C S D A}$, where $F$ is a foreshortening factor; $F=R_{\max } / R_{C S D A} \leq 1$, where $R_{\max }$ is an empirical range. Janssens et al. ${ }^{125}$ suggested using the extrapolated range for $R$. In this study, $F$ is assumed to be unity so that $R=R_{C S D A}$. The 'Burlin-Chan' method uses $x=0.01$, as suggested by Burlin and 
Chan. ${ }^{124}$ Janssens et al. ${ }^{125}$ determined that $x=0.04$ is most appropriate, based on their more rigorous model, which accounts for backscatter at the interface between unlike media; hence $x=0.04$ is used for the 'Burlin-Janssens' method. Values of $R_{C S D A}$, needed for several of the ICT methods, are obtained as described in section 4.2.3.

The 'Loevinger' method was intended to be used for air-filled cavities, and is applicable to energies $>36 \mathrm{keV} .{ }^{126}$ However, the appropriateness of this method has been questioned for the kilovoltage energy range considered herein. ${ }^{124}$ Despite this, recent work has considered this approach for brachytherapy-related applications, e.g., classification of different-sized water cavities, ${ }^{63}$ calculation of doses to thermoluminescent detectors (TLDs) ${ }^{128}$ and electron paramagnetic resonance (EPR) detectors, ${ }^{129}$ and hence it is considered here.

The 'weighted sum' method is based on the work of Carlsson Tedgren and Alm Carlsson, ${ }^{63}$ who considered monoenergetic photon sources between 20 and $300 \mathrm{keV}$, as well as ${ }^{125} \mathrm{I},{ }^{169} \mathrm{Yb}$ and ${ }^{192} \mathrm{Ir}$ brachytherapy sources. They used this approach for source energies between 20 and $100 \mathrm{keV}$; however, we use it over the entire energy range considered herein. The 'weighted sum' method involves computing $d$ as the sum of photoelectron and Compton electron contributions, weighted by $f_{\text {photo }}$ and $f_{\text {incoh }}$, respectively (see table 4.1 ). The coefficients $f_{\text {photo }}$ and $f_{\text {incoh }}$ are calculated using photon cross sections for water; they represent the fraction of energy transferred to electrons that is given to photoelectrons and Compton electrons, respectively.

The 'MC estimate' approach uses MC simulations to obtain a direct estimate of $(1-d)$ according to its fundamental meaning: $(1-d)$ is calculated as the fraction of cavity dose due to electrons originating from within the cavity. The simulation geometry used to calculate $D_{\text {cav }}^{\mathrm{NEFW}}$ and $D_{\text {cav }}$ (the ratio of these quantities gives $(1-d)$ - see table 4.1) consists of a spherical water cavity embedded in a $(2 \mathrm{~cm})^{3}$ cubic water phantom. 


\subsection{Results}

\subsubsection{Evaluation of cavity theory approaches (part I)}

Values of the ICT parameter $d$ (equation (4.6)) evaluated according to the various methods summarized in table 4.1 are presented in figure 4.4 as a function of incident photon energy. For lower energies, $d$ tends toward lower values, the LCT term (the second term) in equation (4.6) dominates, and $R_{C S D A}$ is small compared to the cavity sizes investigated. For higher energies, $d$ tends toward unity for all of the approaches considered, the SCT term dominates, and $R_{C S D A}$ is large compared to cavity dimensions. For a given energy, $d$ values are larger (SCT term more dominant) for smaller $r_{\text {cav }}$. At intermediate energies $($ e.g., between $\sim 20$ and $\sim 200 \mathrm{keV}), d$ values vary considerably between 0 and 1 with photon energy and with ICT approach. For example, $d$ varies between 0.20 and 0.75 at $50 \mathrm{keV}$ for $r_{c a v}=6 \mu \mathrm{m}$. At these intermediate energies, $R_{C S D A}$ values are comparable to or larger than the mean chord lengths of the cavity sizes considered (however, electron displacement may be much shorter than $R_{C S D A}$ due to scattering).

All but two of the ICT approaches produce $d$ values that increase monotonically with energy. 'Weighted sum' and 'MC estimate' both have a local minimum, a local maximum and an inflection point around $50 \mathrm{keV}$. Compton interaction probabilities increase with photon energy so that the electron fluence spectrum has an increasing proportion of relatively low-energy electrons (figure 4.2). In the context of the 'MC estimate' method, lower electron energies have shorter ranges so that a larger fraction of the cavity dose is due to electrons originating from within the cavity; this will increase $D_{\text {cav }}^{\mathrm{NEFW}} / D_{\text {cav }}$, resulting in smaller $d$ values with increasing energy (see equation in table 4.1). For the 'weighted sum' approach, $f_{\text {incoh }}$ increases with energy, $d_{\text {incoh }}$ is generally less than $d_{\text {photo }}$ (because $d_{\text {incoh }}$ corresponds to a lower electron energy), and thus the value of $d$ computed will decrease since a higher weight is given to the smaller $d$ value. 


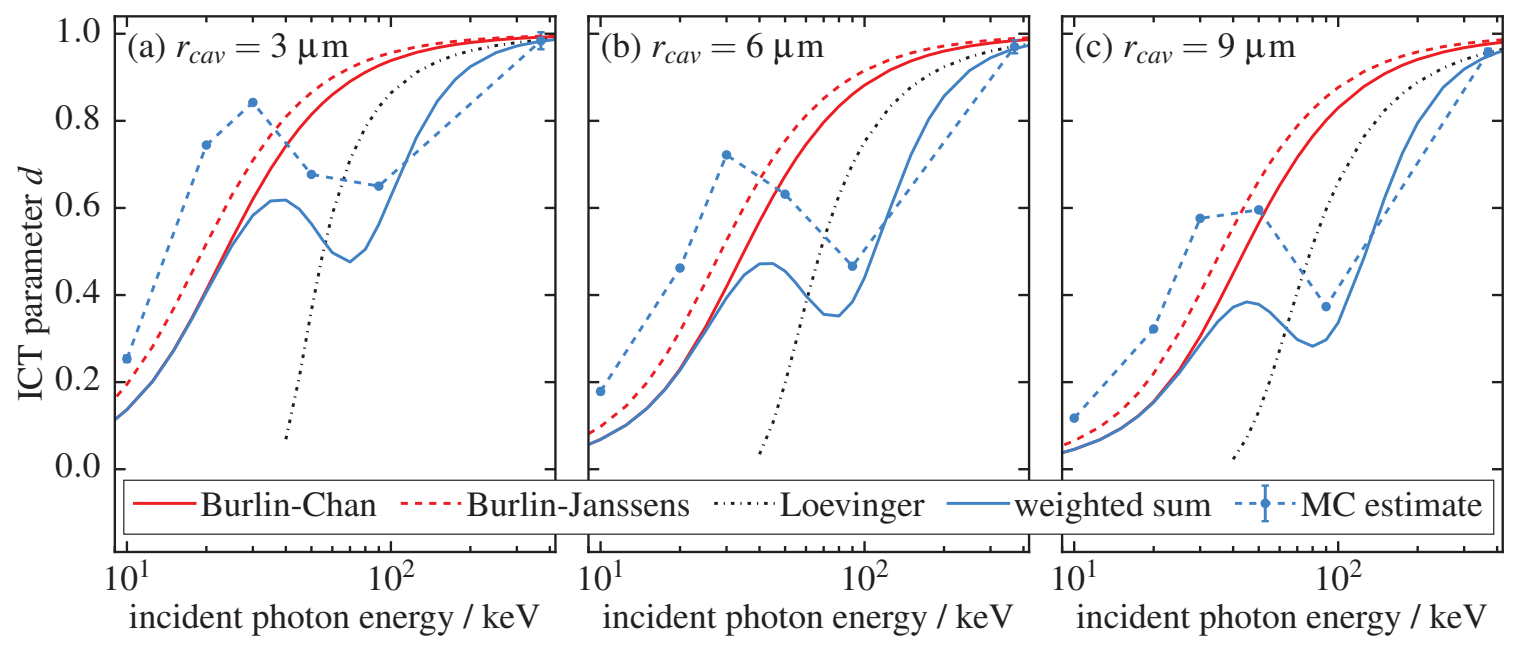

Figure 4.4: Values of the ICT parameter $d$ determined by various approaches (table 4.1) versus incident photon energy for water cavities with radii of (a) 3, (b) 6 and (c) $9 \mu \mathrm{m}$. Values of $d$ are calculated at discrete energies (6 for 'MC estimate', 17 for 'Loevinger', and 25 for other curves), however data points are connected with lines to guide the eye and individual data points are not indicated (except for 'MC estimate'). Error bars on 'MC estimate' values (representing statistical uncertainties) are often too small to see.

Small, large and intermediate cavity theory estimates of $\left(D_{w, m} / D_{m, m}\right)_{C T}$, and MC simulation results for $\left(D_{w, m} / D_{m, m}\right)_{M C}$ for $r_{c a v}=6 \mu \mathrm{m}$ are presented in figure 4.5 for all five bulk tissues. Similar trends in $D_{w, m} / D_{m, m}$ as a function of energy are observed for $r_{c a v}=3$ or $9 \mu \mathrm{m}$ (results not shown). In general, values of $D_{w, m} / D_{m, m}$ are closer to unity for smaller $r_{c a v}$. Trends in $\left(D_{w, m} / D_{m, m}\right)_{M C}$ can be understood by considering how the radiological parameters of the media considered compare to those of water. For lower energies, photoelectric interactions dominate, and photoelectric interaction cross sections of adenoidcystic carcinoma, melanoma and muscle are higher than those of water, resulting in $D_{m, m}$ values larger than $D_{w, m}$. The opposite is true for mammary gland and squamous cell lung carcinoma. Similarly, whether SCT and LCT represent maximum or minimum values may be understood by considering the relative magnitudes of the radiological parameters. For example, mass energy absorption coefficients for mammary gland are lower than those of water, while the opposite is true for the stopping powers (figure 4.3), hence the LCT and SCT values in figure 4.5(b) represent 
the maximum and minimum, respectively. The ICT predictions lie between the LCT and SCT values because $d$ is constrained to lie between 0 and 1 (see equation (4.6)). At higher energies, incoherent interactions dominate, and there are relatively small cross section variations amongst considered media, hence $D_{w, m} / D_{m, m}$ values approach unity. Figure 4.5 demonstrates that differences between MC results and cavity theory predictions vary with phantom material $m$. For example, for mammary gland and squamous cell lung carcinoma, most cavity theories overestimate $\left(D_{w, m} / D_{m, m}\right)_{M C}$, except for SCT. In contrast, for adenoidcystic carcinoma and melanoma, most cavity theories underestimate $\left(D_{w, m} / D_{m, m}\right)_{M C}$, except for SCT.
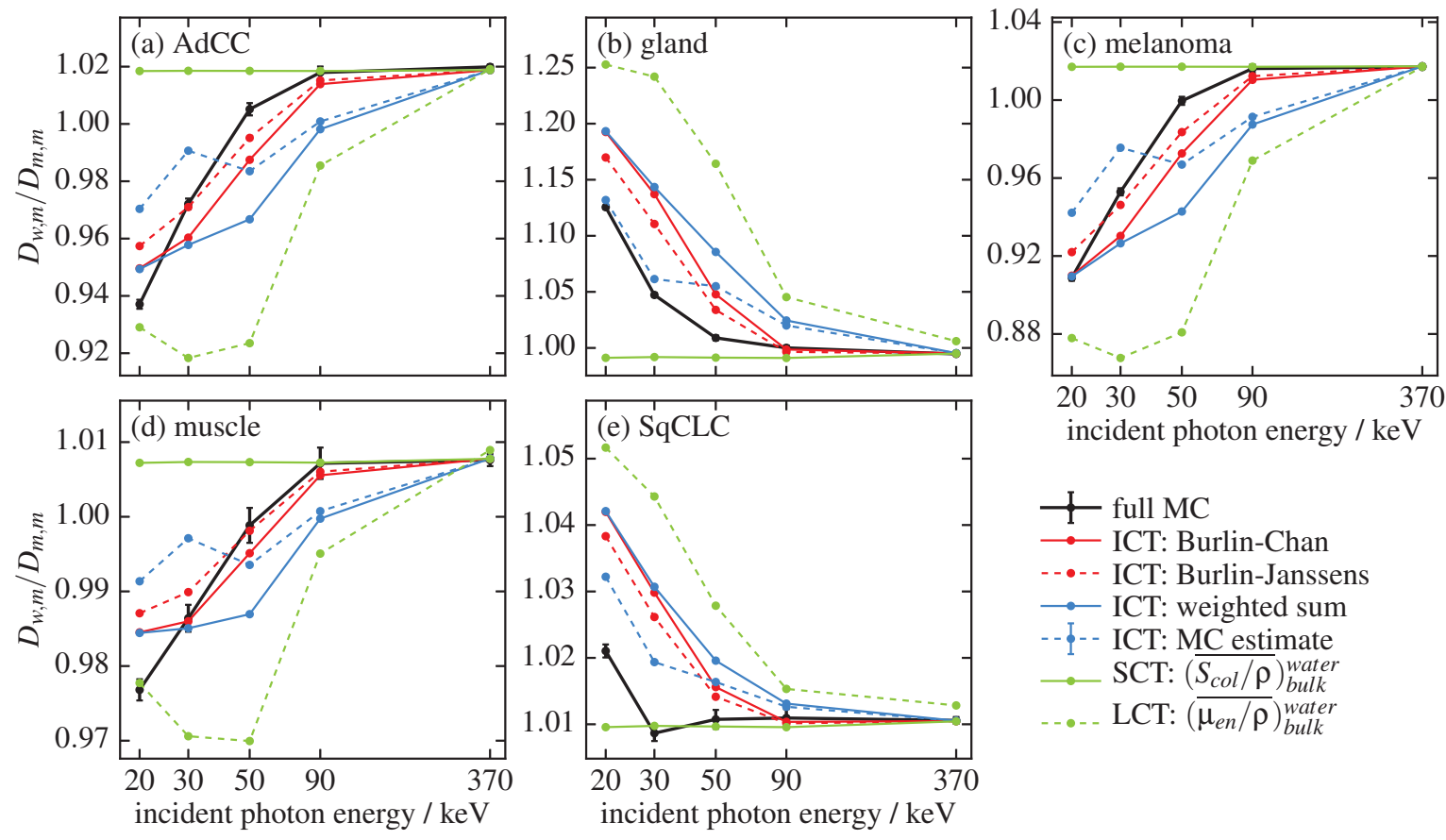

Figure 4.5: $D_{w, m} / D_{m, m}$ is plotted as a function of energy for $r_{c a v}=6 \mu \mathrm{m}$, for five bulk tissues: (a) adenoidcystic carcinoma ('AdCC'), (b) mammary gland ('gland'), (c) melanoma, (d) muscle and (e) squamous cell lung carcinoma ('SqCLC'). $D_{w, m} / D_{m, m}$ is obtained from MC simulations ('full MC'), using various intermediate cavity theory (ICT) methods (table 4.1), or according to small or large cavity theory (SCT or LCT). The ICT dose ratios are calculated according to equation (4.6), where $d$ values for the various ICT methods are presented in figure 4.4. The 'Loevinger' method has been omitted since it is limited to energies $>36 \mathrm{keV}$, and does not provide improved agreement with MC results compared to the other cavity theory approaches. 
The distributions of differences between cavity theory predictions and MC results are presented in figure 4.6 with RMSE values indicated. At $370 \mathrm{keV}$, the cavity theory and MC results are in relatively good agreement in all cases (see figure 4.5), and these data are therefore omitted from histograms but not from RMSE calculations. The largest RMSE values are for SCT and LCT, which is not surprising given that $R_{C S D A}$ values for 20 to $50 \mathrm{keV}$ electrons in the media considered herein vary between 8 and $43 \mu \mathrm{m}$, and are comparable to cellular dimensions. Also, for photon energies above $\sim 50 \mathrm{keV}$, Compton interactions are dominant compared to photoelectric events so that primary electron energies are less than the incident photon energy. Amongst ICT methods, the largest RMSE is for the 'weighted sum' method. The 'Burlin-Janssens' approach achieves the smallest RMSE value and hence closest overall agreement with MC $D_{w, m} / D_{m, m}$ results, with differences of at most $6.0 \%$ across the full range of cavity radii, energies and bulk tissues considered herein (agreement is within $3 \%$ in $92 \%$ of cases). The 'MC-estimate' approach yields a slightly larger RMSE value, but requires a separate MC simulation for each cavity size and source energy; this approach is therefore more involved, especially considering potential applications involving a detailed, voxelized virtual patient model. One might wonder if agreement between MC-computed

$D_{w, m} / D_{m, m}$ and corresponding 'MC estimate' predictions would be improved by using bulk tissue phantoms to estimate $d$ instead of an all-water simulation geometry. Implementing this change causes 'MC estimate' predictions of $D_{w, m} / D_{m, m}$ to be altered by up to $1.4 \%$; however, the RMSE increases slightly rather than being reduced.

\subsubsection{Cavity theory applied to nuclear doses (part II)}

Values of the ICT parameter $d$ computed with the 'Burlin-Janssens' approach are presented in table 4.2 for the nuclear radii considered in multicellular MC simulations. For the lower energy sources, $R_{C S D A}$ values (second column of table 4.2) are comparable to nuclear dimensions, and $d$ is strongly influenced by nucleus size: for cavity radii 

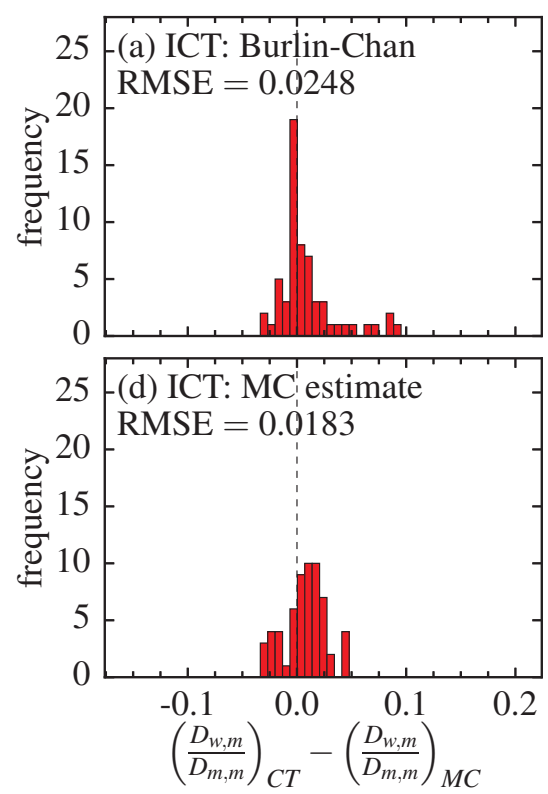
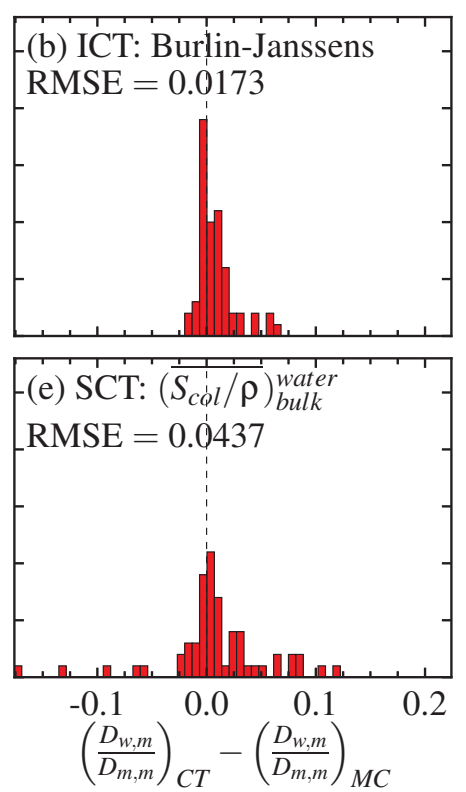
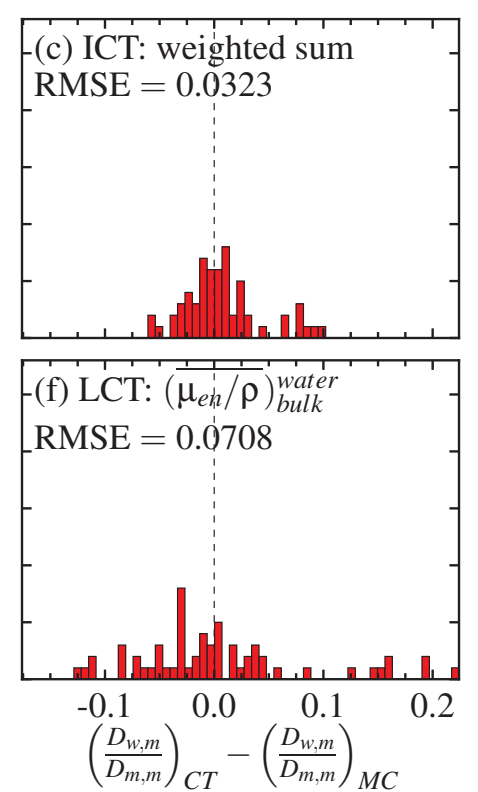

Figure 4.6: Histograms of differences between $D_{w, m} / D_{m, m}$ according to various cavity theory methods (subscript ' $\mathrm{CT}$ ') and the corresponding $\mathrm{MC}$ results (subscript ' $\mathrm{MC}$ ') for four energies (20,30, 50 and $90 \mathrm{keV})$, five bulk tissues and three cavity sizes. Histograms are presented for the (a) 'Burlin-Chan', (b) 'Burlin-Janssens', (c) 'weighted sum', (d) 'MC estimate', (e) small, and (f) large cavity theory approaches. The root mean square error (RMSE) across all energies (including $370 \mathrm{keV}$ ), tissues and cavity sizes is indicated (equation (4.1)). The vertical dashed line indicates the location of zero difference. 
between 2 and $9 \mu \mathrm{m}, d$ varies between 0.616 and 0.208 at $20 \mathrm{keV}$, and between 0.781 and 0.387 at $30 \mathrm{keV}$. As source energy increases, $R_{C S D A}$ increases toward values that are large compared to cellular dimensions, and $d$ is less strongly influenced by nucleus size; $d$ is always within $2 \%$ of unity for the $370 \mathrm{keV}$ photon source.

Table 4.2: Values of $d$ according to the 'Burlin-Janssens' method for five energies and eight cavity radii, $r_{c a v}$, corresponding to the eight nuclear radii considered herein. These values are derived using $R_{C S D A}$ for water (taking into account the assumed nuclear media density of $1.06 \mathrm{~g} / \mathrm{cm}^{3}$ ), motivated by the relatively small differences in $R_{C S D A}\left(\right.$ in $\left.\mathrm{g} / \mathrm{cm}^{2}\right)$ for water and the nuclear media considered herein $(<1.3 \%)$ which result in small differences in $d$ $(<1.2 \%)$. $R_{C S D A}$ values for electrons in water are indicated in the second column, and are calculated according to the methods presented in ICRU Report 37, ${ }^{75}$ using stopping powers from the NIST ESTAR database. ${ }^{76}$

\begin{tabular}{cccccccccc}
\hline \multirow{2}{*}{ Energy $/ \mathrm{keV}$} & \multirow{2}{*}{$R_{C S D A} /\left(\mathrm{g} \cdot \mathrm{cm}^{-2}\right)$} & \multicolumn{8}{c}{$r_{\text {cav }} / \mu \mathrm{m}$} \\
\cline { 3 - 10 } & & 2 & 3 & 4 & 5 & 6 & 7 & 8 & 9 \\
\hline 20 & $8.57 \times 10^{-4}$ & 0.616 & 0.500 & 0.415 & 0.350 & 0.301 & 0.263 & 0.232 & 0.208 \\
30 & $1.76 \times 10^{-3}$ & 0.781 & 0.695 & 0.623 & 0.561 & 0.508 & 0.462 & 0.422 & 0.387 \\
50 & $4.32 \times 10^{-3}$ & 0.902 & 0.858 & 0.816 & 0.778 & 0.741 & 0.708 & 0.676 & 0.646 \\
90 & $1.20 \times 10^{-2}$ & 0.963 & 0.945 & 0.928 & 0.911 & 0.894 & 0.878 & 0.862 & 0.847 \\
370 & $1.15 \times 10^{-1}$ & 0.996 & 0.994 & 0.992 & 0.990 & 0.988 & 0.986 & 0.984 & 0.982 \\
\hline
\end{tabular}

The 'Burlin-Janssens' ICT predictions $\left(\left(D_{n u c, m} / D_{m, m}\right)_{I C T}\right)$ and corresponding MC multicellular simulation results $\left(\left(D_{n u c} / D_{m, m}\right)_{M C}\right)$ are presented in figure 4.7 for the case where $r_{\text {cell }}=7.35 \mu \mathrm{m}$ and $r_{n u c}=4,5$, or $6 \mu \mathrm{m}$; similar trends are observed for other cell and nucleus sizes. Agreement between MC and ICT predictions varies with nucleus/cytoplasm elemental composition, e.g., in figure 4.7(e) ICT predictions and MC results for mammary gland coincide for composition 'a', whereas predictions differ by $5.5 \%$ for composition 'c'. ICT and MC agreement also depends on nuclear radius: for example, for mammary gland at $20 \mathrm{keV}$, the discrepancy between ICT predictions and MC results across all compositions is larger for $r_{n u c}=4 \mu \mathrm{m}$ (figure $4.7(\mathrm{a})$ ) than for $r_{n u c}=6 \mu \mathrm{m}$ (figure $\left.4.7(\mathrm{~g})\right)$. As energy increases, both $\left(D_{n u c, m} / D_{m, m}\right)_{I C T}$ and $\left(D_{n u c} / D_{m, m}\right)_{M C}$ converge toward unity.

In figure 4.8, ICT predictions according to the 'Burlin-Janssens' method are com- 

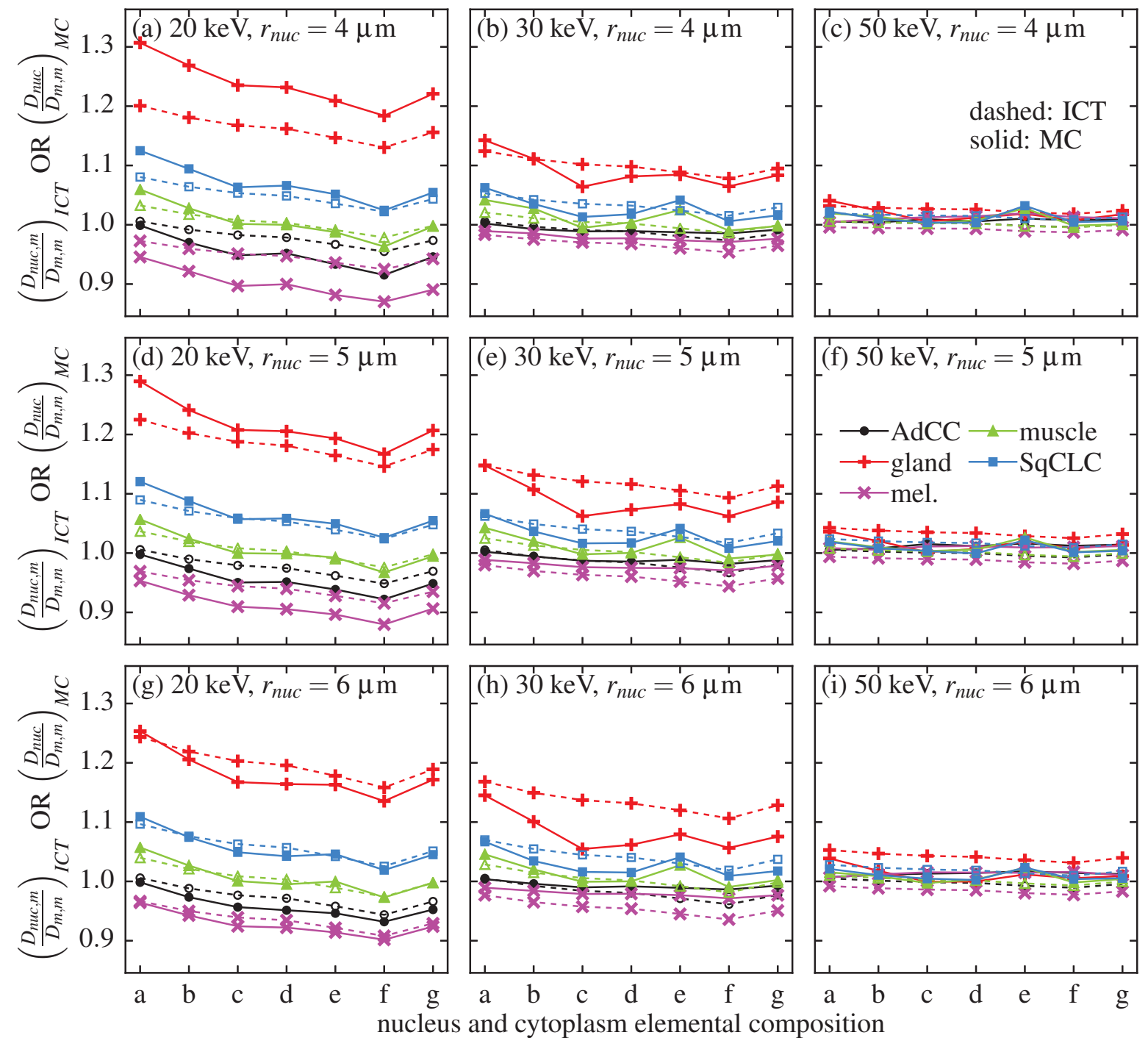

Figure 4.7: ICT predictions according to the 'Burlin-Janssens' method, $\left(D_{n u c, m} / D_{m, m}\right)_{I C T}$, and MC results, $\left(D_{n u c} / D_{m, m}\right)_{M C}$, are presented for $r_{\text {cell }}=7.35 \mu \mathrm{m}$ and three incident photon energies $(20,30$, and $50 \mathrm{keV})$ for nuclear radii: (a,b,c) $4 \mu \mathrm{m}$, (d,e,f) $5 \mu \mathrm{m}$, and $(\mathrm{g}, \mathrm{h}, \mathrm{i}) 6 \mu \mathrm{m}$. Results are presented for five bulk tissues: adenoidcystic carcinoma ('AdCC'), mammary gland ('gland'), melanoma ('mel.'), muscle and squamous cell lung carcinoma ('SqCLC'). Data points are connected with lines to guide the eye. Error bars are often too small to see. Results are presented for 20, 30 and $50 \mathrm{keV}$ incident photons. 
pared with MC simulation results for dose-to-nucleus $/ D_{m, m}$ across the full range of cell/nucleus sizes, cellular elemental compositions and bulk tissues considered herein — four source energies are presented (total of 1260 scenarios). The largest discrepancies are observed for mammary gland, which is an outlier in terms of its radiological parameters (figure 4.3). In figure 4.8(a) (MC simulations involving a cluster of 13 cells), the largest discrepancy between ICT and MC predictions is $8.8 \%$ for gland at $20 \mathrm{keV}$ for $\left(r_{\text {cell }}, r_{n u c}\right)=(5,2) \mu \mathrm{m}$. Across the entire parameter space of 1575 scenarios, 'BurlinJanssens' ICT predictions are in agreement with multicellular MC predictions within $3 \%$ in $91 \%$ of cases. In figure 4.8 (b) (MC simulations involving a single nucleus), the maximum discrepancy is $6.5 \%$ for gland at $30 \mathrm{keV}$ for $r_{n u c}=4 \mu \mathrm{m}$; agreement is within $3 \%$ in $93 \%$ of cases.
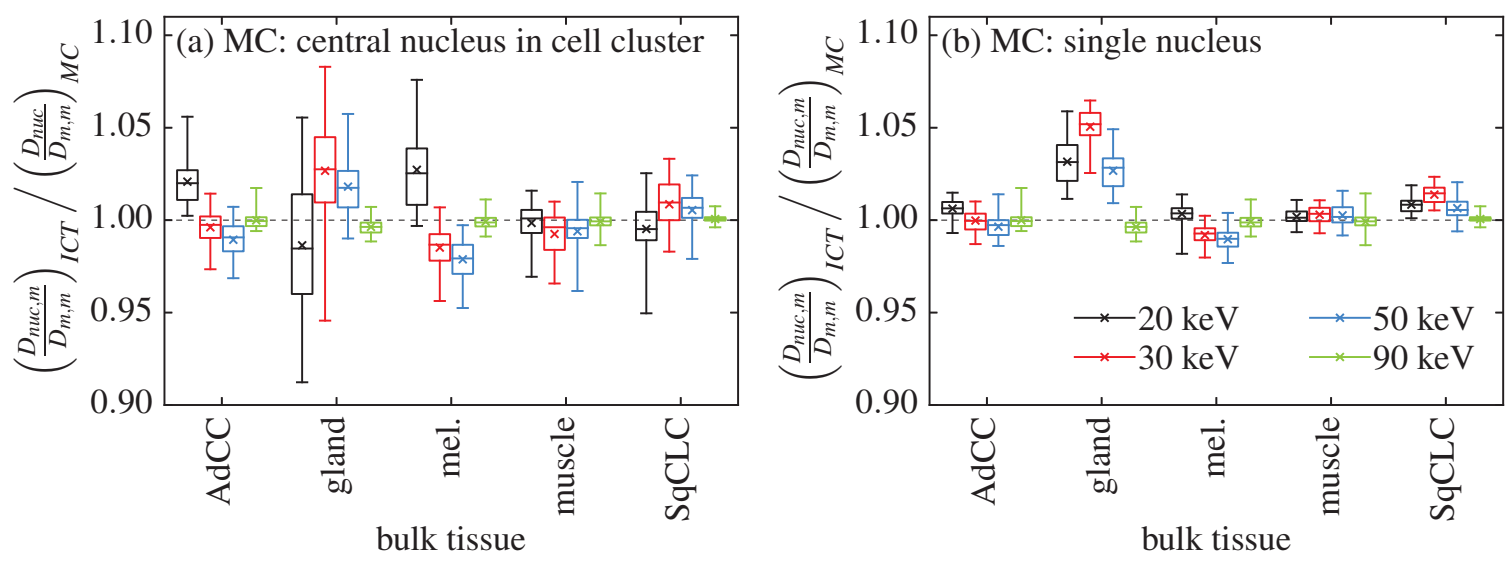

Figure 4.8: Ratios of ICT predictions according to the 'Burlin-Janssens' method (subscript 'ICT') and MC results (subscript 'MC') for dose-to-nucleus $/ D_{m, m}$, for five bulk tissues: adenoidcystic carcinoma ('AdCC'), mammary gland ('gland'), melanoma ('mel.'), muscle and squamous cell lung carcinoma ('SqCLC'). MC results correspond to (a) the central nucleus in a cluster of 13 cells $\left(D_{n u c}\right)$, and (b) a single nucleus $\left(D_{n u c, m}\right)$. Whiskers extend between the minimum and maximum values of the set of results for nine cell/nucleus size combinations and seven cytoplasm/nucleus elemental compositions; box extends between the upper and lower quartiles; its horizontal line indicates the median, and the ' $x$ ' symbol indicates the mean value. Results are presented for 20,30, 50 and $90 \mathrm{keV}$ incident photons. The horizontal dashed lines indicate where the vertical axis is unity. 


\subsubsection{Using Bragg-Gray small cavity theory to estimate dose to small water cavities (part III)}

We finally turn to the application of SCT to estimate dose to small water cavities, motivated by the suggestion that conversion of MBDCA-computed $D_{m, m}$ to $D_{w, m}$ via stopping power ratios (SCT) seems to be the "preferred" conversion method 63 on the basis of the radiobiological importance and universality of DNA-bound water. The two distinct estimates of dose to a small volume of water in nucleus, $D_{w, m}^{S C T}$ and $D_{w, n u c}^{S C T}$ (see figure $4.1(\mathrm{~d}, \mathrm{e}))$, are compared in figure 4.9. The two quantities generally differ for all tissue types, cytoplasm/nucleus elemental compositions, and cell/nucleus sizes. These differences are most pronounced at low energies, e.g., $D_{w, n u c}^{S C T}$ differs from $D_{w, m}^{S C T}$ by up to $35 \%$ for gland at $20 \mathrm{keV}$ when $\left(r_{\text {cell }}, r_{n u c}\right)=(10,7) \mu \mathrm{m}$. Agreement improves with increasing energy. Across the entire parameter space, agreement between $D_{w, n u c}^{S C T}$ and $D_{w, m}^{S C T}$ is within $3 \%$ in $73 \%$ of cases. For muscle, $D_{w, n u c}^{S C T}$ and $D_{w, m}^{S C T}$ agree within $7 \%$ for all cases.

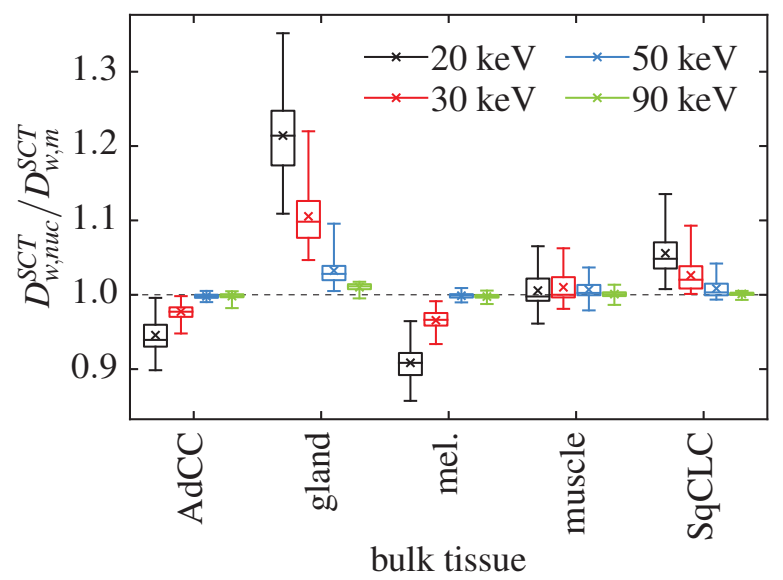

Figure 4.9: Ratios of dose estimates for small volumes of water according to BraggGray small cavity theory (see equation (4.2)), based on MC results for either $D_{n u c}$ or $D_{m, m}$, for five bulk tissues: adenoidcystic carcinoma ('AdCC'), mammary gland ('gland'), melanoma ('mel.'), muscle and squamous cell lung carcinoma ('SqCLC'). See figure 4.8 for an explanation of the box and whisker plots. Results are presented for 20, 30, 50 and $90 \mathrm{keV}$ photons. The horizontal dashed line indicates where the vertical axis is unity. 


\subsection{Discussion}

\subsubsection{Evaluation of cavity theory approaches (part I)}

Figure 4.4 demonstrates the wide range of $d$ values that can be obtained depending on choice of ICT approach, underlining the importance of exploring the full range of possible cavity theory options (e.g., including comparison with MC results or experimental data, if available) before choosing an approach for a particular scenario. Carlsson Tedgren and Alm Carlsson ${ }^{63}$ presented $d$ values for kilovoltage photon sources and cavity sizes from $1 \mathrm{~nm}$ to $1 \mathrm{~mm}$, considering an extrapolated version of the 'Loevinger' approach and two 'weighted sum' methods involving different equations for determining $\beta$. For all three approaches, their $d$ values are within the range of values presented in figure 4.4 (considering $\mu \mathrm{m}$-sized cavities). They also provided $D_{w, m} / D_{m, m}$ conversion factors for $1 \mathrm{~nm}$ to $1 \mathrm{~mm}$ cavities using a single 'weighted sum' ICT approach selected based on comparison with two data points taken from the MC simulation results (involving a $7 \mu \mathrm{m}$ radius cavity) of Enger et al. ${ }^{55}$ The results of this chapter support the 'Burlin-Janssens' approach over the 'weighted sum' method for kilovoltage photon energies and cellular $(\mu \mathrm{m})$ length scales. However, further tests involving a larger range of cavity sizes and media are needed to establish a more universally-applicable approach for calculating conversion factors that relate macro- and microscopic dose descriptors. Furthermore, assigning different cavity theory approaches to different regions of the parameter space (e.g., different energies, bulk tissues, cavity sizes, and cavity media) could potentially lead to improved agreement between cavity theory predictions and $\mathrm{MC}$ results. For example, if each bulk tissue is considered separately in the comparison of MC and cavity theory results, then the RMSE is minimized with 'MC estimate' for mammary gland, 'Burlin-Chan' for muscle, SCT for squamous cell lung carcinoma, and 'Burlin-Janssens' for adenoidcystic carcinoma and melanoma. However, such approaches introduce additional complexity. 
For the 'weighted sum' approach, $f_{\text {photo }}$ and $f_{\text {incoh }}$ are computed using photon interaction cross sections corresponding to the incident photon energy, ${ }^{63}$ ignoring the full spectrum of photon energies present at depth in the phantom. To investigate the effect of considering the spectrum of energies, we additionally carried out MC simulations to calculate $f_{\text {photo }}$ and $f_{\text {incoh }}$. These MC-computed $f$-values result in $d$-values that differ by at most $3.4 \%$ from the $d$-values obtained using the photon cross sections, with the largest discrepancies observed at 50 and $90 \mathrm{keV}$, where Compton interactions begin to dominate over photoelectric interactions and an increasing number of scattered photons are produced. The low probability of photoelectric interactions above $100 \mathrm{keV}$, and Compton interactions below $10 \mathrm{keV}$ result in considerable uncertainties in MC-computed $f_{\text {photo }}$ and $f_{\text {incoh }}$ unless a very high number of histories is used.

In the context of higher energy photon beams (1 and $10 \mathrm{MeV})$, Kumar et al. ${ }^{130}$ used the ratio $D_{S-A} / D_{M C}$ to calculate the ICT parameter $d$, where $D_{S-A}$ is the SpencerAttix absorbed dose (i.e., the numerator of equation (4.5)), with $\Delta$ taken as the energy of an electron that can just cross the cavity, and $D_{M C}$ is the corresponding MC result for the cavity dose. Their method is analogous to our 'MC estimate' method, with $D_{S-A} / D_{M C}$ representing the fraction of energy deposition due to electrons with energy $>\Delta$, corresponding to ranges that are larger than cavity dimensions (i.e., electrons originating from the wall; $D_{S-A} / D_{M C}$ is equivalent to $1-\left(D_{c a v}^{\mathrm{NEFW}} / D_{\text {cav }}\right)-$ see table 4.1). Their MC-based $d$ estimate was found to agree reasonably well with $d$ determined according to the 'Burlin-Janssens' approach in which they used $R_{C S D A}$ corresponding to the maximum Compton electron energy resulting from the average bremsstrahlung photon energy.

\subsubsection{Cavity theory applied to nuclear doses (part II)}

The application of the 'Burlin-Janssens' ICT method for predictions of nuclear dose yields results in relatively good agreement with those of multicellular MC simulations, 
with agreement within $3 \%$ in $91 \%$ of cases (maximum discrepancy of $8.8 \%$ ) over the parameter space of 1575 scenarios. Furthermore, this level of agreement is comparable to that achieved when considering a single water cavity in an otherwise homogeneous bulk tissue phantom (part I: agreement within $3 \%$ in $92 \%$ of cases, maximum discrepancy of $6.0 \%$ ). Our results suggest that ICT is necessary for conversion between macroscopic and cellular (nuclear) dose descriptors for kilovoltage photon energies; $R_{C S D A}$ values of electrons set into motion by kilovoltage photons are comparable to cellular dimensions so that neither SCT nor LCT is generally appropriate.

The cavity theories investigated in this chapter assume that the cavity is surrounded by an infinite, homogeneous medium so that, in principle, $\left(D_{n u c, m} / D_{m, m}\right)_{M C}$ values (single nucleus in a homogeneous phantom - figure 4.1(c)) should match the predictions of cavity theory more closely than $\left(D_{n u c} / D_{m, m}\right)_{M C}$ (multicellular model figure 4.1(b)). Indeed, $\left(D_{n u c, m} / D_{m, m}\right)_{C T}$ is in better agreement with $\left(D_{n u c, m} / D_{m, m}\right)_{M C}$ than $\left(D_{n u c} / D_{m, m}\right)_{M C}$ : the RMSE decreases by $13.2 \%$, and the maximum deviation from unity in figure 4.8 decreases from $8.8 \%$ to $6.5 \%$ when only a single nuclear cavity is simulated. In the previous chapter, it was demonstrated that the presence of a cytoplasm, surrounding cells and ECM perturbs the nuclear dose by up to $13.0 \%$ (with the cytoplasm having the largest effect), which supports this improved agreement. Almond and Svensson ${ }^{131}$ presented a SCT expression accounting for both a wall next to the cavity and a build-up cap surrounding the wall. Future work could investigate the use of this two-component model in the context of cellular dosimetry with the cytoplasm as the wall and bulk tissue as the build-up cap, using ICT instead of SCT. However, Buckley et al. ${ }^{132}$ demonstrated that this two-component model fails to predict ion chamber response in a ${ }^{60} \mathrm{Co}$ beam. 


\subsubsection{Using Bragg-Gray small cavity theory to estimate dose to small water cavities (part III)}

In figure 4.9, the conventional approach of taking MC-computed $D_{m, m}$ and applying mass collisional stopping power ratios (water:medium) to obtain a SCT estimate of dose-to-water-in-medium, $D_{w, m}^{S C T}$ (figure 4.1(d)), is contrasted with using MC-computed $D_{n u c}$ and applying mass collisional stopping power ratios (water:nucleus) to obtain a MC-SCT estimate of dose-to-water-in-nucleus, $D_{w, n u c}^{S C T}$ (figure 4.1(e)). In considering these results, we may ask: if a $\sim$ nm-sized, DNA-bound cluster of water molecules in the nucleus is a radiobiologically-significant target, is $D_{w, m}^{S C T}$ an accurate estimate of energy deposition within such a volume?

Previous MC simulations involving multicellular models demonstrate that the microscopic tissue structure affects doses to subcellular targets (see ${ }^{35}$ and chapter 3), suggesting the need for further research to understand the appropriateness of $D_{w, m}^{S C T}$. $D_{w, n u c}^{S C T}$ is based on MC simulations that explicitly account for the microscopic tissue structure in terms of cells and their nuclei. However, accurate determination of energy deposition within $\sim$ nm-sized volumes would require more detailed geometric models of cells and subcellular structure, as well as modelling of radiation transport down to sub-1 keV energies, which presents various challenges ${ }^{133}$ - this is beyond the scope of this chapter. Nonetheless, the results in figure 4.9 demonstrate the sensitivity of $D_{w, n u c}^{S C T}$ to the simulation geometry (structure, composition) considered. The microscopic tissue structure has an increasingly large effect on energy deposition within subcellular compartments with decreasing energy; at higher energies, there is little sensitivity to tissue structure detail (see results presented in chapter 3). Estimating dose to a small volume of water based on SCT conversion from $D_{m, m}$ yields a result that differs from the estimate based on SCT conversion from $D_{n u c}$ by up to $35 \%$ (figure 4.9). These results suggest that the application of SCT to MC-computed $D_{m, m}$ to compute dose 
to a small volume of water in medium may not be well motivated as an estimate of energy deposition within DNA-bound water in the nucleus for all source energies and tissue types. In terms of the debate over the medium for dose specification for MBDCA treatment planning and evaluation, ${ }^{31,32}$ these considerations do not support adoption of dose-to-water involving conversion from bulk tissue dose $\left(D_{m, m}\right)$ using SCT.

\subsection{Conclusions}

This chapter presents an investigation of seven cavity theory methods (five ICT approaches presented in the literature, plus SCT and LCT) in the context of cellular dosimetry for kilovoltage photon energies. Considering three different $\mu$ m-sized water cavities in five different bulk tissues and five source energies, comparisons of cavity theory predictions and $\mathrm{MC}$ results for $D_{w, m} / D_{m, m}$ demonstrate best overall agreement (minimum RMSE) with the 'Burlin-Janssens' ICT approach. When this approach is used to estimate nuclear doses in multicellular MC simulations, differences are $<3 \%$ in $91 \%$ of cases across the parameter space of nine cell/nucleus size combinations, seven cytoplasm/nucleus elemental compositions, five bulk tissues and five source energies. Therefore, cavity theory may be useful for linking the macroscopic dose descriptors used in clinical treatment plans and radiation protection simulations (i.e., $D_{m, m}$ ) with energy deposition in $\mu \mathrm{m}$-sized targets across a range of different cell morphologies and compositions. Regarding the debate over the dose specification medium for MBDCAs, if the nucleus is considered the biologically-relevant target, then the results of this chapter demonstrate that ICT techniques are needed to convert from $D_{m, m}$ to $D_{n u c}$ at kilovoltage photon energies. Considering a small volume of water in the nucleus as the target of interest, our findings generally do not support SCT conversion of $D_{m, m}$ to $D_{w, m}$, underlining the importance of the microscopic structure surrounding the target of interest. The scope of the work in this chapter is limited to monoenergetic photon sources; for spectral sources, cavity theory conversion factors for monoenergetic sources 
can be combined using appropriate weighting factors. Future research may continue to investigate cavity theory approaches for converting MBDCA-computed macroscopic dose descriptors to biologically-relevant quantities, and will need to consider the range of pathways to radiation-induced cell death (and variety of possible associated targets) that have been reported in the literature. 


\section{Chapter 5}

\section{Multiscale Monte Carlo simulations investi- gating energy deposition within cell popula- tions}

Variation in energy deposition across a population of cells due to the stochastic nature of radiation transport and energy deposition is investigated using multiscale Monte Carlo simulation models, which are an extension of the multicellular models used in the previous two chapters. This chapter explores how patterns of energy deposition depend on incident photon energy, dose level, and microscopic tissue structure model details (choice of media, cell arrangement method, and cell/nucleus size). 


\subsection{Introduction}

This chapter investigates energy deposition in subcellular targets using multiscale models of normal and cancerous human soft tissues, which combine varying levels of detail on different length scales. MC simulations are used to calculate specific energy distributions, $f(z, D)$, for nuclear and cytoplasm targets in populations of cells. We investigate how these $f(z, D)$ depend on incident photon energy, dose level, tissue elemental composition, cell/nucleus size, and cell arrangement method. Photon energies ranging from $20 \mathrm{keV}$ up to a ${ }^{60} \mathrm{Co}$ spectrum, and $\mathrm{mGy}$ to Gy dose levels are considered, which are relevant for brachytherapy, megavoltage external beam out-of-field radiation, and diagnostic radiology (where typical doses include $\sim 3$ mGy for a mammogram, $\sim 10$ mGy for abdominal computed tomography (CT), and 40 to 100 mGy for a CT coronary $\left.\operatorname{angiogram}^{11}\right)$.

\subsection{Methods}

\subsubsection{Development of microscopic tissue structure models}

Multiscale tissue models are developed since it is not feasible to model microscopic detail throughout the entire simulation geometry. These involve a microscopically-detailed region of interest $(\mathrm{ROI})$ comprised of $>1500$ cells embedded at the centre of a $(2 \mathrm{~cm})^{3}$ bulk tissue phantom. Each cell is modelled as two concentric spheres with cytoplasm and nucleus compartments. Cells are embedded in an extracellular matrix (ECM). Cell and nucleus sizes are based a literature survey of human cell and nucleus sizes (see table $2.2)$ : three nominal cell radii are considered $\left(r_{\text {cell }}=5,7.5,10 \mu \mathrm{m}\right)$; two nominal nuclear radii are considered for each of these $\left(r_{n u c}=[2,4],[3,6],[4,8] \mu \mathrm{m}\right.$, respectively).

Four methods for cell arrangement and assignment of cell/nucleus size are consid- 


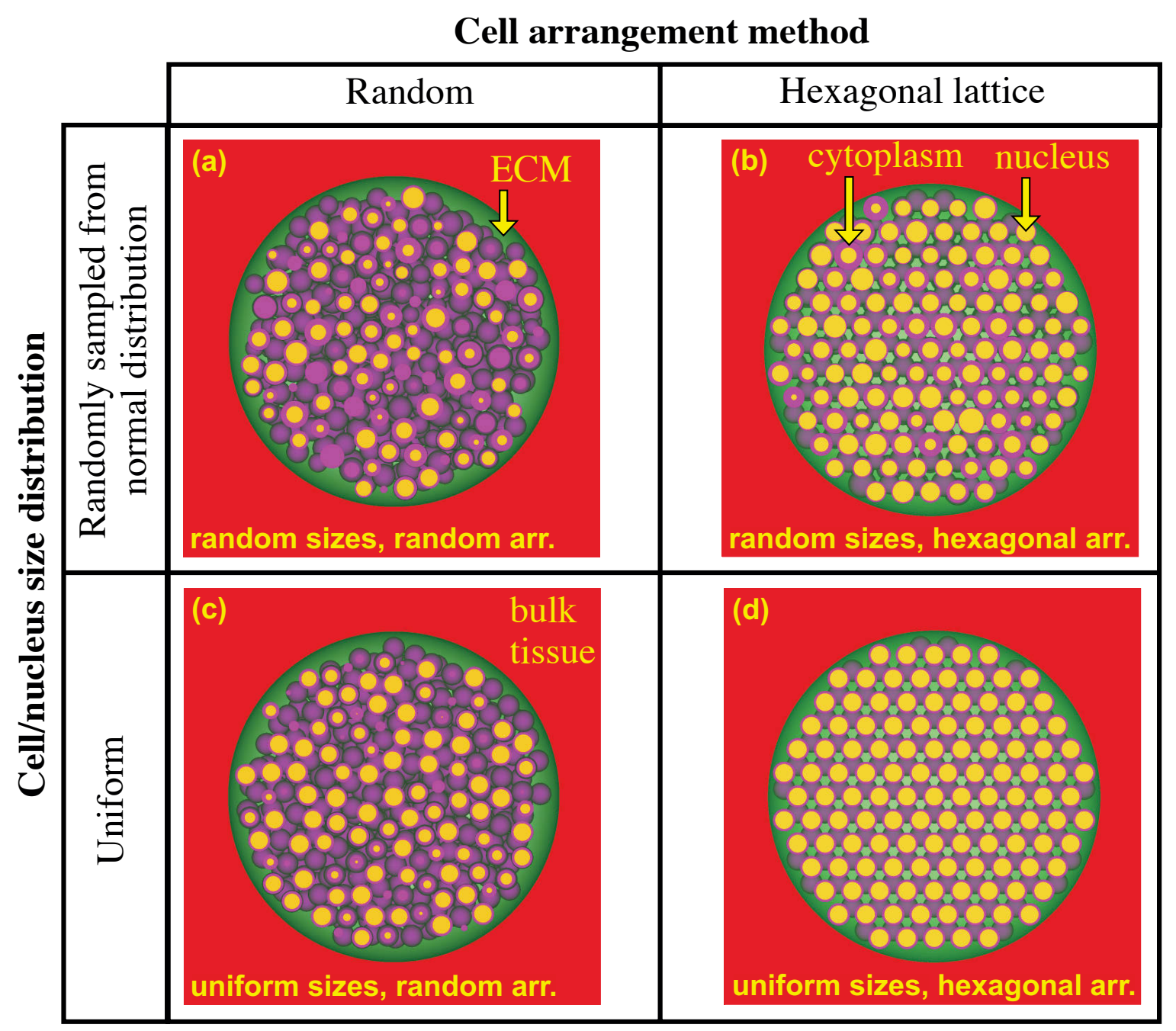

Figure 5.1: cross sections of microscopic tissue structure models containing $>1500$ cells embedded in a sphere of extracellular matrix (ECM) (radius 89 to $161 \mu \mathrm{m}$ ). Two cell arrangement methods ('random arrangement' or 'hexagonal arrangement') and two cell/nucleus size distributions ('random sizes' and 'uniform sizes') are considered. Each of these is positioned at the centre of a $(2 \mathrm{~cm})^{3}$ cubic bulk tissue phantom. 
ered (figure 5.1). Cell and nucleus sizes may be uniform (figure 5.1(c,d)) or random (figure 5.1(a,b)), as determined via sampling from a normal distribution of $1 \mathrm{\mu m}$ standard deviation (where this value is motivated by uncertainties on cell and nuclear radii for six breast cancer cell lines ${ }^{102}$ ). Cells may be arranged in a hexagonal lattice (figure $5.1(\mathrm{~b}, \mathrm{~d})$ ) or have random positions (figure 5.1(a,c)) determined from a pouring simulation carried out using the LAMMPS Molecular Dynamics Simulator, ${ }^{134}$ which was modified to allow for the option of sampling sphere sizes from a normal distribution. The nearest neighbour distance is taken to be $2.06 \mu \mathrm{m}$ for this 'random arrangement' to achieve cell number densities falling within the range of typical values observed for human soft tissues. ${ }^{35}$ For cells in 'hexagonal arrangement', this distance is adjusted to $2.93,3.22$, and $3.16 \mu \mathrm{m}$ (for cell radii of $5,7.5$, and $10 \mu \mathrm{m}$, respectively) to achieve approximately the same cell number density as the corresponding 'random arrangement'. Most results are presented for the 'random sizes, random arrangement' model (figure 5.1(a)) since it is considered the most realistic; however, the other models (figures 5.1(bd)) enable investigation of how cell arrangement method, and the distribution of cell and nucleus sizes influence energy deposition within subcellular compartments.

For the models with 'random' cell/nucleus sizes, actual mean radii may differ from nominal values (figure 5.2; table 5.1). This is because values of the nuclear radius, $r_{n u c}$, are sampled after cell radii, $r_{c e l l}$, have already been determined; the constraints $r_{n u c} \leq r_{\text {cell }}-0.5 \mu \mathrm{m}$ and $r_{n u c} \geq 0.5 \mu \mathrm{m}$ means that values of $r_{n u c}$ sometimes need to be resampled. The cell radius is also required to be within four standard deviations of the mean to avoid the occurrence of extreme outliers. Additionally, some cell radii in the 'random sizes, hexagonal arrangement' case were adjusted to slightly smaller values in order to avoid overlapping cells. For simplicity, nominal radii are used to label results.

Five bulk tissues are considered: two normal tissues (mammary gland ${ }^{84}$ and mus$\mathrm{cle}^{85}$ ) and three cancerous tissues (adenoidcystic carcinoma, melanoma, squamous cell 

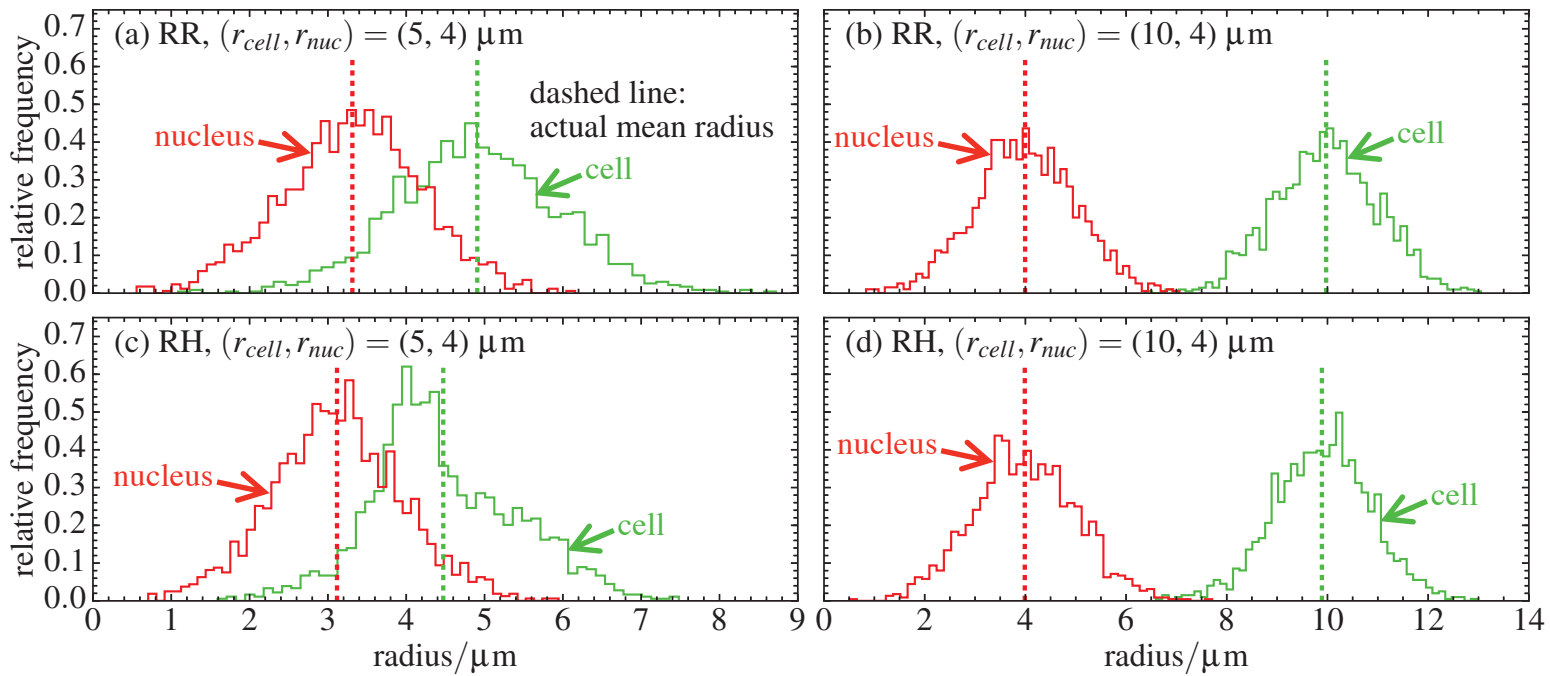

Figure 5.2: Distributions of cell and nucleus radii in cell populations for nominal values $(\mathrm{a}, \mathrm{c})\left(r_{\text {cell }}, r_{n u c}\right)=(5,4)$ and $(\mathrm{b}, \mathrm{d})\left(r_{\text {cell }}, r_{n u c}\right)=(10,4) \mu \mathrm{m}$, for $(\mathrm{a}, \mathrm{b})$ 'random sizes, random arrangement' ('RR') and (c,d) 'random sizes, hexagonal arrangement' ('RH'). Actual mean radii are indicated by the vertical dashed lines.

Table 5.1: Nominal and actual mean cell and nucleus ('nuc.') radii, and actual mean cytoplasm ('cyt.') and nucleus volumes ('vol.') for the 'random sizes, random arrangement' ('RR') and 'random sizes, hexagonal arrangement' ('RH') cases. The actual mean volume is obtained by averaging across the actual volumes of each target in the population.

\begin{tabular}{|c|c|c|c|c|c|c|c|c|c|}
\hline \multirow{2}{*}{\multicolumn{2}{|c|}{ Nominal radii/um }} & \multicolumn{4}{|c|}{ Actual mean radii/um } & \multicolumn{4}{|c|}{ Actual mean vol. $/ \mathrm{um}^{3}$} \\
\hline & & \multicolumn{2}{|c|}{$\mathrm{RR}$} & \multicolumn{2}{|c|}{$\mathrm{RH}$} & \multicolumn{2}{|c|}{$\mathrm{RR}$} & \multicolumn{2}{|c|}{$\mathrm{RH}$} \\
\hline cell & nuc. & cell & nuc. & cell & nuc. & cyt. & nuc. & cyt. & nuc. \\
\hline 5 & 2 & 4.9 & 2.1 & 4.5 & 2.0 & $5.0 \times 10^{2}$ & $5.7 \times 10^{1}$ & $3.7 \times 10^{2}$ & $5.1 \times 10^{1}$ \\
\hline 5 & 4 & 4.9 & 3.3 & 4.5 & 3.1 & $3.8 \times 10^{2}$ & $1.8 \times 10^{2}$ & $2.7 \times 10^{2}$ & $1.5 \times 10^{2}$ \\
\hline 7.5 & 3 & 7.5 & 3.0 & 7.2 & 3.0 & $1.7 \times 10^{3}$ & $1.5 \times 10^{2}$ & $1.5 \times 10^{3}$ & $1.5 \times 10^{2}$ \\
\hline 7.5 & 6 & 7.5 & 5.6 & 7.2 & 5.5 & $1.1 \times 10^{3}$ & $7.9 \times 10^{2}$ & $9.4 \times 10^{2}$ & $7.3 \times 10^{2}$ \\
\hline 10 & 4 & 10.0 & 4.0 & 9.9 & 4.0 & $4.0 \times 10^{3}$ & $3.2 \times 10^{2}$ & $3.8 \times 10^{3}$ & $3.2 \times 10^{2}$ \\
\hline 10 & 8 & 10.0 & 7.7 & 9.9 & 7.7 & $2.3 \times 10^{3}$ & $2.0 \times 10^{3}$ & $2.2 \times 10^{3}$ & $2.0 \times 10^{3}$ \\
\hline
\end{tabular}


lung carcinoma ${ }^{86}$ ) with elemental compositions and mass densities presented in table 2.1 (using the modified versions of mammary gland, muscle and squamous cell lung carcinoma, as described below). The ECM elemental composition and density are chosen such that the average, mass-weighted composition and density of the cells plus surrounding ECM is equal to that of the corresponding bulk tissue (for self-consistency). For the purpose of calculating the ECM composition for each cell/nucleus size combination and bulk tissue, an infinite hexagonal lattice of uniformly-sized cells with a minimum separation distance of $2.06 \mu \mathrm{m}$ is assumed. For some of the bulk tissues considered herein, the self-consistency requirement described above cannot be satisfied. To remedy this issue, the phosphorus and potassium mass fractions for mammary gland, muscle and squamous cell lung carcinoma are increased slightly. Modified mammary gland and muscle have water, lipid, protein and ash (i.e., inorganic) content that are within the range of observations reported by Woodard and White. ${ }^{84}$ Modified squamous cell lung carcinoma has elemental mass fractions and ash content that are within the range of values established by the two samples presented in Maughan et al. ${ }^{86}$ The resulting changes in radiological parameters are shown in figure 2.2. Seven representative cytoplasm/nucleus elemental compositions were considered in previous chapters; in this chapter, only cytoplasm and nucleus compositions 'g' are considered. Composition 'g' corresponds to normal lactating mouse mammary epithelial cells, based on data from Cameron et al. ${ }^{92}$

\subsubsection{Monte Carlo simulations and analysis of results}

Simulations are carried out using a version of the EGSnrc user-code egs_chamber 28,72 that is modified to allow scoring energy deposition in multiple regions. The source is a parallel beam of photons; five monoenergies are considered (20, 30, 50, 90 and $370 \mathrm{keV})$,

in addition to a Cobalt-60 spectrum. ${ }^{135}$ The beam has a circular cross section with a radius of $1 \mathrm{~cm}$, incident on the side of the $(2 \mathrm{~cm})^{3}$ phantom. 
Specific energies, $z$, to cell nucleus and cytoplasm targets are computed for various corresponding doses, $D_{m, m}$, defined as the dose to a sphere of homogeneous bulk tissue occupying the space of the cell population and ECM. The mean specific energy $(\bar{z})$, standard deviation of the specific energy $\left(\sigma_{z}\right)$ and fraction of targets receiving no energy deposition $(f(z=0))$ are determined from the specific energies of all targets (nuclei or cytoplasms) in a cell population. Specific energy distributions $f(z, D)$ are normalized such that $\int_{0}^{\infty} f(z, D) d z=1$. As cellular targets and bulk tissues differ in elemental composition, $\bar{z} \neq D_{m, m}$ in general. MC simulations are split up across hundreds of jobs on different CPUs, each one achieving its own $D_{m, m}$. To obtain results for a particular dose level (i.e., value of $\left.D_{m, m}\right)$, results from different jobs are added up, resulting in limited dose resolution. In section 5.3, the actual $D_{m, m}$ is indicated where possible; otherwise, an approximate value is given (e.g., $D_{m, m} \simeq 10 \mathrm{mGy}$ ).

Unlike cells on the interior of the ROI, cells on the outside are not completely surrounded by neighbouring cells (figure 5.1), which may affect energy deposition within them, and thus these cells are excluded from data analysis. Therefore, although $>1500$ cells are simulated, specific energy is scored in the central $\sim 1000$ cells only.

In total, six incident photon energies, five bulk tissues, six cell/nucleus size combinations, two cell arrangement methods, two cell/nucleus size distributions, and various dose levels are considered, for both nucleus and cytoplasm targets. Given this extensive parameter space, only a subset of MC results are presented in section 5.3, often only explicitly showing results where melanoma is the bulk tissue (arbitrary choice). Additional results are summarized in tables in appendix B.

In addition to presenting results (i.e., for $f(z, D), \bar{z}$ and $\sigma_{z}$ ) obtained directly from MC simulations, we also consider two analytic expressions: (I) normal distributions having the same mean and standard deviation as $f(z, D)$, and (II) an equation relating the relative standard deviation of the specific energy $\sigma_{z} / \bar{z}$ to the dose $D_{m, m}$ and the 
target volume. In both cases, the root mean square error (RMSE) compared to MC simulation results is calculated as follows:

$$
\mathrm{RMSE}=\sqrt{\frac{1}{N}\left[\sum_{i=1}^{N}\left(x_{i, \text { compare }}-x_{i, M C}\right)^{2}\right]}
$$

where $N$ is the number of data points, and $x_{i, c o m p a r e}$ and $x_{i, M C}$ are described below.

To evaluate how closely the MC-calculated $f(z, D)$ agrees with the corresponding normal distribution for (I), $x_{i, \text { compare }}$ and $x_{i, M C}$ in equation 5.1 are the values of the specific energy distribution according to the normal distribution (evaluated at the bin centre) and $\mathrm{MC}$ simulation, respectively. The sum is over the $N$ bins in the histogram representing $f(z, D)$. For the purpose of calculating the RMSE, a bin width of $1 \mathrm{mGy}$ is used, with bins spanning the range within three standard deviations of the mean (the range will start at zero if the mean less three standard deviations is a negative number).

The expression for (II) is based on equations (2.19) and (A.12) from ICRU Report $36,{ }^{136}$ which can be used to derive a functional form of

$$
\sigma_{z} / \bar{z}=\frac{C_{1}}{V^{C_{2}} \sqrt{D_{m, m}}}
$$

to approximately relate microdosimetric spread (i.e., $\left.\sigma_{z} / \bar{z}\right)$ to target volume $V$ in $\mu \mathrm{m}^{3}$, and dose $D_{m, m}$ in Gy, in agreement with Villegas et al. ${ }^{64}$ Using MC simulation results for the 'random sizes, random arrangement' case (figure 5.1(a)) and the actual mean target volume (table 5.1), the curve_fit function from the optimize package of the SciPy library of the Python programming language (http://www.python.org) is used to determine different $C_{1}$ and $C_{2}$ fit parameters for different incident photon energies; nuclear and cytoplasm targets are considered separately. The fitting procedure is applied across the parameter space consisting of all cell/nucleus sizes, bulk tissues, and doses > $10 \mathrm{mGy}$. The low dose threshold of 10 mGy is motivated by the fact that thresholds $\geq 10$ mGy 
yield similar fit parameters, whereas fit parameters obtained with thresholds $<10$ mGy varied considerably. The maximum available dose ranges from 60 mGy up to 1 Gy. For case (II), $x_{i, c o m p a r e}$ and $x_{i, M C}$ in equation 5.1 are the values of $\sigma_{z} / \bar{z}$ (for scenario $i$ ) according to equation 5.2 and MC simulation, respectively; the sum is over the entire parameter space. The RMSE is calculated for each incident photon energy, for nuclear and cytoplasm targets.

\subsection{Results}

Example specific energy distributions for nuclear targets in cell populations are presented in figure 5.3, demonstrating variation with cell/nucleus size and source energy. In general, for larger target sizes and higher energies, the fraction of targets receiving no energy deposition is smaller, the shape of $f(z, D)$ becomes less skewed (appearing closer to that of a normal distribution) and the microdosimetric spread is smaller this is also true for higher doses (results not shown). An exception to these general trends with incident photon energies occurs at $90 \mathrm{keV}: \sigma_{z} / \bar{z}$ and $f(z=0)$ at $90 \mathrm{keV}$ is larger than at $50 \mathrm{keV}$ for all cell/nucleus sizes (discussed further in section 5.4).

While the results in figure 5.3 are for melanoma, the location of the peak of the specific energy distribution varies somewhat with bulk tissue (results not shown). This shift in peak location is only apparent for incident photon energies $<50 \mathrm{keV}$, where the radiological parameters of the bulk tissues considered herein differ considerably (figure 2.2). For example, melanoma (mammary gland) has relatively high (low) $\mu_{e n} / \rho$ for incident photon energies $<50 \mathrm{keV}$, resulting in fewer (more) MC simulation histories needed to achieve the same $D_{m, m}$. Therefore, there is higher photon fluence in the ROI for mammary gland. Since cellular media are the same in both cases, $f(z, D)$ for mammary gland will be shifted toward higher specific energies for the same $D_{m, m}$.

The specific energy distributions for $\left(r_{\text {cell }}, r_{n u c}\right)=(5,4) \mu \mathrm{m}$ and $(10,4) \mu \mathrm{m}$ shown 

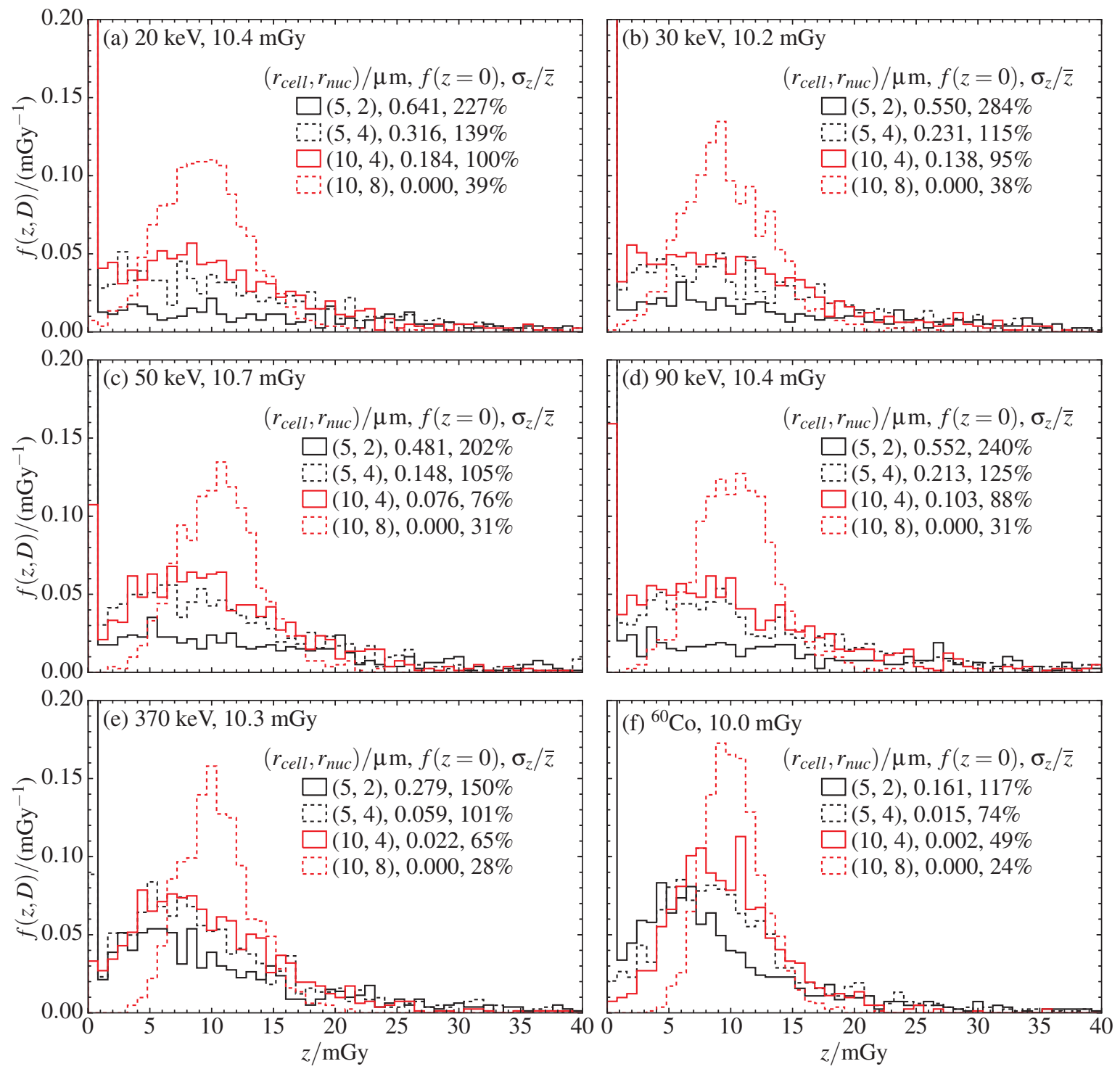

Figure 5.3: Specific energy distributions for nuclear targets for (a) $20 \mathrm{keV}$, (b) $30 \mathrm{keV}$, (c) $50 \mathrm{keV}$, (d) $90 \mathrm{keV}$, (e) $370 \mathrm{keV}$ and (f) ${ }^{60} \mathrm{Co}$ incident photons for melanoma, 'random sizes, random arrangement' (figure 5.1(a)) and $D_{m, m} \simeq 10$ mGy. The fraction of targets receiving no energy deposition and the relative standard deviation of the specific energy are indicated in the legend. The leftmost bin is truncated in some cases. 
in figure 5.3 differ, demonstrating variation in $f(z, D)$ for the same nominal nucleus size but different nominal cell sizes. This discrepancy stems from differences in actual nucleus sizes, as evidenced by the different mean nuclear radii (figure 5.2(a,b)) and volumes (table 5.1). The microdosimetric spread and fraction of nuclei receiving no energy deposition are larger for $\left(r_{\text {cell }}, r_{n u c}\right)=(5,4) \mu \mathrm{m}$, due to its smaller actual mean nucleus size. Furthermore, cytoplasm 'thickness' and the elemental composition/density of the ECM (which varies with cell and nucleus sizes) affect the fluence reaching the nucleus and hence energy deposition therein.

The fraction of nuclear targets receiving no energy deposition is investigated further in figure 5.4. In agreement with trends observed in figure 5.3, $f(z=0)$ for a given $D_{m, m}$ is larger for smaller nucleus sizes and for lower incident photon energies (again, $90 \mathrm{keV}$ is an exception). For the 'random sizes' cases, a larger fraction of nuclei receive no energy deposition when $\left(r_{\text {cell }}, r_{n u c}\right)=(5,4) \mu \mathrm{m}$ compared to $(10,4) \mu \mathrm{m}$. This discrepancy is due to differences in actual nucleus size (as discussed in the previous paragraph), and is therefore not apparent for the 'uniform sizes' cases (results not shown), for which nominal and actual target sizes coincide. For incident photon energies $<50 \mathrm{keV}$, there is some variation in $f(z=0)$ for different bulk tissues for a given $D_{m, m}$, e.g., $f(z=0)$ for mammary gland is generally lower than that of melanoma (see appendix B). As discussed above for figure 5.3, this is due to $\mu_{e n} / \rho$ differences, leading to differences in the number of histories required to achieve a given $D_{m, m}$, and corresponding differences in the photon fluence present within the ROI.

Specific energy distributions for nuclear targets are compared with normal distributions having the same mean and standard deviation in figure 5.5. Agreement between $f(z, D)$ and the corresponding normal distribution is quantified by the RMSE (given in the legend; also see appendix B). For lower doses and smaller target sizes, RMSE values are considerable, $f(z, D)$ is skewed and a considerable fraction of targets receive no energy deposition (i.e., the height of the leftmost bin is considerable). With increasing 

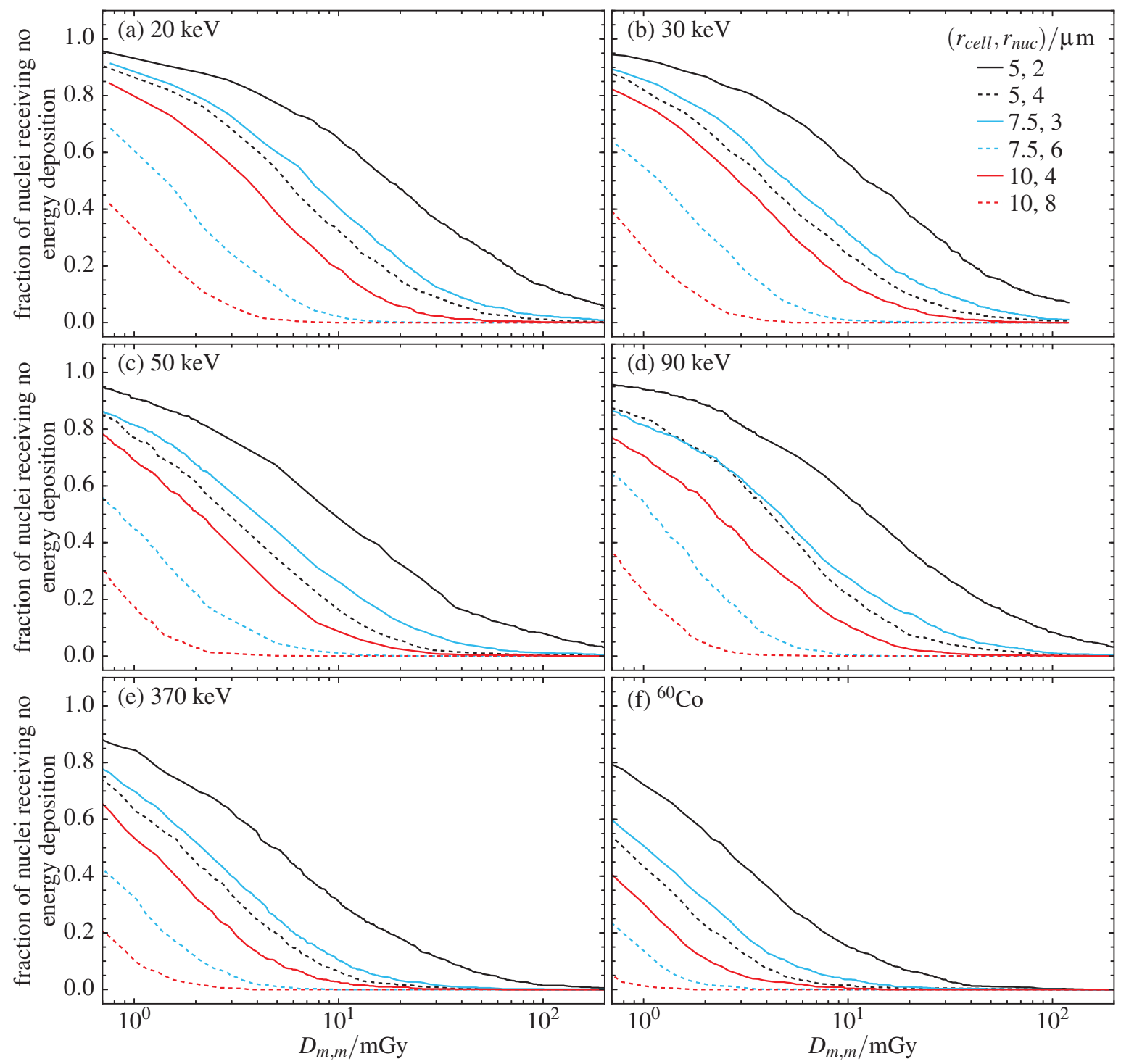

Figure 5.4: Fraction of nuclei receiving no energy deposition as a function of $D_{m, m}$ for (a) $20 \mathrm{keV}$, (b) $30 \mathrm{keV}$, (c) $50 \mathrm{keV}$, (d) $90 \mathrm{keV}$, (e) $370 \mathrm{keV}$ and (f) ${ }^{60} \mathrm{Co}$ incident photons for melanoma and 'random sizes; random arrangement'. 
target size and/or dose, $f(z, D)$ becomes less skewed and the height of the leftmost bin decreases, corresponding RMSE values decrease with $f(z, D)$ approaching normality; the microdosimetric spread decreases as well.
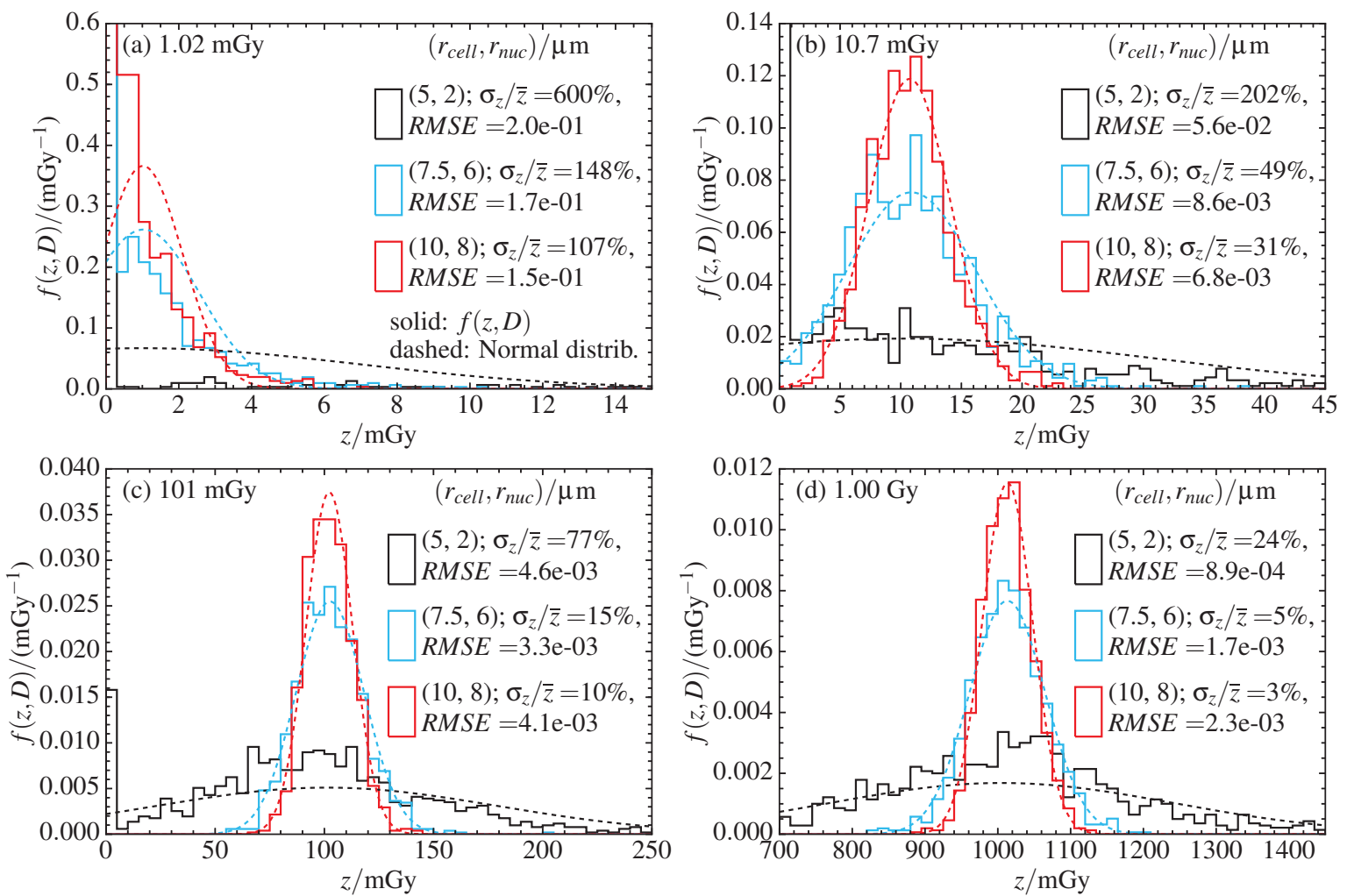

Figure 5.5: Specific energy distributions for nuclear targets (solid lines) are compared with normal distributions (dashed curves; RMSE indicated in legend) having the same mean and standard deviation, for $D_{m, m}$ values of (a) $1.02 \mathrm{mGy}$, (b) $10.7 \mathrm{mGy}$, (c) $101 \mathrm{mGy}$, and (d) 1.00 Gy for melanoma, 'random sizes, random arrangement', and 50 keV incident photons.

In general, mean specific energies for nuclear and cytoplasm targets differ from $D_{m, m}$ (figure 5.6). In addition to the fact that $z$ and $D_{m, m}$ are scored in different media, the presence of explicitly-modelled cells and the surrounding ECM in place of homogeneous bulk tissue affects the fluence present within the ROI. For example, in figure 5.6(e), $\left(r_{\text {cell }}, r_{\text {nuc }}\right)=(10,8) \mu \mathrm{m}$, and electron ranges are comparable to cellular dimensions for incident photon energies below $50 \mathrm{keV}$. As a result, $\bar{z} / D_{m, m}$ is greater than (less than) unity for mammary gland (melanoma) because bulk tissue $\mu_{e n} / \rho$ values 
are lower (higher) than those of cellular media. The influence of cell/nucleus size, choice of cellular and bulk tissue media, and microscopic detail in the surrounding environment (e.g., the ECM and the presence of surrounding cells) has been investigated in chapter 3, which focused on kilovoltage photon energies. Furthermore, the error bars on $\bar{z} / D_{m, m}$ are larger for smaller target sizes, and for lower doses (results not shown).

The relative standard deviation of the specific energy is plotted as a function of $D_{m, m}$ in figure 5.7, illustrating decreases in microdosimetric spread with increasing $D_{m, m}$ as well as variations in $\sigma_{z} / \bar{z}$ with microscopic tissue model (figure 5.1). Results are not strongly dependent on cell arrangement method, but are sensitive to the method used to select subcellular compartment sizes, with the 'random sizes' cases generally resulting in more microdosimetric spread. Some of the variation among curves for different microscopic tissue models can be explained by differences between nominal and actual mean subcellular compartment sizes (table 5.1). The results in figure 5.7 vary somewhat with bulk tissue for incident photon energies $<50 \mathrm{keV}$, e.g., $\sigma_{z} / \bar{z}$ for mammary gland is generally lower than that of melanoma (see appendix B), due to $\mu_{e n} / \rho$ differences (as discussed above for figure 5.3).

Fit parameters for equation 5.2 relating $\sigma_{z} / \bar{z}$ to $D_{m, m}$ and the target volume are presented in table 5.2, along with RMSE values representing the standard deviation of the residual. These parameters are obtained using MC simulation results for the 'random sizes, random arrangement' case (figure 5.1(a)), and the actual mean target volume (table 5.1). If nominal volumes are used instead, then the mean RMSE increases from 0.038 to 0.048 (where the mean RMSE is averaged across all scenarios (e.g., energies, target types) where distinct fit parameters are calculated). If the fitting procedure is carried out for the 'uniform sizes, hexagonal arrangement' case, then the mean RMSE decreases from 0.038 to 0.021 . For the same energy, cytoplasm RMSE values given in table 5.2 are smaller than nucleus RMSE values. For the cell/nucleus sizes considered 

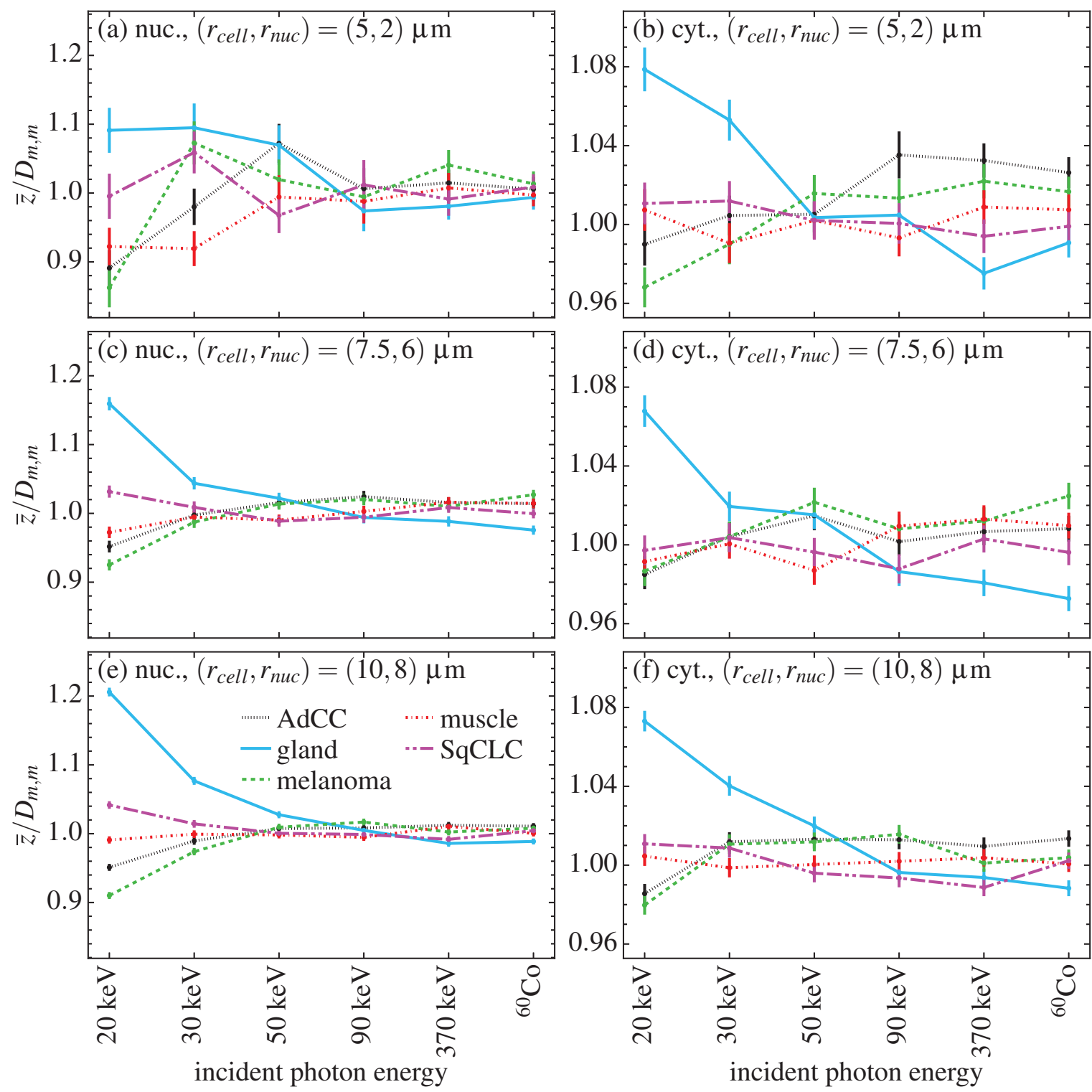

Figure 5.6: Values of $\bar{z} / D_{m, m}$ versus incident photon energy for (a,c,e) nuclear ('nuc.') and (b,d,f) cytoplasm ('cyt.') targets for three cell/nucleus sizes, 'random sizes; random arrangement' and $D_{m, m} \simeq 50 \mathrm{mGy}$. Data points are joined with lines to guide the eye. Error bars are determined from error propagation, with the error on $\bar{z}$ given by $\sigma_{z} / \sqrt{N}$, where $N$ is the cell population size; the error on $D_{m, m}$ is the $1 \sigma \mathrm{MC}$ statistical uncertainty. 

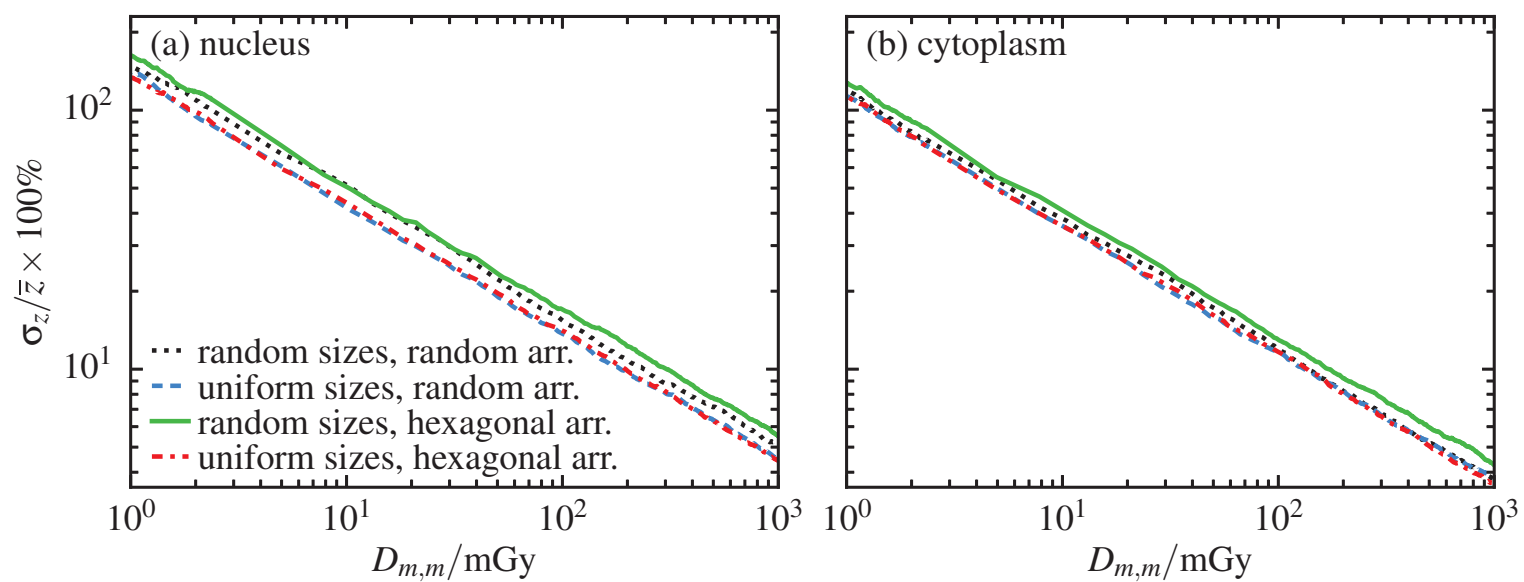

Figure 5.7: The relative standard deviation of the specific energy is plotted as a function of $D_{m, m}$ for (a) nuclear and (b) cytoplasm targets for $\left(r_{\text {cell }}, r_{n u c}\right)=(7.5,6) \mu \mathrm{m}$, for melanoma, for $50 \mathrm{keV}$ incident photons. The legend gives the cell/nucleus size distribution and cell arrangement method.

herein, cytoplasm volumes are generally larger than nuclear volumes; smaller target sizes are more prone to statistical fluctuations, which will generally increase disagreement with equation 5.2.

Table 5.2: Fit parameters for equation 5.2, which relates $\sigma_{z} / \bar{z}$ to $D_{m, m}$ and the target volume, for nucleus and cytoplasm targets. Equation 5.2 is fit to results for the 'random sizes, random arrangement' case (figure 5.1(a)), for all bulk tissues and cell/nucleus sizes, for doses $>10$ mGy. The RMSE is calculated according to equation 5.1.

\begin{tabular}{lcccccccc}
\hline & \multicolumn{3}{c}{ Nucleus } & & \multicolumn{3}{c}{ Cytoplasm } \\
\cline { 2 - 4 } \cline { 6 - 8 } Energy & $C_{1}$ & $C_{2}$ & RMSE & & $C_{1}$ & $C_{2}$ & RMSE \\
\hline $20 \mathrm{keV}$ & 2.95 & 0.593 & 0.0544 & & 1.89 & 0.527 & 0.0170 \\
$30 \mathrm{keV}$ & 2.52 & 0.567 & 0.0606 & & 1.31 & 0.479 & 0.0190 \\
$50 \mathrm{keV}$ & 2.73 & 0.605 & 0.0936 & & 1.47 & 0.510 & 0.0240 \\
$90 \mathrm{keV}$ & 3.54 & 0.638 & 0.0924 & & 1.69 & 0.522 & 0.0188 \\
$370 \mathrm{keV}$ & 1.84 & 0.568 & 0.0351 & & 0.770 & 0.437 & 0.0118 \\
${ }^{60} \mathrm{Co}$ & 1.29 & 0.543 & 0.0248 & & 0.723 & 0.461 & 0.00690 \\
\hline
\end{tabular}

Example discrepancies between $\sigma_{z} / \bar{z}$ computed with these fit parameters and corresponding $\mathrm{MC}$ results are presented in table 5.3 (see also appendix B). Table 5.3 demonstrates varying agreement between the fit and MC results, with discrepancies up 
to $24 \%$ for $\left(r_{\text {cell }}, r_{\text {nuc }}\right)=(7.5,3) \mu \mathrm{m}$ with melanoma, and $30 \mathrm{keV}$ and ${ }^{60}$ Co sources. Similar agreement is observed across the results summarized in appendix B. Table 5.3 and the additional tables provided in appendix B also provide summary results across a wide parameter space for other quantities of interest: $D_{m, m}$ and its statistical uncertainty, $f(z=0)$, the RMSE for $f(z, D)$ compared to a corresponding normal distribution, and MC-calculated $\sigma_{z} / \bar{z}$.

Table 5.3: Summary of results for $\left(r_{\text {cell }}, r_{n u c}\right)=(7.5,3) \mu \mathrm{m}$, melanoma, 'random sizes; random arrangement', for nuclear targets: $D_{m, m}$ and corresponding statistical uncertainty ('unc.'), fraction of nuclei receiving no energy deposition, RMSE for MC-calculated $f(z, D)$ compared to a normal distribution with the same mean and standard deviation, relative standard deviation of the specific energy (MC) and percent discrepancy of this value with the fit according to equation 5.2 with parameters in table 5.2, $\% \Delta_{f i t, M C}=\left[\left(\sigma_{z} / \bar{z}\right)_{f i t}-\left(\sigma_{z} / \bar{z}\right)_{M C}\right] /\left(\sigma_{z} / \bar{z}\right)_{M C} \times 100 \%$. See also appendix B.

\begin{tabular}{|c|c|c|c|c|c|c|}
\hline \multirow{2}{*}{ Energy } & \multicolumn{2}{|c|}{$D_{m, m}$} & \multicolumn{2}{|c|}{$f(z)$} & \multicolumn{2}{|c|}{$\sigma_{z} / \bar{z}$} \\
\hline & $\mathrm{mGy}$ & $\%$ unc. & $f(z=0)$ & RMSE & $\mathrm{MC} \times 100 \%$ & $\% \Delta_{f i t, M C}$ \\
\hline \multirow{5}{*}{$30 \mathrm{keV}$} & 1.2 & 2.1 & 0.84 & 0.17 & 482 & -10 \\
\hline & 5.1 & 1.0 & 0.50 & 0.083 & 213 & -2 \\
\hline & 10.3 & 0.7 & 0.31 & 0.041 & 167 & -13 \\
\hline & 50.1 & 0.3 & 0.047 & 0.0054 & 70 & -5 \\
\hline & 100.0 & 0.2 & 0.012 & 0.0026 & 50 & -7 \\
\hline \multirow{5}{*}{${ }^{60} \mathrm{Co}$} & 1.0 & 3.4 & 0.51 & 0.18 & 355 & -24 \\
\hline & 5.1 & 1.5 & 0.098 & 0.040 & 122 & -2 \\
\hline & 10.2 & 1.1 & 0.036 & 0.019 & 84 & 1 \\
\hline & 51.1 & 0.5 & 0.0019 & 0.0046 & 39 & -3 \\
\hline & 102.0 & 0.3 & 0.00096 & 0.0036 & 27 & -2 \\
\hline
\end{tabular}

\subsection{Discussion}

This is the first work to consider the variation in energy deposition across a population of cells using multiscale MC simulation models of normal and cancerous human soft tissues featuring representative (non-water) media. We demonstrate how the stochastic nature of radiation affects energy deposition within subcellular targets in a variety of scenarios, considering the influence of media, cell arrangement method, and distribution of cell/nucleus sizes within the cell population, for various photon source energies. In 
general, higher doses and larger target sizes correspond to a greater average number of interactions occurring in a target region, resulting in fewer targets receiving no energy deposition (figure 5.4), and less stochastic variation in energy deposition among targets (figures 5.3, 5.6, and 5.7).

Trends in specific energy distributions with source energy, dose level, elemental composition, and subcellular compartment size depend on the relative importance of different photon interactions, the energy transferred in each interaction, secondary electron ranges, scattered photon ranges, and the number of photon interactions in the ROI needed to achieve a particular $D_{m, m}$. Photoelectric interactions dominate at lower photon energies, with photoelectron energies (and therefore ranges) increasing with source energy, in competition with the increasing importance of Compton (i.e., incoherent) interactions. Compton scattering results in lower energy secondary electrons, and scattered photons which tend to escape the ROI (if that is where the interaction occurs); thus, there is potentially a need for more photon interactions in the ROI to achieve a particular $D_{m, m}$. Furthermore, as photon energies increase, photons transfer an increasingly large fraction of their energy to electrons in Compton interactions. ${ }^{83}$ A reduction in the microdosimetric spread and number of targets receiving no energy deposition result from more 'sharing' of energy deposition throughout the target population. The need for more photon interactions in the ROI to achieve a given $D_{m, m}$, and the occurrence of higher energy (longer range) secondary electrons that deposit their energy in multiple targets will both contribute to more 'sharing' (corresponding to reduced $\sigma_{z} / \bar{z}$ and $\left.f(z=0)\right)$. The relative importance of these contributions varies with source energy, target sizes and their distributions, and elemental compositions MC simulations are required to fully understand and predict these trends.

In figures 5.3 and 5.4, it was observed that $\sigma_{z} / \bar{z}$ and $f(z=0)$ generally tend to decrease with increasing incident photon energy, with some exceptions observed at $90 \mathrm{keV}$. This is due to the physics considerations outlined in the preceding paragraph. 
In particular, while photoelectric cross sections decrease relative to those for Compton interactions as photon energy increases, a considerable fraction of the energy deposited in the ROI comes from photoelectric interactions at $50 \mathrm{keV}$. The resulting photoelectrons have considerable range $\left(R_{C S D A} \simeq 43 \mu \mathrm{m}\right)$, thus potentially depositing energy in multiple targets. At $90 \mathrm{keV}$, a much smaller fraction of the energy deposited comes from photoelectrons, and Compton electrons $(<24 \mathrm{keV})$ 'share' less energy among targets within the ROI (noting also different numbers of interactions in the ROI to achieve the same $D_{m, m}$ for 50 and $\left.90 \mathrm{keV}\right)$.

The specific energy distribution, $f(z, D)$, is strongly dependent on cell/nucleus sizes (figure 5.3) and thus also whether these are uniform or sampled from a distribution (figure 5.7). Considering randomly-sampled cell/nucleus sizes to be more realistic, using the same cell/nucleus sizes throughout the cell population (or uniformly-sized voxels, as in Villegas et $\left.a l .{ }^{64}\right)$ will likely lead to underestimation of $\sigma_{z} / \bar{z}$ and $f(z=0)$, which highlights the importance of realistic multicellular models. For example, $\sigma_{z} / \bar{z}$ for nuclear targets is $167 \%$ for 'random sizes, random arrangement' compared to $106 \%$ for 'uniform sizes, hexagonal lattice'; $f(z=0)$ is 0.31 and 0.25 , respectively, considering $30 \mathrm{keV}$ photons incident on melanoma with $\left(r_{\text {cell }}, r_{n u c}\right)=(7.5,3) \mu \mathrm{m}$ for $D_{m, m} \simeq 10 \mathrm{mGy}$. In contrast, results are relatively insensitive to cell arrangement method (figure 5.7).

Previous computational microdosimetry studies (e.g., Villegas et al. ${ }^{64}$ ) focused on all-water models. In contrast, the present work considers representative media, resulting in $\bar{z} \neq D_{m, m}$ (figure 5.6; when $D_{m, m} \simeq 50 \mathrm{mGy}, \bar{z}$ for nuclear (cytoplasm) targets is up to $21 \%(12 \%)$ larger than $\left.D_{m, m}\right)$, which emphasizes the importance of using realistic elemental compositions. A subset of simulations was carried out considering the six other cellular elemental compositions (see table 2.1) (results not shown). If the target medium is changed to one that has relatively large values of $\mu_{e n} / \rho(e . g$. nucleus/cytoplasm elemental composition 'a'), then the number of photon interactions occurring within a target will increase. If electron ranges are small compared to target 
size, then this will result in a corresponding increase in $\bar{z}$. For example, at $20 \mathrm{keV}$, use of cellular elemental composition ' $\mathrm{a}$ ' instead of ' $\mathrm{g}$ ' results in an increase in $\bar{z}$ for nuclear targets for $r_{n u c}=8 \mu \mathrm{m}$, but not for $r_{n u c}=2 \mu \mathrm{m}$. For $r_{n u c}=2 \mu \mathrm{m}$, a considerable portion of energy deposited within the nucleus is due to electrons set into motion outside of the nucleus, and the relatively low values of $S_{\text {col }} / \rho$ for composition 'a' compete with its relatively high $\mu_{e n} / \rho$ values. Previous work has considered in more detail how subcellular compartment sizes and elemental compositions influence energy deposition within subcellular targets resulting from kilovoltage photon irradiation, including consideration of the relation between mean nuclear dose $(\bar{z})$ and bulk tissue dose $\left(D_{m, m}\right)$ (chapters 3 and 4).

Energy deposition within populations of at least $10^{3}$ cells are considered in this chapter, however larger populations might be considered (with additional computing time). For low $D_{m, m}$ and small target sizes, a considerable fraction of nuclei receive no energy deposition (figure 5.4) such that the number of targets contributing a nonzero value to the calculation of the mean or standard deviation of the specific energy is small, and the resulting $\bar{z}$ or $\sigma_{z}$ may be prone to statistical fluctuations. Furthermore, we present results for a single phantom size of $(2 \mathrm{~cm})^{3}$. A subset of simulations was carried out using a $(1 \mathrm{~cm})^{3}$ phantom; for the same $D_{m, m}$, resulting $f(z, D)$ were approximately insensitive to phantom size.

In addition to variation in energy deposition (due to e.g., the stochastic nature of radiation), biological response is also influenced by variation in radiosensitivity throughout a population of cells (due to cells being in different stages of the cell cycle ${ }^{10}$ ), and uncertainty in the radiation source. ${ }^{19}$ During mitosis, the nuclear membrane is dissolved, and the nuclear plasm and cytoplasm mix; the resulting dosimetric impact could be investigated by modelling some cells in the population as spheres containing a homogeneous mixture of nuclear and cytoplasm media. Based on the results presented herein, changing the elemental composition of the target is expected to result in a shift 
in $\bar{z}$. The larger variety of target sizes present within the cell population would likely result in a larger microdosimetric spread (based on the observation that $\sigma_{z} / \bar{z}$ for 'random sizes' cases are larger than for 'uniform sizes' cases — see figure 5.7).

The considerable spread in energy deposition across a population of cells (figure 5.7) implies that some nuclei receive energy deposition that differs considerably from $D_{m, m}$. This result has potentially important implications for radiation risk assessment, given the risk of radiation-induced cancer associated with diagnostic radiology. ${ }^{11}$ In the context of radiotherapy, a small number of surviving cells can proliferate and lead to cancer recurrence. ${ }^{19}$ Furthermore, for low doses and small target sizes, the specific energy distribution is skewed, and does not resemble a normal distribution (figure 5.5). The microdosimetric spread is also considerably larger than the corresponding MC simulation statistical uncertainty in $D_{m, m}$ for the simulation geometries considered herein (table 5.3 and appendix B). For example, $\sigma_{z} / \bar{z}=167 \%$ for nuclear targets, $30 \mathrm{keV}$, melanoma, $\left(r_{\text {cell }}, r_{\text {nuc }}\right)=(7.5,3) \mu \mathrm{m}$, 'random sizes, random arrangement', and $D_{m, m}=10.3 \mathrm{mGy}$. The corresponding uncertainty in $D_{m, m}$ is $0.7 \%$. Also, $31 \%$ of nuclei receive no energy deposition in this case. The relative standard deviation of the specific energy and the fraction of targets receiving no energy deposition are examples of experimentally-relevant quantities that are useful for gauging the importance of microdosimetric considerations, and guiding measurement and data analysis techniques.

In contrast, the requirement that $f(z, D)$ be normally distributed does not necessarily indicate whether or not microdosimetric considerations are necessary. For example, considering the results for $\left(r_{\text {cell }}, r_{n u c}\right)=(5,2) \mu \mathrm{m}$ in figure $5.5(\mathrm{~d})$ : the RMSE is relatively low, but $\sigma_{z} / \bar{z}=24 \%$, which is likely considered a non-negligible amount of variation in most scenarios. The Shapiro-Wilk test for normality ${ }^{137}$ is used by Villegas et al., ${ }^{64}$ and is recommended in the literature. ${ }^{138}$ However, in practical applications, normal distributions may prove to be adequate approximations of $f(z, D)$ to doses lower than the stringent, $\sim 1$ Gy thresholds presented by Villegas et al. ${ }^{64}$ as evidenced by our 
normal distribution comparisons in figure 5.5 (and corresponding RMSE values — see table 5.3 and appendix B), as well as consideration of the fraction of targets receiving no energy deposition (figure 5.4, table 5.3 and appendix B). Ghasemi and Zahediasl ${ }^{138}$ point out that the Shapiro-Wilk test is sensitive to sample size, with 'large' samples often resulting in the null hypothesis that the distribution is normal being rejected even when the deviation from normality is 'small'.

\subsection{Conclusions}

The results of this chapter demonstrate that specific energy distributions for nuclei and cytoplasms are sensitive to incident photon energy, dose level, tissue elemental composition, cell/nucleus size, and cell/nucleus size distribution. For $\sim \mathrm{mGy}$ doses and $\sim \mu \mathrm{m}$ target sizes, $f(z, D)$ for a population of cells can be skewed (i.e., it does not resemble a normal distribution), can have a considerable microdosimetric spread, and can have a considerable number of subcellular targets receiving no energy deposition. Therefore, reporting a single value for the absorbed dose does not fully describe the stochastic nature of energy deposition. Results presented herein and in appendix B may be useful for researchers who wish to gauge the importance of microdosimetric considerations in a particular scenario. Additionally, fit parameters for an equation relating the relative standard deviation of the specific energy to $D_{m, m}$ and the target volume are presented;

this equation provides a convenient means of estimating the microdosimetric spread in a variety of scenarios, without the need to carry out multicellular MC simulations. The pronounced differences between macroscopic (i.e., bulk tissue) dose descriptors and mean specific energies for subcellular targets at low energies are due to strongly medium-dependent radiological parameters and secondary electron ranges that are comparable to cellular length scales, so that fluence and energy deposition are sensitive to the details of the microscopic tissue structure model; this sensitivity underlines the importance of using realistic, multicellular, microscopic tissue structure models. 


\section{Chapter 6}

\section{Microdosimetric considerations for radiation response studies using Raman spectroscopy}

Recent studies have used Raman spectroscopy to investigate cellular response to radiation using microscopic sampling regions. This chapter uses Monte Carlo simulations to investigate how the stochastic nature of radiation affects such measurements. Populations of cells are modelled with sampling volumes located within each nucleus; the effect of averaging over several sampling volumes within the same nucleus is explored. A grid of cubic scoring regions (voxels) are used to investigate variation in the microdosimetric spread as a function of dose and target size. 


\subsection{Introduction}

Raman spectroscopy (RS) involving um-sized sampling volumes is a promising technique for investigating cellular responses to radiation, allowing consideration of subcellular components based on knowledge of different molecular vibration frequencies. This technique has been used to investigate radiation damage to plasma membranes of Chinese hamster cells, ${ }^{20}$ and detect radiation-induced chemical and structural changes in DNA of mouse breast cancer cells. ${ }^{139}$ Lakshmi et $a l .{ }^{21}$ irradiated the heads of mice, observing changes in Raman spectra not only for brain tissue, but also for leg muscle tissue. Harder et al. ${ }^{140}$ pointed out the potential of RS for assessing radiosensitivity, demonstrating changes in glycogen accumulation (potentially indicative of hypoxic recovery) in non-small cell lung cancer (NSCLC) cells in response to $6 \mathrm{MV}$ photon irradiation. Vidyasagar et al. ${ }^{141}$ used RS to predict whether or not cervical tumour shrinkage will occur in response to radiotherapy. RS has also been used to evaluate radiation response in vitro in mammary epithelial cells, ${ }^{142}$ oral cancer cells, ${ }^{143}$ prostate tumour cells, ${ }^{144}$ and breast and lung tumour cells. ${ }^{145}$

The studies cited above involve doses of several Gray, relevant for radiotherapy, however, RS applications include investigation of cell response to low radiation doses $(<1 \mathrm{~Gy})$, relevant for diagnostic radiology or out-of-field radiation in radiotherapy (as well as radiation protection in general). Maguire et al. ${ }^{69}$ demonstrated that RS is capable of detecting ${ }^{60} \mathrm{Co}$ radiation response in human lymphocytes for doses as low as 0.05 Gy; DNA damage predicted by changes in Raman spectra were confirmed by comparison with $\gamma$-H2AX assay results. Allen et $a .^{70}$ irradiated human lens epithelial (HLE) cells with $120 \mathrm{kVp}$ x-rays to doses as low as $0.01 \mathrm{~Gy}$, finding that Raman band intensities for various proteins and nucleic acids in the nucleus exhibit non-linear behaviour as a function of dose below 0.5 Gy; they noted that the corresponding cell 
survival curve demonstrates a similar shape (indicative of a region of low dose hypersensitivity followed by a region of radio-resistance). Meade et al. ${ }^{146}$ used RS to observe the same dose response trends in human keratinocyte (HaCaT) cells exposed to ${ }^{60} \mathrm{Co}$ radiation, considering doses as low as 0.005 Gy (in addition to investigations of the bystander effect).

Despite the recent progress in applying $\mathrm{RS}$ to probe radiation response on microscopic (cellular) levels, studies have relied on the macroscopic dose to quantify the amount of energy deposited without considering the stochastic nature of radiation transport and energy deposition. For low doses and/or small targets, there can be considerable variation in specific energy deposited within a population of targets. Variation in energy deposition on microscopic scales (microdosimetric spread), may lead to variation in biological response. ${ }^{15,17}$ This radiation-induced biological response is studied via analysis of the measured RS signal. Thus, with studies employing $\mu$ m-sized sampling volumes and a wide range of doses (as low as 0.005 Gy), microdosimetric considerations may be of importance for radiation response studies using RS. This provides the context and motivation for the current chapter. Specific energy distributions are investigated using MC simulation geometries relevant for $\mathrm{RS}$ cellular radiation response studies. Our study has two parts: in part 1, MC simulations are carried out within a multicellular model; two types of RS sampling volumes, which are located within each cell nucleus, are considered (motivated by recent studies). We investigate specific energy distributions for nuclei and sampling volumes. In part 2, we consider variation in energy deposition across a population of cubic voxels as a function of dose and target size, with the goal of presenting results over a wider range of target sizes than in part 1 as well as providing reference data for future RS experiments. 


\subsection{Methods}

The EGSnrc user-code egs_chamber, ${ }^{28,72}$ along with the EGSnrc C++ class library ${ }^{73}$ is used to score specific energy in nuclei and sampling volumes in part 1, and in voxels in part 2. As in the previous chapter, a version of egs_chamber that is modified to allow scoring energy deposition in multiple regions is used.

Three incident photon spectra are considered: a $120 \mathrm{kVp}$ x-ray spectrum, a cobalt60 spectrum, and a $6 \mathrm{MV}$ medical linac photon spectrum. The $120 \mathrm{kVp}$ spectrum corresponds to an x-ray source with Tungsten target and $2 \mathrm{~mm}$ of aluminum filtration, and is obtained from the Siemens x-ray spectra simulation tool, ${ }^{147}$ which can be accessed online (https://www.oem-xray-components.siemens.com/x-ray-spectra-simulation). The ${ }^{60}$ Co photon spectrum is from Rogers et al. ${ }^{135}$ The $6 \mathrm{MV}$ photon spectrum is from Mohan et al. ${ }^{148}$ A parallel photon beam with circular cross section is oriented along the $z$-axis.

The multicellular model used in part 1 (figure 6.1) is comprised of 1600 cells arranged in a $40 \times 40$ grid in the $x y$-plane located at the centre of a cubic phantom; all media are set to unit density water. Cells and nuclei are modelled as cylinders whose height and diameter are equal, with radii (denoted $r_{\text {cell }}$ and $r_{n u c}$, respectively) from measurements of human stage III NSCLC cells carried out by Lee et al.: ${ }^{103} r_{\text {cell }}=9.2 \mu \mathrm{m}$ and $r_{n u c}=6.4 \mu \mathrm{m}$. The minimum cell separation distance is $2.06 \mu \mathrm{m} .{ }^{35}$ Two sizes of sampling volumes within the nucleus are considered: a 'small' cylinder with 1 um diameter and $4 \mathrm{~mm}$ height, ${ }^{70}$ and a 'large' rectangular prism measuring $2 \times 5 \times 10 \mathrm{\mu m}^{3} .{ }^{140}$ Nine of the small sampling volumes are located in the nucleus of each cell (figure 6.1(a)); the small sampling volumes are arranged in a $3 \times 3$ grid in the $x y$-plane, with $3 \mu \mathrm{m}$ separating the centres of adjacent sampling volumes (as in Allen et al. ${ }^{70}$ ). Only a single large sampling volume is simulated at the centre of each nucleus (figure 6.1(b)). Specific energy is scored in the nucleus of each cell, and in all small and large sampling 


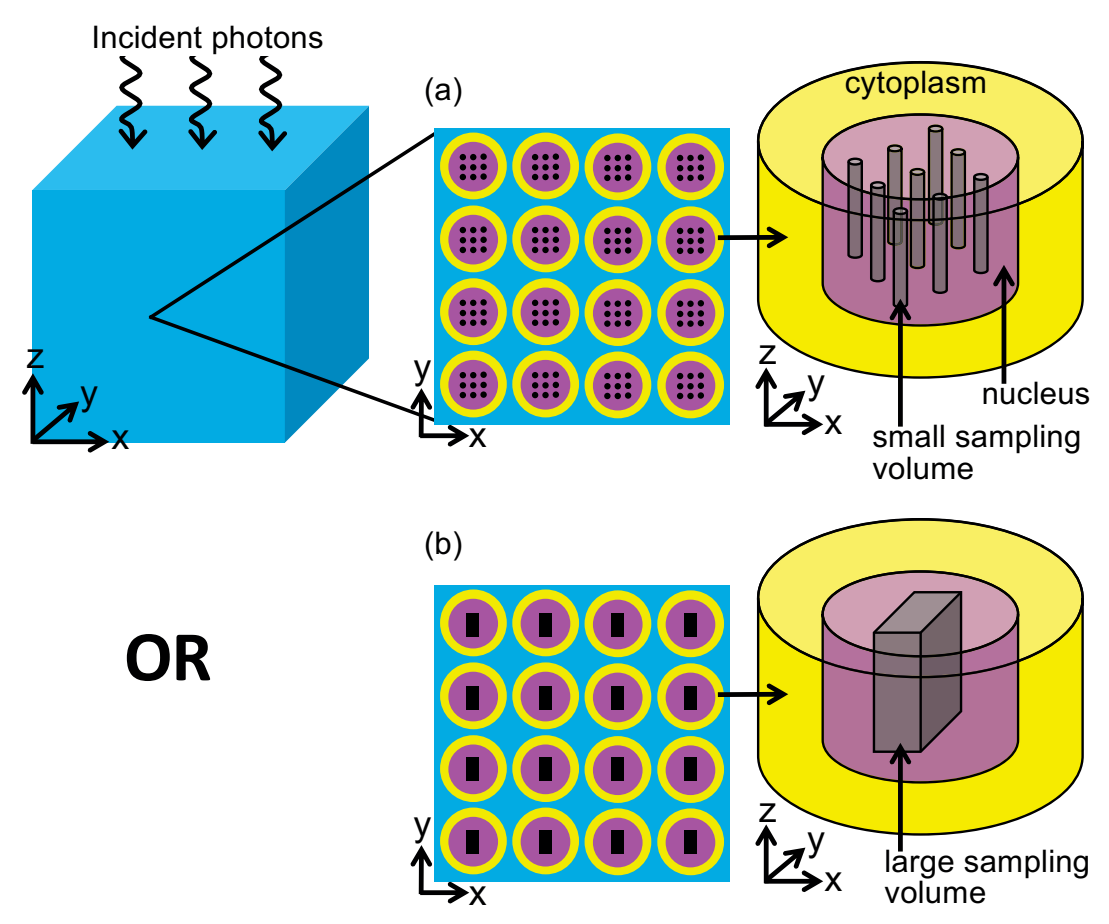

Figure 6.1: Schematic diagrams of simulation geometries used in part 1: (a) cells with nine 'small' sampling volumes, (b) cells with one 'large' sampling volume. Unit density water is used throughout; different colours are used to distinguish between different regions. A $40 \times 40$ grid of cells in the $x y$-plane is located at the centre of the cubic phantom shown on the left side of the figure; only a subset of cells are shown, for clarity. Diagrams are not to scale.

volumes. In addition to considering each small sampling volume independently, we also investigate the effect of averaging the specific energy across the nine sampling volumes located within each nucleus.

In part 2 , a $40 \times 40$ grid of cubic voxels located in the $x y$-plane is considered. As with the multicellular model considered in part 1, voxels are located at the centre of a cubic phantom, and all regions contain unit density water. Voxel side lengths of 1, 3, 5, 7, 9 and $11 \mu \mathrm{m}$ are considered. Specific energy is scored in each voxel.

To balance the need for adequate buildup/scatter with simulation efficiency, cubic phantom side lengths and beam radii are chosen to vary depending on the incident photon spectrum. The phantom side length is roughly 6 times the (electron) $R_{C S D A}$. 
Therefore, the cell or voxel populations, which are located at the centres of the phantoms, are at a depth of $\sim 3 R_{C S D A}$. For the $120 \mathrm{kVp}$ x-ray spectrum, the maximum energy of $120 \mathrm{keV}$ is used to calculate $R_{C S D A}$, and we round up to $1 \mathrm{~cm}$. For the ${ }^{60} \mathrm{Co}$ and $6 \mathrm{MV}$ spectra, the mean spectrum energies (1.25 and $2.02 \mathrm{MeV}$, respectively) are used. The beam radius is chosen to be $1 / 4$ of the phantom side length. For $120 \mathrm{kVp}$, ${ }^{60} \mathrm{Co}$ and $6 \mathrm{MV}$, this corresponds to phantom side lengths of 1,3 and $6 \mathrm{~cm}$, respectively; beam radii are $0.25,0.75$ and $1.5 \mathrm{~cm}$, respectively.

From the specific energies, $z$, scored via MC simulation in each population of targets (nuclei, sampling volumes, or voxels), we calculate the mean, $\bar{z}$, standard deviation, $\sigma_{z}$, normalized distribution, $f(z, D)$ (with $\int_{0}^{\infty} f(z, D) d z=1$ ), and fraction of targets receiving no energy deposition, $f_{z=0}$. These quantities depend on the corresponding dose, $D$, to a macroscopic region that encompasses the targets where $z$ is scored. In part $1, D$ is scored in a $820.5 \times 820.5 \times 18.5 \mu^{3}$ rectangular prism encompassing the cells. In part $2, D$ is the dose to the entire region where cubic voxels are located. Doses of up to 2 Gy are considered. MC simulations are split up across many CPUs. To obtain results for a particular dose $D$, results from different CPUs are added up, such that there is limited dose resolution. The actual dose is indicated in most cases; otherwise, an approximate value is given.

In part 2, specific energy distributions for cubic voxel are compared with normal distributions having the same mean and standard deviation, and the root mean square error is calculated as follows:

$$
\mathrm{RMSE}=\sqrt{\frac{1}{N}\left[\sum_{i=1}^{N}\left(f_{i, \text { normal }}-f_{i, M C}\right)^{2}\right]}
$$

where $f_{i, \text { normal }}$ and $f_{i, M C}$ are $f(z, D)$ values according to the normal distribution (evaluated at the bin centre) and MC simulation, respectively. $N$ is the number of bins in the $f(z, D)$ histogram; a bin width of $1 \mathrm{mGy}$ is used to calculate the RMSE, with 
bins spanning the range within three standard deviations of the mean (the range will start at zero if the mean less three standard deviations is a negative number). We also investigate the relationship between the microdosimetric spread, dose level and target volume using the following equation, which is also used in chapter 5:

$$
\sigma_{z} / \bar{z}=\frac{C_{1}}{V^{C_{2}} \sqrt{D}}
$$

where $\sigma_{z} / \bar{z}$ is the relative standard deviation of the specific energy (i.e., the microdosimetric spread), $C_{1}$ and $C_{2}$ are unknown constants, $V$ is the target volume in $\mu^{3}$, and $D$ is in Gy. The fit is performed by combining data across all cubic voxel sizes, and across the full range of available doses (up to 2 Gy) for each incident photon spectrum. The goodness of fit of equation 6.2 is evaluated via the RMSE; in this case, the sum in equation 6.1 is over the entire parameter space consisting of all target sizes, and available doses. The quantities $f_{i, n o r m a l}$ and $f_{i, M C}$ are replaced with $\left(\sigma_{z} / \bar{z}\right)_{i, f i t}$ and $\left(\sigma_{z} / \bar{z}\right)_{i, M C}$, which are the values (for scenario $i$ ) of $\sigma_{z} / \bar{z}$ according to equation 6.2 and MC simulation, respectively.

\subsection{Results}

\subsubsection{Part 1: multicellular models}

Specific energy distributions for different targets are presented in figure 6.2 for various incident photon spectra and dose levels. These distributions, as well as the microdosimetric spread, $\sigma_{z} / \bar{z}$, and fraction of targets receiving no energy deposition, $f_{z=0}$ (both indicated in the figure legend), vary considerably depending on whether the nucleus or different sampling volumes are considered the targets. In general, the distributions are observed to become narrower and less skewed for higher incident photon energies, higher doses, and for larger target sizes. Of all the $f(z, D)$ shown in figure 6.2 , those for

the nucleus (largest target volume, $\sim 1600 \mu \mathrm{m}^{3}$ ) are generally the least skewed with the 
smallest $\sigma_{z} / \bar{z}$ and $f_{z=0}$ for a given dose and source spectrum. In contrast, the $f(z, D)$ for the small sampling volume $\left(\sim 3 \mu \mathrm{m}^{3}\right)$ are the most skewed and have the largest $\sigma_{z} / \bar{z}$ and $f_{z=0}$ for a given dose and source spectrum. Specific energy distributions are more comparable for the large sampling volume $\left(100 \mu^{3}\right)$ and considering the average of the 9 small sampling volumes (total volume $\sim 28 \mathrm{\mu m}^{3}$ ) (corresponding values of $\sigma_{z} / \bar{z}$ and $f_{z=0}$ are also comparable).

The stochastic nature of radiation is especially apparent for lower doses, smaller target sizes and lower incident photon energies - see e.g., figure 6.2(a) (120 kVp source, $D \simeq 0.02 \mathrm{~Gy})$, where $\sigma_{z} / \bar{z}=258 \%$ and $f_{z=0}=0.73$ for the small sampling volume. In contrast, in figure 6.2(i) (6 MV source, $D \simeq 2 \mathrm{~Gy}), f(z, D)$ for the small sampling volume is still slightly skewed, and the relative standard deviation of the specific energy is $17 \%$, although all of the targets receive some amount of energy deposition in this case (i.e., $\left.f_{z=0}=0\right)$. For the source energies and dose levels considered in figure 6.2, the nuclear targets (the largest of the targets considered herein) always receive some amount of energy deposition.

\subsubsection{Part 2: cubic voxel geometries}

Results for cubic voxel geometries are presented in this section, considering results for a wider range of target sizes than in part 1, and providing reference data for researchers including fits to analytic expressions.

Figure 6.3 demonstrates that the microdosimetric spread decreases with increasing dose and target size. At higher doses, more energy deposition events occur within each target. Similarly, for the same dose level, more energy deposition events will occur within a larger target. In both cases, the total specific energy is averaged over a larger number of events, resulting in less stochastic variation in specific energy. Figure 6.3 also demonstrates that the microdosimetric spread decreases with increasing photon energy. 

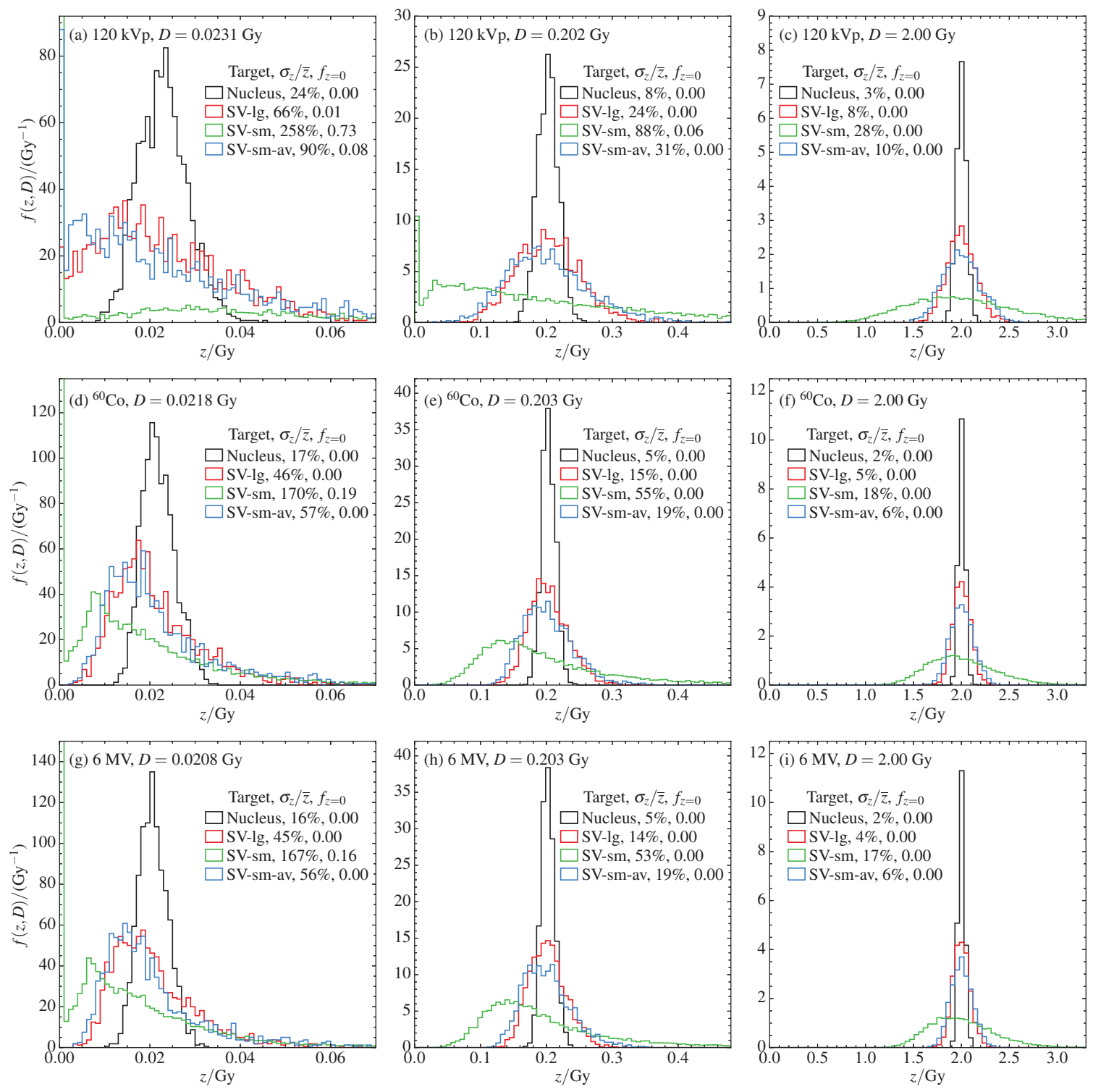

Figure 6.2: Specific energy distributions for nuclear targets, and small and large sampling volumes ('SV-sm' and 'SV-lg', respectively) in multicellular models; 'SV-sm-av' data points come from the average specific energy of nine small sampling volumes within the same nucleus. Source energies are (a-c) $120 \mathrm{kVp}$, (d-f) ${ }^{60} \mathrm{Co}$ and (g-i) $6 \mathrm{MV}$ spectra for $D \simeq 0.02$ Gy $(\mathrm{a}, \mathrm{d}, \mathrm{g}), 0.2 \mathrm{~Gy}(\mathrm{~b}, \mathrm{e}, \mathrm{h})$, and $2 \mathrm{~Gy}(\mathrm{c}, \mathrm{f}, \mathrm{i})$. The relative standard deviation of the specific energy and fraction of targets receiving no energy deposition are given in the legend. 
For all of the photon spectra considered herein, Compton scattering is the dominant photon interaction type. As incident photon energy increases, photons transfer an increasingly large fraction of their energy to Compton electrons, ${ }^{83}$ resulting in longer range secondary electrons that can 'share' their energy among several targets, resulting in less variation in specific energy among targets.
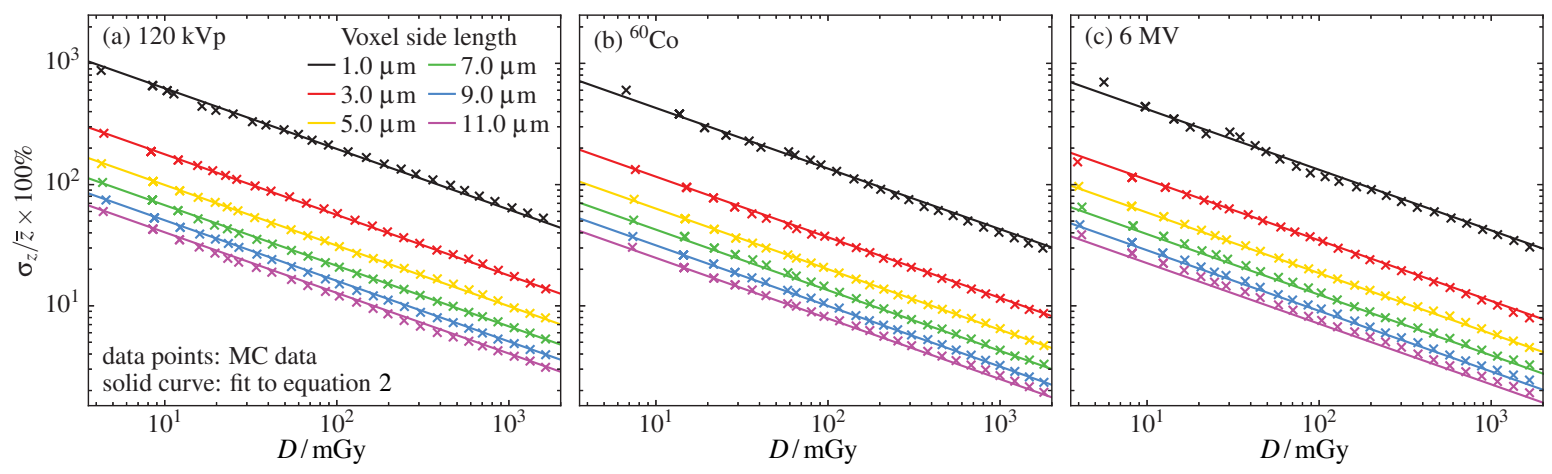

Figure 6.3: Relative standard deviation of the specific energy across a population of cubic voxels (six sizes are considered) as a function of dose for (a) $120 \mathrm{kVp}$, (b) ${ }^{60} \mathrm{Co}$ and (c) $6 \mathrm{MV}$ spectra. Only a subset of MC data points (' $\mathrm{x}$ ' symbols) are shown. The fit (solid curve) is according to equation 6.2 .

Fit parameters for equation 6.2 are presented in table 6.1 ; the fit is shown along with MC-calculated values of $\sigma_{z} / \bar{z}$ in figure 6.3. RMSE values in table 6.1 are similar across the three photon spectra considered herein. Additional numerical values of various quantities of interest are given in table 6.2 to supplement the graphical data provided herein. Estimates of the microdosimetric spread obtained from equation 6.2 agree with $\mathrm{MC}$ results within $10 \%$ for the parameter space considered in table 6.2. Furthermore, for the target sizes investigated herein, the statistical uncertainty on $D$ from MC simulations is much smaller than the microdosimetric spread, $\sigma_{z} / \bar{z}$.

Specific energy distributions for cubic voxel geometries, and corresponding normal distributions having the same mean and standard deviation are presented in figure 6.4 for a subset of voxel sizes and a few doses. For low doses and smaller voxels, $f(z, D)$ are skewed, with a considerable number of voxels receiving no energy deposition and 
Table 6.1: Fit parameters for equation 6.2 relating the relative standard deviation of the specific energy $\left(\sigma_{z} / \bar{z}\right)$ to the target volume and dose. Equation 6.2 is fit to the full range of cubic voxel sizes, and doses up to 2 Gy. The RMSE quantifies the differences between equation 6.2 and $\mathrm{MC}$ simulation results (see section 6.2).

\begin{tabular}{cccc}
\hline Radiation source & $C_{1}$ & $C_{2}$ & RMSE \\
\hline $120 \mathrm{kVp}$ & 0.622 & 0.380 & 0.0265 \\
${ }^{60} \mathrm{Co}$ & 0.429 & 0.396 & 0.0232 \\
$6 \mathrm{MV}$ & 0.419 & 0.407 & 0.0310 \\
\hline
\end{tabular}

considerable microdosimetric spread, in agreement with results from part 1 . The RMSE for MC-calculated $f(z, D)$ compared to the corresponding normal distribution (table 6.2) gives a relative measure of how well the corresponding normal distribution describes the MC-computed $f(z, D)$ for different doses and voxel sizes, demonstrating improved agreement with increasing dose and volume; smaller RMSE values also correspond to smaller $f_{z=0}$ and $\sigma_{z} / \bar{z}$. 

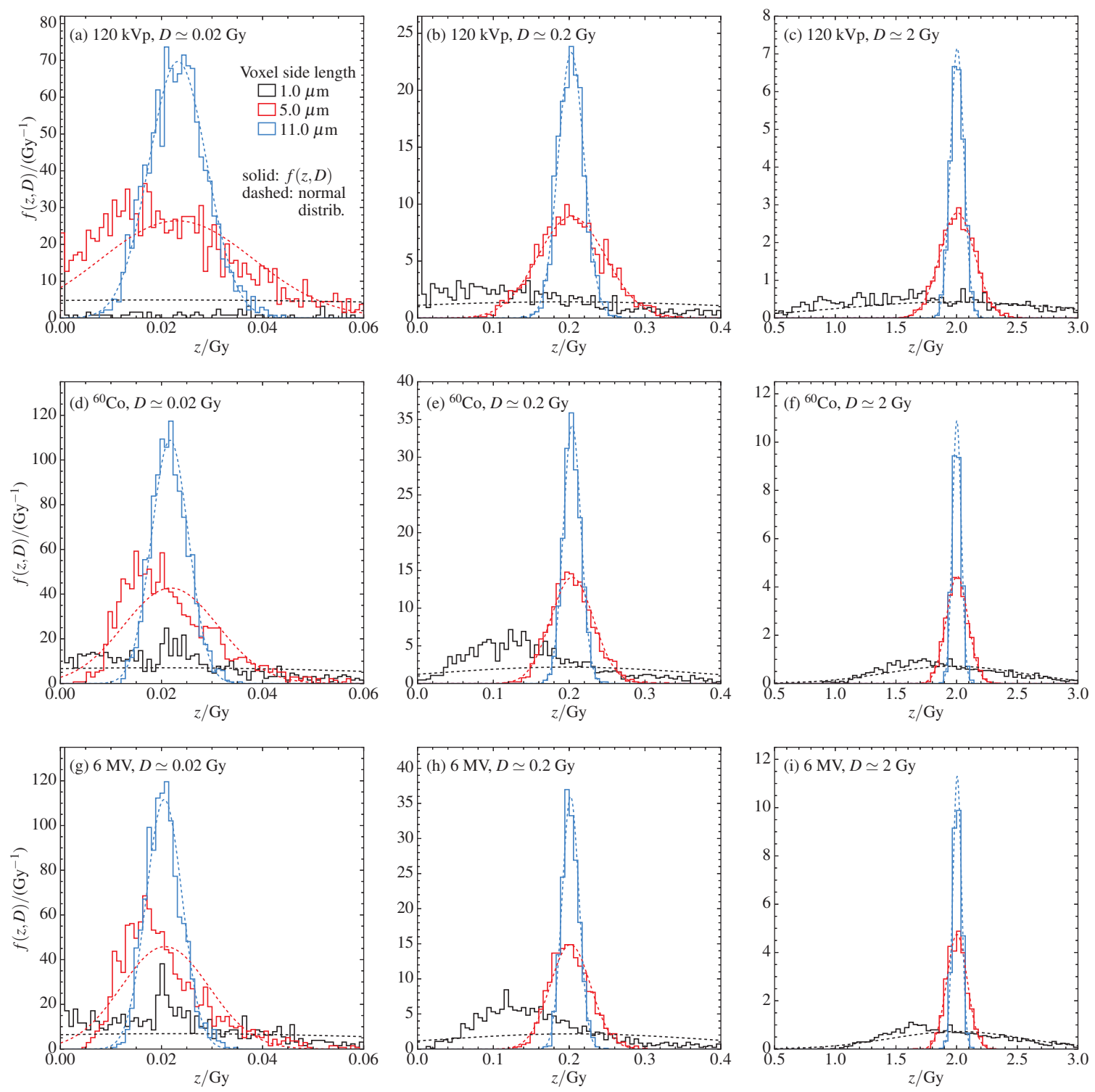

Figure 6.4: Specific energy distributions (solid lines) for populations of cubic voxels are compared with normal distributions (dashed curves) having the same mean and standard deviation for (a,b,c) $120 \mathrm{kVp},(\mathrm{d}, \mathrm{e}, \mathrm{f}){ }^{60} \mathrm{Co}$ and $(\mathrm{g}, \mathrm{h}, \mathrm{i}) 6 \mathrm{MV}$ spectra; dose levels: (a,d,g) $\sim 0.02$ Gy (actual range: 0.0205-0.0255 Gy), (b,e,h) $\sim 0.2$ Gy (actual range: 0.201$0.204 \mathrm{~Gy}$ ), and (c,f,i) $\sim 2$ Gy (actual range: 2.00-2.01 Gy). Three voxel side lengths are considered. The leftmost bin is truncated in some cases. 


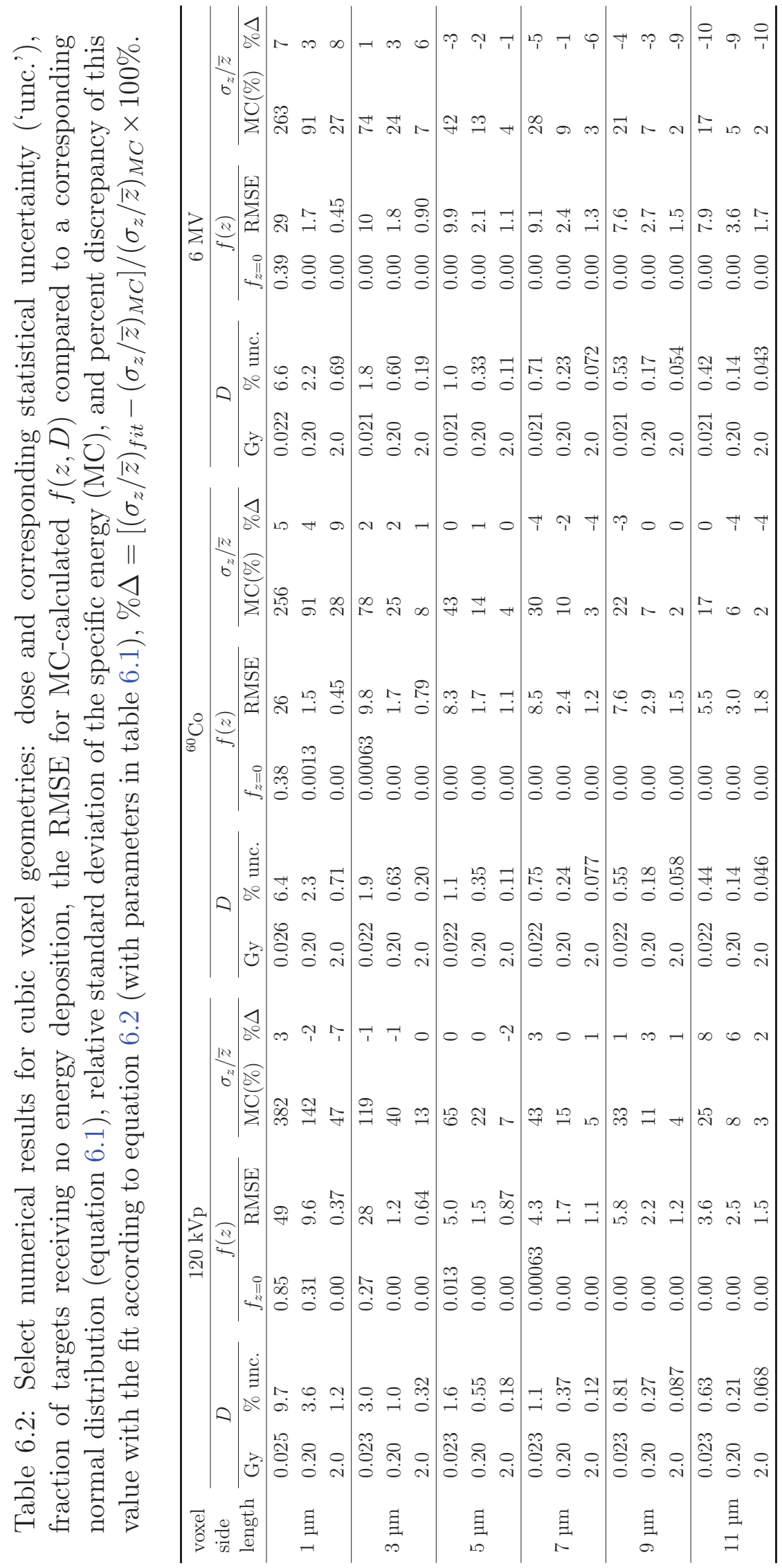




\subsection{Discussion}

There are considerable differences in specific energy distributions, $f(z, D)$, for different target volumes representative of sampling volumes used in recent RS studies (part 1, figure 6.2). These results demonstrate that $f(z, D)$ differs considerably for single small sampling volumes, single large sampling volumes, and for the case where $z$ is averaged across nine small sampling volumes located within the same nucleus; furthermore, these differ from $f(z, D)$ for the nucleus. There are considerable differences in the fraction of targets receiving no energy deposition, the microdosimetric spread, as well as each distribution's skewness and/or shape. Thus, the choice of sampling volume and/or averaging technique may be of importance for RS studies.

Building on these general observations based on MC results from part 1, estimates of microdosimetric spread for some example studies from the recent literature may be obtained using equation 6.2 with fit parameters from part 2 (table 6.1). Allen et $a l{ }^{70}$ used RS sampling volumes equivalent to the small sampling regions considered herein, irradiating HLE cells using a $120 \mathrm{kVp}$ x-ray spectrum and considering doses between 0.01 and 5 Gy. In this case, the microdosimetric spread is $403 \%$ at 0.01 Gy, decreasing to $18 \%$ at 5 Gy. However, Allen et al. averaged Raman spectra across the nine sampling volumes located within each nucleus/cytoplasm, leading to a reduction in $\sigma_{z} / \bar{z}$ (figure 6.2). Maguire et al. ${ }^{69}$ used a $4 \times 4 \mu^{2} \mathrm{RS}$ sampling area for human lymphocyte cells irradiated with a ${ }^{60} \mathrm{Co}$ beam, considering doses of 0.05 and 0.5 Gy. Taking the third dimension of the sampling volume to be equal to the other two, $\sigma_{z} / \bar{z}=$ $37 \%$ and $12 \%$, for 0.05 and 0.5 Gy, respectively. Harder et al. ${ }^{140}$ irradiated human NSCLC cells with a $6 \mathrm{MV}$ linac photon beam, considering doses of 5 and $15 \mathrm{~Gy}$, and using a $2 \times 5 \times 10 \mathrm{\mu m}^{3}$ sampling volume (equivalent to the large sampling volume considered in part 1). Since Harder et al. considered higher doses than the other two examples given in this paragraph, the relative standard deviation of the specific 
energy is smaller in this case: $\sigma_{z} / \bar{z}=3 \%$ and $2 \%$ for 5 and 15 Gy, respectively. These examples demonstrate the considerable variation in microdosimetric spread depending on target/sampling volume (see also figure 6.3). Furthermore, for lower doses and smaller targets/sampling volumes, specific energy distributions may be skewed with a considerable number of targets receiving no energy deposition (figure 6.4 and table 6.2).

The considerable variation in energy deposition among microscopic targets for low doses and/or small sampling volumes demonstrated in section 6.3 can motivate measurement and analysis approaches for future RS investigations. For example, researchers considering microscopic targets and low doses need to acquire a sufficient number of measurements to ensure that the distribution of measured values captures the true, underlying distribution. The measurement technique likely also depends on the identity of the target of interest, e.g., the sampling region should ideally cover the nucleus if that is the target of interest; signal variation among RS measurements should ideally be representative of the true variation in radiation response among nuclei in the cell population or tissue sample. As another example, if a particular cell signalling pathway is of interest, then the relevant biological length scale of that pathway may determine the sampling volume. For high enough doses or large enough target sizes (see e.g., figure 6.2(i), considering $f(z, D)$ for the large sampling volumes), the microdosimetric spread is relatively small; however, a sufficiently large number of measurements is nonetheless needed to account for variation resulting from sources other than the stochastic nature of radiation (e.g., biological effects). Furthermore, discarding outliers ${ }^{70,140}$ should be done with caution; outliers could be due to the stochastic nature of radiation transport and energy deposition, leading to corresponding variation in cell response.

The results of this chapter motivate future research comparing experimental RS measurements with MC simulation results. Such research would potentially lead to a better understanding of the magnitudes of the various contributions to the variation in radiation response throughout a population of biological targets. This application of 
RS has not yet been considered in the literature. In addition to the stochastic nature of radiation, biological response is also determined by e.g., variation in radiosensitivity with cell cycle stage, ${ }^{10}$ and bystander effects. ${ }^{22}$ Furthermore, uncertainty in the experimental setup (e.g., the radiation source, and measurement equipment) can also contribute to variation in the RS signal. Future research could also include more sophisticated cell irradiation techniques: microbeam irradiation and fluorescent nuclear track detectors (FNTDs) are two examples of techniques that can eliminate some of the stochastic uncertainty associated with radiation transport and energy deposition (see chapter 1).

As different sampling volumes or target shapes are employed in different parts of this chapter (cylinders and rectangular prisms in part 1; cubic voxels in part 2 for simplicity), additional simulations are carried out to assess sensitivity to target shape. To determine how cubic voxels relate to a more realistic cell shape such as a sphere, additional simulations were carried out comparing $f(z, D)$ for spherical targets (radii between 2 and $8 \mu \mathrm{m})$ with $f(z, D)$ for volume-matched or mean chord length-matched cubic targets. The mean chord length (MCL) for a convex cavity is calculated as $4 \mathrm{~V} / \mathrm{S}$ for volume $V$ and surface area $S .{ }^{122}$ For $D=10 \mathrm{mGy}$, considering $20 \mathrm{keV}, 50 \mathrm{keV}$ and ${ }^{60} \mathrm{Co}$ incident photons, volume-matched cubic voxels yielded $f(z, D)$ in approximate agreement with $f(z, D)$ for spherical targets, while the use of MCL-matched cubic voxels resulted in the microdosimetric spread being underestimated (results not shown). Therefore, if MC simulations are carried out with a grid of cubic voxels, which is often more convenient to simulate than spheres, then voxel side lengths should be chosen such that the voxel volume is equal to that of the target of interest. Finally, $\sigma_{z} / \bar{z}$ calculated with our fit parameters (table 5.2, for cubic voxels) and those of Villegas et $a l .{ }^{65}$ (determined for spheres) generally differ by about $\sim 10 \%$, likely due to the different calculation methodologies. Furthermore, Villegas et al. use a monoenergetic $1.25 \mathrm{MeV}$ source instead of a full ${ }^{60}$ Co spectrum. 
Equation 6.2 (corresponding fit parameters in table 6.1) provides microdosimetric spread estimates that may be useful for researchers in the field of RS. Additionally, table 6.2 provides numerical results for various quantities of interest. However, results presented in chapter 5 demonstrated that randomly sampling cell and nucleus sizes from a normal distribution will lead to a larger microdosimetric spread than if the cells are modelled in a less realistic manner, with cell and nucleus sizes constant throughout the cell population, as in this chapter. Also, this chapter considers all-water simulation geometries. However, the use of representative, non-water media will affect $f(z, D)$, especially for low $(<50 \mathrm{keV})$ incident photon energies (see chapter 5$)$.

\subsection{Conclusions}

The MC simulation results presented in this chapter demonstrate that there can be considerable variation in energy deposition (microdosimetric spread) throughout populations of $\mu \mathrm{m}$-sized targets relevant for RS studies of cellular response to radiation. The microdosimetric spread is observed to increase with decreasing (macroscopic) absorbed dose, target size, and incident photon energy. Results highlight the importance of microdosimetric considerations (i.e., considering the stochastic nature of radiation transport and energy deposition) for studies using microscopic sampling volumes and low doses. Fit parameters for an equation relating the relative standard deviation of the specific energy to the target volume and macroscopic dose are presented for the three photon source spectra considered herein. Graphical and numerical results are also provided as a guide for researchers in the field of RS. The results of this chapter may motivate changes to current RS measurement and data analysis methods: e.g., choice of number of measurements, size of sampling region, and signal averaging method. Results presented herein also motivate future research using both MC simulation and RS techniques as tools for investigating the various contributions to variation in radiation response throughout a population of biological targets. 


\section{Chapter 7}

\section{Investigating energy deposition in glandular tissues for mammography using multiscale Monte Carlo simulations}

Monte Carlo simulations involving multiscale models of compressed breasts are used to investigate energy deposition in mammary epithelial cell nuclei, and in the macroscopic tissue structure (considering glandular and adipose tissues) for a range of typical mammographic dose levels. Sensitivity to various macro- and microscopic model parameters is explored. 


\subsection{Introduction}

Mammography risk assessment is based on estimations of dose to glandular tissue, which is more radiosensitive than adipose tissue or skin; irradiation of glandular tissue can lead to breast cancer. ${ }^{149}$ Historically, breast dosimetry studies for mammography assumed that breast tissue was a homogeneous mixture of glandular and adipose tissues, considering mean glandular dose (MGD; averaged over the entire breast) as the relevant risk assessment metric. ${ }^{150,151}$ MGD varies with compressed breast thickness, the amount of glandular tissue in the breast (i.e., the glandularity), and source spectrum. ${ }^{152}$ MGD is also dependent on imaging technique (e.g., digital radiography, computed radiography or film-screen system), with the replacement of film-screen imaging with digital radiography resulting in a $\sim 25 \%$ decrease in MGD; typical MGD values range between $\sim 0.5$ mGy and $\sim 7$ mGy. ${ }^{153}$

More recently, MC simulations involving computational phantoms with segmented breast tissue have demonstrated that the use of homogeneous breast tissue models can result in an overestimation of glandular tissue dose by $\sim 30 \%$. This discrepancy is due to glandular tissue being typically more concentrated near the centre of the breast, where dose is lower due to attenuation. ${ }^{154-156}$ The advent of dedicated breast computed tomography (DBCT) provides the opportunity for patient-specific MGD estimates based on a realistic segmented breast tissue model. ${ }^{149}$ Because these DBCT images are often obtained with the breast in a pendant geometry, algorithms for simulated compression have been developed. ${ }^{157}$ Real breast specimens can be compressed prior to CT imaging: ${ }^{158}$ however, this process is likely inconvenient for a live patient. Hernandez et al. ${ }^{155}$ use a database consisting of 219 DBCT images to develop mathematical models describing glandular tissue distributions, which are a function of the radial distance from the centre of mass of the breast. Despite these advances in computational breast modelling, 
a single metric such as the MGD remains the primary focus of mammography breast dosimetry studies.

This chapter investigates energy deposition in glandular tissues in the context of mammography using novel, multiscale models of compressed breasts. Although previous studies have used segmented breast tissue phantoms to estimate dose metrics such as the MGD, this is the first study to examine variation in energy deposition throughout segmented phantoms resulting from mammographic irradiation, considering energy deposition in glandular and adipose tissues. In addition to considering macroscopic breast tissue structure, this is the first study to investigate energy deposition in glandular tissue in the context of mammography on the microscopic level. Sensitivity of results with respect to model parameters is explored. Although previous studies have considered how various model parameters affect dose metrics such as the MGD (see e.g., Sarno et al. ${ }^{159}$ ), this is the first work to consider how such model parameters affect energy deposition on the cellular level.

\subsection{Methods}

\subsubsection{Development of multiscale models of compressed breasts}

Since it is not feasible to model microscopic detail throughout the entire breast, multiscale models of compressed breasts are developed, which combine varying levels of detail on different length scales (figure 7.1). Regions of interest (ROIs) containing mammary epithelial cells and adipocytes are embedded throughout the breast tissue. Nine ROIs are considered: $(0,0,0),(0, \pm 4,0),\left(0,0, \pm z_{\text {shift }}\right)$ and $\left(0, \pm 4, \pm z_{\text {shift }}\right) \mathrm{cm}$ (five are shown in figure 7.1$)$. The location $(0,0,0)$ is defined at $3.75 \mathrm{~cm}$ from the chest wall in the anterior-posterior $(x)$ direction, and centred within the breast in the left-right $(y)$ and inferior-superior $(z)$ directions.

Macroscopic features of the computational models are based on case 3 from AAPM's 
task group (TG) 195. ${ }^{33}$ In this chapter (unlike TG-195), breast tissue is segmented into voxels with $0.5 \mathrm{~mm}$ side length. Most results are presented for simulations involving breast tissue based on phantom 012204 from the database of computational breast phantoms (derived from MRIs of pendant breasts) provided by Zastrow et al. ${ }^{160}$ The glandularity of breast phantom 012204 is closest to what is typical according to Yaffe et $a l .{ }^{161}$ This phantom is trimmed to fit inside the breast tissue region of the compressed breast phantom shown in figure 7.1 (segmented breast tissue is not illustrated, for simplicity); the trimmed phantom has a glandularity of $17 \%$ by mass, with adipose making up the remainder. Since breast phantom 012204 does not fill the entire TG-195 compressed breast volume, the remaining voxels are filled with a homogeneous mixture of glandular and adipose tissues, with $17 \%$ glandularity by mass. The three 'fatty' tissues reported by Zastrow et al. ${ }^{160}$ are reclassified as adipose tissue, the three 'fibroconnective/glandular' tissues are reclassified as glandular tissue, and the 'transitional' tissue is assigned to a mixture containing $50 \%$ glandular and $50 \%$ adipose tissue by mass. Elemental compositions and densities of water, air, skin and PMMA are the same as those used in TG-195. Adipose and glandular tissue compositions and densities are obtained from Woodard and White. ${ }^{84}$

Each ROI contains at least 1000 mammary epithelial cells and $\sim 200$ adipocytes; a scenario involving no adipocytes is also considered (figure 7.2). Mammary epithelial cells are modelled as two concentric spheres, with cytoplasm and nucleus compartments. Adipocytes have a third compartment containing lipid. Cellular compartment sizes are randomly sampled from normal distributions. Cellular compartment sizes for mammary epithelial cells are taken as the average of breast cancer cell data presented by Cai et al.: ${ }^{102}$ mean cell (nucleus) radius is $r_{c e l l, e p i}=8.9 \mu \mathrm{m}\left(r_{n u c, e p i}=5.8 \mu \mathrm{m}\right)$. Adipocyte lipid droplet size is determined from a literature survey of lipid droplet mass per cell for normal, human adults, ${ }^{162-165}$ yielding a mean radius of $r_{\text {lipid }}=51 \mu \mathrm{m}$. The mean adipocyte nuclear radius is taken to be $r_{n u c, a d i}=1.5 \mu \mathrm{m}$, as suggested by Beaulieu et 


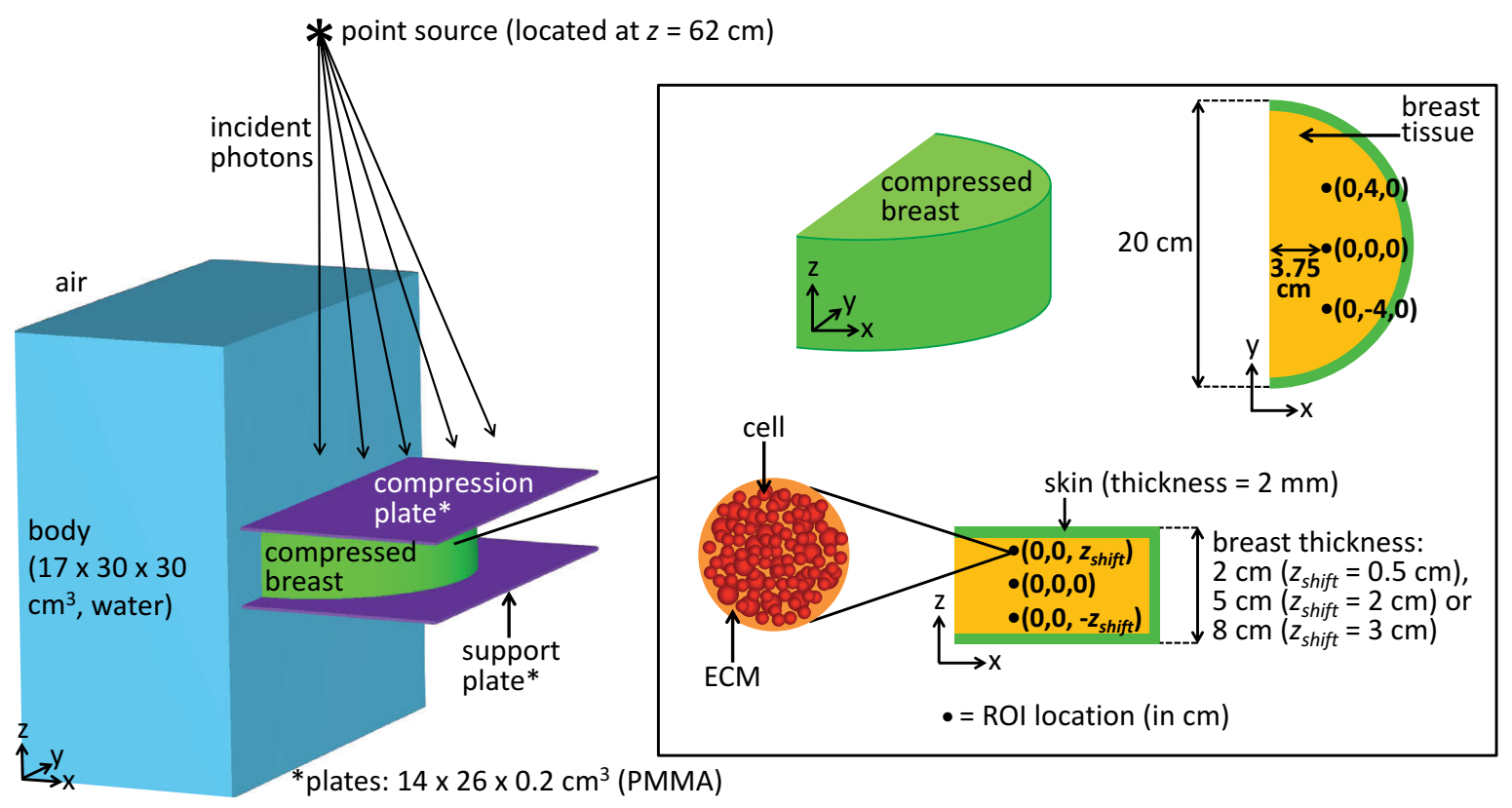

Figure 7.1: Multiscale mammography model with compressed breast (left), and detail (right), showing cross sections through the breast, which is compressed between two PMMA plates. The 'body' is modelled as a rectangular prism of water. A point source (30 kVp Mo/Mo spectrum) is located at $z=62 \mathrm{~cm}$. ROIs (black dots) contain explicitly modelled mammary epithelial cells and adipocytes embedded in an extracellular matrix (ECM). In addition to the five locations shown here, cell populations are also located at $\left(0, \pm 4, \pm z_{\text {shift }}\right) \mathrm{cm}$. 
$a l .{ }^{32}$ Mean adipocyte cell radius is set to $r_{\text {cell }, \text { adi }}=53 \mu \mathrm{m}$ to encompass the lipid and nucleus compartments. The standard deviation on all radii except for $r_{n u c, a d i}$ is $1.0 \mu \mathrm{m}$, which is motivated by uncertainties reported by Cai et al.; ${ }^{102}$ a standard deviation of $0.2 \mu \mathrm{m}$ is assumed for $r_{n u c, a d i}$.

(a) cell configuration \#1

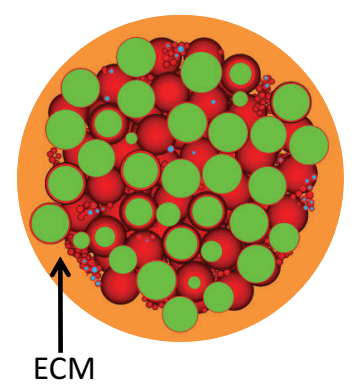

(b) cell configuration \#2

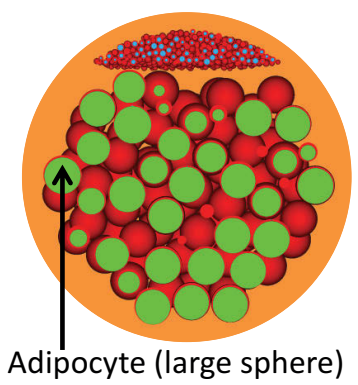

(c) cell configuration \#3

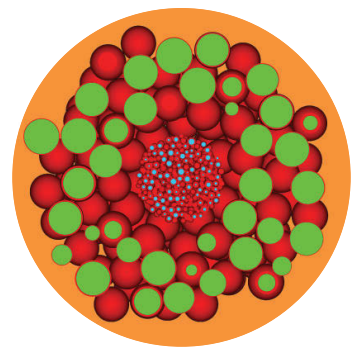

(d) cell configuration \#4

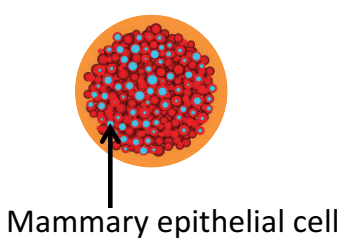

(small sphere)

Figure 7.2: Cross sections of microscopic tissue structure models involving (a) 1291 mammary epithelial cells interspersed among 172 adipocytes, (b) separate mammary epithelial cell and adipocyte clusters consisting of 1567 and 206 cells, respectively, (c) 1351 mammary epithelial cells surrounded by 273 adipocytes, and (d) 1000 mammary epithelial cells (no adipocytes). Cells are embedded in an extracellular matrix (ECM; radius of 126 to $480 \mu \mathrm{m}$ ), which is embedded in the breast tissue at one of the ROI locations indicated in figure 7.1 .

To investigate how cell arrangement method affects energy deposition within mammary epithelial cell nuclei, four cell configurations are considered (figure 7.2). Cells are embedded in an extracellular matrix (ECM). Most results are presented for configuration \#1 (arbitrary choice). Cell positions are obtained from the results of pouring simulations carried out using the LAMMPS Molecular Dynamics Simulator. ${ }^{134}$ The number of adipocytes in cell configurations \#1, \#2 and \#3 is determined by requiring that the total mass of adipocyte lipid droplets divided by the total mass of the ROI is equal to the lipid fraction of glandular bulk tissue $(30.9 \%$ by mass for Woodard and White $\left.^{84}\right)$. Mammary epithelial cells fill in the remaining ROI volume, requiring a minimum cell separation distance of $2.06 \mu \mathrm{m} .{ }^{35}$ For cell configuration \#1 (figure 7.2(a)), the minimum separation distance between mammary epithelial cells and adipocytes is 
increased to $11 \mathrm{\mu m}$ so that the ROI volume and number of epithelial cells is similar to the other cell configurations.

Nucleus and cytoplasm elemental compositions are based on normal mouse mammary epithelial cells ${ }^{92}$ (composition ' $\mathrm{g}$ ' in table 2.1; lipid elemental composition is also given in this table). The ECM is defined such that the average composition and density of the cells plus ECM is approximately equivalent to that of glandular bulk tissue. A different ECM elemental composition and density is calculated for each of the four cell configurations (figure 7.2).

The sensitivity of energy deposition on the microscopic scale with respect to various macro- and microscopic model parameters is explored. Compressed breast thicknesses of 2 and $8 \mathrm{~cm}$ are considered, with $z_{\text {shift }}=0.5$ and $3 \mathrm{~cm}$, respectively, in addition to the $5 \mathrm{~cm}$ thickness mentioned above, with $z_{\text {shift }}=2 \mathrm{~cm}$ (figure 7.1 ). In addition to the segmented breast tissue model described above (based on phantom 012204; $17 \%$ glandularity by mass) we also consider phantoms 012804 (3.8\% glandularity by mass) and 062204 (37\% glandularity by mass) from Zastrow et al. ${ }^{160}$ Homogeneous breast tissue which is a mixture of glandular and adipose tissues is also considered. In addition to the glandular tissue definition given by Woodard and White, ${ }^{84}$ that of Hammerstein et al. ${ }^{166}$ is also considered. Nucleus and cytoplasm elemental compositions based on malignant instead of normal mouse mammary epithelial cells are also considered (composition 'b' in table 2.1). A smaller mammary epithelial cell mean nuclear radius of $2.8 \mathrm{\mu m}$ is also considered, based on data from normal mammary epithelial cells reported by Pienta and Coffey. ${ }^{105}$

\subsubsection{Monte Carlo simulations}

A point source located at $(-3.75,0,62) \mathrm{cm}$ (figure 7.1 ) emits photons according to a $30 \mathrm{kVp}$ mammography spectrum with Molybdenum target and $0.0386 \mathrm{~mm}$-thick Molyb- 
denum filter (Mo/Mo). ${ }^{33}$ Photons are collimated (using the egs_collimated_source from the EGSnrc $\mathrm{C}++$ class library $^{73}$ ) such that they are aimed toward a $14 \times 26 \mathrm{~cm}^{2}$ rectangle in the $x y$-plane, situated $1.5 \mathrm{~cm}$ below the bottom of the breast skin.

The user-code egs_chamber, from the EGSnrc MC software, ${ }^{28,72}$ is used to score specific energy $z$ in mammary epithelial cell nuclei. The mean $(\bar{z})$, standard deviation $\left(\sigma_{z}\right)$, and distribution $\left(f(z, D)\right.$; normalized such that $\left.\int_{0}^{\infty} f(z, D) d z=1\right)$ of the specific energy for mammary epithelial cell nuclei are calculated for each ROI; $\sigma_{z} / \bar{z}$ is the relative standard deviation of the specific energy; $f(z=0)$ is the fraction of nuclei receiving no energy deposition. The glandular voxel dose $\left(D_{g l, R O I}\right)$ is the dose to a sphere of glandular tissue occupying the space of the cell population and ECM; a distinct value of $D_{g l, R O I}$ is calculated for each ROI. Because $D_{g l, R O I}$ is scored in a region whose elemental composition differs from that of mammary epithelial cell nuclei, $\bar{z}$ is generally not equal to $D_{g l, R O I}$. MC simulations are split up across hundreds of jobs on different computers; results for different dose levels are obtained by adding up results from separate jobs, resulting in dose resolution of $0.03 \mathrm{mGy}$ or better.

The EGSnrc user-code egs_brachy ${ }^{81}$ is used to compute macroscopic dose distributions within segmented breast tissue models based on phantoms 012204, 012804 and 062204 from Zastrow et al. ${ }^{160}$ No electrons are transported, and a tracklength estimator is used to score dose-to-medium within each of the $(0.5 \mathrm{~mm})^{3}$ voxels of the segmented phantom, as described in chapter 2. To compute glandular tissue doses, egs_brachy's 'muen for medium' option is used to score dose to glandular tissue while transport is carried out in voxels containing mixtures of glandular and adipose tissues. MGD is estimated by averaging dose to glandular tissue across the entire phantom, with voxel contributions weighted according to glandularity. Statistical uncertainties on voxel doses and on the MGD are $<0.005 \mathrm{mGy}$ and $1 \times 10^{-6} \mathrm{mGy}$, respectively (for all phantoms considered herein). 


\subsection{Results}

\subsubsection{Macroscopic dose distributions in segmented breast tis- sue phantoms}

Dose distributions at three locations along the $z$-axis within the compressed breast model based on phantom 012204 from Zastrow et al. ${ }^{160}$ are shown in figure 7.3: (a) and (b) correspond to a location near the top surface of the breast, (c) and (d) correspond to the centre of the breast, and (e) and (f) correspond to a location 'deeper' in the breast tissue, further away from the radiation source. The maximum dose represented by the colour plot decreases from $\sim 12$ mGy to $\sim 0.70$ mGy as $z$ decreases from $2 \mathrm{~cm}$ to $-2 \mathrm{~cm}$, due to attenuation of the incident photons. Dose distributions at the centre of the breast for two additional models are presented in figure 7.4, demonstrating considerable variation in breast tissue structure, and in the corresponding dose distribution. In all cases, $\mathrm{MGD}=4 \mathrm{mGy}$. Doses to voxels containing glandular tissue vary between 0.3 and 15 mGy for phantom 012204, between 0.5 and 17 mGy for phantom 012804 and between 0.2 and 16 mGy for phantom 062204, with glandularities of 17\%, 3.8\% and $37 \%$ by mass, respectively.

For the energy range of interest in mammography, the mass energy absorption coefficients $\left(\mu_{e n} / \rho\right)$ of adipose tissue are lower than those of glandular tissue. ${ }^{167}$ Therefore, it is generally true that relatively low (high) doses are observed in figures 7.3 and 7.4 in voxels containing adipose (glandular) tissue. The tissue structure located upstream plays an important role in determining downstream dose. Voxel doses are generally lower near the core of the breast where glandular tissue tends to be concentrated, resulting in attenuation of the incident fluence - see low dose regions in figure 7.3(f) due to upstream glandular tissue (figure 7.3(a)). Such 'shadows' are also apparent in figure 7.4(b); these low dose regions are unrelated to the tissue structure of that particular layer of voxels (figure 7.4(a)). Phantom 012804 (3.8\% glandularity 
(a) tissue structure, $z=2 \mathrm{~cm}$

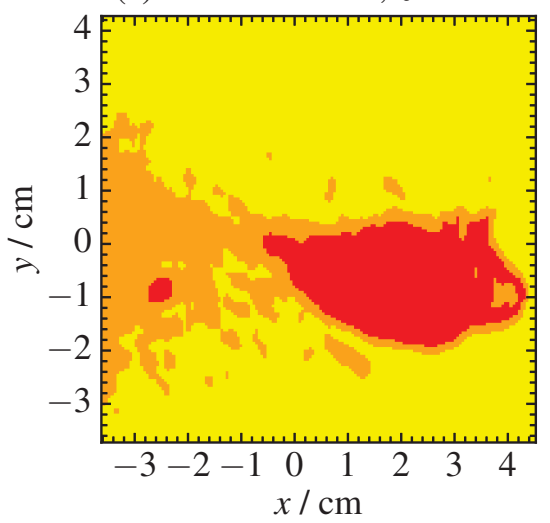

(c) tissue structure, $z=0 \mathrm{~cm}$

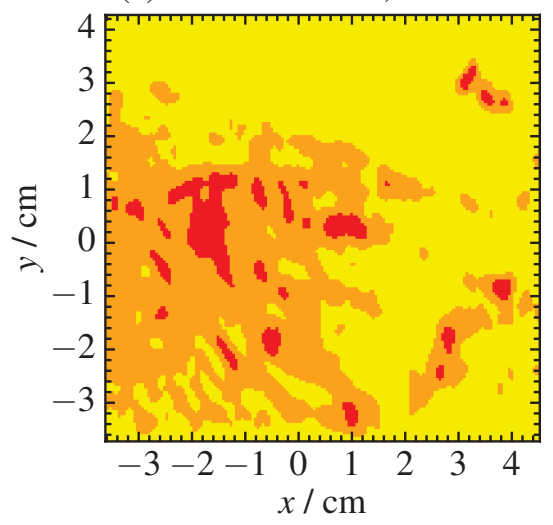

(e) tissue structure, $z=-2 \mathrm{~cm}$

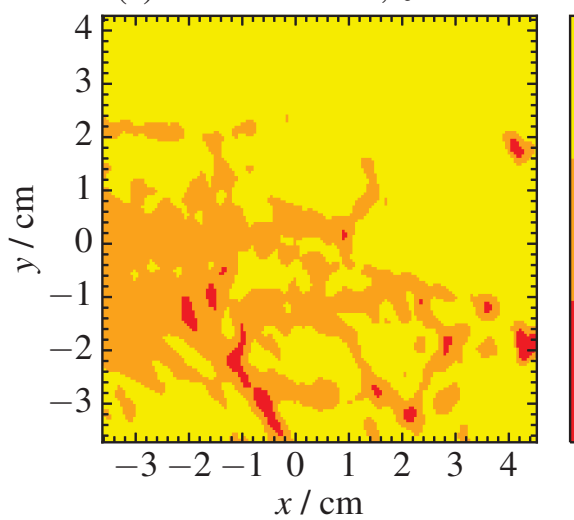

(b) dose distribution, $z=2 \mathrm{~cm}$

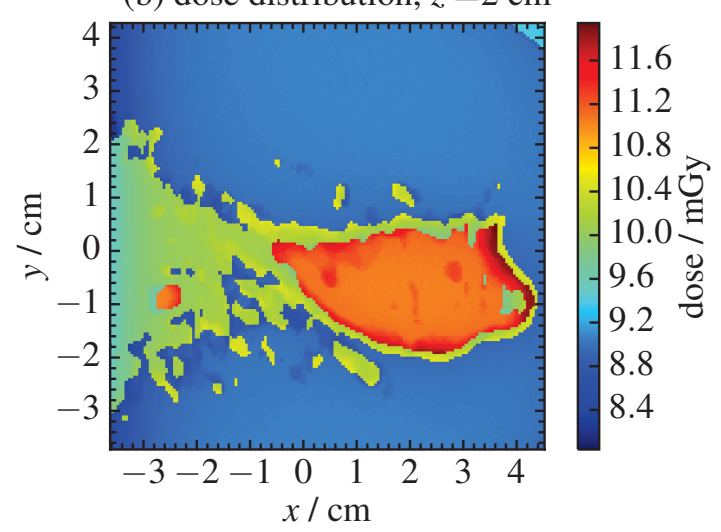

(d) dose distribution, $z=0 \mathrm{~cm}$

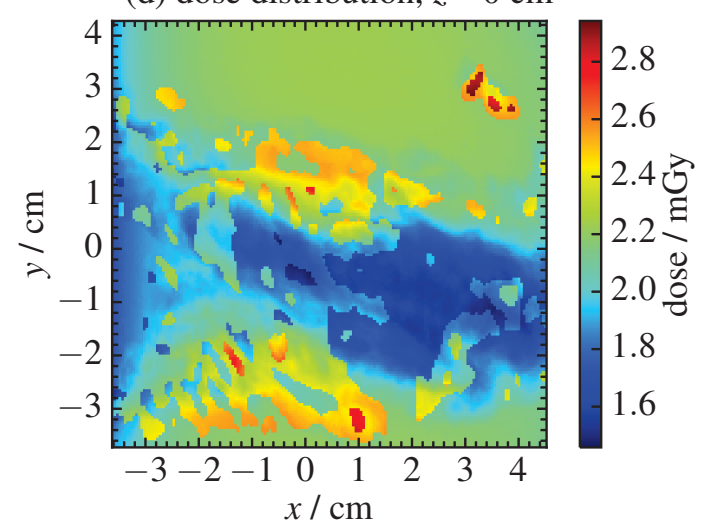

(f) dose distribution, $z=-2 \mathrm{~cm}$

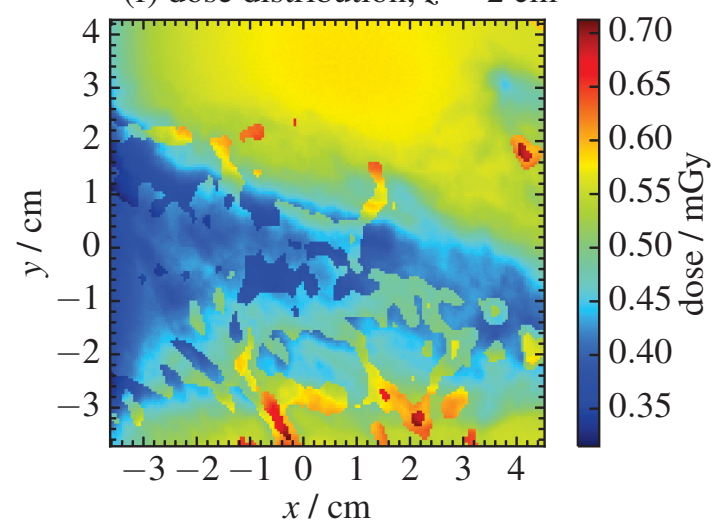

Figure 7.3: Detail of segmented breast tissue structure, and corresponding dose distributions for $\mathrm{MGD}=4 \mathrm{mGy}$, for a breast phantom with $17 \%$ glandularity by mass, $5 \mathrm{~cm}$ compressed thickness, based on phantom 012204 ('scattered fibroglandular tissue'), using the glandular tissue composition of Woodard and White ${ }^{84} z=(\mathrm{a}, \mathrm{b}) 2 \mathrm{~cm},(\mathrm{c}, \mathrm{d}) 0 \mathrm{~cm}$, and $(\mathrm{e}, \mathrm{f})-2 \mathrm{~cm}$. 
(a) tissue structure, phantom ID 012804

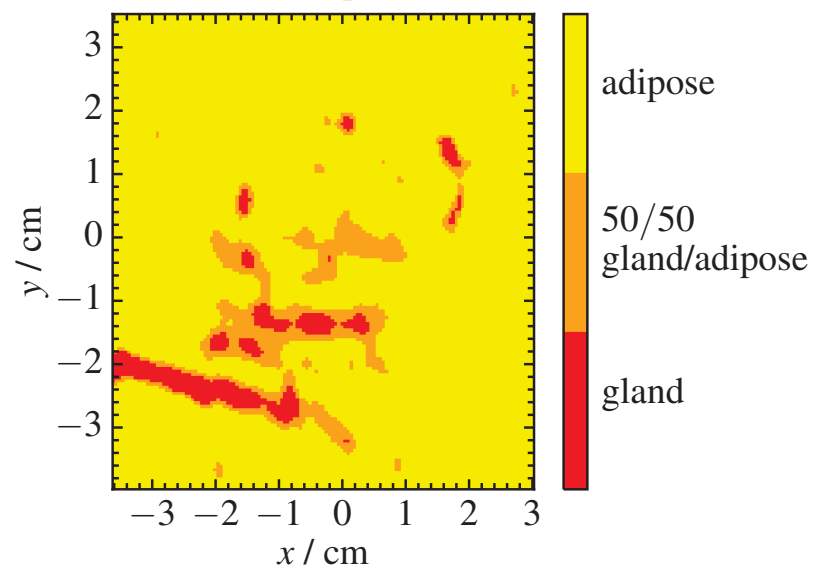

(c) tissue structure, phantom ID 062204

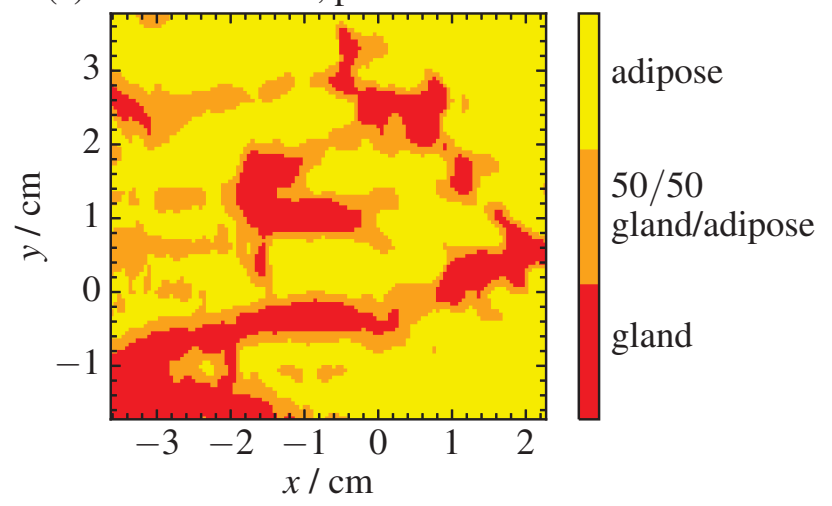

(b) dose distribution, phantom ID 012804

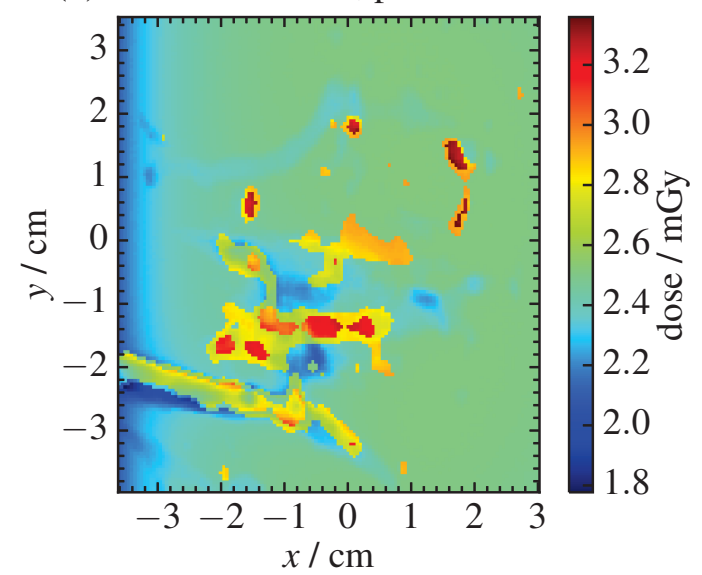

(d) dose distribution, phantom ID 062204

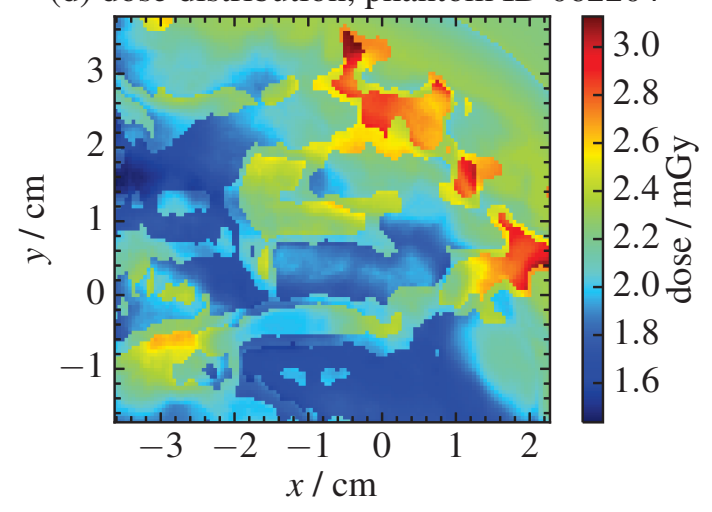

Figure 7.4: As in figure 7.3, but for (a,b) a 'mostly fatty' breast (based on phantom 012804, 3.8\% glandularity by mass), and (c,d) a 'heterogeneously dense' breast (based on phantom 062204, 37\% glandularity by mass); $z=0 \mathrm{~cm} ; \mathrm{MGD}=4 \mathrm{mGy}$. 
by mass; figures $7.4(\mathrm{a}, \mathrm{b})$ ) ends up with relatively high dose throughout the glandular tissue, since there is little upstream glandular tissue to shield that which is deeper in the phantom. Phantom 062204 (37\% glandularity by mass; figures 7.4(c,d)) demonstrates considerable variation in dose throughout the glandular tissue. Lack of scatter from air contributes to lower dose in the breast tissue near the outer edges of the phantom (not shown). Dose is also relatively low near the chest wall (i.e., $x=-3.75$ ) because this is the edge of the radiation field, hence there is little radiation scattered into the breast from the water phantom representing the body (figure 7.1).

\subsubsection{Specific energy distributions within cell populations}

Due to attenuation, specific energy distributions for mammary epithelial cell nuclei are strongly dependent on ROI $z$-coordinate (figure $7.5(\mathrm{a})) . f(z, D)$ also vary somewhat with changes in the $y$-coordinate; this dependence is likely due to differences in upstream tissue structure with e.g., less glandular tissue upstream resulting in less attenuation and an $f(z, D)$ that is shifted toward higher specific energies. $f(z, D)$ for different ROIs, but for the same $D_{g l, R O I}$ are in good agreement (not shown). For smaller $D_{g l, R O I}$, $f(z, D)$ is more skewed (less normal), has a larger fraction of targets receiving no energy deposition $(f(z=0))$, and has a larger relative standard deviation $\left(\sigma_{z} / \bar{z}\right)$ - see figure 7.5(b). Figure 7.5(c) illustrates the variation in $\bar{z}$ and $D_{g l, R O I}$ that occurs throughout the breast due to differences in attenuation and scatter, demonstrating that $D_{g l, R O I}$ varies between 1 and $24 \mathrm{mGy}$, with the ROIs located at $z=2 \mathrm{~cm}$ receiving the most energy deposition because they are closest to the radiation source. Because the elemental compositions of mammary epithelial cell nuclei and their surroundings are different

from that of glandular tissue, $\bar{z}$ exceeds $D_{g l, R O I}$ by $\sim 25 \%$. The MGD agrees most closely with $D_{g l, R O I}$ for the ROIs at $z=0 \mathrm{~cm}$ (discrepancy $\simeq 50 \%$ ).

Figure 7.6 demonstrates that $\sigma_{z} / \bar{z}$ decreases as a function of $D_{g l, R O I}$, in agreement with figure $7.5(\mathrm{~b})$. As dose increases, the average number of energy deposition events 

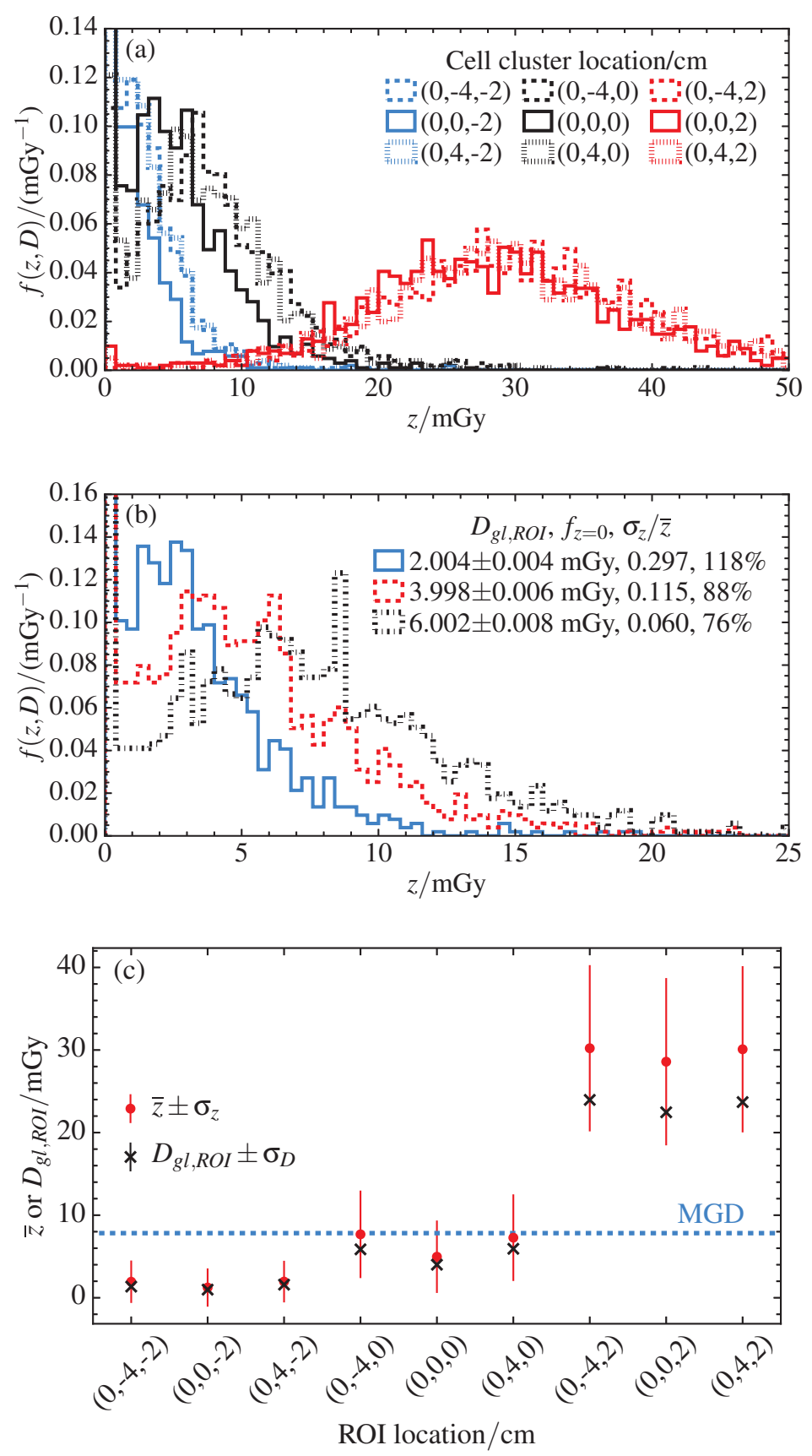

Figure 7.5: Specific energy distributions for mammary epithelial cell nuclei for (a) different ROIs for a given number of histories and (b) different values of $D_{g l, R O I}$ for the ROI at $(0,0,0)$; the leftmost bin is truncated in some cases. In (a) and (c), $D_{g l, R O I}=3.998 \pm$ $0.006 \mathrm{mGy}$ for the ROI at $(0,0,0)$. Values of $\bar{z}, D_{g l, R O I}$ and corresponding MGD are presented in (c) as a function of ROI location; error bars on $\bar{z}$ are $\sigma_{z}$; error bars on $D_{g l, R O I}$ are MC statistical uncertainties (too small to see). Breast thickness: $5 \mathrm{~cm}$, segmented breast tissue based on phantom 012204 (glandularity: 17\% by mass), glandular tissue: Woodard and White, ${ }^{84}$ cellular media: normal mouse epithelial cells, cell configuration \#1 (figure $7.2(\mathrm{a})$ ), and $r_{n u c, e p i}=5.8 \mu \mathrm{m}$. 
per target increases so that there is less variation in specific energy resulting from the stochastic nature of radiation. For a typical $D_{g l, R O I}$ of $4 \mathrm{mGy}, \sigma_{z} / \bar{z} \simeq 85 \%$. As $D_{g l, R O I}$ increases from 0.5 to $6.5 \mathrm{mGy}, \sigma_{z} / \bar{z}$ decreases from $\sim 250 \%$ to $\sim 69 \%$. The slope of $\sigma_{z} / \bar{z}$ as a function of $D_{g l, R O I}$ is larger for lower doses. Values of $\sigma_{z} / \bar{z}$ for different cell configurations are in good agreement for $D_{g l, R O I}>3 \mathrm{mGy}$; results for different ROIs are also in good agreement for $D_{g l, R O I}>3 \mathrm{mGy}$ (not shown). The curves shown in figure 7.6 exhibit discontinuities (i.e., the curves appear jagged), especially for $D_{g l, R O I}<3 \mathrm{mGy}$; low doses correspond to fewer interactions occurring within the ROI, resulting in an increase in statistical fluctuations; some nuclei may receive no energy deposition at all (figure 7.5(b)). Furthermore, not every cell/nucleus in the population is the same size; therefore, if only a small subset of cells contributes to the calculation of $\bar{z}$ or $\sigma_{z}$, then the average size of cells/nuclei in this subset may differ from that of the full population.

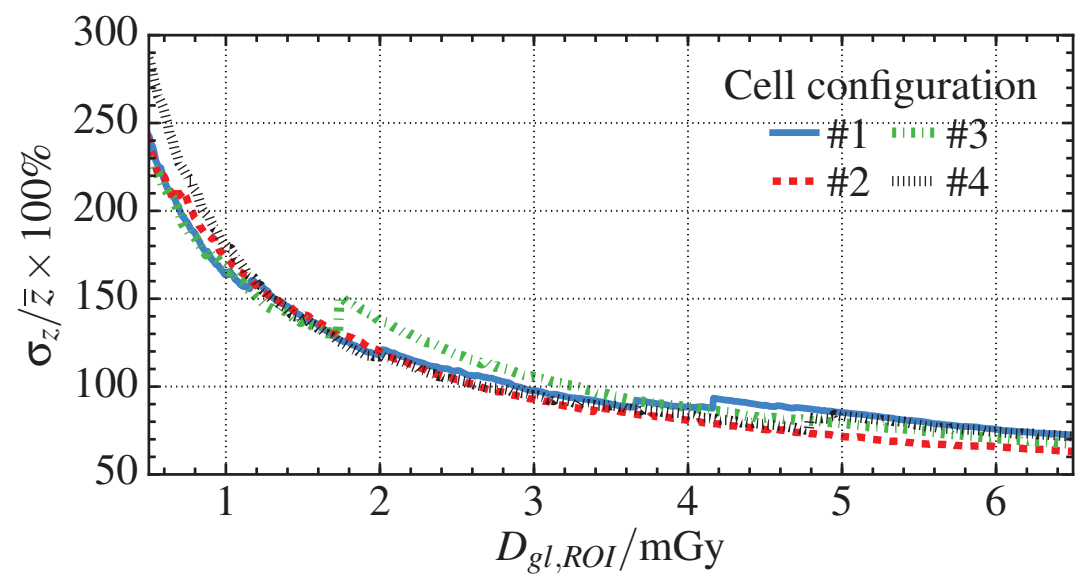

Figure 7.6: Relative standard deviation of the specific energy for mammary epithelial cell nuclei for the ROI at $(0,0,0)$ plotted as a function of the corresponding glandular voxel dose, for different cell configurations (figure 7.2). Breast thickness: $5 \mathrm{~cm}$, segmented breast tissue based on phantom 012204 from Zastrow et al. ${ }^{160}$ (glandularity: 17\% by mass), glandular tissue: Woodard and White, ${ }^{84}$ cellular media: normal mouse epithelial cells, and $r_{n u c, e p i}=5.8 \mu \mathrm{m}$. Data points are joined with a line to guide the eye. Error bars are not shown since they are comparable to or smaller than the line width. 


\subsubsection{Sensitivity analysis for specific energy distributions}

Figure 7.7 demonstrates that $f(z, D)$ for mammary epithelial cell nuclei for a given $D_{g l, R O I}$ is approximately independent of many macro- and microscopic model parameters including compressed breast thickness, glandularity, glandular tissue composition, breast tissue type (i.e., homogeneous or segmented) and cell configuration. However, results are sensitive to cell model details. There is considerable variation in $f(z, D)$ for the two values of $r_{n u c, e p i}$ considered herein (figure $7.7(\mathrm{~g})$ ); $\sigma_{z} / \bar{z}=227 \%$ and $88 \%$ for $r_{n u c, e p i}=2.8$ and $5.8 \mu \mathrm{m}$, respectively. For the same $D_{g l, R O I}$, more interactions will occur within a larger target, resulting in less stochastic fluctuations; therefore, $\sigma_{z} / \bar{z}$ is smaller for the larger target size. Although $f(z, D)$ for different cellular elemental compositions (figure $7.7(\mathrm{f})$ ) appear similar, $\bar{z}$ exceeds $D_{g l, R O I}$ by $\sim 25 \%$ and $\sim 33 \%$ for normal and malignant cellular elemental compositions, respectively. Considering figure 7.7(d) for other ROIs, $f(z, D)$ remains insensitive to whether the breast tissue is homogeneous or segmented (results not shown). In principle, certain breast tissue structures could affect the fluence reaching the ROI, which could lead to differences in $f(z, D)$; however, this potential effect is not observed for the breast tissue structure considered herein. Furthermore, $f(z, D)$ for a given $D_{g l, R O I}$ is approximately insensitive to cell configuration (figure 7.7(e)), even for configuration \#4 (figure 7.2(d)), which is considered an unrealistic scenario since no adipocytes are modelled, and the lipid content is contained within the ECM.

\subsection{Discussion}

This chapter presents a novel investigation of breast dosimetry for mammography, considering energy deposition within glandular tissues on macro- and microscopic length scales using multiscale MC simulations. We compute macroscopic dose distributions in segmented breast tissue phantoms, considering energy deposition within voxels contain- 

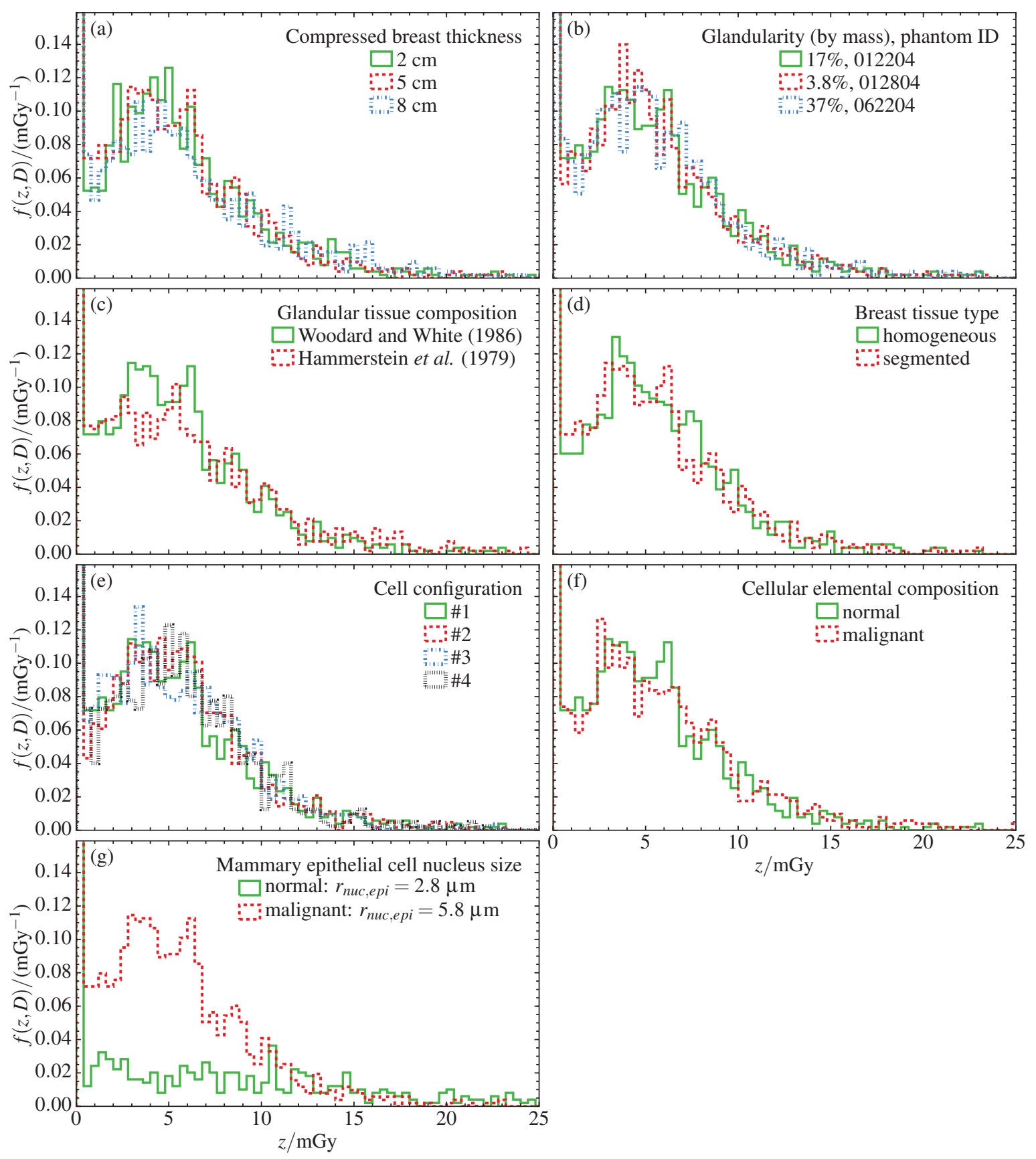

Figure 7.7: Specific energy distributions for mammary epithelial cell nuclei demonstrating sensitivity to (a) compressed breast thickness, (b) glandularity (i.e., breast tissue structure), (c) glandular tissue composition, (d) breast tissue type, (e) cell configuration (figure 7.2), (f) cellular elemental composition, and (g) nucleus size. One parameter is changed at a time relative to the following: breast thickness: $5 \mathrm{~cm}$, segmented breast tissue based on phantom 012204 (glandularity: $17 \%$ by mass), glandular tissue: Woodard and White, ${ }^{84}$ cellular media: normal mouse epithelial cells, cell configuration \#1 (figure 7.2(a)), and $r_{n u c, e p i}=5.8 \mu \mathrm{m}$, considering the ROI at $(0,0,0)$, with $D_{g l, R O I}=4 \pm 0.03 \mathrm{mGy}$. 
ing glandular and/or adipose tissues — such dose distributions have not been presented previously in the literature. This study is also the first to consider energy deposition in the underlying microscopic tissue structure resulting from a typical mammography exam.

Macroscopic dose distributions presented in figures 7.3 and 7.4 demonstrate considerable variation in dose throughout the breast; doses to voxels containing glandular tissue vary between $\sim 0.1$ and $\sim 4$ times the MGD. The general trend observed in e.g., figure 7.3 of decreasing dose with increasing depth in breast tissue is in agreement with the glandular tissue dose distribution shown in figure $7(\mathrm{a})$ of Sechopoulos et al. ${ }^{154}$ In addition to consideration of macroscopic dose distributions, our multiscale breast models also allow for investigation of energy deposition on microscopic length scales: the considerable magnitude of $\sigma_{z} / \bar{z}$ for typical mammographic dose levels is demonstrated in figure 5.7 (e.g., $\sigma_{z} / \bar{z}=85 \%$ when $\left.D_{g l, R O I}=4 \mathrm{mGy}\right)$, and is reflected by the errors bars on values of $\bar{z}$ presented in figure $7.5(\mathrm{c})$. The considerable width of the specific energy distribution can also be observed in figure 7.5(a). For the scenario considered in figure $7.5(\mathrm{c})$, while $D_{g l, R O I} \simeq 4 \mathrm{mGy}$ at $(0,0,0), D_{g l, R O I} \simeq 24 \mathrm{mGy}$ at $(0, \pm 4,2)$ with $\bar{z}$ systematically exceeding $D_{g l, R O I}$ by $27 \%$ (yielding $\bar{z} \simeq 30 \mathrm{mGy}$ ), and $1 \sigma$ standard deviation of the specific energy resulting in specific energies of up to $\sim 40 \mathrm{mGy}$, or 10 times the $D_{g l, R O I}$ at $(0,0,0)$. Therefore, for a typical mammography procedure, some mammary epithelial cell nuclei receive energy deposition considerably larger than expected based on knowledge of $\bar{z}, D_{g l, R O I}$ or MGD. This result may have implications for mammography radiation protection given that cancer induction risk varies (increasing in general) with dose level. ${ }^{11}$

The MGD and $D_{g l, R O I}$ agree within $\sim 50 \%$ for the ROIs located at $z=0 \mathrm{~cm}$, with much larger discrepancies for the ROIs located at different depths. Reporting the MGD fails to provide any description of the two types of variation described in the previous paragraph (i.e., variation throughout a population of cells due to the stochastic nature 
of radiation, and macroscopic variations in dose throughout the breast due to differences in attenuation and scatter). Dance and Sechopoulos ${ }^{149}$ point out that the MGD should not be interpreted as an accurate estimate of breast dose; instead, the MGD is a useful and convenient metric for examining relative trends in breast dose. More detailed computational models for breast dosimetry could be used to develop better informed mammography risk assessment, based on more than a single dose estimate such as the MGD. However, risk estimation is beyond the scope of this chapter.

Yaffe and Mainprize ${ }^{168}$ conclude that the risk for radiation-induced breast cancer arising from a typical mammographic screening program is 'small' relative to the number of lives saved by cancer diagnosis and subsequent treatment. Nonetheless, recent research has begun to question the accuracy of traditional breast cancer risk assessment protocols for mammography, which are often based on data from atomic bomb survivors who were exposed to radiation at a higher energy than what is used in mammography. The relatively low energy x-rays used in mammography are potentially twice as damaging per unit dose due to their increased linear energy transfer (LET). ${ }^{169}$ However, Mills et al. ${ }^{170}$ point out that photoelectric events are the dominant interaction type for typical mammography photon energies so that the spectrum of electrons set into motion by mammography x-rays is similar to that which is generated by $200 \mathrm{keV}$ photons, which are likely to undergo Compton scattering. Furthermore, the microdosimetric analysis carried out by Verhaegen and Reniers ${ }^{171}$ indicates that the dose-mean lineal energy of mammography x-rays varies with depth, and depends on choice of target/filter combination. Finally, risk assessment for mammography often involves extrapolation of the dose-response curve to low doses, where the linear no-threshold model is assumed, but not verified. ${ }^{11}$

Figure 7.7 illustrates that $f(z, D)$ for mammary epithelial cell nuclei are approximately insensitive to various model parameters, provided that $D_{g l, R O I}$ is held constant. Therefore, if the glandular voxel dose is known, then the corresponding $f(z, D)$ could 
be obtained from a database of specific energy distributions corresponding to different dose levels. However, $f(z, D)$ are weakly sensitive to cellular elemental composition (figure $7.7(\mathrm{f})$ ), and strongly sensitive to the values of $r_{n u c, \text { epi }}$ considered herein (figure $7.7(\mathrm{~g}))$. This result emphasizes the need for accurate cell models, and highlights dosimetric differences between healthy and malignant tissues. Cellular elemental compositions considered herein are based on mouse mammary epithelial cells (see section 7.2.1); results may vary if human cell media were available.

The structure and composition of the breast varies from woman to woman; figures 7.3 and 7.4 demonstrate variation in breast tissue structure (and corresponding variation in the dose distribution) for three example cases, with glandularities ranging from $3.8 \%$ to $37 \%$ by mass. In fact, glandular structure varies with age, menstrual cycle stage, lactation status and depends on whether or not the woman has given birth. ${ }^{68,172} \mathrm{Al}$ though the glandular tissue definition of Hammerstein et al. ${ }^{166}$ is frequently used in the mammography dosimetry literature, ${ }^{149}$ Woodard and White ${ }^{84}$ is primarily used herein because it is a more recent publication which cites Hammerstein et al. ${ }^{166}$ Woodard and White $^{84}$ use seven specimens taken from post-menopausal women, while Hammerstein et al. ${ }^{166}$ use only five samples. Regardless, figure $7.7(\mathrm{c})$ demonstrates that $f(z, D)$ for a given $D_{g l, R O I}$ is insensitive to glandular tissue composition. Tomal et al. ${ }^{173}$ report that neoplastic breast tissues have linear attenuation coefficients that are higher than those of normal glandular tissue, potentially due to an increased concentration of higher atomic number elements. Future work may investigate the dosimetric consequences of this change in elemental composition.

There is a lack of segmented, compressed breast computational phantoms available in the literature. In this chapter, we use data from Zastrow et al., ${ }^{160}$ who provide data for (uncompressed) pendant breasts. Future work may investigate more sophisticated compressed breast phantoms: for example, Sechopoulos et al. ${ }^{154}$ use a compression algorithm based on a linear spring model ${ }^{157}$ to simulate compression of DBCT images 
of pendant breasts. Another strategy involves developing generic segmented breast tissue models that are representative of some subset of the population. ${ }^{155}$ Nonetheless, the segmented breast tissue models used herein are arguably more realistic than the homogeneous models used in traditional mammography breast dosimetry studies, and are likely adequate for exploring general trends in macroscopic dose distributions (figures 7.3 and 7.4), and for investigating sensitivity of $f(z, D)$ to homogeneous versus segmented breast tissue (figure $7.7(\mathrm{~d})$ ).

In this chapter, the body is modelled as a rectangular water phantom; the contralateral breast is not modelled. Wang et al. ${ }^{156}$ have demonstrated that inclusion of a whole-body voxel phantom affects the glandular dose coefficient (ratio of MGD and incident air kerma) by at most 3.1\% compared to an isolated breast model. Therefore, the impact of using a water phantom for the body (as in this chapter) instead of a whole-body voxel phantom is expected to be $<3.1 \%$. Future research may investigate how mammary epithelial cell nuclear specific energy distributions are affected by the presence of calcifications, which have been shown to result in considerable perturbation of the dose distribution in the context of iodine-125 prostate brachytherapy. ${ }^{174}$ We assume a skin thickness of $2 \mathrm{~mm}$, and support/compression plate thicknesses of $2 \mathrm{~mm}$. Sarno et al. ${ }^{159}$ report a polyenergetic normalized glandular dose coefficient $\left(\mathrm{DgN}_{p}\right)$ that is $19 \%$ smaller when skin thickness increases from $1.45 \mathrm{~mm}$ to $4 \mathrm{~mm}$ for a $30 \mathrm{kVp}$ Mo/Mo spectrum; removing the 2 mm-thick PMMA compression plate causes $\mathrm{DgN}_{p}$ to increase by $<1.5 \%{ }^{159}$ Therefore, care should be taken to model the skin and plate thicknesses accurately. Our simulations involve a point source located at $z=62 \mathrm{~cm}$, corresponding to a cranial-caudal view; however, a typical mammographic exam also includes a mediolateral oblique view. We only consider a $30 \mathrm{kVp} \mathrm{Mo/Mo} \mathrm{spectrum;} \mathrm{however,} \mathrm{various}$ target/filtration materials and tube voltages are routinely used in mammography. ${ }^{149}$ 


\subsection{Conclusions}

In this chapter, novel, multiscale breast models are used to investigate energy deposition in glandular tissues in the context of mammography. We consider mammography breast dosimetry beyond a single macroscopic dose metric such as the MGD, which has been the primary focus of previous studies. Considering macroscopic length scales, there is considerable variation in dose throughout the breast (glandular voxel doses vary between $\sim 0.1$ and $\sim 4$ times the MGD) due to differences in attenuation and scatter, as well as variation with glandularity and tissue structure, which varies from woman to woman. In addition to this macroscopic dose variation, our multiscale MC simulations demonstrate that there is considerable variation in specific energy throughout a population of mammary epithelial cell nuclei: e.g., $\sigma_{z} / \bar{z}=85 \%$, for a typical $D_{g l, R O I}$ of $4 \mathrm{mGy}$. Furthermore, the mean specific energy for mammary epithelial cell nuclei exceeds dose to the corresponding glandular tissue voxel by $\sim 30 \%$ for a typical mammographic irradiation. Therefore, $D_{g l, R O I}=4 \mathrm{mGy}$ corresponds to $\bar{z} \simeq 5 \mathrm{mGy}$, with a $1 \sigma$ standard deviation resulting in specific energies of up to $\sim 9 \mathrm{mGy}$. If $D_{g l, R O I}=4 \mathrm{mGy}$ corresponds to the ROI at $(0,0,0)$, then a ROI that is $2 \mathrm{~cm}$ closer to the radiation source will have specific energies of up to $\sim 40$ mGy. MGD, while commonly used in mammography breast dosimetry studies, does not account for variation in energy deposition on macroor microscopic length scales. We demonstrate that $f(z, D)$ for mammary epithelial cell nuclei are sensitive to cellular elemental composition and nuclear radius, while being approximately insensitive to compressed breast thickness, glandularity, glandular tissue composition, breast tissue type (i.e., homogeneous or segmented), and cell configuration, provided that $D_{g l, R O I}$ is constant. Results highlight the importance of considering the stochastic nature of radiation when scoring dose in subcellular targets, and may be relevant for mammography radiation protection, given the radiosensitivity and risk of carcinogenesis associated with mammary epithelial cell nuclei. 


\section{Chapter 8}

\section{Conclusion}

This chapter summarizes the research contributions provided by this thesis. Additionally, opportunities for future research beyond the work presented herein are outlined. 


\subsection{Summary}

The results presented in this thesis advance our understanding of energy deposition on cellular length scales for radiotherapy and diagnostic radiology contexts. Evaluation of energy deposition resulting from medical uses of ionizing radiation typically involves consideration of doses on macroscopic length scales, considering $\sim$ mm-sized voxels or larger volumes of interest (e.g., dose-to-medium/water considered in chapters 3 and 4, the macroscopic dose considered in chapters 5 and 6, the MGD considered in the context of mammography in chapter 7). In contrast, this thesis has investigated energy deposition across multiple length scales, working to connect traditional macroscopic dose descriptors with energy deposition on radiobiologically-relevant cellular length scales, considering nuclear and cytoplasm targets.

Chapter 3 investigated kilovoltage photon irradiation of multicellular models consisting of 13-cell clusters, as well as single cells and single nuclear cavities. A range of cell and nucleus sizes, and various cellular and bulk tissue elemental compositions were considered. Results of this chapter demonstrate that neither water nor bulk tissue are reliable substitutes for subcellular targets for incident photon energies $<50 \mathrm{keV}$. This result is relevant for the debate on dose specification medium for MBDCAs; if cellular targets are considered the relevant radiobiological targets, then this work does not provide particular support for either macroscopic dose descriptor. The largest differences between dose descriptors were seen for the lowest incident photon energies; differences were less than $3 \%$ for energies $\geq 90 \mathrm{keV}$. The sensitivity of results presented in chapter 3 with respect to the parameters of the microscopic tissue structure model, and the importance of the nucleus and cytoplasm as targets for radiation-induced cell death emphasize the importance of accurate models for cellular dosimetry studies. 
In chapter 4, relationships between macroscopic (bulk tissue) and microscopic (cellular) dose descriptors were investigated using cavity theory and MC simulations, considering kilovoltage photon energies. This chapter includes a survey of small, large, and intermediate cavity theory approaches from the literature. Considering $\mu \mathrm{m}$-sized water cavities in bulk tissue phantoms, different cavity theory approaches were evaluated via comparison of $D_{w, m} / D_{m, m}$ with MC results; the best overall agreement was achieved with the 'Burlin-Janssens' ICT approach. Values of $D_{n u c} / D_{m, m}$ computed with this ICT approach were compared with those from MC simulations involving multicellular soft tissue models, considering a representative range of cell/nucleus sizes and elemental compositions. Chapter 4 results suggest that cavity theory may be useful for linking doses from MBDCAs with energy deposition in cellular targets.

The multicellular models used in chapters 3 and 4 consist of 13 uniformly-sized cells in a hexagonal lattice. Chapters 5 and 7 expand on this model by investigating the effect of having a distribution of cell sizes and cell types, with cells arranged randomly, considering populations of $>1000$ cells. Furthermore, chapters 3 and 4 focus on absorbed dose scored in the central cell of a 13-cell cluster, without considering the stochastic nature of radiation transport and energy deposition, which can potentially result in considerable variation in energy deposition throughout a population of cells, especially for low doses and small target sizes. ${ }^{16}$ The results presented in chapters 3 and 4 can be interpreted as pertaining to the mean specific energy, averaged across a population of cells. Detailed investigations of these stochastic variations are presented in chapters 5, 6 and 7.

In chapter 5 , multiscale models of healthy and cancerous human soft tissues consisting of populations of $>1500$ cells were developed, and were used to investigate energy deposition in subcellular targets, quantify the microdosimetric spread, and determine how these results depend on model details for a range of incident photon energies and dose levels. MC simulation results demonstrated that for $\sim \mathrm{mGy}$ doses, there is consid- 
erable microdosimetric spread throughout a population of cells. The results presented in chapter 5 highlight the importance of microdosimetric considerations at low doses. The microdosimetric spread increases with decreasing dose, target size, and incident photon energy. At low energies, macroscopic (i.e., bulk tissue) dose descriptors differ considerably from mean specific energies for subcellular targets, in agreement with results presented in chapter 3 , namely that $D_{n u c} \neq D_{m, m}$ in general. Chapter 5 represents an extension of the work presented in chapter 3, and further emphasizes the importance of accurate cell models.

In chapter $6, \mathrm{MC}$ simulations were used to investigate variation in energy deposition across populations of microscopic targets relevant for Raman spectroscopy studies of cellular radiation response. Simulation geometries involved populations of 1600 cells with cytoplasm and nucleus compartments. Smaller sampling volumes (representing the Raman spectroscopy laser spot size) were located within each nucleus. To investigate variation in the microdosimetric spread as a function of dose and target size, simple cubic voxel geometries were also considered. The work presented in chapter 6 demonstrates that the stochastic nature of radiation is an important consideration for cellular radiation response studies using Raman spectroscopy, especially for low doses. The results of this work may motivate changes to the way that Raman spectroscopy studies are carried out. In addition, the results of chapter 6 motivate future research comparing experimental Raman spectroscopy measurements with computational results, which would potentially lead to a better understanding of the observed variation in radiation response across a population of biological targets.

Chapter 7 concerns the development of multiscale MC simulation models of compressed breasts, which were used to investigate energy deposition in glandular tissues on macro- and microscopic length scales in the context of mammography. Microscopic tissue models consisting of at least 1000 mammary epithelial cells and $\sim 200$ adipocytes were embedded at nine ROIs throughout the breast tissue. Glandular voxel doses un- 
derestimated mammary epithelial cell nuclei mean specific energies, with considerable variations in specific energy throughout corresponding cell populations, in addition to dose variations throughout the breast. Furthermore, a model parameter sensitivity analysis showed that energy deposition within mammary epithelial cell nuclei is sensitive to cell model details including cellular elemental compositions and nucleus size, underlining the importance of realistic cellular models. However, for a given glandular voxel dose, specific energy distributions for mammary epithelial cell nuclei were approximately insensitive to cell configuration (i.e., the arrangement of epithelial cells and adipocytes), compressed breast thickness, glandularity, glandular tissue composition, and whether the breast tissue was modelled as a homogeneous mixture of glandular and adipose tissue or as a segmented model. As in chapters 5 and 6, chapter 7 results highlight the importance of microdosimetric considerations when scoring dose in subcellular targets. Results may be relevant for radiation-induced cancer risk evaluation in mammography, which has traditionally focused on a single metric such as the MGD, which ignores variation in energy deposition on both macro- and microscopic length scales.

\subsection{Outlook and ideas for future work}

The MC simulation models developed in this work are limited in their ability to mimic reality. Regarding the cellular elemental compositions considered herein, none are based on human data. Given the sensitivity of cell-level energy deposition to cellular elemental compositions for kilovoltage photon energies (see e.g., chapter 3), it can be concluded that there is a need for additional data on human cellular media. Furthermore, bulk tissue elemental composition and density data are often based on a small number of tissue samples; thus there is a need for bulk tissue media definitions that are more representative of a larger population.

Energy deposition within cells is also strongly dependent on cell morphology, suggesting that cell morphology measurements for normal and malignant human tissues is 
an area for continued development. The literature survey presented in table 2.2 provides a database of human cell and nucleus sizes relevant for future studies considering energy deposition on the cellular level. Furthermore, while a spherical cell model was employed in this work, human cells exist in a variety of shapes. ${ }^{95}$

This work focuses on scoring energy deposition within cell nuclei and cytoplasms. However, there are a variety of biologically-relevant targets spanning different length scales: e.g., mitochondria, cell membranes, constituents of neighbouring cells, water molecules associated with DNA. A more realistic, non-homogeneous model of the cytoplasm would include explicitly-modelled organelles such as mitochondria suspended within the cytosol. A more realistic model of the ECM would involve a complex network of proteins such as collagen — the composition of this network varies with tissue type. ${ }^{175}$ Furthermore, chromatin condensation and dispersion occurs throughout the cell cycle, implying an ever-changing DNA target size. ${ }^{10}$ Recently, highly complex models of DNA, capable of including detail on the atomic level, have been developed for use with the Geant4 MC software. ${ }^{176}$ Future work may involve development of more complex microscopic tissue structure models, allowing investigation of energy deposition within these other targets. In this thesis, the EGSnrc MC software has been used to perform simulations of radiation transport and energy deposition on the cellular level. The $1 \mathrm{keV}$ transport cutoff and production threshold for electrons and photons is expected to be adequate for the simulation geometries considered herein given that the CSDA range of a $1 \mathrm{keV}$ electron in water is $\sim 40 \mathrm{~nm}$. Furthermore, EGSnrc has been found to be robust to changes in transport parameters (appendix A), and has been validated by comparison with other MC codes, considering macro- and microscopic scale simulations. ${ }^{177,178}$ However, if targets on the nanometer scale or on the molecular level are of interest, then track structure codes may need to be considered. ${ }^{133,179}$

For the cell population models considered herein, a limited number of 'microstates' (multicellular models) corresponding to a given 'macrostate' (bulk tissue composition 
and density) were considered. In reality, the true 'microstate' is unknown, and there are an extensive number of possible 'microstates'. Results presented in chapter 5 demonstrate that randomly sampling cell and nucleus sizes from a normal distribution will lead to larger microdosimetric spread than if the cells are modelled in a less realistic manner, with cell and nucleus sizes constant throughout the cell population. Size distribution method is therefore an example of a microstate parameter that influences relevant dosimetric quantities, whereas cell arrangement method has considerably less influence. Only a single cell number density was considered in this work. However, Backman et al. ${ }^{104}$ report that there is a higher density of cells in regions of dysplasia or carcinoma, due to the unregulated proliferation of abnormal cells. In chapters 5 and 7 , cell and nuclear radii are randomly sampled from a normal distribution with a standard deviation of $1 \mathrm{\mu m}$, which is motivated by breast cancer cell data taken from Cai et $a l .{ }^{102}$ However, Perelman et al. ${ }^{112}$ show that dysplastic or malignant cells demonstrate more variation in nuclear size than normal cells. The results of Perelman et al. ${ }^{112}$ also support the general trend that nucleus size relative to cell size is larger for malignant cells than for healthy cells. ${ }^{96,104}$ Future work may consider the dosimetric consequences of these differences between normal and malignant cells in more detail, and may explore the microstate parameter space more thoroughly to determine which parameters influence energy deposition within subcellular targets.

The work presented in this thesis has implications for existing, emerging and prospective radiotherapy treatments and diagnostic radiology techniques. For existing treatments, the goal is to develop a better understanding of energy deposition on the cellular level, which may lead to more accurate treatment planning algorithms, and improved understanding of treatment outcomes for retrospective assessment. Cellular dosimetry can be used to assess the risks and potential benefits of prospective and emerging treatments such as gold nanoparticle radiosensitization, ${ }^{178}$ leading to the development of safer and more effective procedures. Consideration of cellular doses in 
diagnostic radiology risk models may lead to better informed risk assessment.

Furthermore, fit parameters for an equation relating the relative standard deviation of the specific energy to the macroscopic dose and the target volume are presented in chapters 5 and 6 - these equations provide researchers with convenient estimates of the microdosimetric spread in a variety of scenarios. In addition to Raman spectroscopy measurements of cell response to ionizing radiation (chapter 6) and diagnostic radiology risk assessment contexts such as mammography (chapter 7), these estimates may also be useful for other radiation response or dosimetry studies using microscopic sampling regions; for example, $\mu \mathrm{m}$-sized MOSFET dosimeters have been developed for in vivo dosimetry. ${ }^{180}$ However, MC-calculated values of the microdosimetric spread will underestimate the true variation due to uncertainty in the experimental setup; for radiation response studies, biological effects will also contribute to the variation.

Throughout this thesis, multiscale computational models, which combine varying levels of detail on different length scales, are used. It is likely that the future of computational radiation dosimetry for medical physics will involve a multiscale modelling framework, which will allow for calculations of energy deposition on microscopic length scales, without having to model microscopic details throughout an entire macroscopic region. To achieve accurate scatter conditions while also ensuring that the simulation is computationally feasible, microscopic detail is included only in the vicinity of the target of interest. Only physical effects (e.g., ionizations, excitations, scattering) are considered in this work. However, chemical (e.g., free radical production, diffusion) and biological effects (e.g., DNA damage and repair, cell signalling, cell death) also exist. ${ }^{58}$ A more complete multiscale modelling framework may consider various phenomena occurring on a range of temporal and spatial scales. 


\section{Appendix A}

\section{Transport parameter tests}

To investigate sensitivity of MC simulation results with respect to transport and variance reduction parameters, additional simulations involving the geometries used in chapter 3 (see figure 3.1) are carried out, changing one transport or variance reduction parameter at a time. Two arbitrary test cases are considered: (1) adenoidcystic carcinoma as the bulk tissue with $50 \mathrm{keV}$ incident photons, and (2) squamous cell lung carcinoma as the bulk tissue with $20 \mathrm{keV}$ incident photons. Results are summarized in figure A.1. The effect of changing the following parameters is considered (the abbreviations used in figure A.1 are shown in brackets):

- Brems cross sections ('BCS')) = BH database instead of NRC database

- Skin depth for BCA ('skin depth') $=5,10,100,1 \times 10^{10}$ instead of 3 , in units of the electron mean free path

- ESTEPE $=0.1$ instead of 0.25

- $\quad$ ECUT and PCUT ('E/PCUT') = 2, 3, $5 \mathrm{keV}$ instead of $1 \mathrm{keV}$

- $\quad$ CSE factor ('CSE') = 1028, 256 instead of 4096

- Radius for $\mathrm{CSE}=0.06 \mathrm{~cm}$ instead of $0.006 \mathrm{~cm}$ for the $20 \mathrm{keV}$ case; $R_{C S E}=0.2 \mathrm{~cm}$ instead of $0.02 \mathrm{~cm}$ for the $50 \mathrm{keV}$ case (' $R_{C S E} \times 10^{\prime}$ ) 
- Radiative Compton corrections ('rad. Compton') = On instead of Off

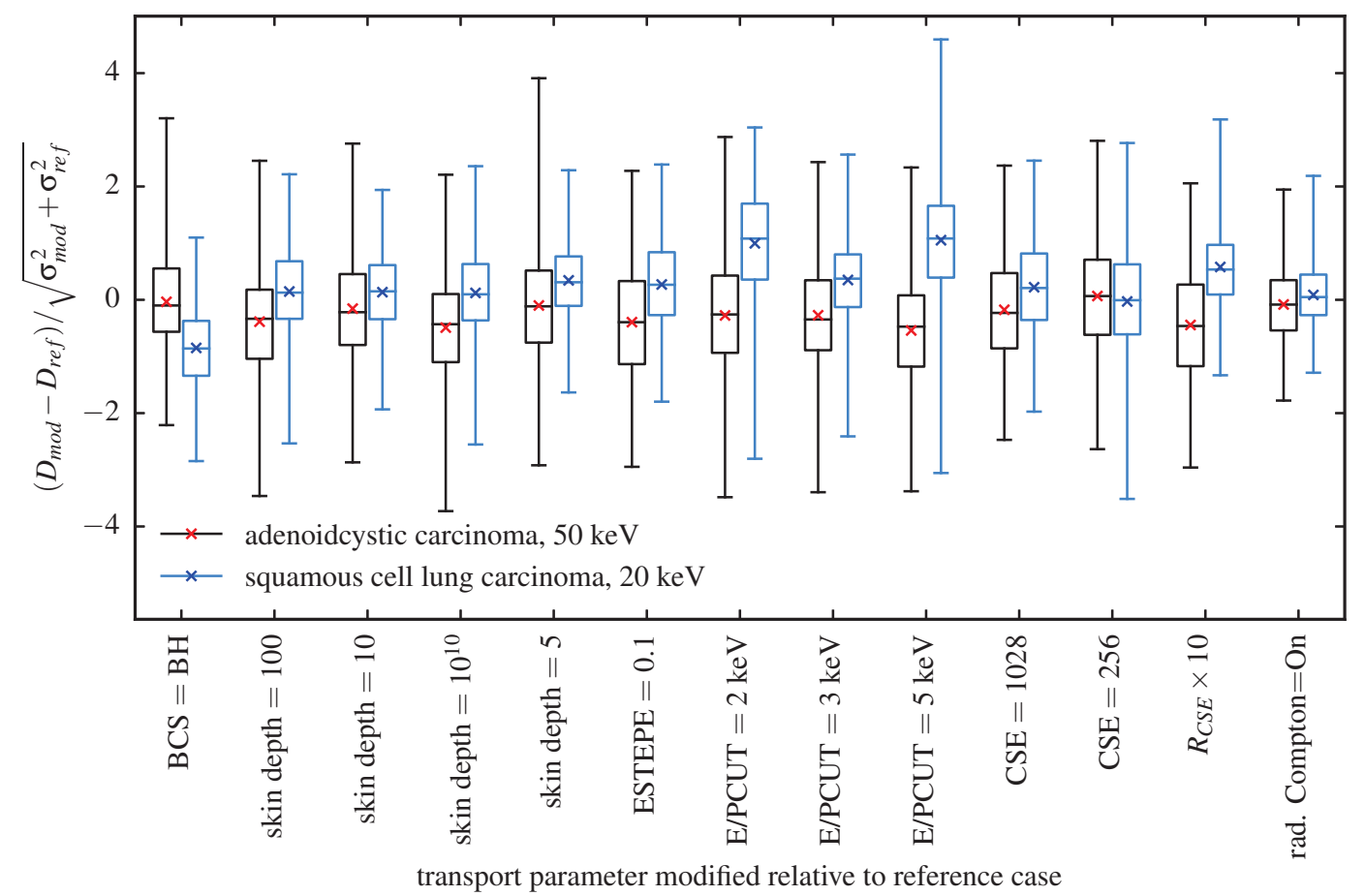

Figure A.1: Transport and variance reduction parameter sensitivity test results for two example cases: adenoidcystic carcinoma at $50 \mathrm{keV}$, and squamous cell lung carcinoma at $20 \mathrm{keV}$. Abbreviations used for the horizontal axis labels are described in the text. $D_{\bmod }$ is the dose in the case where one of the transport/variance reduction parameters has been changed as indicated on the horizontal axis, and $\sigma_{m o d}$ is its corresponding uncertainty. $D_{r e f}$ is the dose in the reference case, which uses the transport parameters described in chapter 2, and $\sigma_{r e f}$ is its corresponding uncertainty. Whiskers extend between the minimum and maximum values of the set of results for the nine cell/nucleus size combinations and seven cytoplasm/nucleus elemental compositions for cytoplasm and nuclear targets, for the geometries shown in figure 3.1; box extends between the upper and lower quartiles; its horizontal line indicates the median, and the ' $\mathrm{x}$ ' symbol indicates the mean value.

A brief description of the parameters considered in figure A.1 in given here; see Kawrakow et al. ${ }^{28}$ for more details. Skin depth for BCA is the distance from a boundary where the transport algorithm will switch to the boundary crossing algorithm (BCA). For all of the simulations presented in this thesis, BCA is set to 'EXACT', meaning that single scattering mode is used to cross boundaries (condensed history is turned off). ESTEPE is the maximum fractional energy loss per step. ECUT and PCUT are 
the electron and photon transport cutoffs, respectively. Electrons (photons) with energy less than ECUT (PCUT) will have their histories terminated and their energy deposited locally. The CSE factor is the cross section enhancement factor, which is applied within a spherical region of radius $R_{C S E}$ surrounding the 13-cell cluster. Radiative Compton corrections account for the possibility that Compton electrons may emit and reabsorb virtual photons, which can affect the Compton scattering cross section. 


\section{Appendix B}

\section{Supplementary material for chapter 5}

Results are summarized in the tables below for melanoma, muscle and mammary gland, for six incident photon energies, and six cell/nucleus size combinations. Results are presented for nuclear targets, for 'random sizes; random arrangement': $D_{m, m}$ and cor-

responding statistical uncertainty ('unc.'), fraction of nuclei receiving no energy deposition, RMSE for MC-calculated $f(z, D)$ compared to a normal distribution with the same mean and standard deviation, relative standard deviation of the specific energy (MC) and percent discrepancy of this value with the fit according to equation 2 with parameters in table $2, \% \Delta_{f i t, M C}=\left[\left(\sigma_{z} / \bar{z}\right)_{f i t}-\left(\sigma_{z} / \bar{z}\right)_{M C}\right] /\left(\sigma_{z} / \bar{z}\right)_{M C} \times 100 \%$. 
Table B.1: Summary of $20 \mathrm{keV}$ results for melanoma.

\begin{tabular}{|c|c|c|c|c|c|c|}
\hline \multirow{2}{*}{$\left(r_{c e l l}, r_{n u c}\right)$} & \multicolumn{2}{|c|}{$D_{m, m}$} & \multicolumn{2}{|c|}{$f(z)$} & \multicolumn{2}{|c|}{$\sigma_{z} / \bar{z}$} \\
\hline & mGy & $\%$ unc. & $f(z=0)$ & RMSE & $\mathrm{MC} \times 100 \%$ & $\% \Delta_{f i t, M C}$ \\
\hline \multirow{4}{*}{$(5,2) \mu \mathrm{m}$} & 1.5 & 2.6 & 0.90 & 0.15 & 601 & 17 \\
\hline & 5.5 & 1.3 & 0.76 & 0.11 & 309 & 17 \\
\hline & 10.3 & 1.0 & 0.64 & 0.079 & 227 & 17 \\
\hline & 50.3 & 0.4 & 0.26 & 0.018 & 127 & -6 \\
\hline \multirow{4}{*}{$(5,4) \mu \mathrm{m}$} & 1.5 & 2.6 & 0.82 & 0.19 & 386 & -9 \\
\hline & 5.5 & 1.3 & 0.50 & 0.10 & 179 & 1 \\
\hline & 10.3 & 1.0 & 0.32 & 0.047 & 139 & -5 \\
\hline & 50.3 & 0.4 & 0.033 & 0.0043 & 62 & -4 \\
\hline \multirow{4}{*}{$(7.5,3) \mu \mathrm{m}$} & 1.5 & 1.5 & 0.84 & 0.21 & 336 & 17 \\
\hline & 5.7 & 0.8 & 0.57 & 0.084 & 294 & -31 \\
\hline & 10.4 & 0.6 & 0.39 & 0.046 & 250 & -40 \\
\hline & 50.1 & 0.3 & 0.063 & 0.0061 & 80 & -15 \\
\hline \multirow{4}{*}{$(7.5,6) \mu \mathrm{m}$} & 1.5 & 1.5 & 0.48 & 0.17 & 153 & -6 \\
\hline & 5.7 & 0.8 & 0.092 & 0.034 & 81 & -8 \\
\hline & 10.4 & 0.6 & 0.018 & 0.0089 & 57 & -3 \\
\hline & 50.1 & 0.3 & 0.00 & 0.0037 & 26 & -5 \\
\hline \multirow{4}{*}{$(10,4) \mu \mathrm{m}$} & 1.5 & 1.1 & 0.73 & 0.19 & 289 & -13 \\
\hline & 5.5 & 0.6 & 0.35 & 0.076 & 142 & -8 \\
\hline & 10.4 & 0.4 & 0.18 & 0.035 & 100 & -5 \\
\hline & 50.1 & 0.2 & 0.0078 & 0.0041 & 47 & -7 \\
\hline \multirow{4}{*}{$(10,8) \mu \mathrm{m}$} & 1.5 & 1.1 & 0.21 & 0.10 & 100 & -16 \\
\hline & 5.5 & 0.6 & 0.010 & 0.012 & 53 & -17 \\
\hline & 10.4 & 0.4 & 0.00 & 0.0076 & 39 & -19 \\
\hline & 50.1 & 0.2 & 0.00 & 0.0055 & 17 & -17 \\
\hline
\end{tabular}


Table B.2: Summary of $30 \mathrm{keV}$ results for melanoma.

\begin{tabular}{|c|c|c|c|c|c|c|}
\hline \multirow{2}{*}{$\left(r_{\text {cell }}, r_{\text {nuc }}\right)$} & \multicolumn{2}{|c|}{$D_{m, m}$} & \multicolumn{2}{|c|}{$f(z)$} & \multicolumn{2}{|c|}{$\sigma_{z} / \bar{z}$} \\
\hline & mGy & $\%$ unc. & $f(z=0)$ & RMSE & $\mathrm{MC} \times 100 \%$ & $\% \Delta_{f i t, M C}$ \\
\hline \multirow{4}{*}{$(5,2) \mu \mathrm{m}$} & 1.2 & 3.5 & 0.92 & 0.12 & 993 & -26 \\
\hline & 5.1 & 1.7 & 0.73 & 0.088 & 407 & -13 \\
\hline & 10.1 & 1.2 & 0.56 & 0.053 & 290 & -13 \\
\hline & 50.3 & 0.5 & 0.16 & 0.011 & 112 & 2 \\
\hline \multirow{4}{*}{$(5,4) \mu \mathrm{m}$} & 1.2 & 3.5 & 0.79 & 0.19 & 349 & 9 \\
\hline & 5.1 & 1.7 & 0.42 & 0.079 & 166 & 10 \\
\hline & 10.1 & 1.2 & 0.24 & 0.037 & 117 & 12 \\
\hline & 50.3 & 0.5 & 0.021 & 0.0035 & 59 & -1 \\
\hline \multirow{4}{*}{$(7.5,3) \mu \mathrm{m}$} & 1.2 & 2.1 & 0.84 & 0.17 & 482 & -10 \\
\hline & 5.1 & 1.0 & 0.50 & 0.083 & 213 & -2 \\
\hline & 10.3 & 0.7 & 0.31 & 0.041 & 167 & -13 \\
\hline & 50.1 & 0.3 & 0.047 & 0.0054 & 70 & -5 \\
\hline \multirow{4}{*}{$(7.5,6) \mu \mathrm{m}$} & 1.2 & 2.1 & 0.51 & 0.20 & 174 & -4 \\
\hline & 5.1 & 1.0 & 0.071 & 0.031 & 78 & 3 \\
\hline & 10.3 & 0.7 & 0.0086 & 0.0081 & 56 & 1 \\
\hline & 50.1 & 0.3 & 0.00 & 0.0037 & 25 & 2 \\
\hline \multirow{4}{*}{$(10,4) \mu \mathrm{m}$} & 1.2 & 1.5 & 0.75 & 0.23 & 278 & 2 \\
\hline & 5.3 & 0.7 & 0.31 & 0.071 & 123 & 7 \\
\hline & 10.2 & 0.5 & 0.14 & 0.026 & 95 & 1 \\
\hline & 50.1 & 0.2 & 0.0059 & 0.0036 & 41 & 4 \\
\hline \multirow{4}{*}{$(10,8) \mu \mathrm{m}$} & 1.2 & 1.5 & 0.21 & 0.15 & 117 & -16 \\
\hline & 5.3 & 0.7 & 0.00 & 0.013 & 52 & -12 \\
\hline & 10.2 & 0.5 & 0.00 & 0.010 & 38 & -11 \\
\hline & 50.1 & 0.2 & 0.00 & 0.0039 & 17 & -9 \\
\hline
\end{tabular}


Table B.3: Summary of $50 \mathrm{keV}$ results for melanoma.

\begin{tabular}{|c|c|c|c|c|c|c|}
\hline \multirow{2}{*}{$\left(r_{\text {cell }}, r_{\text {nuc }}\right)$} & \multicolumn{2}{|c|}{$D_{m, m}$} & \multicolumn{2}{|c|}{$f(z)$} & \multicolumn{2}{|c|}{$\sigma_{z} / \bar{z}$} \\
\hline & mGy & $\%$ unc. & $f(z=0)$ & RMSE & $\mathrm{MC} \times 100 \%$ & $\% \Delta_{f i t, M C}$ \\
\hline \multirow{4}{*}{$(5,2) \mu \mathrm{m}$} & 1.0 & 4.0 & 0.91 & 0.19 & 529 & 40 \\
\hline & 7.9 & 1.5 & 0.55 & 0.074 & 206 & 30 \\
\hline & 10.5 & 1.3 & 0.47 & 0.057 & 196 & 18 \\
\hline & 51.0 & 0.6 & 0.14 & 0.010 & 109 & -4 \\
\hline \multirow{4}{*}{$(5,4) \mu \mathrm{m}$} & 1.0 & 4.0 & 0.77 & 0.22 & 261 & 39 \\
\hline & 7.9 & 1.5 & 0.22 & 0.039 & 111 & 19 \\
\hline & 10.5 & 1.3 & 0.15 & 0.024 & 99 & 16 \\
\hline & 51.0 & 0.6 & 0.010 & 0.0033 & 46 & 13 \\
\hline \multirow{4}{*}{$(7.5,3) \mu \mathrm{m}$} & 1.0 & 2.5 & 0.81 & 0.21 & 383 & 10 \\
\hline & 7.8 & 0.9 & 0.31 & 0.053 & 127 & 19 \\
\hline & 10.5 & 0.8 & 0.25 & 0.035 & 144 & -9 \\
\hline & 50.7 & 0.4 & 0.032 & 0.0042 & 62 & -5 \\
\hline \multirow{4}{*}{$(7.5,6) \mu \mathrm{m}$} & 1.0 & 2.5 & 0.45 & 0.17 & 148 & 3 \\
\hline & 7.8 & 0.9 & 0.017 & 0.011 & 57 & -5 \\
\hline & 10.5 & 0.8 & 0.010 & 0.010 & 50 & -6 \\
\hline & 50.7 & 0.4 & 0.00 & 0.0038 & 22 & -3 \\
\hline \multirow{4}{*}{$(10,4) \mu \mathrm{m}$} & 1.0 & 1.7 & 0.69 & 0.18 & 316 & -17 \\
\hline & 7.8 & 0.6 & 0.12 & 0.025 & 95 & 0 \\
\hline & 10.4 & 0.5 & 0.082 & 0.016 & 82 & 1 \\
\hline & 50.7 & 0.2 & 0.0039 & 0.0035 & 36 & 4 \\
\hline \multirow{4}{*}{$(10,8) \mu \mathrm{m}$} & 1.0 & 1.7 & 0.17 & 0.15 & 107 & -20 \\
\hline & 7.8 & 0.6 & 0.00 & 0.012 & 37 & -16 \\
\hline & 10.4 & 0.5 & 0.00 & 0.0095 & 32 & -17 \\
\hline & 50.7 & 0.2 & 0.00 & 0.0049 & 15 & -18 \\
\hline
\end{tabular}


Table B.4: Summary of $90 \mathrm{keV}$ results for melanoma.

\begin{tabular}{|c|c|c|c|c|c|c|}
\hline \multirow{2}{*}{$\left(r_{\text {cell }}, r_{\text {nuc }}\right)$} & \multicolumn{2}{|c|}{$D_{m, m}$} & \multicolumn{2}{|c|}{$f(z)$} & \multicolumn{2}{|c|}{$\sigma_{z} / \bar{z}$} \\
\hline & mGy & $\%$ unc. & $f(z=0)$ & RMSE & $\mathrm{MC} \times 100 \%$ & $\% \Delta_{f i t, M C}$ \\
\hline \multirow{4}{*}{$(5,2) \mu \mathrm{m}$} & 1.0 & 3.7 & 0.94 & 0.18 & 771 & 10 \\
\hline & 5.7 & 1.6 & 0.70 & 0.082 & 351 & 1 \\
\hline & 10.4 & 1.1 & 0.55 & 0.060 & 242 & 9 \\
\hline & 50.0 & 0.5 & 0.18 & 0.012 & 111 & 9 \\
\hline \multirow{4}{*}{$(5,4) \mu \mathrm{m}$} & 1.0 & 3.7 & 0.84 & 0.23 & 370 & 8 \\
\hline & 5.7 & 1.6 & 0.39 & 0.074 & 154 & 9 \\
\hline & 10.4 & 1.1 & 0.21 & 0.033 & 111 & 13 \\
\hline & 50.0 & 0.5 & 0.023 & 0.0036 & 53 & 8 \\
\hline \multirow{4}{*}{$(7.5,3) \mu \mathrm{m}$} & 1.0 & 2.2 & 0.81 & 0.21 & 327 & 41 \\
\hline & 5.6 & 0.9 & 0.44 & 0.075 & 163 & 21 \\
\hline & 10.3 & 0.7 & 0.27 & 0.032 & 199 & -27 \\
\hline & 50.8 & 0.3 & 0.041 & 0.0047 & 72 & -9 \\
\hline \multirow{4}{*}{$(7.5,6) \mu \mathrm{m}$} & 1.0 & 2.2 & 0.54 & 0.19 & 160 & -1 \\
\hline & 5.6 & 0.9 & 0.029 & 0.017 & 67 & 0 \\
\hline & 10.3 & 0.7 & 0.0038 & 0.0092 & 52 & -5 \\
\hline & 50.8 & 0.3 & 0.00 & 0.0035 & 24 & -7 \\
\hline \multirow{4}{*}{$(10,4) \mu \mathrm{m}$} & 1.0 & 1.6 & 0.70 & 0.19 & 280 & 1 \\
\hline & 5.8 & 0.7 & 0.24 & 0.048 & 126 & -6 \\
\hline & 10.5 & 0.5 & 0.10 & 0.018 & 91 & -3 \\
\hline & 50.8 & 0.2 & 0.0039 & 0.0032 & 38 & 6 \\
\hline \multirow{4}{*}{$(10,8) \mu \mathrm{m}$} & 1.0 & 1.6 & 0.23 & 0.11 & 106 & -18 \\
\hline & 5.8 & 0.7 & 0.0020 & 0.017 & 44 & -17 \\
\hline & 10.5 & 0.5 & 0.00 & 0.010 & 31 & -14 \\
\hline & 50.8 & 0.2 & 0.00 & 0.0046 & 14 & -11 \\
\hline
\end{tabular}


Table B.5: Summary of $370 \mathrm{keV}$ results for melanoma.

\begin{tabular}{|c|c|c|c|c|c|c|}
\hline \multirow{2}{*}{$\left(r_{\text {cell }}, r_{\text {nuc }}\right)$} & \multicolumn{2}{|c|}{$D_{m, m}$} & \multicolumn{2}{|c|}{$f(z)$} & \multicolumn{2}{|c|}{$\sigma_{z} / \bar{z}$} \\
\hline & mGy & $\%$ unc. & $f(z=0)$ & RMSE & $\mathrm{MC} \times 100 \%$ & $\% \Delta_{f i t, M C}$ \\
\hline \multirow{4}{*}{$(5,2) \mu \mathrm{m}$} & 1.0 & 5.7 & 0.84 & 0.16 & 576 & 1 \\
\hline & 5.1 & 2.6 & 0.49 & 0.074 & 251 & 3 \\
\hline & 10.1 & 1.8 & 0.31 & 0.036 & 205 & -10 \\
\hline & 50.6 & 0.8 & 0.055 & 0.0052 & 79 & 4 \\
\hline \multirow{4}{*}{$(5,4) \mu \mathrm{m}$} & 1.0 & 5.7 & 0.63 & 0.19 & 245 & 21 \\
\hline & 5.1 & 2.6 & 0.19 & 0.044 & 109 & 22 \\
\hline & 10.1 & 1.8 & 0.061 & 0.015 & 82 & 15 \\
\hline & 50.6 & 0.8 & 0.0019 & 0.0046 & 41 & 2 \\
\hline \multirow{4}{*}{$(7.5,3) \mu \mathrm{m}$} & 1.0 & 3.8 & 0.69 & 0.22 & 279 & 20 \\
\hline & 5.0 & 1.7 & 0.25 & 0.054 & 135 & 13 \\
\hline & 10.0 & 1.2 & 0.10 & 0.019 & 95 & 14 \\
\hline & 50.0 & 0.5 & 0.0058 & 0.0042 & 47 & 2 \\
\hline \multirow{4}{*}{$(7.5,6) \mu \mathrm{m}$} & 1.0 & 3.8 & 0.32 & 0.16 & 135 & -4 \\
\hline & 5.0 & 1.7 & 0.0086 & 0.020 & 61 & -5 \\
\hline & 10.0 & 1.2 & 0.00 & 0.011 & 43 & -4 \\
\hline & 50.0 & 0.5 & 0.00 & 0.0038 & 19 & -1 \\
\hline \multirow{4}{*}{$(10,4) \mu \mathrm{m}$} & 1.0 & 2.8 & 0.53 & 0.19 & 195 & 12 \\
\hline & 5.1 & 1.3 & 0.092 & 0.033 & 94 & 5 \\
\hline & 10.0 & 0.9 & 0.026 & 0.012 & 69 & 1 \\
\hline & 51.0 & 0.4 & 0.0010 & 0.0038 & 29 & 5 \\
\hline \multirow{4}{*}{$(10,8) \mu \mathrm{m}$} & 1.0 & 2.8 & 0.10 & 0.13 & 92 & -17 \\
\hline & 5.1 & 1.3 & 0.00 & 0.018 & 42 & -18 \\
\hline & 10.0 & 0.9 & 0.00 & 0.0094 & 30 & -19 \\
\hline & 51.0 & 0.4 & 0.00 & 0.0045 & 13 & -20 \\
\hline
\end{tabular}


Table B.6: Summary of ${ }^{60}$ Co results for melanoma.

\begin{tabular}{|c|c|c|c|c|c|c|}
\hline \multirow{2}{*}{$\left(r_{\text {cell }}, r_{\text {nuc }}\right)$} & \multicolumn{2}{|c|}{$D_{m, m}$} & \multicolumn{2}{|c|}{$f(z)$} & \multicolumn{2}{|c|}{$\sigma_{z} / \bar{z}$} \\
\hline & mGy & $\%$ unc. & $f(z=0)$ & RMSE & $\mathrm{MC} \times 100 \%$ & $\% \Delta_{f i t, M C}$ \\
\hline \multirow{4}{*}{$(5,2) \mu \mathrm{m}$} & 1.2 & 4.5 & 0.69 & 0.20 & 280 & 48 \\
\hline & 5.2 & 2.2 & 0.29 & 0.056 & 171 & 16 \\
\hline & 10.1 & 1.5 & 0.15 & 0.024 & 126 & 13 \\
\hline & 50.7 & 0.7 & 0.010 & 0.0045 & 66 & -4 \\
\hline \multirow{4}{*}{$(5,4) \mu \mathrm{m}$} & 1.2 & 4.5 & 0.39 & 0.17 & 216 & 2 \\
\hline & 5.2 & 2.2 & 0.044 & 0.035 & 95 & 11 \\
\hline & 10.1 & 1.5 & 0.015 & 0.016 & 66 & 15 \\
\hline & 50.7 & 0.7 & 0.0029 & 0.0040 & 32 & 7 \\
\hline \multirow{4}{*}{$(7.5,3) \mu \mathrm{m}$} & 1.0 & 3.4 & 0.51 & 0.18 & 355 & -24 \\
\hline & 5.1 & 1.5 & 0.10 & 0.040 & 122 & -2 \\
\hline & 10.2 & 1.1 & 0.036 & 0.019 & 84 & 1 \\
\hline & 51.1 & 0.5 & 0.0019 & 0.0046 & 39 & -3 \\
\hline \multirow{4}{*}{$(7.5,6) \mu \mathrm{m}$} & 1.0 & 3.4 & 0.14 & 0.17 & 104 & 4 \\
\hline & 5.1 & 1.5 & 0.0010 & 0.028 & 47 & 2 \\
\hline & 10.2 & 1.1 & 0.00 & 0.014 & 33 & 4 \\
\hline & 51.1 & 0.5 & 0.00 & 0.0043 & 15 & 3 \\
\hline \multirow{4}{*}{$(10,4) \mu \mathrm{m}$} & 1.0 & 2.6 & 0.30 & 0.19 & 157 & 13 \\
\hline & 5.0 & 1.2 & 0.024 & 0.036 & 80 & -1 \\
\hline & 10.1 & 0.8 & 0.0039 & 0.019 & 61 & -8 \\
\hline & 51.3 & 0.4 & 0.00 & 0.0043 & 24 & 3 \\
\hline \multirow{4}{*}{$(10,8) \mu \mathrm{m}$} & 1.0 & 2.6 & 0.013 & 0.10 & 72 & -9 \\
\hline & 5.0 & 1.2 & 0.00 & 0.016 & 33 & -12 \\
\hline & 10.1 & 0.8 & 0.00 & 0.011 & 24 & -14 \\
\hline & 51.3 & 0.4 & 0.00 & 0.0050 & 10 & -13 \\
\hline
\end{tabular}


Table B.7: Summary of $20 \mathrm{keV}$ results for muscle.

\begin{tabular}{|c|c|c|c|c|c|c|}
\hline \multirow{2}{*}{$\left(r_{\text {cell }}, r_{\text {nuc }}\right)$} & \multicolumn{2}{|c|}{$D_{m, m}$} & \multicolumn{2}{|c|}{$f(z)$} & \multicolumn{2}{|c|}{$\sigma_{z} / \bar{z}$} \\
\hline & mGy & $\%$ unc. & $f(z=0)$ & RMSE & $\mathrm{MC} \times 100 \%$ & $\% \Delta_{f i t, M C}$ \\
\hline \multirow{4}{*}{$(5,2) \mu \mathrm{m}$} & 1.0 & 3.1 & 0.94 & 0.21 & 704 & 19 \\
\hline & 5.0 & 1.4 & 0.76 & 0.10 & 334 & 14 \\
\hline & 10.0 & 1.0 & 0.61 & 0.062 & 275 & -2 \\
\hline & 50.3 & 0.4 & 0.24 & 0.017 & 113 & 6 \\
\hline \multirow{4}{*}{$(5,4) \mu \mathrm{m}$} & 1.0 & 3.1 & 0.84 & 0.18 & 471 & -11 \\
\hline & 5.0 & 1.4 & 0.47 & 0.085 & 180 & 5 \\
\hline & 10.0 & 1.0 & 0.28 & 0.044 & 125 & 7 \\
\hline & 50.3 & 0.4 & 0.033 & 0.0040 & 56 & 6 \\
\hline \multirow{4}{*}{$(7.5,3) \mu \mathrm{m}$} & 1.0 & 1.9 & 0.89 & 0.23 & 434 & 10 \\
\hline & 5.0 & 0.8 & 0.57 & 0.10 & 196 & 10 \\
\hline & 10.0 & 0.6 & 0.37 & 0.055 & 138 & 11 \\
\hline & 50.3 & 0.3 & 0.057 & 0.0053 & 70 & -3 \\
\hline \multirow{4}{*}{$(7.5,6) \mu \mathrm{m}$} & 1.0 & 1.9 & 0.58 & 0.20 & 189 & -7 \\
\hline & 5.0 & 0.8 & 0.11 & 0.037 & 83 & -4 \\
\hline & 10.0 & 0.6 & 0.022 & 0.0073 & 59 & -4 \\
\hline & 50.3 & 0.3 & 0.00 & 0.0036 & 26 & -2 \\
\hline \multirow{4}{*}{$(10,4) \mu \mathrm{m}$} & 1.0 & 1.3 & 0.78 & 0.23 & 285 & 7 \\
\hline & 5.1 & 0.6 & 0.34 & 0.076 & 131 & 4 \\
\hline & 10.0 & 0.4 & 0.16 & 0.029 & 97 & 1 \\
\hline & 50.3 & 0.2 & 0.0049 & 0.0029 & 43 & 2 \\
\hline \multirow{4}{*}{$(10,8) \mu \mathrm{m}$} & 1.0 & 1.3 & 0.27 & 0.11 & 110 & -8 \\
\hline & 5.1 & 0.6 & 0.0059 & 0.015 & 50 & -10 \\
\hline & 10.0 & 0.4 & 0.00 & 0.0056 & 36 & -11 \\
\hline & 50.3 & 0.2 & 0.00 & 0.0062 & 16 & -8 \\
\hline
\end{tabular}


Table B.8: Summary of $30 \mathrm{keV}$ results for muscle.

\begin{tabular}{|c|c|c|c|c|c|c|}
\hline \multirow{2}{*}{$\left(r_{c e l l}, r_{n u c}\right)$} & \multicolumn{2}{|c|}{$D_{m, m}$} & \multicolumn{2}{|c|}{$f(z)$} & \multicolumn{2}{|c|}{$\sigma_{z} / \bar{z}$} \\
\hline & mGy & $\%$ unc. & $f(z=0)$ & RMSE & $\mathrm{MC} \times 100 \%$ & $\% \Delta_{f i t, M C}$ \\
\hline \multirow{4}{*}{$(5,2) \mu \mathrm{m}$} & 1.2 & 3.4 & 0.92 & 0.19 & 659 & 11 \\
\hline & 5.2 & 1.6 & 0.72 & 0.10 & 284 & 24 \\
\hline & 10.0 & 1.2 & 0.57 & 0.070 & 216 & 18 \\
\hline & 50.2 & 0.5 & 0.20 & 0.014 & 105 & 8 \\
\hline \multirow{4}{*}{$(5,4) \mu \mathrm{m}$} & 1.2 & 3.4 & 0.78 & 0.20 & 300 & 26 \\
\hline & 5.2 & 1.6 & 0.41 & 0.083 & 149 & 22 \\
\hline & 10.0 & 1.2 & 0.23 & 0.036 & 111 & 18 \\
\hline & 50.2 & 0.5 & 0.029 & 0.0040 & 56 & 4 \\
\hline \multirow{4}{*}{$(7.5,3) \mu \mathrm{m}$} & 1.3 & 2.0 & 0.82 & 0.20 & 335 & 25 \\
\hline & 5.3 & 1.0 & 0.48 & 0.086 & 179 & 14 \\
\hline & 10.0 & 0.7 & 0.30 & 0.043 & 135 & 9 \\
\hline & 50.1 & 0.3 & 0.052 & 0.0052 & 63 & 6 \\
\hline \multirow{4}{*}{$(7.5,6) \mu \mathrm{m}$} & 1.3 & 2.0 & 0.47 & 0.18 & 164 & -1 \\
\hline & 5.3 & 1.0 & 0.058 & 0.028 & 80 & -2 \\
\hline & 10.0 & 0.7 & 0.0086 & 0.011 & 59 & -4 \\
\hline & 50.1 & 0.3 & 0.00 & 0.0041 & 26 & -2 \\
\hline \multirow{4}{*}{$(10,4) \mu \mathrm{m}$} & 1.3 & 1.4 & 0.71 & 0.21 & 271 & 0 \\
\hline & 5.2 & 0.7 & 0.28 & 0.063 & 121 & 10 \\
\hline & 10.2 & 0.5 & 0.12 & 0.021 & 87 & 9 \\
\hline & 50.1 & 0.2 & 0.0039 & 0.0036 & 41 & 5 \\
\hline \multirow{4}{*}{$(10,8) \mu \mathrm{m}$} & 1.3 & 1.4 & 0.18 & 0.12 & 104 & -9 \\
\hline & 5.2 & 0.7 & 0.0039 & 0.013 & 52 & -11 \\
\hline & 10.2 & 0.5 & 0.0020 & 0.0094 & 39 & -14 \\
\hline & 50.1 & 0.2 & 0.00 & 0.0048 & 17 & -12 \\
\hline
\end{tabular}


Table B.9: Summary of $50 \mathrm{keV}$ results for muscle.

\begin{tabular}{|c|c|c|c|c|c|c|}
\hline \multirow{2}{*}{$\left(r_{\text {cell }}, r_{\text {nuc }}\right)$} & \multicolumn{2}{|c|}{$D_{m, m}$} & \multicolumn{2}{|c|}{$f(z)$} & \multicolumn{2}{|c|}{$\sigma_{z} / \bar{z}$} \\
\hline & mGy & $\%$ unc. & $f(z=0)$ & RMSE & $\mathrm{MC} \times 100 \%$ & $\% \Delta_{f i t, M C}$ \\
\hline \multirow{4}{*}{$(5,2) \mu \mathrm{m}$} & 1.1 & 3.9 & 0.90 & 0.18 & 544 & 31 \\
\hline & 5.1 & 1.8 & 0.66 & 0.095 & 268 & 24 \\
\hline & 10.1 & 1.3 & 0.51 & 0.050 & 295 & -20 \\
\hline & 50.0 & 0.6 & 0.16 & 0.011 & 122 & -13 \\
\hline \multirow{4}{*}{$(5,4) \mu \mathrm{m}$} & 1.1 & 3.9 & 0.79 & 0.20 & 381 & -8 \\
\hline & 5.1 & 1.8 & 0.36 & 0.071 & 158 & 3 \\
\hline & 10.1 & 1.3 & 0.18 & 0.029 & 105 & 11 \\
\hline & 50.0 & 0.6 & 0.015 & 0.0036 & 47 & 10 \\
\hline \multirow{4}{*}{$(7.5,3) \mu \mathrm{m}$} & 1.1 & 2.3 & 0.79 & 0.22 & 317 & 26 \\
\hline & 5.1 & 1.1 & 0.44 & 0.071 & 209 & -11 \\
\hline & 10.1 & 0.8 & 0.25 & 0.036 & 135 & -1 \\
\hline & 50.0 & 0.3 & 0.041 & 0.0046 & 55 & 8 \\
\hline \multirow{4}{*}{$(7.5,6) \mu \mathrm{m}$} & 1.1 & 2.3 & 0.41 & 0.16 & 147 & -2 \\
\hline & 5.1 & 1.1 & 0.036 & 0.022 & 71 & -5 \\
\hline & 10.1 & 0.8 & 0.0038 & 0.010 & 50 & -4 \\
\hline & 50.0 & 0.3 & 0.00 & 0.0045 & 23 & -5 \\
\hline \multirow{4}{*}{$(10,4) \mu \mathrm{m}$} & 1.1 & 1.6 & 0.66 & 0.20 & 218 & 15 \\
\hline & 5.1 & 0.8 & 0.22 & 0.048 & 107 & 10 \\
\hline & 10.0 & 0.5 & 0.081 & 0.016 & 80 & 5 \\
\hline & 50.0 & 0.2 & 0.0049 & 0.0036 & 37 & 1 \\
\hline \multirow{4}{*}{$(10,8) \mu \mathrm{m}$} & 1.1 & 1.6 & 0.13 & 0.13 & 100 & -18 \\
\hline & 5.1 & 0.8 & 0.00 & 0.020 & 47 & -18 \\
\hline & 10.0 & 0.5 & 0.00 & 0.012 & 34 & -20 \\
\hline & 50.0 & 0.2 & 0.00 & 0.0050 & 14 & -15 \\
\hline
\end{tabular}


Table B.10: Summary of $90 \mathrm{keV}$ results for muscle.

\begin{tabular}{|c|c|c|c|c|c|c|}
\hline \multirow{2}{*}{$\left(r_{\text {cell }}, r_{\text {nuc }}\right)$} & \multicolumn{2}{|c|}{$D_{m, m}$} & \multicolumn{2}{|c|}{$f(z)$} & \multicolumn{2}{|c|}{$\sigma_{z} / \bar{z}$} \\
\hline & mGy & $\%$ unc. & $f(z=0)$ & RMSE & $\mathrm{MC} \times 100 \%$ & $\% \Delta_{f i t, M C}$ \\
\hline \multirow{4}{*}{$(5,2) \mu \mathrm{m}$} & 1.1 & 3.5 & 0.92 & 0.20 & 727 & 13 \\
\hline & 5.0 & 1.6 & 0.69 & 0.10 & 287 & 32 \\
\hline & 10.2 & 1.1 & 0.55 & 0.070 & 204 & 30 \\
\hline & 50.4 & 0.5 & 0.18 & 0.012 & 125 & -4 \\
\hline \multirow{4}{*}{$(5,4) \mu \mathrm{m}$} & 1.1 & 3.5 & 0.83 & 0.20 & 431 & -10 \\
\hline & 5.0 & 1.6 & 0.44 & 0.083 & 175 & 3 \\
\hline & 10.2 & 1.1 & 0.25 & 0.039 & 119 & 6 \\
\hline & 50.4 & 0.5 & 0.028 & 0.0043 & 54 & 4 \\
\hline \multirow{4}{*}{$(7.5,3) \mu \mathrm{m}$} & 1.1 & 2.1 & 0.85 & 0.23 & 374 & 20 \\
\hline & 5.1 & 1.0 & 0.50 & 0.091 & 178 & 16 \\
\hline & 10.1 & 0.7 & 0.31 & 0.046 & 133 & 10 \\
\hline & 50.3 & 0.3 & 0.045 & 0.0049 & 64 & 1 \\
\hline \multirow{4}{*}{$(7.5,6) \mu \mathrm{m}$} & 1.1 & 2.1 & 0.50 & 0.18 & 173 & -12 \\
\hline & 5.1 & 1.0 & 0.055 & 0.023 & 74 & -5 \\
\hline & 10.1 & 0.7 & 0.0048 & 0.0080 & 53 & -6 \\
\hline & 50.3 & 0.3 & 0.00 & 0.0041 & 22 & 1 \\
\hline \multirow{4}{*}{$(10,4) \mu \mathrm{m}$} & 1.0 & 1.5 & 0.73 & 0.22 & 255 & 11 \\
\hline & 5.0 & 0.7 & 0.27 & 0.061 & 116 & 9 \\
\hline & 10.1 & 0.5 & 0.10 & 0.019 & 81 & 10 \\
\hline & 50.1 & 0.2 & 0.0029 & 0.0038 & 44 & -8 \\
\hline \multirow{4}{*}{$(10,8) \mu \mathrm{m}$} & 1.0 & 1.5 & 0.24 & 0.13 & 107 & -19 \\
\hline & 5.0 & 0.7 & 0.00 & 0.018 & 48 & -19 \\
\hline & 10.1 & 0.5 & 0.00 & 0.011 & 32 & -15 \\
\hline & 50.1 & 0.2 & 0.00 & 0.0036 & 15 & -18 \\
\hline
\end{tabular}


Table B.11: Summary of $370 \mathrm{keV}$ results for muscle.

\begin{tabular}{|c|c|c|c|c|c|c|}
\hline \multirow{2}{*}{$\left(r_{c e l l}, r_{n u c}\right)$} & \multicolumn{2}{|c|}{$D_{m, m}$} & \multicolumn{2}{|c|}{$f(z)$} & \multicolumn{2}{|c|}{$\sigma_{z} / \bar{z}$} \\
\hline & mGy & $\%$ unc. & $f(z=0)$ & RMSE & $\mathrm{MC} \times 100 \%$ & $\% \Delta_{f i t, M C}$ \\
\hline \multirow{4}{*}{$(5,2) \mu \mathrm{m}$} & 1.5 & 4.8 & 0.76 & 0.17 & 333 & 45 \\
\hline & 5.6 & 2.4 & 0.47 & 0.076 & 185 & 34 \\
\hline & 11.4 & 1.7 & 0.28 & 0.035 & 143 & 21 \\
\hline & 50.4 & 0.8 & 0.054 & 0.0052 & 81 & 1 \\
\hline \multirow{4}{*}{$(5,4) \mu \mathrm{m}$} & 1.5 & 4.8 & 0.58 & 0.18 & 201 & 24 \\
\hline & 5.6 & 2.4 & 0.18 & 0.041 & 117 & 9 \\
\hline & 11.4 & 1.7 & 0.060 & 0.014 & 77 & 15 \\
\hline & 50.4 & 0.8 & 0.0038 & 0.0037 & 42 & 0 \\
\hline \multirow{4}{*}{$(7.5,3) \mu \mathrm{m}$} & 1.5 & 3.1 & 0.59 & 0.16 & 246 & 12 \\
\hline & 5.2 & 1.7 & 0.25 & 0.056 & 131 & 14 \\
\hline & 10.4 & 1.2 & 0.11 & 0.020 & 99 & 7 \\
\hline & 50.4 & 0.5 & 0.013 & 0.0038 & 46 & 5 \\
\hline \multirow{4}{*}{$(7.5,6) \mu \mathrm{m}$} & 1.5 & 3.1 & 0.17 & 0.10 & 112 & -5 \\
\hline & 5.2 & 1.7 & 0.0077 & 0.020 & 61 & -5 \\
\hline & 10.4 & 1.2 & 0.00 & 0.010 & 42 & -4 \\
\hline & 50.4 & 0.5 & 0.00 & 0.0041 & 19 & -2 \\
\hline \multirow{4}{*}{$(10,4) \mu \mathrm{m}$} & 1.6 & 2.3 & 0.42 & 0.15 & 181 & -3 \\
\hline & 5.0 & 1.3 & 0.10 & 0.033 & 119 & -18 \\
\hline & 10.2 & 0.9 & 0.021 & 0.013 & 76 & -10 \\
\hline & 50.4 & 0.4 & 0.00 & 0.0039 & 31 & -1 \\
\hline \multirow{4}{*}{$(10,8) \mu \mathrm{m}$} & 1.6 & 2.3 & 0.031 & 0.071 & 78 & -21 \\
\hline & 5.0 & 1.3 & 0.00 & 0.019 & 41 & -17 \\
\hline & 10.2 & 0.9 & 0.00 & 0.010 & 29 & -18 \\
\hline & 50.4 & 0.4 & 0.00 & 0.0049 & 13 & -17 \\
\hline
\end{tabular}


Table B.12: Summary of ${ }^{60}$ Co results for muscle.

\begin{tabular}{|c|c|c|c|c|c|c|}
\hline \multirow{2}{*}{$\left(r_{\text {cell }}, r_{n u c}\right)$} & \multicolumn{2}{|c|}{$D_{m, m}$} & \multicolumn{2}{|c|}{$f(z)$} & \multicolumn{2}{|c|}{$\sigma_{z} / \bar{z}$} \\
\hline & mGy & $\%$ unc. & $f(z=0)$ & RMSE & $\mathrm{MC} \times 100 \%$ & $\% \Delta_{f i t, M C}$ \\
\hline \multirow{4}{*}{$(5,2) \mu \mathrm{m}$} & 2.7 & 3.0 & 0.45 & 0.077 & 378 & -28 \\
\hline & 5.5 & 2.1 & 0.27 & 0.044 & 246 & -21 \\
\hline & 10.6 & 1.5 & 0.14 & 0.022 & 161 & -13 \\
\hline & 52.0 & 0.7 & 0.0086 & 0.0047 & 59 & 6 \\
\hline \multirow{4}{*}{$(5,4) \mu \mathrm{m}$} & 2.7 & 3.0 & 0.19 & 0.077 & 121 & 20 \\
\hline & 5.5 & 2.1 & 0.056 & 0.030 & 95 & 7 \\
\hline & 10.6 & 1.5 & 0.020 & 0.014 & 65 & 13 \\
\hline & 52.0 & 0.7 & 0.0019 & 0.0043 & 32 & 3 \\
\hline \multirow{4}{*}{$(7.5,3) \mu \mathrm{m}$} & 2.7 & 2.1 & 0.25 & 0.086 & 154 & 6 \\
\hline & 5.4 & 1.5 & 0.10 & 0.036 & 114 & 2 \\
\hline & 10.6 & 1.1 & 0.029 & 0.017 & 83 & 0 \\
\hline & 51.6 & 0.5 & 0.00 & 0.0046 & 37 & 3 \\
\hline \multirow{4}{*}{$(7.5,6) \mu \mathrm{m}$} & 2.7 & 2.1 & 0.012 & 0.050 & 68 & -3 \\
\hline & 5.4 & 1.5 & 0.0010 & 0.025 & 47 & 0 \\
\hline & 10.6 & 1.1 & 0.00 & 0.010 & 33 & 1 \\
\hline & 51.6 & 0.5 & 0.00 & 0.0060 & 15 & -1 \\
\hline \multirow{4}{*}{$(10,4) \mu \mathrm{m}$} & 2.7 & 1.6 & 0.084 & 0.066 & 114 & -4 \\
\hline & 5.4 & 1.1 & 0.018 & 0.030 & 75 & 3 \\
\hline & 10.5 & 0.8 & 0.0029 & 0.015 & 53 & 5 \\
\hline & 52.1 & 0.4 & 0.00 & 0.0046 & 24 & 3 \\
\hline \multirow{4}{*}{$(10,8) \mu \mathrm{m}$} & 2.7 & 1.6 & 0.00 & 0.040 & 44 & -10 \\
\hline & 5.4 & 1.1 & 0.00 & 0.022 & 32 & -11 \\
\hline & 10.5 & 0.8 & 0.00 & 0.011 & 24 & -15 \\
\hline & 52.1 & 0.4 & 0.00 & 0.0060 & 11 & -14 \\
\hline
\end{tabular}


Table B.13: Summary of $20 \mathrm{keV}$ results for mammary gland.

\begin{tabular}{|c|c|c|c|c|c|c|}
\hline \multirow{2}{*}{$\left(r_{c e l l}, r_{n u c}\right)$} & \multicolumn{2}{|c|}{$D_{m, m}$} & \multicolumn{2}{|c|}{$f(z)$} & \multicolumn{2}{|c|}{$\sigma_{z} / \bar{z}$} \\
\hline & mGy & $\%$ unc. & $f(z=0)$ & RMSE & $\mathrm{MC} \times 100 \%$ & $\% \Delta_{f i t, M C}$ \\
\hline \multirow{4}{*}{$(5,2) \mu \mathrm{m}$} & 1.4 & 2.7 & 0.91 & 0.20 & 565 & 29 \\
\hline & 5.1 & 1.4 & 0.75 & 0.088 & 373 & 1 \\
\hline & 10.4 & 1.0 & 0.57 & 0.059 & 242 & 9 \\
\hline & 50.4 & 0.4 & 0.21 & 0.014 & 115 & 4 \\
\hline \multirow{4}{*}{$(5,4) \mu \mathrm{m}$} & 1.4 & 2.7 & 0.80 & 0.19 & 337 & 8 \\
\hline & 5.1 & 1.4 & 0.47 & 0.086 & 173 & 9 \\
\hline & 10.4 & 1.0 & 0.24 & 0.037 & 120 & 9 \\
\hline & 50.4 & 0.4 & 0.028 & 0.0034 & 54 & 11 \\
\hline \multirow{4}{*}{$(7.5,3) \mu \mathrm{m}$} & 1.3 & 1.6 & 0.82 & 0.17 & 438 & -5 \\
\hline & 5.1 & 0.8 & 0.50 & 0.082 & 188 & 14 \\
\hline & 10.3 & 0.6 & 0.28 & 0.038 & 126 & 20 \\
\hline & 50.2 & 0.3 & 0.047 & 0.0043 & 64 & 6 \\
\hline \multirow{4}{*}{$(7.5,6) \mu \mathrm{m}$} & 1.3 & 1.6 & 0.43 & 0.15 & 146 & 5 \\
\hline & 5.1 & 0.8 & 0.070 & 0.024 & 76 & 4 \\
\hline & 10.3 & 0.6 & 0.010 & 0.0057 & 53 & 4 \\
\hline & 50.2 & 0.3 & 0.00 & 0.0036 & 24 & 5 \\
\hline \multirow{4}{*}{$(10,4) \mu \mathrm{m}$} & 1.4 & 1.1 & 0.69 & 0.17 & 260 & 1 \\
\hline & 5.1 & 0.6 & 0.29 & 0.057 & 129 & 6 \\
\hline & 10.3 & 0.4 & 0.11 & 0.019 & 85 & 12 \\
\hline & 50.3 & 0.2 & 0.0078 & 0.0031 & 40 & 10 \\
\hline \multirow{4}{*}{$(10,8) \mu \mathrm{m}$} & 1.4 & 1.1 & 0.14 & 0.074 & 88 & 0 \\
\hline & 5.1 & 0.6 & 0.0020 & 0.014 & 49 & -7 \\
\hline & 10.3 & 0.4 & 0.00 & 0.0057 & 33 & -5 \\
\hline & 50.3 & 0.2 & 0.00 & 0.0045 & 15 & -6 \\
\hline
\end{tabular}


Table B.14: Summary of $30 \mathrm{keV}$ results for mammary gland.

\begin{tabular}{|c|c|c|c|c|c|c|}
\hline \multirow{2}{*}{$\left(r_{\text {cell }}, r_{\text {nuc }}\right)$} & \multicolumn{2}{|c|}{$D_{m, m}$} & \multicolumn{2}{|c|}{$f(z)$} & \multicolumn{2}{|c|}{$\sigma_{z} / \bar{z}$} \\
\hline & mGy & $\%$ unc. & $f(z=0)$ & RMSE & $\mathrm{MC} \times 100 \%$ & $\% \Delta_{f i t, M C}$ \\
\hline \multirow{4}{*}{$(5,2) \mu \mathrm{m}$} & 1.8 & 2.8 & 0.89 & 0.12 & 1091 & -45 \\
\hline & 5.1 & 1.7 & 0.70 & 0.079 & 452 & -21 \\
\hline & 10.3 & 1.2 & 0.54 & 0.053 & 299 & -16 \\
\hline & 50.3 & 0.5 & 0.17 & 0.011 & 124 & -8 \\
\hline \multirow{4}{*}{$(5,4) \mu \mathrm{m}$} & 1.8 & 2.8 & 0.71 & 0.16 & 330 & -6 \\
\hline & 5.1 & 1.7 & 0.40 & 0.078 & 166 & 10 \\
\hline & 10.3 & 1.2 & 0.21 & 0.027 & 193 & -33 \\
\hline & 50.3 & 0.5 & 0.017 & 0.0036 & 64 & -8 \\
\hline \multirow{4}{*}{$(7.5,3) \mu \mathrm{m}$} & 1.7 & 1.7 & 0.77 & 0.18 & 315 & 13 \\
\hline & 5.2 & 1.0 & 0.44 & 0.074 & 199 & 4 \\
\hline & 10.1 & 0.7 & 0.26 & 0.037 & 133 & 11 \\
\hline & 50.0 & 0.3 & 0.042 & 0.0048 & 65 & 3 \\
\hline \multirow{4}{*}{$(7.5,6) \mu \mathrm{m}$} & 1.7 & 1.7 & 0.34 & 0.15 & 140 & -2 \\
\hline & 5.2 & 1.0 & 0.053 & 0.027 & 78 & 1 \\
\hline & 10.1 & 0.7 & 0.0067 & 0.0075 & 55 & 3 \\
\hline & 50.0 & 0.3 & 0.00 & 0.0034 & 26 & 0 \\
\hline \multirow{4}{*}{$(10,4) \mu \mathrm{m}$} & 1.7 & 1.2 & 0.59 & 0.16 & 205 & 15 \\
\hline & 5.2 & 0.7 & 0.24 & 0.054 & 118 & 13 \\
\hline & 10.0 & 0.5 & 0.10 & 0.018 & 85 & 13 \\
\hline & 50.2 & 0.2 & 0.0069 & 0.0033 & 39 & 9 \\
\hline \multirow{4}{*}{$(10,8) \mu \mathrm{m}$} & 1.7 & 1.2 & 0.072 & 0.085 & 88 & -6 \\
\hline & 5.2 & 0.7 & 0.0010 & 0.013 & 52 & -10 \\
\hline & 10.0 & 0.5 & 0.00 & 0.0089 & 36 & -7 \\
\hline & 50.2 & 0.2 & 0.00 & 0.0035 & 16 & -5 \\
\hline
\end{tabular}


Table B.15: Summary of $50 \mathrm{keV}$ results for mammary gland.

\begin{tabular}{|c|c|c|c|c|c|c|}
\hline \multirow{2}{*}{$\left(r_{c e l l}, r_{n u c}\right)$} & \multicolumn{2}{|c|}{$D_{m, m}$} & \multicolumn{2}{|c|}{$f(z)$} & \multicolumn{2}{|c|}{$\sigma_{z} / \bar{z}$} \\
\hline & mGy & $\%$ unc. & $f(z=0)$ & RMSE & $\mathrm{MC} \times 100 \%$ & $\% \Delta_{f i t, M C}$ \\
\hline \multirow{4}{*}{$(5,2) \mu \mathrm{m}$} & 1.2 & 3.7 & 0.91 & 0.16 & 940 & -26 \\
\hline & 5.2 & 1.7 & 0.66 & 0.088 & 292 & 13 \\
\hline & 10.0 & 1.3 & 0.50 & 0.059 & 205 & 16 \\
\hline & 50.0 & 0.6 & 0.14 & 0.010 & 103 & 3 \\
\hline \multirow{4}{*}{$(5,4) \mu \mathrm{m}$} & 1.2 & 3.7 & 0.75 & 0.20 & 297 & 16 \\
\hline & 5.2 & 1.7 & 0.33 & 0.057 & 163 & -1 \\
\hline & 10.0 & 1.3 & 0.18 & 0.027 & 111 & 5 \\
\hline & 50.0 & 0.6 & 0.010 & 0.0037 & 47 & 11 \\
\hline \multirow{4}{*}{$(7.5,3) \mu \mathrm{m}$} & 1.2 & 2.2 & 0.79 & 0.21 & 320 & 21 \\
\hline & 5.2 & 1.0 & 0.39 & 0.070 & 162 & 15 \\
\hline & 10.2 & 0.7 & 0.23 & 0.035 & 127 & 5 \\
\hline & 50.1 & 0.3 & 0.030 & 0.0048 & 82 & -27 \\
\hline \multirow{4}{*}{$(7.5,6) \mu \mathrm{m}$} & 1.2 & 2.2 & 0.39 & 0.16 & 143 & -2 \\
\hline & 5.2 & 1.0 & 0.031 & 0.020 & 65 & 3 \\
\hline & 10.2 & 0.7 & 0.0019 & 0.0089 & 46 & 3 \\
\hline & 50.1 & 0.3 & 0.00 & 0.0040 & 21 & 3 \\
\hline \multirow{4}{*}{$(10,4) \mu \mathrm{m}$} & 1.1 & 1.5 & 0.67 & 0.21 & 239 & 4 \\
\hline & 5.2 & 0.7 & 0.20 & 0.046 & 111 & 5 \\
\hline & 10.1 & 0.5 & 0.071 & 0.014 & 78 & 8 \\
\hline & 50.0 & 0.2 & 0.0020 & 0.0038 & 37 & 2 \\
\hline \multirow{4}{*}{$(10,8) \mu \mathrm{m}$} & 1.1 & 1.5 & 0.10 & 0.11 & 90 & -10 \\
\hline & 5.2 & 0.7 & 0.0010 & 0.017 & 43 & -12 \\
\hline & 10.1 & 0.5 & 0.00 & 0.0091 & 30 & -8 \\
\hline & 50.0 & 0.2 & 0.00 & 0.0046 & 14 & -12 \\
\hline
\end{tabular}


Table B.16: Summary of $90 \mathrm{keV}$ results for mammary gland.

\begin{tabular}{|c|c|c|c|c|c|c|}
\hline \multirow{2}{*}{$\left(r_{c e l l}, r_{n u c}\right)$} & \multicolumn{2}{|c|}{$D_{m, m}$} & \multicolumn{2}{|c|}{$f(z)$} & \multicolumn{2}{|c|}{$\sigma_{z} / \bar{z}$} \\
\hline & mGy & $\%$ unc. & $f(z=0)$ & RMSE & $\mathrm{MC} \times 100 \%$ & $\% \Delta_{f i t, M C}$ \\
\hline \multirow{4}{*}{$(5,2) \mu \mathrm{m}$} & 1.1 & 3.4 & 0.93 & 0.23 & 497 & 64 \\
\hline & 5.1 & 1.5 & 0.72 & 0.11 & 278 & 35 \\
\hline & 10.0 & 1.1 & 0.56 & 0.066 & 222 & 21 \\
\hline & 50.0 & 0.5 & 0.19 & 0.013 & 115 & 5 \\
\hline \multirow{4}{*}{$(5,4) \mu \mathrm{m}$} & 1.1 & 3.4 & 0.81 & 0.23 & 307 & 26 \\
\hline & 5.1 & 1.5 & 0.40 & 0.081 & 145 & 23 \\
\hline & 10.0 & 1.1 & 0.22 & 0.034 & 107 & 19 \\
\hline & 50.0 & 0.5 & 0.027 & 0.0038 & 51 & 11 \\
\hline \multirow{4}{*}{$(7.5,3) \mu \mathrm{m}$} & 1.1 & 2.0 & 0.82 & 0.19 & 404 & 8 \\
\hline & 5.1 & 0.9 & 0.47 & 0.082 & 184 & 12 \\
\hline & 10.1 & 0.7 & 0.29 & 0.041 & 142 & 3 \\
\hline & 50.0 & 0.3 & 0.046 & 0.0050 & 66 & -1 \\
\hline \multirow{4}{*}{$(7.5,6) \mu \mathrm{m}$} & 1.1 & 2.0 & 0.47 & 0.17 & 155 & -4 \\
\hline & 5.1 & 0.9 & 0.065 & 0.022 & 74 & -5 \\
\hline & 10.1 & 0.7 & 0.012 & 0.0078 & 54 & -8 \\
\hline & 50.0 & 0.3 & 0.00 & 0.0038 & 25 & -11 \\
\hline \multirow{4}{*}{$(10,4) \mu \mathrm{m}$} & 1.1 & 1.4 & 0.74 & 0.24 & 244 & 11 \\
\hline & 5.0 & 0.7 & 0.29 & 0.065 & 125 & 2 \\
\hline & 10.0 & 0.5 & 0.13 & 0.023 & 94 & -4 \\
\hline & 50.1 & 0.2 & 0.00 & 0.0034 & 38 & 6 \\
\hline \multirow{4}{*}{$(10,8) \mu \mathrm{m}$} & 1.1 & 1.4 & 0.17 & 0.11 & 97 & -15 \\
\hline & 5.0 & 0.7 & 0.0059 & 0.011 & 47 & -18 \\
\hline & 10.0 & 0.5 & 0.00 & 0.0066 & 34 & -19 \\
\hline & 50.1 & 0.2 & 0.00 & 0.0045 & 15 & -19 \\
\hline
\end{tabular}


Table B.17: Summary of $370 \mathrm{keV}$ results for mammary gland.

\begin{tabular}{|c|c|c|c|c|c|c|}
\hline \multirow{2}{*}{$\left(r_{\text {cell }}, r_{n u c}\right)$} & \multicolumn{2}{|c|}{$D_{m, m}$} & \multicolumn{2}{|c|}{$f(z)$} & \multicolumn{2}{|c|}{$\sigma_{z} / \bar{z}$} \\
\hline & mGy & $\%$ unc. & $f(z=0)$ & RMSE & $\mathrm{MC} \times 100 \%$ & $\% \Delta_{f i t, M C}$ \\
\hline \multirow{4}{*}{$(5,2) \mu \mathrm{m}$} & 1.8 & 4.4 & 0.76 & 0.14 & 450 & -2 \\
\hline & 5.5 & 2.5 & 0.48 & 0.070 & 242 & 3 \\
\hline & 11.8 & 1.7 & 0.26 & 0.033 & 158 & 8 \\
\hline & 50.0 & 0.8 & 0.054 & 0.0054 & 76 & 8 \\
\hline \multirow{4}{*}{$(5,4) \mu \mathrm{m}$} & 1.8 & 4.4 & 0.50 & 0.14 & 195 & 16 \\
\hline & 5.5 & 2.5 & 0.17 & 0.040 & 116 & 10 \\
\hline & 11.8 & 1.7 & 0.049 & 0.012 & 76 & 15 \\
\hline & 50.0 & 0.8 & 0.0057 & 0.0042 & 41 & 4 \\
\hline \multirow{4}{*}{$(7.5,3) \mu \mathrm{m}$} & 1.6 & 3.0 & 0.55 & 0.15 & 212 & 25 \\
\hline & 5.2 & 1.7 & 0.22 & 0.045 & 145 & 3 \\
\hline & 11.6 & 1.1 & 0.083 & 0.015 & 104 & -4 \\
\hline & 50.0 & 0.5 & 0.0086 & 0.0039 & 51 & -5 \\
\hline \multirow{4}{*}{$(7.5,6) \mu \mathrm{m}$} & 1.6 & 3.0 & 0.17 & 0.11 & 114 & -11 \\
\hline & 5.2 & 1.7 & 0.0058 & 0.027 & 61 & -6 \\
\hline & 11.6 & 1.1 & 0.00 & 0.010 & 41 & -5 \\
\hline & 50.0 & 0.5 & 0.00 & 0.0051 & 20 & -5 \\
\hline \multirow{4}{*}{$(10,4) \mu \mathrm{m}$} & 1.7 & 2.2 & 0.38 & 0.14 & 151 & 11 \\
\hline & 5.4 & 1.2 & 0.071 & 0.029 & 91 & 4 \\
\hline & 11.6 & 0.8 & 0.010 & 0.010 & 62 & 5 \\
\hline & 51.3 & 0.4 & 0.00 & 0.0045 & 29 & 7 \\
\hline \multirow{4}{*}{$(10,8) \mu \mathrm{m}$} & 1.7 & 2.2 & 0.018 & 0.069 & 73 & -19 \\
\hline & 5.4 & 1.2 & 0.00 & 0.018 & 42 & -20 \\
\hline & 11.6 & 0.8 & 0.00 & 0.0078 & 29 & -21 \\
\hline & 51.3 & 0.4 & 0.00 & 0.0046 & 14 & -22 \\
\hline
\end{tabular}


Table B.18: Summary of ${ }^{60}$ Co results for mammary gland.

\begin{tabular}{|c|c|c|c|c|c|c|}
\hline \multirow{2}{*}{$\left(r_{\text {cell }}, r_{\text {nuc }}\right)$} & \multicolumn{2}{|c|}{$D_{m, m}$} & \multicolumn{2}{|c|}{$f(z)$} & \multicolumn{2}{|c|}{$\overline{\sigma_{z} / \bar{z}}$} \\
\hline & mGy & $\%$ unc. & $f(z=0)$ & RMSE & $\mathrm{MC} \times 100 \%$ & $\% \Delta_{f i t, M C}$ \\
\hline \multirow{4}{*}{$(5,2) \mu \mathrm{m}$} & 2.6 & 3.1 & 0.48 & 0.10 & 367 & -23 \\
\hline & 5.0 & 2.2 & 0.30 & 0.053 & 232 & -13 \\
\hline & 10.1 & 1.6 & 0.16 & 0.026 & 152 & -6 \\
\hline & 52.5 & 0.7 & 0.016 & 0.0047 & 66 & -6 \\
\hline \multirow{4}{*}{$(5,4) \mu \mathrm{m}$} & 2.6 & 3.1 & 0.20 & 0.084 & 196 & -24 \\
\hline & 5.0 & 2.2 & 0.073 & 0.041 & 123 & -13 \\
\hline & 10.1 & 1.6 & 0.017 & 0.019 & 76 & -1 \\
\hline & 52.5 & 0.7 & 0.00 & 0.0066 & 52 & -36 \\
\hline \multirow{4}{*}{$(7.5,3) \mu \mathrm{m}$} & 2.5 & 2.2 & 0.22 & 0.083 & 227 & -24 \\
\hline & 7.4 & 1.3 & 0.063 & 0.024 & 105 & -5 \\
\hline & 12.4 & 1.0 & 0.033 & 0.015 & 78 & -1 \\
\hline & 52.3 & 0.5 & 0.0029 & 0.0040 & 35 & 8 \\
\hline \multirow{4}{*}{$(7.5,6) \mu \mathrm{m}$} & 2.5 & 2.2 & 0.0077 & 0.063 & 74 & -6 \\
\hline & 7.4 & 1.3 & 0.00 & 0.021 & 42 & -5 \\
\hline & 12.4 & 1.0 & 0.00 & 0.011 & 32 & -2 \\
\hline & 52.3 & 0.5 & 0.00 & 0.0036 & 14 & 5 \\
\hline \multirow{4}{*}{$(10,4) \mu \mathrm{m}$} & 2.4 & 1.7 & 0.10 & 0.072 & 115 & 0 \\
\hline & 7.4 & 1.0 & 0.013 & 0.023 & 66 & 0 \\
\hline & 12.4 & 0.7 & 0.0039 & 0.015 & 51 & 0 \\
\hline & 52.1 & 0.4 & 0.00 & 0.0042 & 23 & 8 \\
\hline \multirow{4}{*}{$(10,8) \mu \mathrm{m}$} & 2.4 & 1.7 & 0.00 & 0.043 & 46 & -8 \\
\hline & 7.4 & 1.0 & 0.00 & 0.013 & 27 & -11 \\
\hline & 12.4 & 0.7 & 0.00 & 0.010 & 21 & -12 \\
\hline & 52.1 & 0.4 & 0.00 & 0.0052 & 10 & -13 \\
\hline
\end{tabular}




\section{References}

1 W. Parker and H. Patrocinio, Clinical treatment planning in external photon beam radiotherapy, in Radiation Oncology Physics: A Handbook for Teachers and Students, edited by E. B. Podgorsak, pages 219-272, International Atomic Energy Agency, Vienna, Austria, 2005.

2 L. J. Verhey, Comparison of three-dimensional conformal radiation therapy and intensity-modulated radiation therapy systems, Semin. Radiat. Oncol. 9, 78-98 (1999).

3 K. Otto, Volumetric modulated arc therapy: IMRT in a single gantry arc, Med. Phys. 35, 310 - 317 (2008).

4 C. Ozhasoglu et al., Synchrony - Cyberknife respiratory compensation technology, Med. Dosim. 33, 117 - 123 (2008).

5 S. F. Kry, M. Salehpour, D. S. Followill, M. Stovall, D. A. Kuban, R. A. White, and I. I. Rosen, The calculated risk of fatal secondary malignancies from intensitymodulated radiation therapy, Int. J. Radiat. Oncol. Biol. Phys. 62, 1195 - 1203 (2005).

6 P. Mayles, Total body irradiation, in Handbook of Radiotherapy Physics, edited by P. Mayles, A. Nahum, and J.-P. Rosenwald, pages 1033 - 1041, Taylor and Francis, New York, 2007.

7 J. Y. C. Wong, J. Rosenthal, A. Liu, T. Schultheiss, S. Forman, and G. Somlo, Image-guided total-marrow irradiation using helical tomotherapy in patients with multiple myeloma and acute leukemia undergoing hematopoietic cell transplantation, Int. J. Radiat. Oncol. Biol. Phys. 73, 273 - 279 (2009).

8 F. M. Khan and J. P. Gibbons, The Physics of Radiation Therapy, Lipincott Williams and Wilkins, Philadelphia, PA, 5th edition, 2014.

9 D. R. Dance, S. Christofides, A. D. A. Maidment, I. D. McLean, and K. H. Ng, editors, Diagnostic Radiology Physics: A Handbook for Teachers and Students, International Atomic Energy Agency, Vienna, Austria, 2014.

10 E. J. Hall and A. J. Giaccia, Radiobiology for the radiologist, 7th ed., Lippincott Williams \& Wilkins, Philadelphia, 2012. 
11 E. Hall and D. Brenner, Cancer risks from diagnostic radiology, Brit. J. Radiol. 81, 362-378 (2008).

12 Cancer Care Ontario, Ontario Breast Screening Program 2011 Report, Technical report, Toronto, Canada. https://www.cancercareontario.ca/en/cancer-careontario/programs/screening-programs/ontario-breast-obsp/report, 2013.

M. J. Yaffe and A. D. A. Maidment, Mammography, in Diagnostic Radiology Physics: A Handbook for Teachers and Students, edited by D. R. Dance, S. Christofides, A. D. A. Maidment, I. D. McLean, and K. H. Ng, pages 209-239, International Atomic Energy Agency, Vienna, Austria, 2014.

14 S. Webb, Conformal and intensity-modulated radiotherapy, in Handbook of Radiotherapy Physics, edited by P. Mayles, A. Nahum, and J.-P. Rosenwald, pages 943 - 973, Taylor and Francis, New York, 2007.

15 ICRU, Report 85: Fundamental Quantities and Units for Ionizing Radiation, J of the ICRU 11, 1 - 33 (2011).

16 H. H. Rossi and M. Zaider, Microdosimetry and its applications, Springer, Berlin, 1996.

17 H. H. Rossi and M. Zaider, Elements of microdosimetry, Med. Phys. 18, 1085-1092 (1991).

18 A. Brahme, Dosimetric Precision Requirements in Radiation Therapy, Acta Radiol. Oncol. 23, 379-391 (1984).

19 L. Lindborg and A. Brahme, Influence of microdosimetric quantities on observed dose-response relationships in radiation therapy, Radiat. Res. 124, S23-S28 (1990).

S. P. Verma and N. Sonwalkar, Structural Changes in Plasma Membranes Prepared from Irradiated Chinese Hamster V79 Cells as Revealed by Raman Spectroscopy, Radiat. Res. 126, 27 - 35 (1991).

21 R. J. Lakshmi, V. B. Kartha, C. M. Krishna, J. G. R. Solomon, G. Ullas, and P. U. Devi, Tissue Raman Spectroscopy for the Study of Radiation Damage: Brain Irradiation of Mice, Radiat. Res. 157, 175 - 182 (2002).

22 H. Nagasawa and J. B. Little, Induction of sister chromatid exchanges by extremely low doses of $\alpha$-particles, Cancer Res. 52, $6394-6396$ (1992).

23 L. A. DeWerd, S. D. Davis, L. J. Bartol, and F. Grenzow, Ionization chamber instrumentation, in Clinical dosimetry measurements in radiotherapy, edited by D. W. O. Rogers and J. E. Cygler, pages 181 - 204, Medical Physics Publishing, Madison, Wisconsin, 2009.

24 J. Izewska and G. Rajan, Radiation dosimeters, in Radiation Oncology Physics: A Handbook for Teachers and Students, edited by E. B. Podgorsak, pages 71-99, International Atomic Energy Agency, Vienna, Austria, 2005. 
D. L. Zellmer, M. T. Gillin, and J. F. Wilson, Microdosimetric single event spectra of Ytterbium-169 compared with commonly used brachytherapy sources and teletherapy beams, Int. J. Radiat. Oncol. Biol. Phys. 23, 627 - 632 (1992).

G. Schettino, S. T. Al-Rashid, and K. M. Prise, Radiation microbeams as spatial and temporal probes of subcellular and tissue response, Mutat. Res. 704, 68 - 77 (2010).

S. Rahmanian, M. Niklas, A. Abdollahi, O. Jäkel, and S. Greilich, Application of fluorescent nuclear track detectors for cellular dosimetry, Phys. Med. Biol. 62, $2719-2740$ (2017).

I. Kawrakow, E. Mainegra-Hing, D. W. O. Rogers, F. Tessier, and B. R. B. Walters, The EGSnrc Code System: Monte Carlo simulation of electron and photon transport, NRC Technical Report PIRS-701 v4-2-3-2, National Research Council Canada, Ottawa, Canada. http://nrc-cnrc.github.io/EGSnrc/doc/pirs701egsnrc.pdf, 2011.

I. Kawrakow, Accurate condensed history Monte Carlo simulation of electron transport. I. EGSnrc, the new EGS4 version, Med. Phys. 27, 485 - 498 (2000).

D. W. O. Rogers and A. F. Bielajew, Monte Carlo techniques of electron and photon transport for radiation dosimetry, in The Dosimetry of Ionizing Radiation, Vol III, edited by K. R. Kase, B. E. Bjärngard, and F. H. Attix, pages 427 539, Academic Press, 1990.

31 I. J. Chetty et al., Report of the AAPM Task Group No. 105: Issues associated with clinical implementation of Monte Carlo-based photon and electron external beam treatment planning, Med. Phys. 34, 4818 -4853 (2007).

L. Beaulieu et al., Report of the Task Group 186 on model-based dose calculation methods in brachytherapy beyond the TG-43 formalism: Current status and recommendations for clinical implementation, Med. Phys. 39, 6208 - 6236 (2012).

I. Sechopoulos et al., Monte Carlo reference data sets for imaging research: Executive summary of the report of AAPM Research Committee Task Group 195, Med. Phys. 42, 5679-5691 (2015).

S. Lehnert, Biomolecular action of ionizing radiation, Taylor \& Francis, London, 2008.

R. M. Thomson, A. Carlsson Tedgren, and J. F. Williamson, On the biological basis for competing macroscopic dose descriptors for kilovoltage dosimetry: cellular dosimetry for brachytherapy and diagnostic radiology, Phys. Med. Biol. 58, 1123 -1150 (2013).

36 A. M. Kuzin, On the role of DNA in the radiation damage of the cell, Int. J. Radiat. Biol. Relat. Stud. Phys. Chem. Med. 6, 201-209 (1963).

D. T. Goodhead, Energy deposition stochastics and track structure: what about the target?, Radiat. Prot. Dosim. 122, 3 - 15 (2006). 
L. Lindborg, M. Hultqvist, A. C. Tedgren, and H. Nikjoo, Lineal energy and radiation quality in radiation therapy: model calculations and comparison with experiment, Phys. Med. Biol. 58, 3089-3105 (2013).

K. M. Prise, G. Schettino, M. Folkard, and K. D. Held, New insights on cell death from radiation exposure, Lancet Oncol. 6, 520 - 528 (2005).

C. Shao, M. Folkard, B. D. Michael, and K. M. Prise, Targeted cytoplasmic irradiation induces bystander responses, Proc. Natl. Acad. Sci. U.S.A. 101, 13495 - 13500 (2004).

Z. Kuncic, H. L. Byrne, A. L. McNamara, S. Guatelli, W. Domanova, and S. Incerti, In silico nanodosimetry: new insights into nontargeted biological responses to radiation, Comput. Math. Methods. Med. 2012, 147252 (2012).

D. R. Green and J. C. Reed, Mitochondria and apoptosis, Science 281, 1309 (1998).

T. Yamamori, H. Yasui, M. Yamazumi, Y. Wada, Y. Nakamura, H. Nakamura, and O. Inanami, Ionizing radiation induces mitochondrial reactive oxygen species production accompanied by upregulation of mitochondrial electron transport chain function and mitochondrial content under control of the cell cycle checkpoint, Free Radic. Biol. Med. 53, 260-270 (2012).

W. W.-Y. Kam and R. B. Banati, Effects of ionizing radiation on mitochondria, Free Radic. Biol. Med. 65, 607-619 (2013).

M. J. Rivard et al., Update of AAPM Task Group No. 43 Report: A revised AAPM protocol for brachytherapy dose calculations, Med. Phys. 31, 633 - 674 (2004).

A. E. Nahum, Cavity theory, stopping power ratios, correction factors, in Clinical Dosimetry Measurements in Radiotherapy, edited by D. W. O. Rogers and J. E. Cygler, Medical Physics Publishing, Madison, Wisconsin, 2009.

7 J. V. Siebers, P. J. Keall, A. E. Nahum, and R. Mohan, Converting absorbed dose to medium to absorbed dose to water for Monte Carlo based photon beam dose calculation, Phys. Med. Biol. 45, 983 - 995 (2000).

P. R. Almond, P. J. Biggs, B. M. Coursey, W. F. Hanson, M. S. Huq, R. Nath, and D. W. O. Rogers, AAPM's TG-51 protocol for clinical reference dosimetry of high-energy photon and electron beams, Med. Phys. 26, 1847 - 1870 (1999).

H. H. Liu and P. Keall, Point/Counterpoint: $\mathrm{D}_{m}$ rather than $\mathrm{D}_{w}$ should be used in Monte Carlo treatment planning, Med. Phys. 29, 922 - 924 (2002).

B. R. B. Walters, R. Kramer, and I. Kawrakow, Dose to medium versus dose to water as an estimator of dose to sensitive skeletal tissue, Phys. Med. Biol. 55, 4535 -4546 (2010).

P. Andreo, Dose to 'water-like' media or dose to tissue in MV photons radiotherapy treatment planning: still a matter of debate, Phys. Med. Biol. 60, 309 - 337 (2015). 

R. M. Thomson, F. Verhaegen, T. A. Wareing, and J. F. Williamson, Report of the Task Group 186 on model-based dose calculation methods in brachytherapy beyond the TG-43 formalism: Current status and recommendations for clinical implementation, Med. Phys. 39, 6208 - 6236 (2012).

53

S. Incerti, H. Seznec, M. Simon, P. Barberet, C. Habchi, and P. Moretto, Monte Carlo dosimetry for targeted irradiation of individual cells using a microbeam facility, Radiat. Prot. Dosim. 133, 1 - 11 (2009).

54

P. Barberet et al., Monte-Carlo dosimetry on a realistic cell monolayer geometry exposed to alpha particles, Phys. Med. Biol. 57, 2189 - 2207 (2012).

S. A. Enger, A. Ahnesjo, F. Verhaegen, and L. Beaulieu, Dose to tissue medium or water cavities as surrogate for the dose to cell nuclei at brachytherapy photon energies, Phys. Med. Biol. 57, 4489 - 4500 (2012).

H. L. Byrne, A. L. McNamara, W. Domanova, S. Guatelli, and Z. Kuncic, Radiation damage on sub-cellular scales: beyond DNA, Phys. Med. Biol. 58, 1251 1267 (2013).

M. Douglass, E. Bezak, and S. Penfold, Development of a randomized 3D cell model for Monte Carlo microdosimetry simulations, Med. Phys. 39, 3509 - 3519 (2012). Implications on clinical scenario of gold nanoparticle radiosensitization in regards to photon energy, nanoparticle size, concentration and location, Phys. Med. Biol. 56, $4631-4647$ (2011).

60 Z. Cai, J.-P. Pignol, N. Chattopadhyay, Y. L. Kwon, E. Lechtman, and R. M. Reilly, Investigation of the effects of cell model and subcellular location of gold nanoparticles on nuclear dose enhancement factors using Monte Carlo simulation, Med. Phys. 40, 114101 (2013).

61 M. Douglass, E. Bezak, and S. Penfold, Monte Carlo investigation of the increased radiation deposition due to gold nanoparticles using kilovoltage and megavoltage photons in a 3D randomized cell model, Med. Phys. 40, 071710 (2013).

B. Koger and C. Kirkby, A method for converting dose-to-medium to dose-totissue in Monte Carlo studies of gold nanoparticle-enhanced radiotherapy, Phys. Med. Biol. 61, 2014 - 2024 (2016).

$63 \AA$. Carlsson Tedgren and G. Alm Carlsson, Specification of absorbed dose to water using model-based dose calculation algorithms for treatment planning in brachytherapy, Phys. Med. Biol. 58, 2561 - 2579 (2013). 
F. Villegas, N. Tilly, and A. Ahnesjö, Monte Carlo calculated microdosimetric spread for cell nucleus-sized targets exposed to brachytherapy ${ }^{125} \mathrm{I}$ and ${ }^{192} \mathrm{Ir}$ sources and ${ }^{60}$ Co cell irradiation, Phys. Med. Biol. 58, 6149 - 6162 (2013).

F. Villegas and A. Ahnesjö, Reply to the comment on 'Monte Carlo calculated microdosimetric spread for cell nucleus-sized targets exposed to brachytherapy ${ }^{125} \mathrm{I}$ and ${ }^{192}$ Ir sources and ${ }^{60}$ Co cell irradiation', Phys. Med. Biol. 61, $5103-5106$ (2016).

M. Cunha, E. Testa, O. V. Komova, E. A. Nasonova, L. A. Mel'nikova, N. L. Shmakova, and M. Beuve, Modeling cell response to low doses of photon irradiation - Part 1: on the origin of fluctuations, Radiat. Environ. Biophys. 55, 19-30 (2016).

C. M. Poole, A. Ahnesjö, and S. A. Enger, Determination of subcellular compartment sizes for estimating dose variations in radiotherapy, Radiat. Prot. Dosim. 166, $361-364$ (2015).

S. Standring and H. Gray, Gray's anatomy: The anatomical basis of clinical practice, 40th ed., Churchill Livingstone/Elsevier, Edinburgh, 2008.

A. Maguire, I. Vegacarrascal, L. White, B. McClean, O. Howe, F. M. Lyng, and A. D. Meade, Analyses of ionizing radiation effects in vitro in peripheral blood lymphocytes with Raman spectroscopy, Radiat. Res. 183, 407 - 416 (2015).

C. H. Allen, A. Kumar, S. Qutob, B. Nyiri, V. Chauhan, and S. Murugkar, Raman micro-spectroscopy analysis of human lens epithelial cells exposed to a low-doserange of ionizing radiation, Phys. Med. Biol. 63, 025002 (2018).

B. R. B. Walters, I. Kawrakow, and D. W. O. Rogers, History by history statistical estimators in the BEAM code system, Med. Phys. 29, 2745 - 2752 (2002).

J. Wulff, K. Zink, and I. Kawrakow, Efficiency improvements for ion chamber calculations in high energy photon beams, Med. Phys. 35, 1328- 1336 (2008).

I. Kawrakow, E. Mainegra-Hing, F. Tessier, and B. R. B. Walters, The EGSnrc C++ class library, Technical Report PIRS-898 (rev A), National Research Council Canada, Ottawa, Canada. http://nrccnrc.github.io/EGSnrc/doc/pirs898/index.html, 2009.

74 D. W. O. Rogers, I. Kawrakow, J. P. Seuntjens, B. R. B. Walters, and E. Mainegra-Hing, NRC user codes for EGSnrc, NRC Technical Report PIRS-702 (rev C), National Research Council Canada, Ottawa, Canada. http://www.irs.inms.nrc.ca/EGSnrc/PIRS702/, 2011.

75 ICRU, Stopping powers for electrons and positrons, ICRU Report 37, ICRU, Bethesda, MD., 1984.

M. J. Berger, ESTAR, PSTAR and ASTAR: Computer Programs for Calculating Stopping-Power and Ranges for Electrons, Protons, and Helium Ions, NIST Report NISTIR-4999 (Washington DC), available on-line at http://physics.nist.gov/Star (1992). 
77

78

79

81

84

85

90

86 R. L. Maughan, P. J. Chuba, A. T. Porter, E. Ben-Josef, and D. R. Lucas, The

M. J. Berger et al., XCOM: Photon cross section database (version 1.5), Technical report, NIST, Gaithersburg, MD, http://physics.nist.gov/xcom, 2010.

J. H. Hubbell, Review of photon interaction cross section data in the medical and biological context, Phys. Med. Biol. 44, R1 - R22 (1999).

D. E. Cullen, J. H. Hubbell, and L. Kissel, EDPL97: the Evaluated Photon Data Library, '97 Version, Report UCRL-50400, Vol 6, Rev 5, LLNL, Livermore, Ca, 1997.

S. M. Seltzer and M. J. Berger, Bremsstrahlung energy spectra from electrons with kinetic energy from $1 \mathrm{keV}$ to $10 \mathrm{GeV}$ incident on screened nuclei and orbital electrons of neutral atoms with $\mathrm{Z}=1-100$, Atomic Data and Nuclear Data Tables 35, $345-418$ (1986).

M. J. P. Chamberland, R. E. P. Taylor, D. W. O. Rogers, and R. M. Thomson, egs_brachy: a versatile and fast Monte Carlo code for brachytherapy, Phys. Med. Biol. 61, 8214 - 8231 (2016).

R. M. Thomson, R. E. P. Taylor, M. J. P. Chamberland, and D. W. O. Rogers, User Manual for egs_brachy, a versatile and fast EGSnrc application for brachytherapy, Technical report, Carleton Laboratory for Radiotherapy Physics, Ottawa, Canada. https://clrp-code.github.io/egs_brachy/egs_brachy_user_manual.pdf, 2017.

83 H. E. Johns and J. R. Cunningham, The physics of radiology, 4th ed., Charles C. Thomas, Springfield, Illinois, 1983.

H. Q. Woodard and D. R. White, The composition of body tissues, Brit. J. Radiol. 59, 1209 - 1219 (1986).

ICRU, Tissue Substitutes in Radiation Dosimetry and Measurements, ICRU Report 44, ICRU, Washington D.C., 1989. elemental composition of tumors: Kerma data for neutrons, Med. Phys. 24, 1241 - 1244 (1997).

87 C. Terryn, J. Michel, X. Thomas, D. Laurent-Maquin, and G. Balossier, Implementation of subcellular water mapping by electron energy loss spectroscopy in a medium-voltage scanning transmission electron microscope, Eur. Biophys. J. 33, $321-327$ (2004).

S. Bolkent and K. Zierold, Effects of the ionophores valinomycin, ionomycin and gramicidin A on the element compartmentation in cultured rat hepatocytes, Toxicol. In Vitro 16, 159 - 165 (2002).

89 J. E. Downing, W. M. Christopherson, and W. L. Broghamer, Nuclear water content during carcinogenesis, Cancer 15, 1176 - 1180 (1962).

T. Yamaguchi, C. Muraiso, I. Furuno-Fukushi, and A. Tsuboi, Water content in cultured mammalian cells for dosimetry of beta-rays from tritiated water, J. Radiat. Res. 31, 333 - 339 (1990). 
91 A. J. Saubermann and V. L. Scheid, Elemental composition and water content of neuron and glial cells in the central nervous system of the North American medicinal leech (Macrobdella decora), J. Neurochem. 44, 825 - 834 (1985).

I. L. Cameron, N. K. R. Smith, T. B. Pool, and R. L. Sparks, Intracellular concentration of sodium and other elements as related to mitogenesis and oncogenesis invivo, Cancer Res. 40, 1493 - 1500 (1980).

K. Zierold, J. Michel, C. Terryn, and G. Balossier, The distribution of light elements in biological cells measured by electron probe X-ray microanalysis of cryosections, Microsc. Microanal. 11, 138 - 145 (2005).

ICRP, Basic Anatomical and Physiological Data for Use in Radiological Protection: Reference Values, Annals of the ICRP 89, ICRP, Washington D.C., 2003.

P. H. Tan, B. B. Goh, G. Chiang, and B. H. Bay, Correlation of nuclear morphometry with pathologic parameters in ductal carcinoma in situ of the breast, Mod. Pathol. 14, 937-941 (2001).

L. G. Koss and M. R. Melamed, Koss' diagnostic cytology and its histopathologic bases, 5th ed., Lippincott Williams \& Wilkins, Philadelphia, 2006.

S. Watanabe, K. Okita, T. Harada, T. Kodama, Y. Numa, T. Takemoto, and T. Takahashi, Morphologic studies of the liver cell dysplasia, Cancer 51, 21972205 (1983).

B. J. Bain, Blood cells: A practical guide, 4th ed., Blackwell Publishing, Malden, Massachusetts, USA, 2006.

S. Park, R. R. Ang, S. P. Duffy, J. Bazov, K. N. Chi, P. C. Black, and H. Ma, Morphological differences between circulating tumor cells from prostate cancer patients and cultured prostate cancer cells, PLoS ONE 9, e85264 (2014).

100 A.-C. Wéra, L. Barazzuol, J. C. G. Jeynes, M. J. Merchant, M. Suzuki, and K. J. Kirkby, Influence of the nucleus area distribution on the survival fraction after charged particles broad beam irradiation, Phys. Med. Biol. 59, 4197-4211 (2014).

101 C.-P. Adler, H. Friedburg, G. W. Herget, M. Neuburger, and H. Schwalb, Variability of cardiomyocyte DNA content, ploidy level and nuclear number in mammalian hearts, Virchows Arch. 429, 159-164 (1996).

102 Z. L. Cai, J. P. Pignol, C. Chan, and R. M. Reilly, Cellular Dosimetry of In111 Using Monte Carlo N-Particle Computer Code: Comparison with Analytic Methods and Correlation with In Vitro Cytotoxicity, J. of Nucl. Med. 51, 462 $470(2010)$.

103 T. K. Lee, R. D. Horner, J. F. Silverman, Y. H. Chen, C. Jenny, and C. W. Scarantino, Morphometric and morphologic evaluations in stage-III non-small cell lung cancers - Prognostic significance of quantitative assessment of infiltrating lymphoid cells, Cancer 63, 309 - 316 (1989). 
V. Backman et al., Detection of preinvasive cancer cells, Nature 406, 35-36 (2000).

K. J. Pienta and D. S. Coffey, Correlation of nuclear morphometry with progression of breast cancer, Cancer 68, 2012-2016 (1991).

T. K. Lee, J. F. Silverman, R. D. Horner, and C. W. Scarantino, Overlap of nuclear diameters in lung cancer cells, Anal. Quant. Cytol. Histol. 12, 275-278 (1990).

R. Nafe, K. Franz, W. Schlote, and B. Schneider, Morphology of tumor cell nuclei is significantly related with survival time of patients with glioblastomas, Clin. Cancer Res. 11, 2141-2148 (2005).

M. Ladekarl and F. B. Sorensen, Quantitative histopathological variables in in-situ and invasive ductal and lobular carcinomas of the breast, APMIS 101, 895 - 903 (1993).

M. L. Bonnelykke-Behrndtz, F. B. Sorensen, and T. E. Damsgaard, Stereological quantification of tumor volume, mean nuclear volume and total number of melanoma cells correlated with morbidity and mortality, APMSIS 116, 903 - 911 (2008).

R. K. Jain, Transport of molecules in the tumor interstitium: A review, Cancer Res. 47, 3039 - 3051 (1987).

L. M. M. F. Demarchi et al., Prognostic values of stromal proportion and PCNA, Ki-67, and p53 proteins in patients with resected adenocarcinoma of the lung, Mod. Pathol. 13, 511 - 520 (2000).

L. T. Perelman et al., Observation of periodic fine structure in reflectance from biological tissue: a new technique for measuring nuclear size distribution, Phys. Rev. Lett. 80, 627-630 (1998).

C. N. Battlehner, P. H. N. Saldiva, C. R. R. Carvalho, T. Y. Takagaki, G. S. Montes, R. N. Younes, and V. L. Capelozzi, Nuclear/cytoplasmic ratio correlates strongly with survival in non-disseminated neuroendocrine carcinoma of the lung, Histopathology 22, 31-34 (1993).

W. H. Wolberg, W. N. Street, and O. L. Mangasarian, Importance of nuclear morphology in breast cancer prognosis, Clin. Cancer. Res. 5, 3542-3548 (1999).

A. El-Hussein, A. K. Kassem, H. Ismail, and M. A. Harith, Exploiting LIBS as a spectrochemical analytical technique in diagnosis of some types of human malignancies, Talanta 82, 495 - 501 (2010).

H. L. Byrne, W. Domanova, A. L. McNamara, S. Incerti, and Z. Kuncic, The cytoplasm as a radiation target: an in silico study of microbeam cell irradiation, Phys. Med. Biol. 60, 2325 - 2337 (2015).

D. R. White, H. Q. Woodard, and S. M. Hammond, Average soft-tissue and bone models for use in radiation dosimetry, Brit. J. Radiol. 60, 907 - 913 (1987). 
T. A. Carrillo-Cázares and E. Torres-García, Monte Carlo mitonchondrial dosimetry and microdosimetry of ${ }^{131} \mathrm{I}$, Radiat. Prot. Dosim. 153, 411 - 416 (2013).

M. Šefl, S. Incerti, G. Papamichael, and D. Emfietzoglou, Calculation of cellular $S$-values using Geant4-DNA: The effect of cell geometry, Appl. Radiat. Isotopes 104, 113 - 123 (2015).

T. P. Selvam and D. W. O. Rogers, Inclusion of Bragg-Gray theory stopping-power ratios in the SPRRZnrc code, Technical Report CLRP 06-01, Carleton Laboratory for Radiotherapy Physics, Carleton University, Ottawa K1S 5B6, Canada, 2006.

L. V. Spencer and F. H. Attix, A theory of cavity ionization, Radiat. Res. 3, 239 -254 (1955).

A. M. Kellerer, Considerations on the random traversal of convex bodies and solutions for general cylinders, Radiat. Res. 47, 359 - 376 (1971).

T. E. Burlin, A general theory of cavity ionization, Br. J. Radiol. 39, 727 - 734 (1966).

T. E. Burlin and F. K. Chan, The Effect of the Wall on the Fricke Dosimeter, Int'l J App Rad Isotop 20, 767 - 775 (1969).

A. Janssens, G. Eggermont, R. Jacobs, and G. Thielens, Spectrum perturbation and energy deposition models for stopping power ratio calculations in general cavity theory, Phys. Med. Biol. 19, 619 - 627 (1974).

R. Loevinger, The dosimetry of beta sources in tissue: the point-source function, Radiology 66, 55-62 (1956).

P. N. Mobit, P. Mayles, and A. E. Nahum, The quality dependence of LiF TLD in megavoltage photon beams: Monte Carlo simulation and experiments, Phys. Med. Biol. 41, 387 - 398 (1996).

N. S. Patel, S.-T. Chiu-Tsao, J. F. Williamson, P. Fan, T. Duckworth, D. Shasha, and L. B. Harrison, Thermoluminescent dosimetry of the Symmetra ${ }^{125}$ I model I25.S06 interstitial brachytherapy seed, Med. Phys. 28, 1761 - 1769 (2001).

L. Antonovic, H. Gustafsson, G. Alm Carlsson, and A. Carlsson Tedgren, Evaluation of a lithium formate EPR dosimetry system for dose measurements around ${ }^{192}$ Ir brachytherapy sources, Med. Phys. 36, 2236-2247 (2009).

S. Kumar, D. D. Deshpande, and A. E. Nahum, Dosimetric response of variablesize cavities in photon-irradiated media and the behaviour of the Spencer-Attix cavity integral with increasing $\Delta$, Phys. Med. Biol. 61, 2680 - 2704 (2016).

P. R. Almond and H. Svensson, Ionization chamber dosimetry for photon and electron Beams, Acta Radiol. Ther. Phys. Biol. 16, 177 - 186 (1977).

L. A. Buckley, I. Kawrakow, and D. W. O. Rogers, An EGSnrc investigation of cavity theory for ion chambers measuring air kerma, Med. Phys. 30, 1211 - 1218 (2003). 
H. Nikjoo, S. Uehara, D. Emfietzoglou, and F. A. Cucinotta, Track-structure codes in radiation research, Radiat. Meas. 41, 1052 - 1074 (2006).

S. Plimpton, Fast Parallel Algorithms for Short-Range Molecular Dynamics, J. Comp. Phys. 117, 1-19, see http://lammps.sandia.gov (1995).

D. W. O. Rogers, G. M. Ewart, A. F. Bielajew, and G. van Dyk, Calculation of Electron Contamination in a ${ }^{60} \mathrm{Co}$ Therapy Beam, in "Proceedings of the IAEA International Symposium on Dosimetry in Radiotherapy" (IAEA, Vienna), Vol 1 , $303-312$ (1988).

ICRU, Microdosimetry, ICRU Report 36, ICRU, Bethesda, MD., 1993.

S. S. Shapiro and M. B. Wilk, An analysis of variance test for normality (complete samples), Biometrika 52, 591-611 (1965).

A. Ghasemi and S. Zahediasl, Normality tests for statistical analysis: a guide for non-statisticians, Int. J. Endocrinol. Metab. 10, 486 - 489 (2012).

S. Devpura et al., Vision 20/20: The role of Raman spectroscopy in early stage cancer detection and feasibility for application in radiation therapy response assessment, Med. Phys. 41, 050901 (2014).

S. J. Harder et al., Raman spectroscopy identifies radiation response in human non-small cell lung cancer xenografts, Sci. Rep. 6, 21006 (2016).

M. S. Vidyasagar, K. Maheedhar, B. M. Vadhiraja, D. J. Fernendes, V. B. Kartha, and C. M. Krishna, Prediction of radiotherapy response in cervix cancer by Raman Spectroscopy: a pilot study, Biopolymers 89, 530 - 537 (2008).

I. Delfino et al., Visible micro-Raman spectroscopy of single human mammary epithelial cells exposed to x-ray radiation, J. Biomed. Opt. 20, 035003 (2015).

M. Yasser, R. Shaikh, M. K. Chilakapati, and T. Teni, Raman Spectroscopic Study of Radioresistant Oral Cancer Sublines Established by Fractionated Ionizing Radiation, PLOS ONE 9, e97777 (2014).

Q. Matthews, A. G. Brolo, J. Lum, X. Duan, and A. Jirasek, Raman spectroscopy of single human tumour cells exposed to ionizing radiation in vitro, Phys. Med. Biol. 56, $19-38$ (2011).

Q. Matthews, A. Jirasek, J. Lum, and A. G. Brolo, Biochemical signatures of in vitro radiation response in human lung, breast and prostate tumour cells observed with Raman spectroscopy, Phys. Med. Biol. 56, 6839 - 6855 (2011).

A. D. Meade, O. Howe, V. Unterreiner, G. D. Sockalingum, H. J. Byrne, and F. M. Lyng, Vibrational spectroscopy in sensing radiobiological effects: analyses of targeted and non-targeted effects in human keratinocytes, Farad. Discuss. 187, $213-234$ (2016). 
E. Zastrow, S. K. Davis, M. Lazebnik, F. Kelcz, B. D. V. Veen, and S. C. Hagness,
Development of Anatomically Realistic Numerical Breast Phantoms With Accurate
Dielectric Properties for Modeling Microwave Interactions With the Human Breast,
IEEE Transactions on Biomedical Engineering 55,2792 - $2800(2010)$.

E. Zastrow, S. K. Davis, M. Lazebnik, F. Kelcz, B. D. V. Veen, and S. C. Hagness,
Development of Anatomically Realistic Numerical Breast Phantoms With Accurate
Dielectric Properties for Modeling Microwave Interactions With the Human Breast,
IEEE Transactions on Biomedical Enoineering 55,2792 - $2800(2010)$.

E. Zastrow, S. K. Davis, M. Lazebnik, F. Kelcz, B. D. V. Veen, and S. C. Hagness,
Development of Anatomically Realistic Numerical Breast Phantoms With Accurate
Dielectric Properties for Modeling Microwave Interactions With the Human Breast,
IEEE Transactions on Biomedical Enoineering 55,2792 - $2800(2010)$.

E. Zastrow, S. K. Davis, M. Lazebnik, F. Kelcz, B. D. V. Veen, and S. C. Hagness,
Development of Anatomically Realistic Numerical Breast Phantoms With Accurate
Dielectric Properties for Modeling Microwave Interactions With the Human Breast,
IEEE Transactions on Biomedical Engineering 55, 2792 - 2800 (2010).

J. M. Boone and J. A. Seibert, An accurate method for computer-generating tungsten anode x-ray spectra from 30 to 140 kV, Med. Phys. 24, 1661 - 1670 (1997).

R. Mohan, C. Chui, and L. Lidofsky, Energy and angular distributions of photons from medical linear accelerators, Med. Phys. 12, 592 - 597 (1985).

D. R. Dance and I. Sechopoulos, Dosimetry in x-ray-based breast imaging, Phys. Med. Biol. 61, R271 - R304 (2016).

D. R. Dance, Monte Carlo calculation of conversion factors for the estimation of mean glandular dose, Phys. Med. Biol. 35, 1211-1219 (1990).

J. M. Boone, Glandular Breast Dose for Monoenergetic and High-Energy X-ray Beams: Monte Carlo Assessment, Radiology 213, 23 - 37 (1999).

D. R. Dance, C. L. Skinner, K. C. Young, J. R. Beckett, and C. J. Kotre, Additional factors for the estimation of mean glandular breast dose using the UK mammography dosimetry protocol, Phys. Med. Biol. 45, 3225-3240 (2000).

K. C. Young and J. M. Oduko, Radiation doses received in the United Kingdom breast screening programme in 2010 to 2012, Brit. J. Radiol. 89, 20150831 (2016).

I. Sechopoulos, K. Bliznakova, X. Qin, B. Fei, and S. S. J. Feng, Characterization of the homogeneous tissue mixture approximation in breast imaging dosimetry, Med. Phys. 39, 5050-5059 (2012).

A. M. Hernandez, J. A. Seibert, and J. M. Boone, Breast dose in mammography is about $30 \%$ lower when realistic heterogeneous glandular distributions are considered, Med. Phys. 42, 6337-6348 (2015).

W. Wang, R. Qiu, L. Ren, H. Liu, Z. Wu, C. Li, Y. Niu, and J. Li, Monte Carlo calculation of conversion coefficients for dose estimation in mammography based on a 3D detailed breast model, Med. Phys. 44, 2503-2514 (2017).

C. Zyganitidis, K. Bliznakova, and N. Pallikarakis, A novel simulation algorithm for soft tissue compression, Med. Biol. Eng. Comput. 45, 661-669 (2007).

C. Hoeschen, U. Fill, M. Zankl, W. Panzer, D. Regulla, and W. Döhring, A highresolution voxel phantom of the breast for dose calculations in mammography, Radiat. Prot. Dosim. 114, 406-409 (2005).

A. Sarno, G. Mettivier, F. Di Lillo, and P. Russo, A Monte Carlo study of monoenergetic and polyenergetic normalized glandular dose (DgN) coefficients in mammography, Phys. Med. Biol. 62, 306 - 325 (2017). 
161

162 Biological Effectiveness of Low-Dose Mammography Quality X Rays in the Human Breast MCF-10A Cell Line, Radiat. Res. 183, 42-51 (2015).

171 F. Verhaegen and B. Reniers, Microdosimetric analysis of various mammography spectra: lineal energy distributions and ionization cluster analysis, Radiat. Res. 162, 592-599 (2004).

172 R. C. Hovey, J. F. Trott, and B. K. Vonderhaar, Establishing a framework for the functional mammary gland: from endocrinology to morphology, J. Mammary Gland Biol. Neoplasia 7, 17-38 (2002).

173 A. Tomal, I. Mazarro, E. Kakuno, and M. Poletti, Experimental determination of linear attenuation coefficient of normal, benign and malignant breast tissues, Radiat. Meas. 45, 1055-1059 (2010). 
174 N. Miksys, E. Vigneault, A.-G. Martin, L. Beaulieu, and R. M. Thomson, Largescale retrospective Monte Carlo dosimetric study for permanent implant prostate brachytherapy, Int. J. Radiat. Oncol. Biol. Phys. 97, 606-615 (2017).

175 E. D. Hay, Cell biology of extracellular matrix, Springer Science \& Business Media, New York, 2013.

176 S. Meylan, U. Vimont, S. Incerti, I. Clairand, and C. Villagrasa, Geant4-DNA simulations using complex DNA geometries generated by the DnaFabric tool, Comput. Phys. Commun. 204, 159-169 (2016).

177 J. P. Archambault and E. Mainegra-Hing, Comparison between EGSnrc, Geant4, MCNP5 and Penelope for mono-energetic electron beams, Phys. Med. Biol. 60, 4951 - 4962 (2015).

178 M. Martinov and R. M. Thomson, Heterogeneous multiscale Monte Carlo simulations for gold nanoparticle radiosensitization, Med. Phys. 44, 644-653 (2017).

179 H. Nikjoo, D. Emfietzoglou, T. Liamsuwan, R. Taleei, D. Liljequist, and S. Uehara, Radiation track, DNA damage and response - a review, Rep. Prog. Phys. 79, 116601 (2016).

180 J. E. Cygler and P. Scalchi, MOSFET dosimetry in radiotherapy, in Clinical Dosimetry Measurements in Radiotherapy, edited by D. W. O. Rogers and J. E. Cygler, Medical Physics Publishing, Madison, Wisconsin, 2009. 